\title{
Aryl Fluoride Activation Through Palladium-Magnesium Bimetallic Cooperation: A Mechanistic and Computational Study
}

Chen Wu, Samuel P. McCollom, Zhipheng Zheng, Jiadi Zhang, Sheng-chun Sha, Minyan Li, Patrick J. Walsh* and Neil C. Tomson*

Roy and Diana Vagelos Laboratories, Department of Chemistry, University of Pennsylvania, 231 South 34th Street, Philadelphia, Pennsylvania 19104-6323, United States

\section{Table of Contents}

$\begin{array}{ll}\text { 1. Experimental } & \mathrm{S} 2\end{array}$

2. Characterization of organic substrates $\quad$ S8

$\begin{array}{ll}\text { 3. NMR spectra of } \mathbf{3 a - 3} \mathbf{m} & \mathrm{S} 15\end{array}$

$\begin{array}{lr}\text { 4. NMR spectra of new phosphine ligands } & \text { S28 }\end{array}$

5. Computational details $\quad$ S35

$\begin{array}{lr}\text { 6. References } & \text { S122 }\end{array}$ 
General Methods. All reactions were performed under nitrogen using oven-dried glassware. Anhydrous CPME, 2-MeTHF, dioxane, DME, toluene and THF were purchased from Sigma-Aldrich and used without further purification. Unless otherwise stated, reagents were obtained from commercial sources and used without further purification. The progress of reactions was monitored by thin layer chromatography using Whatman Partisil K6F $250 \mu \mathrm{m}$ precoated $60 \AA$ silica gel plates and visualized by short-wavelength ultraviolet light. Silica gel (230-400 mesh, Silicycle) was used for flash chromatography. The ${ }^{1} \mathrm{H}$ NMR and ${ }^{13} \mathrm{C}\left\{{ }^{1} \mathrm{H}\right\}$ NMR spectra were obtained using a Bruker AM-500 Fourier transform NMR spectrometer at 500 and $125 \mathrm{MHz}$, respectively. Chemical shifts are reported in units of parts per million (ppm) downfield from tetramethylsilane (TMS), and all coupling constants are reported in hertz.

Ligand Synthesis Procedure. The ligands were synthesized through a modified literature protocol. ${ }^{1}$ A solution of $n$-BuLi (1.6 M in hexanes, $1.1 \mathrm{mmol}, 1.1$ equiv.) was added dropwise to a solution of bromoarenes $\left(1 \mathrm{mmol}, 1.0\right.$ equiv.) in THF $(5 \mathrm{~mL})$ at $-78{ }^{\circ} \mathrm{C}$ and the mixture was stirred for $1.5 \mathrm{~h}$ at the same temperature. A solution of chlorodialkyl phosphine or chlorodiaryl phosphine $(1.2 \mathrm{mmol}, 1.2$ equiv.) in THF $(5 \mathrm{~mL})$ was then added dropwise. The solution color quickly changed from orange to light yellow. The mixture was stirred at $-78{ }^{\circ} \mathrm{C}$ for 1 hour before it was allowed to warm to room temperature and stir overnight at room temperature. The reaction mixture was quenched with $10 \mathrm{~mL}$ saturated ammonium chloride solution, extracted with $\mathrm{Et}_{2} \mathrm{O}(3 \times 15 \mathrm{~mL})$ and dried with sodium sulfate. The sodium sulfate was filtrate off, the organic solution was 
collected and the volatile materials in the filtrate were removed under reduced pressure. The residue was loaded onto a silica gel column and purified by flash chromatography.

Pd-Catalyzed Kumada Cross-coupling of Aryl Fluorides with Phenyl Grignard Reagents. General Procedure A. An oven-dried $10 \mathrm{~mL}$ reaction vial equipped with a stir bar was placed under a nitrogen atmosphere in the dry box. A stock solution containing $\operatorname{Pd}(\mathrm{OAc})_{2}$ (11.2 mg, $0.050 \mathrm{mmol}, 5 \mathrm{~mol} \%)$ and DavePhos (39.3 mg, $0.010 \mathrm{mmol}, 10 \mathrm{~mol} \%$ ) in 10.0 $\mathrm{mL}$ dry DME was prepared in the dry box and stirred for $30 \mathrm{~min}$. Then $1 \mathrm{~mL}$ of stock solution was withdrawn by syringe and added to the $10 \mathrm{~mL}$ reaction vial. The corresponding aryl fluoride $(0.1 \mathrm{mmol}, 1$ equiv) was added to the reaction mixture followed by 4-tert-butylphenyl magnesium bromide (2.0 $\mathrm{M}$ in diethyl ether, $0.125 \mathrm{~mL}$, $0.25 \mathrm{mmol}, 2.5$ equiv) or 4-methoxyphenyl magnesium bromide (2.0 $\mathrm{M}$ in diethyl ether, $0.125 \mathrm{~mL}, 0.25 \mathrm{mmol}, 2.5$ equiv) at room temperature in the dry box and the solution turned slightly yellow. The vial was then capped, taken out of the dry box, and stirred at $50{ }^{\circ} \mathrm{C}$. After 4 hours, the capped vial was cooled to room temperature, opened to the air, quenched with three drops of $\mathrm{H}_{2} \mathrm{O}$, diluted with $3 \mathrm{~mL}$ of ethyl acetate, and filtered over a pad of silica. The pad was rinsed with an additional $10 \mathrm{~mL}$ ethyl acetate and the solution was concentrated in vacuo. The crude material was loaded onto a silica gel column and purified by flash chromatography.

High Throughput Experimentation Screens. Ligand screening of aryl fluorides with aryl Grignard reagents: Experiments were set up inside a glovebox under a nitrogen atmosphere. Two 24-well aluminum blocks containing $1 \mathrm{~mL}$ glass vials were predosed with $\mathrm{Pd}(\mathrm{OAc})_{2}(1 \mu \mathrm{mol})$ and 47 different phosphine ligands $(2 \mu \mathrm{mol}$ for monodentate 
phosphine ligands and $1 \mu \mathrm{mol}$ for bidentate ligands) in THF. The solvent was removed to dryness using a J-Kem blow down block. A parylene stir bar was then added to each reaction vial. The 4-fluorobiphenyl (10 $\mu \mathrm{mol} /$ reaction $)$ and 4-tert-butylphenyl magnesium bromide ( $25 \mu \mathrm{mol} /$ reaction) were then dosed together into each reaction vial as a solution in THF $(100 \mu \mathrm{L}, 0.1 \mathrm{M})$ at room temperature. The 24 -well plates were then sealed and stirred for $16 \mathrm{~h}$ at $80{ }^{\circ} \mathrm{C}$. Work up: The plates were cooled to room temperature. Upon opening the plate to air, $500 \mu \mathrm{L}$ of acetonitrile containing 4,4'-di-tert-butyldiphenyl (1.0 $\mu$ mmol, used as an internal standard to measure HPLC yields) was added into each vial. The plate was covered again and the vials stirred for $10 \mathrm{~min}$ to ensure good homogenization. Into a separate 96-well LC block was added $700 \mu \mathrm{L}$ of acetonitrile, followed by $40 \mu \mathrm{L}$ of each of the diluted reaction mixtures. The LC block was then sealed with a silicon-rubber storage mat and mounted on an automated HPLC instrument for analysis.
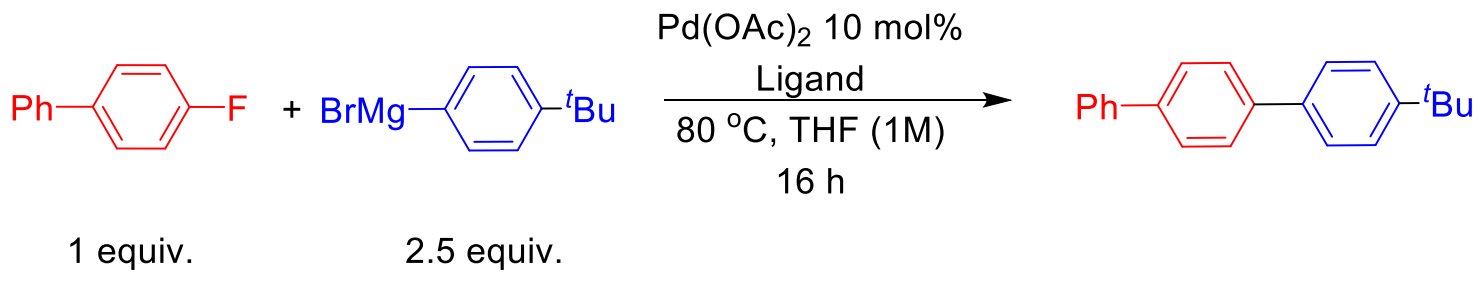

\begin{tabular}{|l|l|l|}
\hline Entry & Ligand & Prod/IS \\
\hline 1 & Benzyldi-1-adamantylphosphine (cataCXium ABn) & 13.42 \\
\hline 2 & 2-Dicyclohexylphosphino-2'-methylbiphenyl (MePhos) & 13.37 \\
\hline 3 & $\begin{array}{l}\text { N-phenyl-2-(dicyclohexylphosphino)pyrrole (cataCXium PCy) } \\
\text { 2-Dicyclohexylphosphino-2'-(N,N-dimethylamino)biphenyl } \\
\text { (DavePhos) }\end{array}$ & 12.06 \\
\hline 4 & (2-Biphenyl)dicyclohexylphosphine (Cy-JohnPhos ) & 11.76 \\
\hline 5 & & 11.45 \\
\hline
\end{tabular}




\begin{tabular}{|c|c|c|}
\hline 6 & 4,6-Bis(diphenylphosphino)phenoxazine (NIXANTPHOS) & 6.00 \\
\hline 7 & $\begin{array}{l}\text { 5-(Di-t-butylphosphino)-1', 3', 5'-triphenyl-1'H-[1,4']bipyrazole } \\
\text { (BippyPhos) }\end{array}$ & 5.88 \\
\hline 8 & $\begin{array}{l}\text { 2-(Dicyclohexylphosphino)-1-(2,4,6-trimethyl-phenyl)-1H- } \\
\text { imidazole (cataCXium PICy) }\end{array}$ & 5.16 \\
\hline 9 & 2-(Di-t-butylphosphino)-2'-methylbiphenyl (tBu-MePhos) & 5.14 \\
\hline 10 & 1,2,3,4,5-Pentaphenyl-1'-(di-t-butylphosphino)ferrocene (QPhos) & 5.09 \\
\hline 11 & $\begin{array}{l}\text { 1-[2-[Bis(t-butyl)phosphino]phenyl]-3,5-diphenyl-1H-pyrazole } \\
\text { (Trippyphos) }\end{array}$ & 5.03 \\
\hline 12 & 2-(Di-t-butylphosphino)biphenyl (JohnPhos) & 5.02 \\
\hline 13 & $\begin{array}{l}\text { Dicyclohexyl-[3,6-dimethoxy-2-(2,4,6- } \\
\text { triisopropylphenyl)phenyl]phosphane (Brettphos) }\end{array}$ & 5.02 \\
\hline 14 & Di(1-adamantyl)-2-morpholinophenylphosphine (MorDal Phos) & 4.83 \\
\hline 15 & $\begin{array}{l}\text { 2-Di-tert-butylphosphino-2',4',6'-triisopropylbiphenyl (Di-tBu- } \\
\text { XPhos) }\end{array}$ & 4.82 \\
\hline 16 & $\begin{array}{l}\text { 2-Di-tert-butylphosphino-3,4,5,6-tetramethyl-2',4',6'-triisopropyl- } \\
\text { 1,1'-biphenyl (Me4-t-Bu XPhos) }\end{array}$ & 4.77 \\
\hline 17 & $\begin{array}{l}\text { (2R)-1-[(1R)-1-[Bis(1,1-dimethylethyl)phosphino]ethyl]-2- } \\
\text { (dicyclohexylphosphino)ferrocene (Josiphos SL-J009-1) }\end{array}$ & 4.74 \\
\hline 18 & Di-t-butyl-(1-phenylindol-2-yl)phosphane (cataCXium PIntB) & 4.68 \\
\hline 19 & N-phenyl-2-(di-t-butylphosphino)pyrrole (CataXCium PtB) & 4.50 \\
\hline 20 & 9,9-Dimethyl-4,5-bis(diphenylphosphino)xanthene (Xantphos) & 4.48 \\
\hline 21 & Dicyclohexyl(4-(N,N-dimethylamino)phenyl)phosphine (A-caPhos) & 4.27 \\
\hline 22 & 2-Di-tert-butylphosphino-3-Methoxy-6-Methyl-2'-4'-6'- & 4.19 \\
\hline
\end{tabular}




\begin{tabular}{|c|c|c|}
\hline & triisopropylbiphenyl (RockPhos) & \\
\hline 23 & N,N'-dicyclohexyl-1-diphenylphosphanyl-formamidine (DCyPF) & 4.16 \\
\hline 24 & $\begin{array}{l}\text { 2-(Di-tert-butylphosphino)-2',4',6'- } \quad \text { triisopropyl-3,6- } \\
\text { dimeth+D25:E26oxy-1,1'-biphenyl (tBu-BrettPhos) }\end{array}$ & 4.09 \\
\hline 25 & N-(dicyclohexylphosphino)-2-(2'-methylphenyl)-1H-indole & 4.05 \\
\hline 26 & $\begin{array}{l}\text { 2-Dicyclohexylphosphino-2',6'-di-i-propoxy-1,1'-biphenyl } \\
\text { (RuPhos) }\end{array}$ & 4.05 \\
\hline 27 & $\begin{array}{l}\text { 2'-(Dicyclohexylphosphino)acetophenone ethylene ketal } \\
\text { (SymPhos) }\end{array}$ & 4.03 \\
\hline 28 & 1,2-Bis(diphenylphosphino)ethane & 3.93 \\
\hline 29 & Bis[(2-dicyclohexylphosphino)phenyl] ether (DCEPhos) & 3.93 \\
\hline 30 & 2-Dicyclohexylphosphino-2',6'-dimethoxy-1,1'-biphenyl (SPhos) & 3.87 \\
\hline 31 & Tri(o-tolyl)phosphine & 3.84 \\
\hline 32 & $\begin{array}{l}\text { 2-Dicyclohexylphosphino-2',6'-bis(N,N-dimethylamino)biphenyl } \\
\text { (CPhos) }\end{array}$ & 3.81 \\
\hline 33 & Rac-2-(Di-tert-butylphosphino)-1,1'-binaphthyl (TrixiePhos) & 3.78 \\
\hline 34 & Butyldi-1-adamantylphosphine (CataCXium A) & 3.65 \\
\hline 35 & N,N-Dimethyl 4-(Di(tert-butyl)phosphino)aniline (A-taphos) & 3.33 \\
\hline 36 & Di(1-adamantyl)-2-dimethylaminophenylphosphine (MeDal Phos) & 3.31 \\
\hline 37 & Tris(2,4,6-trimethylphenyl)phosphine ( $\left.\mathrm{PX}_{3}\right)$ & 2.31 \\
\hline 38 & 1,1'-Bis(diisopropylphosphino)ferrocene (dippf) & 2.13 \\
\hline 39 & Tricyclohexylphosphine tetrafluoroborate $\left(\mathrm{PCy}_{3} \mathrm{HBF}_{4}\right)$ & 1.99 \\
\hline 40 & Tri(furan-2-yl)phosphine & 1.79 \\
\hline
\end{tabular}




\begin{tabular}{|l|l|l|}
\hline 41 & Triphenylphospine & 1.77 \\
\hline 42 & $1,1^{\prime}-$ Bis(di-t-butylphosphino)ferrocene (dtbpf) & 1.29 \\
\hline 43 & Tri-tert-butylphosphonium tetrafluoroborate $\left(\mathrm{PtBu}_{3} \mathrm{HBF}_{4}\right)$ & 0.54 \\
\hline 44 & Di-tert-butyl(neopentyl)phosphine HBF4 & 0.54 \\
\hline 45 & {$\left[1,1^{\prime}-\right.$ Binaphthalene]-2,2'-diylbis[diphenylphosphine] (Binap) } & 0.45 \\
\hline 46 & $\begin{array}{l}\text { 2-(Dicyclohexylphosphino)-1-(2,4,6-trimethyl-phenyl)-1H- } \\
\text { imidazole (cataCXium PICy) }\end{array}$ & 0.13 \\
\hline 47 & \begin{tabular}{l}
$1,3-$-Bis(diphenylphosphino)propane (dppp) \\
\hline
\end{tabular} & 0.13 \\
\hline
\end{tabular}




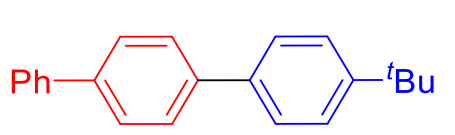

4-(tert-butyl)-1,1':4',1'"-terphenyl 3a. The reaction was performed following the General Procedure A with 4fluorobiphenyl (1a, $17.8 \mathrm{mg}, 0.1 \mathrm{mmol}$ ) and 4-tert-butylphenyl magnesium bromide $(0.125 \mathrm{~mL}, 0.25 \mathrm{mmol})$. The crude product was purified by flash chromatography on silica gel (eluted with hexanes) to give the product $(28.5 \mathrm{mg}, 99 \%)$ as a white solid. The spectroscopic data match the previously reported data. ${ }^{2}$

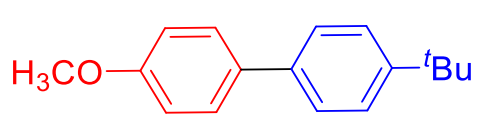

4-(tert-butyl)-4'-methoxy-1,1'-biphenyl $3 \mathrm{~b}$. The reaction was performed following the General Procedure A with 4fluoroanisole (1b, $12.6 \mathrm{mg}, 0.1 \mathrm{mmol})$ and 4-tert-butylphenyl magnesium bromide $(0.125$ $\mathrm{mL}, 0.25 \mathrm{mmol})$. The crude product was purified by flash chromatography on silica gel (eluted with hexanes) to give the product $(22.8 \mathrm{mg}, 95 \%)$ as a white solid. The spectroscopic data match the previously reported data. ${ }^{3}$

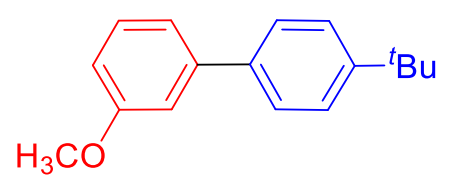

4'-(tert-butyl)-3-methoxy-1,1'-biphenyl 3c. The reaction was performed following the General Procedure A with 3fluoroanisole (1c, $12.6 \mathrm{mg}, 0.1 \mathrm{mmol})$ and 4-tert-butylphenyl magnesium bromide $(0.125$ $\mathrm{mL}, 0.25 \mathrm{mmol})$. The crude product was purified by flash chromatography on silica gel (eluted with hexanes) to give the product $(23.7 \mathrm{mg}, 99 \%)$ as a white solid. The spectroscopic data match the previously reported data. ${ }^{4}$

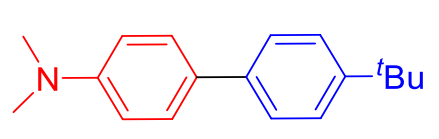

4'-(tert-butyl)-N,N-dimethyl-[1,1'-biphenyl]-4-amine 3d. The reaction was performed following the General Procedure A with 4-fluoroaniline (1d, $13.9 \mathrm{mg}, 0.1 \mathrm{mmol})$ and 4-tert-butylphenyl magnesium bromide 
$(0.125 \mathrm{~mL}, 0.25 \mathrm{mmol})$. The crude product was purified by flash chromatography on silica gel (eluted with hexanes to ethyl acetate $=50: 1)$ to give the product $(23.0 \mathrm{mg}, 91 \%)$ as a white solid. The spectroscopic data match the previously reported data. ${ }^{5}$

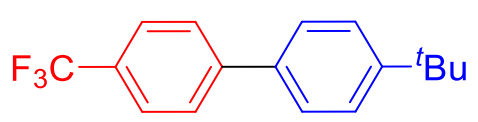

4-(tert-butyl)-4'-(trifluoromethyl)-1,1'-biphenyl 3e. The reaction was performed following the General Procedure A with 1-fluoro-4-(trifluoromethyl)benzene (1e, $16.4 \mathrm{mg}, 0.1 \mathrm{mmol})$ and 4-tert-butylphenyl magnesium bromide $(0.125 \mathrm{~mL}, 0.25 \mathrm{mmol})$. The crude product was purified by flash chromatography on silica gel (eluted with hexanes) to give the product $(21.6 \mathrm{mg}, 78 \%)$ as a white solid. The spectroscopic data match the previously reported data. ${ }^{6}$

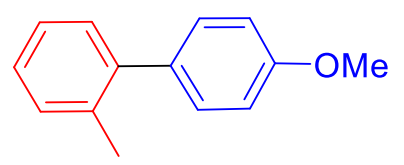

4'-methoxy-2-methyl-1,1'-biphenyl 3f. The reaction was
performed following the General Procedure A with 2fluorotoluene (1f, $11.0 \mathrm{mg}, 0.1 \mathrm{mmol})$ and 4-methoxy-phenyl magnesium bromide $(0.125$ $\mathrm{mL}, 0.25 \mathrm{mmol})$. The crude product was purified by flash chromatography on silica gel (eluted with hexanes) to give the product $(14.4 \mathrm{mg}, 73 \%)$ as a white solid. The spectroscopic data match the previously reported data. ${ }^{7}$

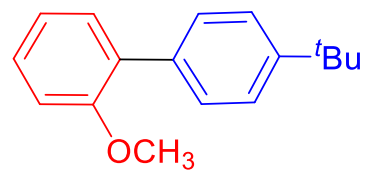

4'-(tert-butyl)-2-methoxy-1,1'-biphenyl 3g. The reaction was performed following the General Procedure A with 2-fluoroanisole $(1 \mathrm{~g}, 12.6 \mathrm{mg}, 0.1 \mathrm{mmol})$ and 4-tert-butylphenyl magnesium bromide $(0.125 \mathrm{~mL}, 0.25$ mmol). The crude product was purified by flash chromatography on silica gel (eluted with hexanes) to give the product (18.9 $\mathrm{mg}, 79 \%)$ as a white solid. The spectroscopic data match the previously reported data. ${ }^{8}$ 


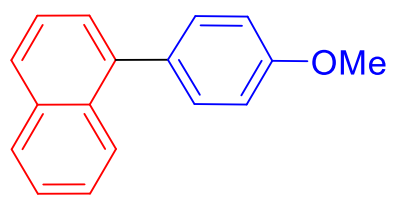

1-(4'-methoxy-[1,1'-biphenyl]-4-yl)naphthalene $3 \mathrm{~h} . \quad$ The reaction was performed following the General Procedure A with 1-fluoronaphthalene (1h, $14.6 \mathrm{mg}, 0.1 \mathrm{mmol})$ and 4-methoxyphenyl magnesium bromide $(0.125 \mathrm{~mL}, 0.25 \mathrm{mmol})$. The crude product was purified by flash chromatography on silica gel (eluted with hexanes) to give the product ( $22.4 \mathrm{mg}$, $96 \%)$ as a white solid. The spectroscopic data match the previously reported data. ${ }^{9}$

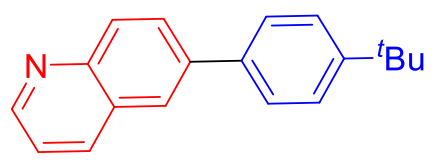

6-(4-(tert-butyl)phenyl)quinoline $3 \mathbf{3}$. The reaction was performed following the General Procedure A with 6fluoroquinoline (1i, $14.7 \mathrm{mg}, 0.1 \mathrm{mmol})$ and 4-tert-butylphenyl magnesium bromide $(0.125 \mathrm{~mL}, 0.25 \mathrm{mmol})$. The crude product was purified by flash chromatography on silica gel (eluted with hexanes to ethyl acetate $\left.=10: 1, \mathrm{R}_{\mathrm{f}}=0.16\right)$ to give the product $(15.4$ $\mathrm{mg}, 59 \%)$ as a white solid. M.P. $=94.5-95.5{ }^{\circ} \mathrm{C} .{ }^{1} \mathrm{H} \mathrm{NMR}\left(500 \mathrm{MHz}, \mathrm{CDCl}_{3}\right) \delta 8.90(\mathrm{dd}$, $J=4.2,1.6 \mathrm{~Hz}, 1 \mathrm{H}), 8.25-8.12(\mathrm{~m}, 2 \mathrm{H}), 8.02-7.96(\mathrm{~m}, 2 \mathrm{H}), 7.67(\mathrm{~d}, J=8.4 \mathrm{~Hz}, 2 \mathrm{H})$, $7.53(\mathrm{~d}, J=8.4 \mathrm{~Hz}, 2 \mathrm{H}), 7.42(\mathrm{dd}, J=8.3,4.2 \mathrm{~Hz}, 1 \mathrm{H}), 1.39(\mathrm{~s}, 9 \mathrm{H}) \mathrm{ppm} .{ }^{13} \mathrm{C}$ NMR $(126$ $\left.\mathrm{MHz}, \mathrm{CDCl}_{3}\right) \delta 151.63,146.95,140.88,139.83,136.48,131.44,129.39,128.90,127.22$, 126.18, 125.22, 125.04, 121.33, 34.71, 31.34. IR (thin film) 2960, 2875, 1493, 1402, 1297, $826 \mathrm{~cm}^{-1}$. HRMS (EI) m/z: [M]+ calcd for $\mathrm{C}_{19} \mathrm{H}_{19} \mathrm{~N}$ 261.1517; Found 261.1488.

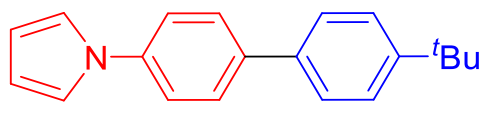

1-(4'-(tert-butyl)-[1,1'-biphenyl]-4-yl)-1H-pyrrole $3 \mathbf{j}$. The reaction was performed following the General Procedure A with 1-(4-fluorophenyl)-1H-pyrrole (1j, $16.1 \mathrm{mg}, 0.1 \mathrm{mmol}$ ) and 4-tert-butylphenyl magnesium bromide $(0.125 \mathrm{~mL}, 0.25 \mathrm{mmol})$. The crude product was purified by flash 
chromatography on silica gel (eluted with hexanes, $\mathrm{R}_{\mathrm{f}}=0.15$ ) to give the product $(25.1 \mathrm{mg}$, 91\%) as a white solid. M.P. $=193-195{ }^{\circ} \mathrm{C} .{ }^{1} \mathrm{H}$ NMR $\left(500 \mathrm{MHz}, \mathrm{CDCl}_{3}\right) \delta 7.64(\mathrm{~d}, J=8.5$ $\mathrm{Hz}, 2 \mathrm{H}), 7.54(\mathrm{~d}, J=8.4 \mathrm{~Hz}, 2 \mathrm{H}), 7.46(\mathrm{dd}, J=15.1,8.5 \mathrm{~Hz}, 4 \mathrm{H}), 7.12(\mathrm{t}, J=2.1 \mathrm{~Hz}$, 2H), $6.36(\mathrm{t}, J=2.1 \mathrm{~Hz}, 2 \mathrm{H}), 1.37(\mathrm{~s}, 9 \mathrm{H}) \mathrm{ppm} .{ }^{13} \mathrm{C} \mathrm{NMR}\left(125 \mathrm{MHz}, \mathrm{CDCl}_{3}\right)$ 150.64, $139.89,138.58,137.45,128.17,126.73,126.01,120.88,119.46,110.62,34.73,31.54$ ppm. IR (thin film) 2961, 1506, 1396, 1330, 1250, 1076, 818, 723, $669 \mathrm{~cm}^{-1}$. HRMS (EI) $\mathrm{m} / \mathrm{z}:[\mathrm{M}]+$ calcd for $\mathrm{C}_{20} \mathrm{H}_{21} \mathrm{~N}$ 275.1674; Found 275.1691.

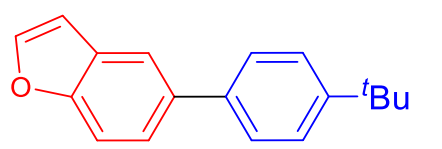

5-(4-(tert-butyl)phenyl)benzofuran 3k. The reaction was performed following the General Procedure A with 5fluorobenzofuran $(\mathbf{1 k}, 13.6 \mathrm{mg}, 0.1 \mathrm{mmol})$ and 4-tert-butylphenyl magnesium bromide $(0.125 \mathrm{~mL}, 0.25 \mathrm{mmol})$. The crude product was purified by flash chromatography on silica gel (eluted with hexanes, $\mathrm{R}_{\mathrm{f}}=0.35$ ) to give the product (13.0 $\mathrm{mg}, 50 \%$ ) as a white solid. M.P. $=96-97{ }^{\circ} \mathrm{C} .{ }^{1} \mathrm{H}$ NMR $\left(500 \mathrm{MHz}, \mathrm{CDCl}_{3}\right) \delta 7.78(\mathrm{~d}, J=1.3 \mathrm{~Hz}, 1 \mathrm{H}), 7.64(\mathrm{~d}, J$ $=2.2 \mathrm{~Hz}, 1 \mathrm{H}), 7.58-7.50(\mathrm{~m}, 4 \mathrm{H}), 7.47(\mathrm{~d}, J=8.4 \mathrm{~Hz}, 2 \mathrm{H}), 6.80(\mathrm{~d}, J=2.0 \mathrm{~Hz}, 1 \mathrm{H})$, 1.37 (s, 9H) ppm. ${ }^{13} \mathrm{C}$ NMR $\left(125 \mathrm{MHz}, \mathrm{CDCl}_{3}\right) \delta 154.66,150.05,145.67,138.95,136.53$, $128.11,127.28,125.90,124.11,119.70,111.63,107.00,34.71,31.61 \mathrm{ppm}$. IR (thin film) 2960, 1460, 1131, 1030, 814, 766, $740 \mathrm{~cm}^{-1}$. HRMS (EI) m/z: [M]+ calcd for $\mathrm{C}_{18} \mathrm{H}_{18} \mathrm{O}$ 250.1538; Found 250.1529.

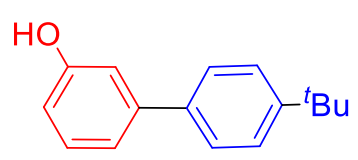

4'-(tert-butyl)-[1,1'-biphenyl]-3-ol 31. The reaction was performed following the General Procedure A with 3-fluorophenol (11, 11.2 $\mathrm{mg}, 0.1 \mathrm{mmol})$ and 4-tert-butylphenyl magnesium bromide $(0.125 \mathrm{~mL}, 0.25 \mathrm{mmol})$. The crude product was purified by flash chromatography on silica gel (eluted with hexanes to 
ethyl acetate $=10: 1)$ to give the product $(10.0 \mathrm{mg}, 44 \%)$ as a white solid. The spectroscopic data match the previously reported data. ${ }^{10}$

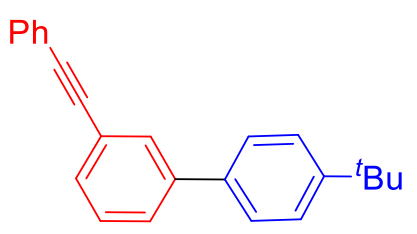

4'-(tert-butyl)-3-(phenylethynyl)-1,1'-biphenyl 3m. The reaction was performed following the General Procedure A with (3-fluorophenyl)phenylacetylene (1 m, $19.6 \mathrm{mg}, 0.1 \mathrm{mmol})$ and 4-tert-butylphenyl magnesium bromide $(0.125 \mathrm{~mL}, 0.25 \mathrm{mmol})$. The crude product was purified by flash chromatography on silica gel (eluted with hexanes, $R_{f}=0.30$ ) to give the product $(17.0 \mathrm{mg}, 55 \%)$ as a white solid. M.P. $=73-75{ }^{\circ} \mathrm{C} .{ }^{1} \mathrm{H}$ NMR $\left(500 \mathrm{MHz}, \mathrm{CDCl}_{3}\right) \delta$ $7.76(\mathrm{~d}, J=1.5 \mathrm{~Hz}, 1 \mathrm{H}), 7.57-7.52(\mathrm{~m}, 5 \mathrm{H}), 7.50-7.44(\mathrm{~m}, 3 \mathrm{H}), 7.40(\mathrm{t}, J=7.7 \mathrm{~Hz}$, 1H), $7.35(\mathrm{dd}, J=4.9,2.1 \mathrm{~Hz}, 3 \mathrm{H}), 1.36(\mathrm{~s}, 9 \mathrm{H}) \mathrm{ppm} .{ }^{13} \mathrm{C}\left\{{ }^{1} \mathrm{H}\right\}$ NMR $(125 \mathrm{MHz}, \mathrm{CDCl} 3)$ $\delta 150.69,141.26,137.44,131.67,130.20,130.12,128.76,128.38,128.30,126.99$, $126.76,125.80,123.66,123.30,89.49,89.40,34.58,31.37 \mathrm{ppm}$. IR (thin film) 2962 , 1600, 1491, 1392, 1269, 836, 792, 754, $691 \mathrm{~cm}^{-1}$. HRMS (EI) m/z: [M]+ calcd for $\mathrm{C}_{24} \mathrm{H}_{22}$ 310.1722; Found 310.1722.

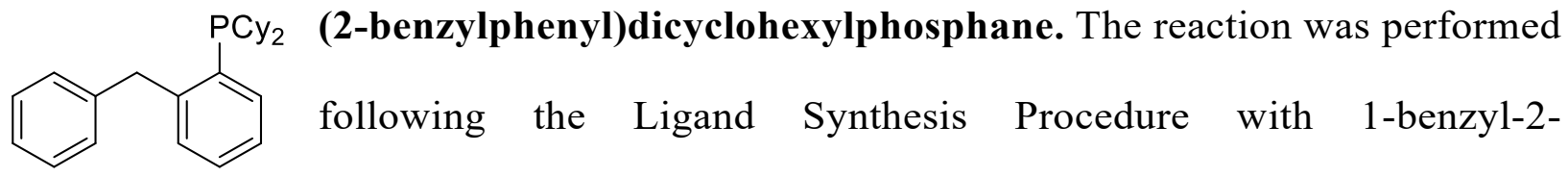
bromobenzene (247 mg, $1 \mathrm{mmol})$ and chlorodicyclohexylphosphine (280 mg, $1.2 \mathrm{mmol})$. The crude product was purified by flash chromatography on silica gel (eluted with hexanes) to give the product (145.6 mg, 40\%) as a colorless oil. ${ }^{1} \mathrm{H}$ NMR (360 MHz, $\left.\mathrm{CDCl}_{3}\right) \delta 7.51(\mathrm{~d}, J=7.1 \mathrm{~Hz}, 1 \mathrm{H}), 7.34-7.24(\mathrm{~m}, 4 \mathrm{H}), 7.19(\mathrm{~d}, J=7.3 \mathrm{~Hz}, 4 \mathrm{H}), 4.47(\mathrm{~s}$, 2H), $1.90(\mathrm{dd}, J=16.4,7.5 \mathrm{~Hz}, 4 \mathrm{H}), 1.85-1.51(\mathrm{~m}, 8 \mathrm{H}), 1.37-1.13(\mathrm{~m}, 8 \mathrm{H}), 1.11-$ $0.94(\mathrm{~m}, 2 \mathrm{H}) .{ }^{13} \mathrm{C}\left\{{ }^{1} \mathrm{H}\right\} \operatorname{NMR}\left(91 \mathrm{MHz}, \mathrm{CDCl}_{3}\right) \delta 190.71,147.89\left(\mathrm{~d}, J_{C-P}=26.5 \mathrm{~Hz}\right)$, 
141.87, $134.27\left(\mathrm{~d}, J_{C-P}=18.6 \mathrm{~Hz}\right), 132.83\left(\mathrm{~d}, J_{C-P}=3.3 \mathrm{~Hz}\right), 130.34\left(\mathrm{~d}, J_{C-P}=5.3 \mathrm{~Hz}\right)$, $129.18,128.58,128.17,125.61\left(\mathrm{~d}, J_{C-P}=13.9 \mathrm{~Hz}\right), 40.15\left(\mathrm{~d}, J_{C-P}=25.2 \mathrm{~Hz}\right), 34.12\left(\mathrm{~d}, J_{C-}\right.$ $\left.{ }_{P}=12.6 \mathrm{~Hz}\right), 30.43\left(\mathrm{~d}, J_{C-P}=16.6 \mathrm{~Hz}\right), 29.21\left(\mathrm{~d}, J_{C-P}=9.3 \mathrm{~Hz}\right), 27.20\left(\mathrm{~d}, J_{C-P}=20.9 \mathrm{~Hz}\right)$, 27.18, 26.42.. ${ }^{31} \mathrm{P}\left\{{ }^{1} \mathrm{H}\right\}$ NMR (146 MHz, $\left.\mathrm{CDCl}_{3}\right) \delta-14.89 \mathrm{ppm}$. IR (thin film) 3058, 3025, 2923, 2849, 2360, 2341, 1602, 1589, 1447, 1176, 1122, $749 \mathrm{~cm}^{-1}$. HRMS (ESI) m/z: $[\mathrm{M}+\mathrm{H}]+$ calcd for $\mathrm{C}_{25} \mathrm{H}_{34} \mathrm{P} 365.2398$; Found 365.2413.

following the Ligand Synthesis Procedure with 1-benzyl-2bromobenzene (247 mg, $1 \mathrm{mmol}$ ) and chlorodiphenylphosphine (264 mg, $1.2 \mathrm{mmol})$. The crude product was purified by flash chromatography on silica gel (eluted with hexanes) to give the product $(116.2 \mathrm{mg}, 33 \%)$ as a colorless oil. ${ }^{1} \mathrm{H}$ NMR $\left(500 \mathrm{MHz}, \mathrm{CDCl}_{3}\right) \delta 7.34-$ $7.28(\mathrm{~m}, 6 \mathrm{H}), 7.26-7.20(\mathrm{~m}, 5 \mathrm{H}), 7.17$ (t, $J=7.3 \mathrm{~Hz}, 2 \mathrm{H}), 7.10(\mathrm{dd}, J=17.4,7.8 \mathrm{~Hz}$, 5H), $6.92-6.87(\mathrm{~m}, 1 \mathrm{H}), 4.23(\mathrm{~s}, 2 \mathrm{H}) \mathrm{ppm} .{ }^{13} \mathrm{C}\left\{{ }^{1} \mathrm{H}\right\} \mathrm{NMR}\left(125 \mathrm{MHz}, \mathrm{CDCl}_{3}\right) \delta 145.69$ $\left(\mathrm{d}, J_{C-P}=25.7 \mathrm{~Hz}\right), 140.65,136.73\left(\mathrm{~d}, J_{C-P}=10.5 \mathrm{~Hz}\right), 136.01\left(\mathrm{~d}, J_{C-P}=12.8 \mathrm{~Hz}\right), 133.98$ $\left(\mathrm{d}, J_{C-P}=19.7 \mathrm{~Hz}\right), 133.67,129.93\left(\mathrm{~d}, J_{C-P}=5.0 \mathrm{~Hz}\right), 129.38,129.04,128.68,128.54(\mathrm{~d}$, $\left.J_{C-P}=7.3 \mathrm{~Hz}\right), 128.29,126.50,125.96,40.01\left(\mathrm{~d}, J_{C-P}=22.5 \mathrm{~Hz}\right) \mathrm{ppm} .{ }^{31} \mathrm{P}\left\{{ }^{1} \mathrm{H}\right\} \mathrm{NMR}(270$ $\mathrm{MHz}, \mathrm{CDCl}_{3}$ ) $\delta-14.47 \mathrm{ppm}$. IR (thin film) 3054, 3028, 2980, 2956, 2855, 1740, 1589, 1447, 1239, $705 \mathrm{~cm}^{-1}$. HRMS (ESI) m/z: $[\mathrm{M}+\mathrm{H}]+$ calcd for $\mathrm{C}_{25} \mathrm{H}_{22} \mathrm{P}$ 353.1459; Found 353.1447 .

$\mathrm{PCy}_{2}$ dicyclohexyl(o-tolyl)phosphane. The reaction was performed following the
Ligand Synthesis Procedure with 2-bromotoluene $(170 \mathrm{mg}, 1 \mathrm{mmol})$ and chlorodicyclohexylphosphine $(280 \mathrm{mg}, 1.2 \mathrm{mmol})$. The crude product was purified by 
flash chromatography on silica gel (eluted with hexanes) to give the product (138.3 $\mathrm{mg}$, $48 \%$ ) as a white solid. The spectroscopic data match the previously reported data. ${ }^{11}$

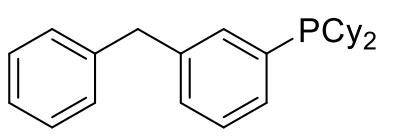

(3-benzylphenyl)dicyclohexylphosphane. The reaction was performed following the Ligand Synthesis Procedure with 1benzyl-3-bromobenzene $(247 \mathrm{mg}, 1 \mathrm{mmol})$ and chlorodicyclohexylphosphine (280 $\mathrm{mg}$, $1.2 \mathrm{mmol}$ ). The crude product was purified by flash chromatography on silica gel (eluted with hexanes) to give the product (138.5 mg, 38\%) as a colorless oil. ${ }^{1} \mathrm{H}$ NMR (500 MHz, $\left.\mathrm{CDCl}_{3}\right) \delta 7.32-7.22(\mathrm{~m}, 5 \mathrm{H}), 7.21-7.11(\mathrm{~m}, 4 \mathrm{H}), 3.99(\mathrm{~s}, 2 \mathrm{H}), 1.97-1.79(\mathrm{~m}, 4 \mathrm{H})$, $1.79-1.72(\mathrm{~m}, 2 \mathrm{H}), 1.65(\mathrm{tdd}, \mathrm{J}=9.0,6.1,3.5 \mathrm{~Hz}, 4 \mathrm{H}), 1.59-1.53(\mathrm{~m}, 2 \mathrm{H}), 1.37-1.05$ $(\mathrm{m}, 8 \mathrm{H}), 0.99-0.85(\mathrm{~m}, 2 \mathrm{H}) .{ }^{13} \mathrm{C}\left\{{ }^{1} \mathrm{H}\right\} \mathrm{NMR}\left(125 \mathrm{MHz}, \mathrm{CDCl}_{3}\right) \delta 141.13,140.43\left(\mathrm{~d}, J_{C-P}\right.$ $=7.3 \mathrm{~Hz}), 135.39\left(\mathrm{~d}, J_{C-P}=19.9 \mathrm{~Hz}\right), 134.67\left(\mathrm{~d}, J_{C-P}=17.2 \mathrm{~Hz}\right), 132.33\left(\mathrm{~d}, J_{C-P}=18.2\right.$ $\mathrm{Hz}), 129.33,128.88,128.42,127.86\left(\mathrm{~d}, J_{C-P}=7.3 \mathrm{~Hz}\right), 126.04,41.88,32.46\left(\mathrm{~d}, J_{C-P}=\right.$ $11.6 \mathrm{~Hz}), 30.02\left(\mathrm{~d}, J_{C-P}=16.3 \mathrm{~Hz}\right), 28.81\left(\mathrm{~d}, J_{C-P}=7.3 \mathrm{~Hz}\right), 27.24\left(\mathrm{~d}, J_{C-P}=12.6 \mathrm{~Hz}\right)$, $27.00\left(\mathrm{~d}, J_{C-P}=7.3 \mathrm{~Hz}\right), 26.43 \mathrm{ppm} .{ }^{31} \mathrm{P}\left\{{ }^{1} \mathrm{H}\right\} \mathrm{NMR}\left(270 \mathrm{MHz}, \mathrm{CDCl}_{3}\right) \delta 2.99 \mathrm{ppm} . \mathrm{IR}$ (thin film) 3026, 2922, 2848, 2380, 2340, 1738, 1585, 1433, 1240, 1070, 1046, 743, 696 $\mathrm{cm}^{-1}$. HRMS (ESI) m/z: [M+H]+ calcd for $\mathrm{C}_{25} \mathrm{H}_{34} \mathrm{P}$ 365.2398; Found 365.2392. 


\section{${ }^{1} \mathrm{H}$ and ${ }^{13} \mathrm{C}\left\{{ }^{1} \mathrm{H}\right\}$ NMR Spectra of compound 3a}
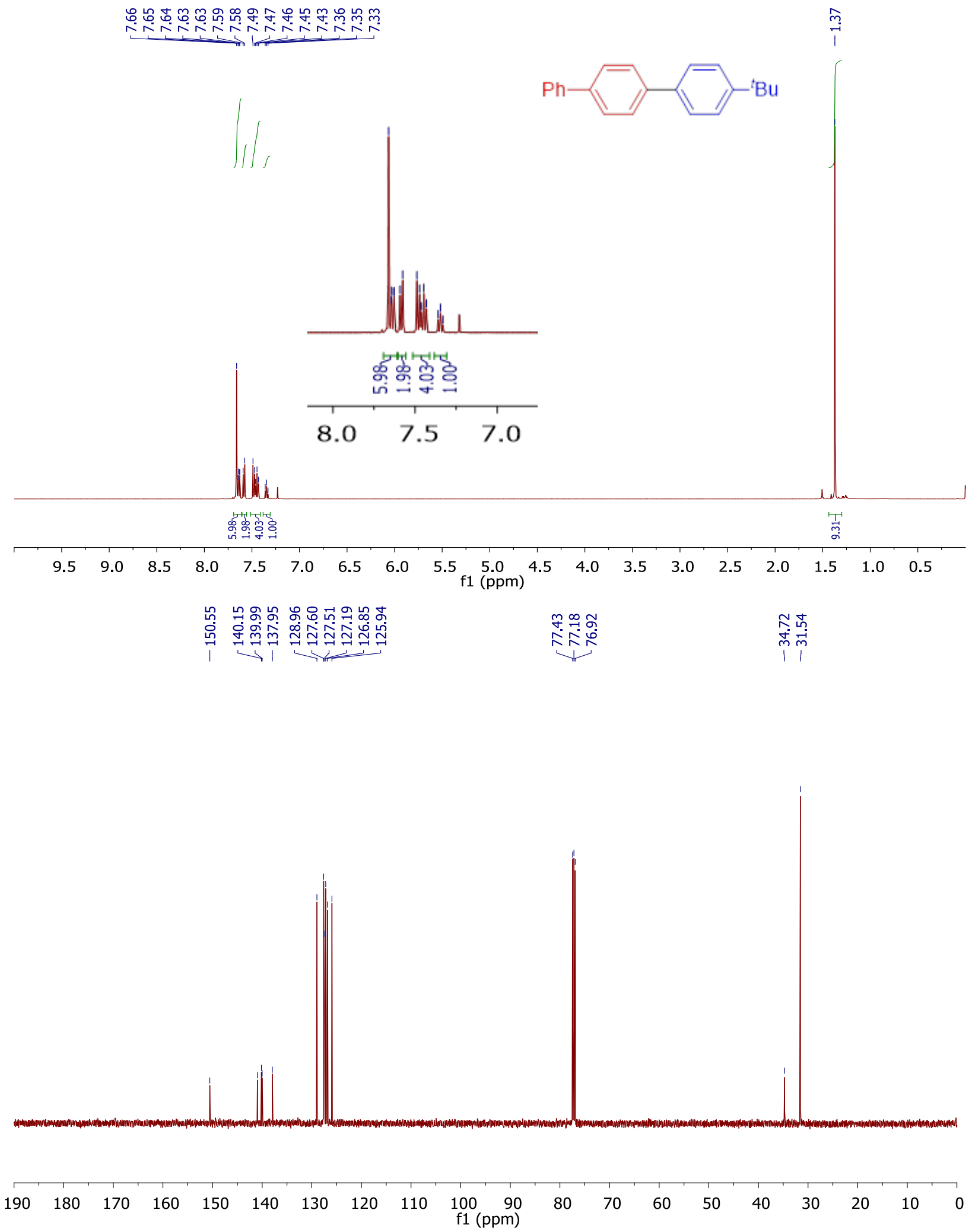


\section{${ }^{1} \mathrm{H}$ and ${ }^{13} \mathrm{C}\left\{{ }^{1} \mathrm{H}\right\}$ NMR Spectra of compound $3 b$}

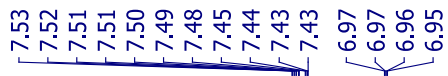

$\infty \infty \infty \infty \infty \infty$

imim
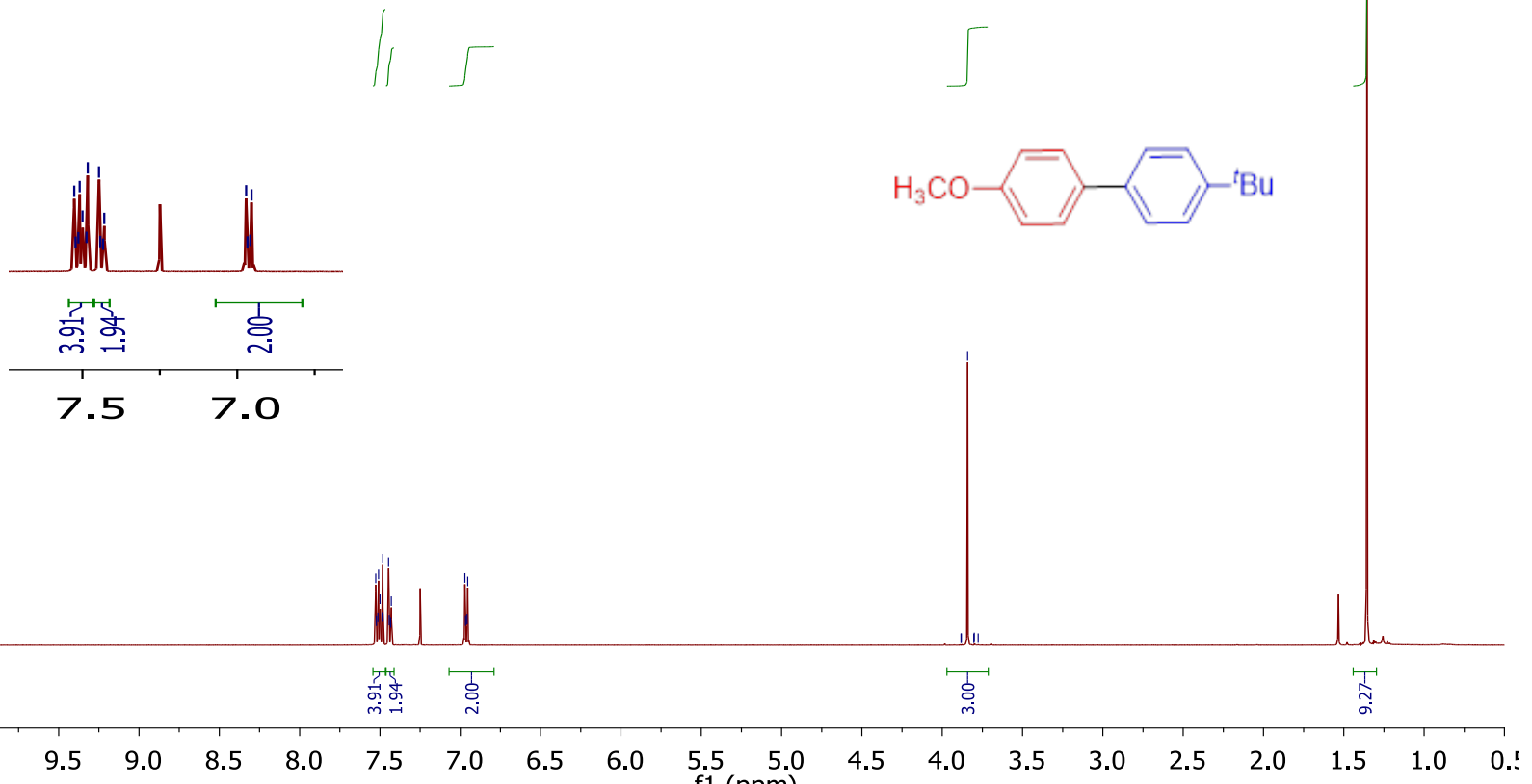

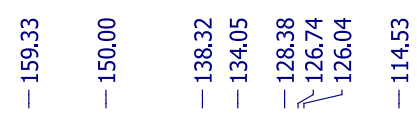

5.5
f1 (ppm)

$4.5 \quad 4.0$ .

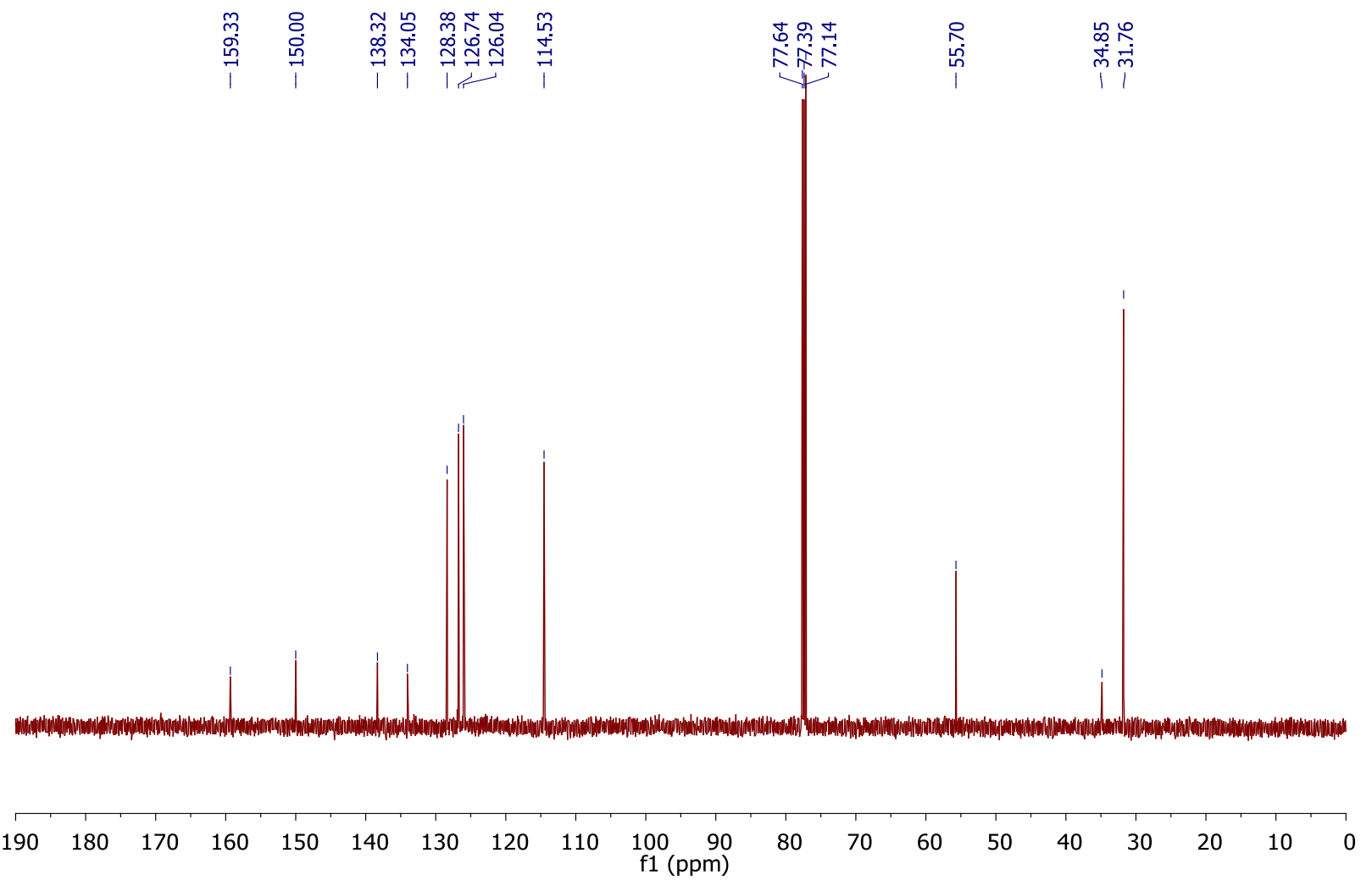




\section{${ }^{1} \mathrm{H}$ and ${ }^{13} \mathrm{C}\left\{{ }^{1} \mathrm{H}\right\}$ NMR Spectra of compound $3 \mathrm{c}$}

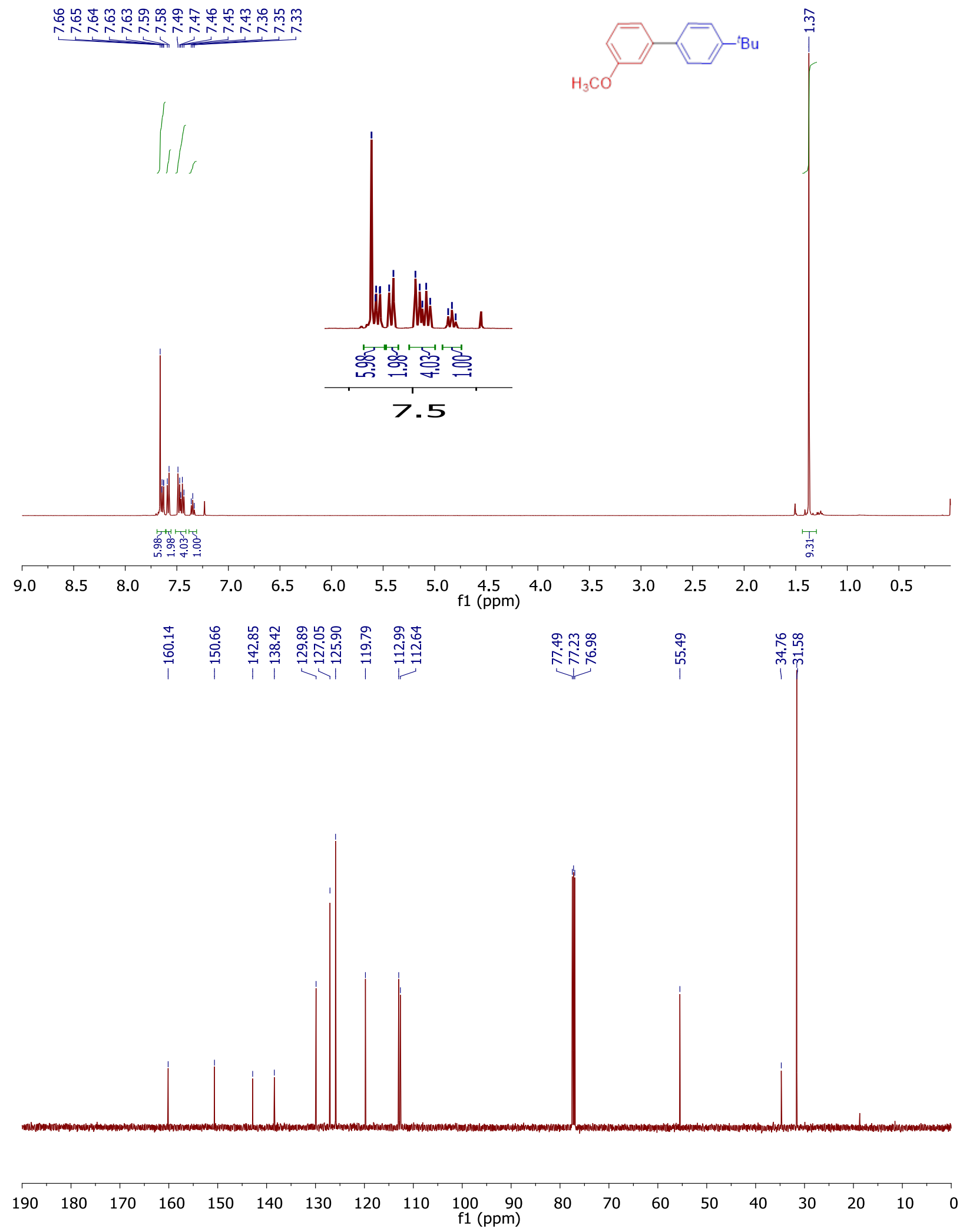




\section{${ }^{1} \mathrm{H}$ and ${ }^{13} \mathrm{C}\left\{{ }^{1} \mathrm{H}\right\}$ NMR Spectra of compound 3d}

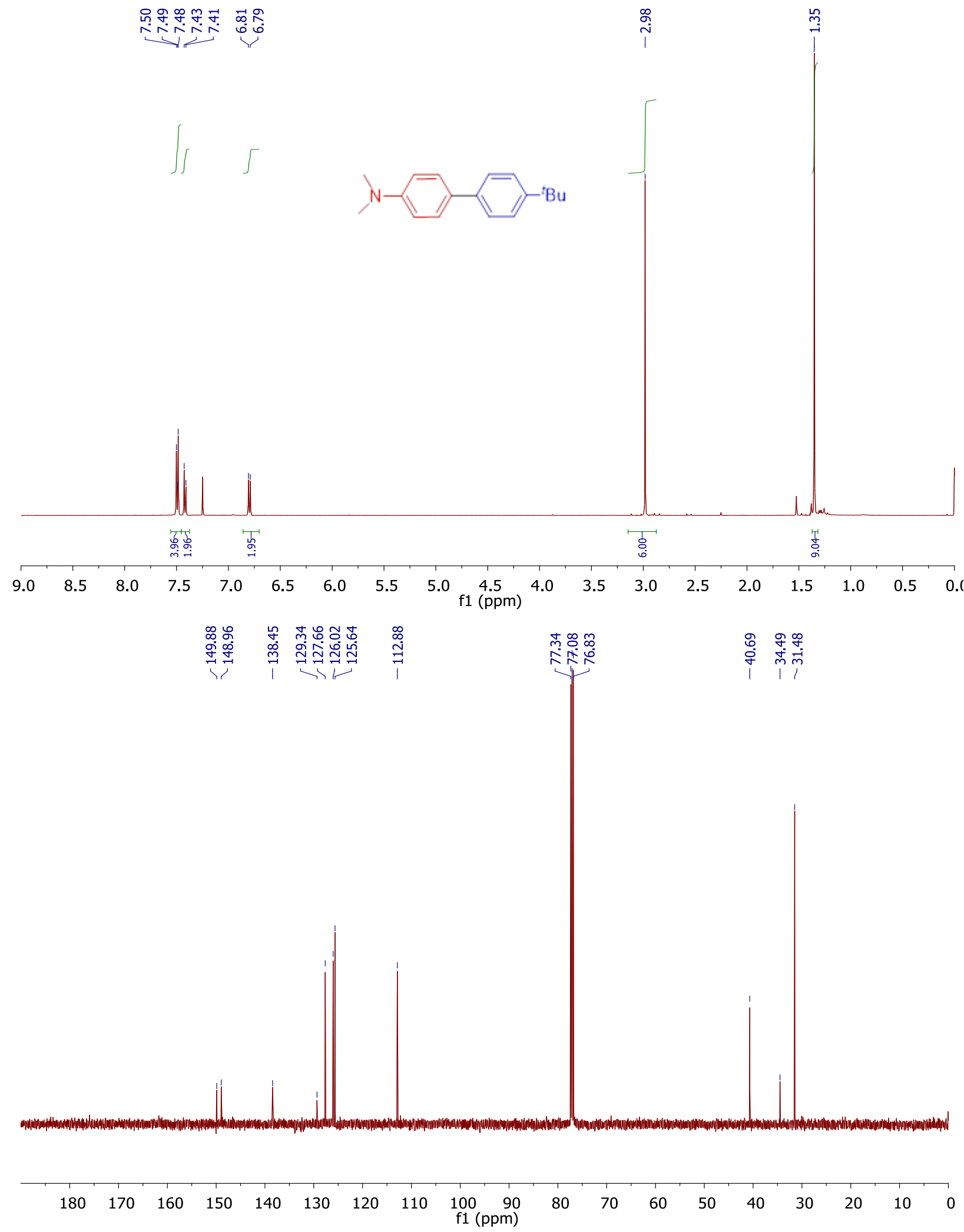




\section{${ }^{1} \mathrm{H}$ and ${ }^{13} \mathrm{C}\left\{{ }^{1} \mathrm{H}\right\}$ NMR Spectra of compound $3 e$}

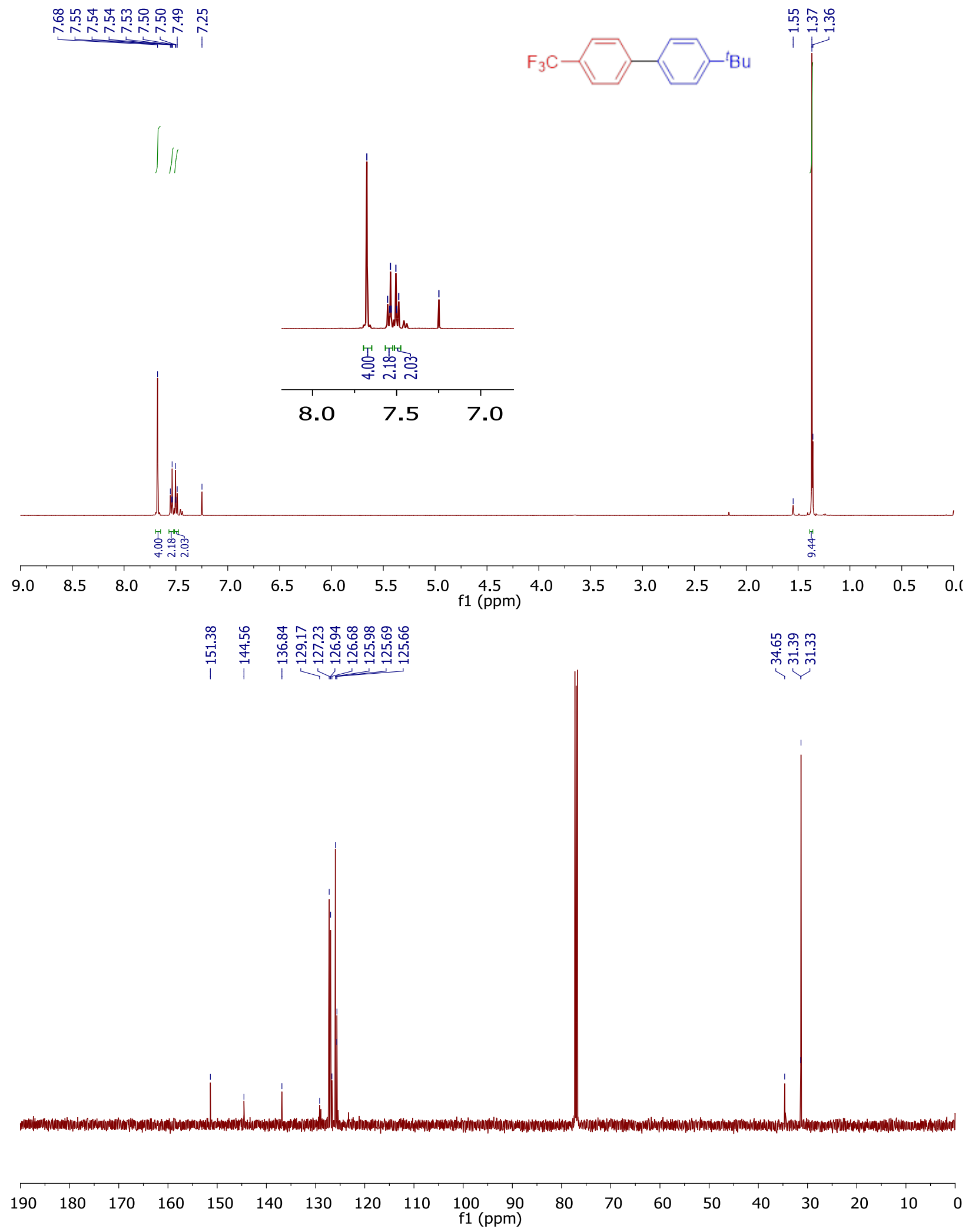




\section{${ }^{1} \mathrm{H}$ and ${ }^{13} \mathrm{C}\left\{{ }^{1} \mathrm{H}\right\}$ NMR Spectra of compound $3 f$}
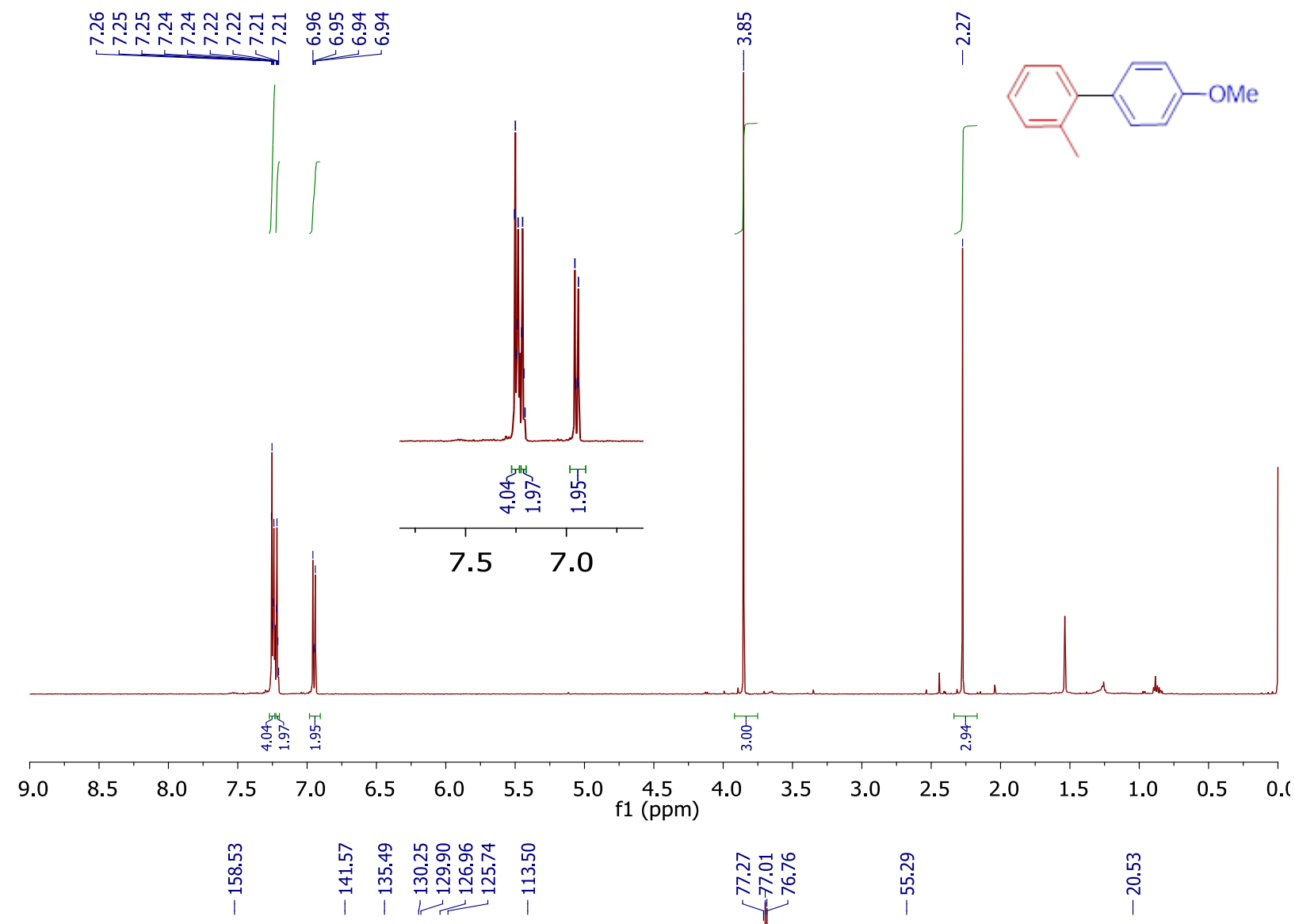

ูำ

กำ

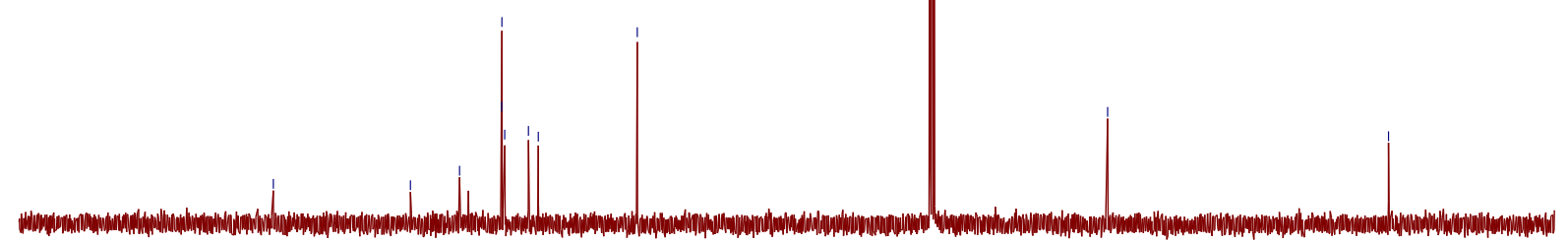

190

180

$170 \quad 160$

150

130

120

10090

$80 \quad 70$

1 


\section{${ }^{1} \mathrm{H}$ and ${ }^{13} \mathrm{C}\left\{{ }^{1} \mathrm{H}\right\}$ NMR Spectra of compound $3 \mathrm{~g}$}

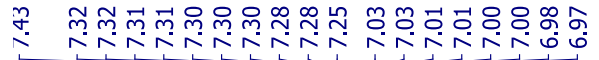

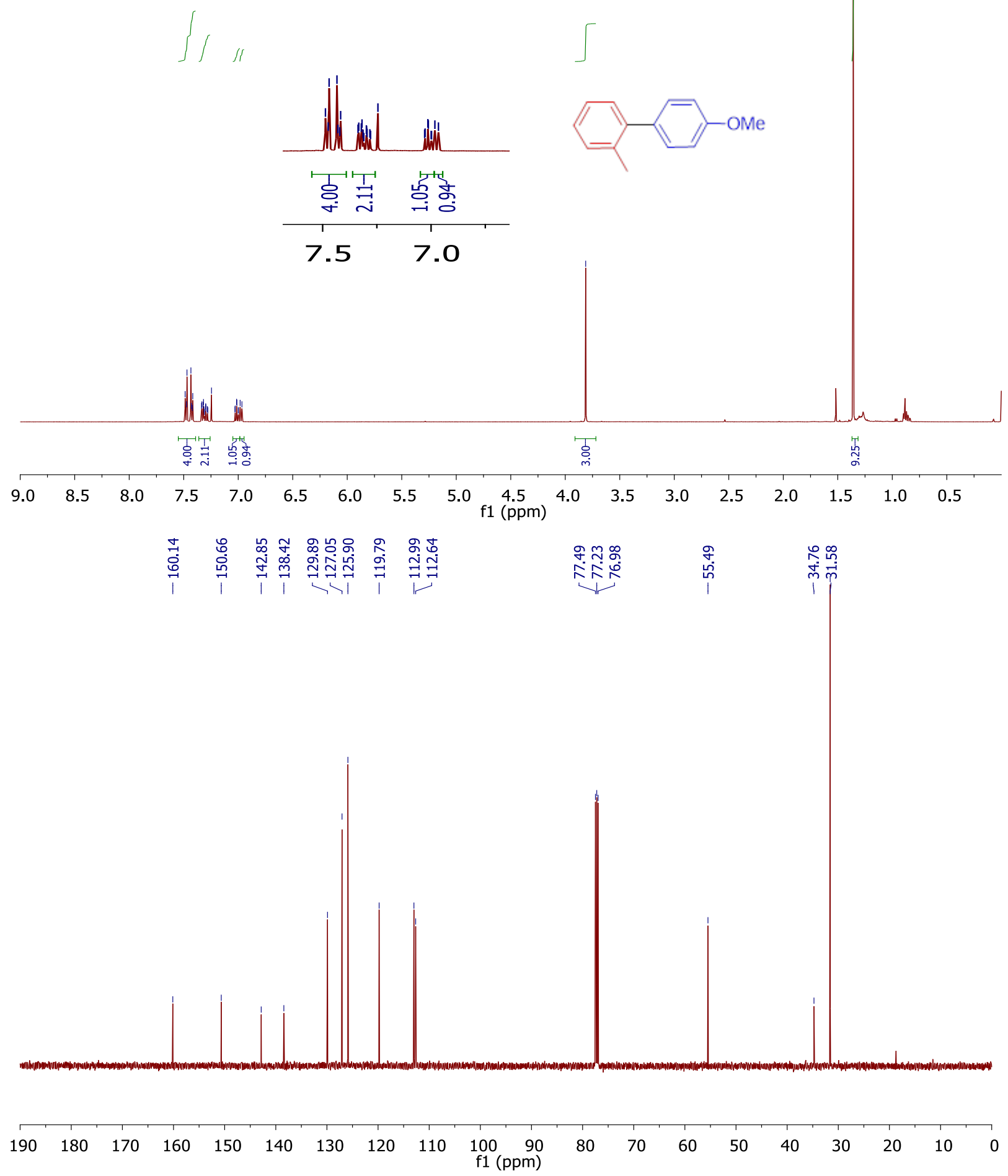




\section{${ }^{1} \mathrm{H}$ and ${ }^{13} \mathrm{C}\left\{{ }^{1} \mathrm{H}\right\}$ NMR Spectra of compound $3 \mathrm{~h}$}

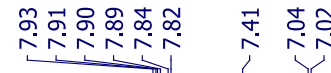
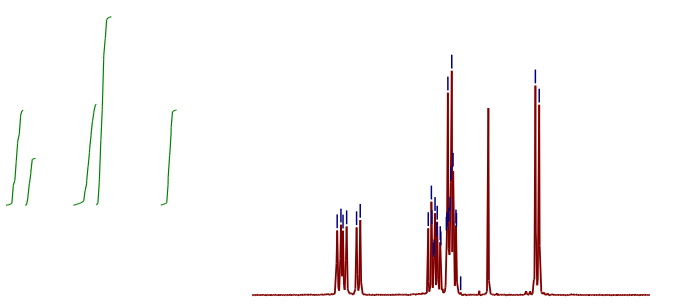

๓̊
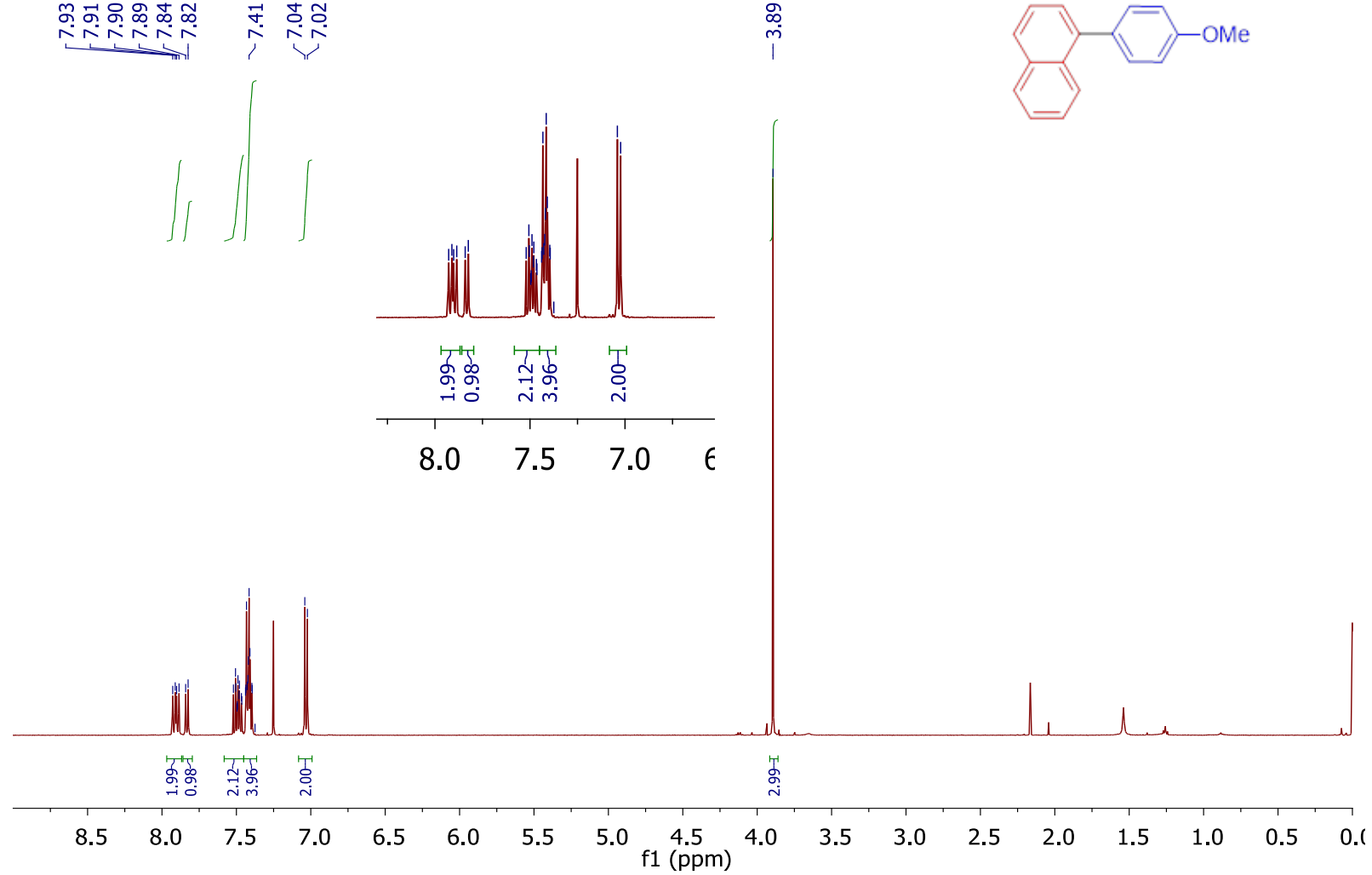

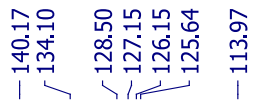

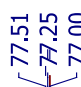

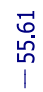

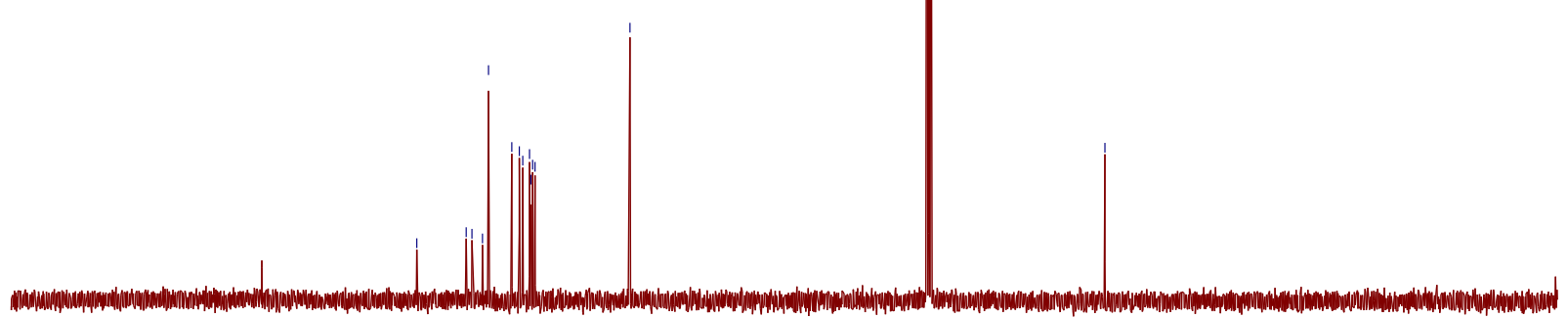

190

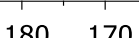

160

150

140

130120

110

f1 (ppm)

$\begin{array}{lllllllll}80 & 70 & 60 & 50 & 40 & 30 & 20 & 10 & 0\end{array}$ 


\section{${ }^{1} \mathrm{H}$ and ${ }^{13} \mathrm{C}\left\{{ }^{1} \mathrm{H}\right\}$ NMR Spectra of compound $3 \mathbf{i}$}

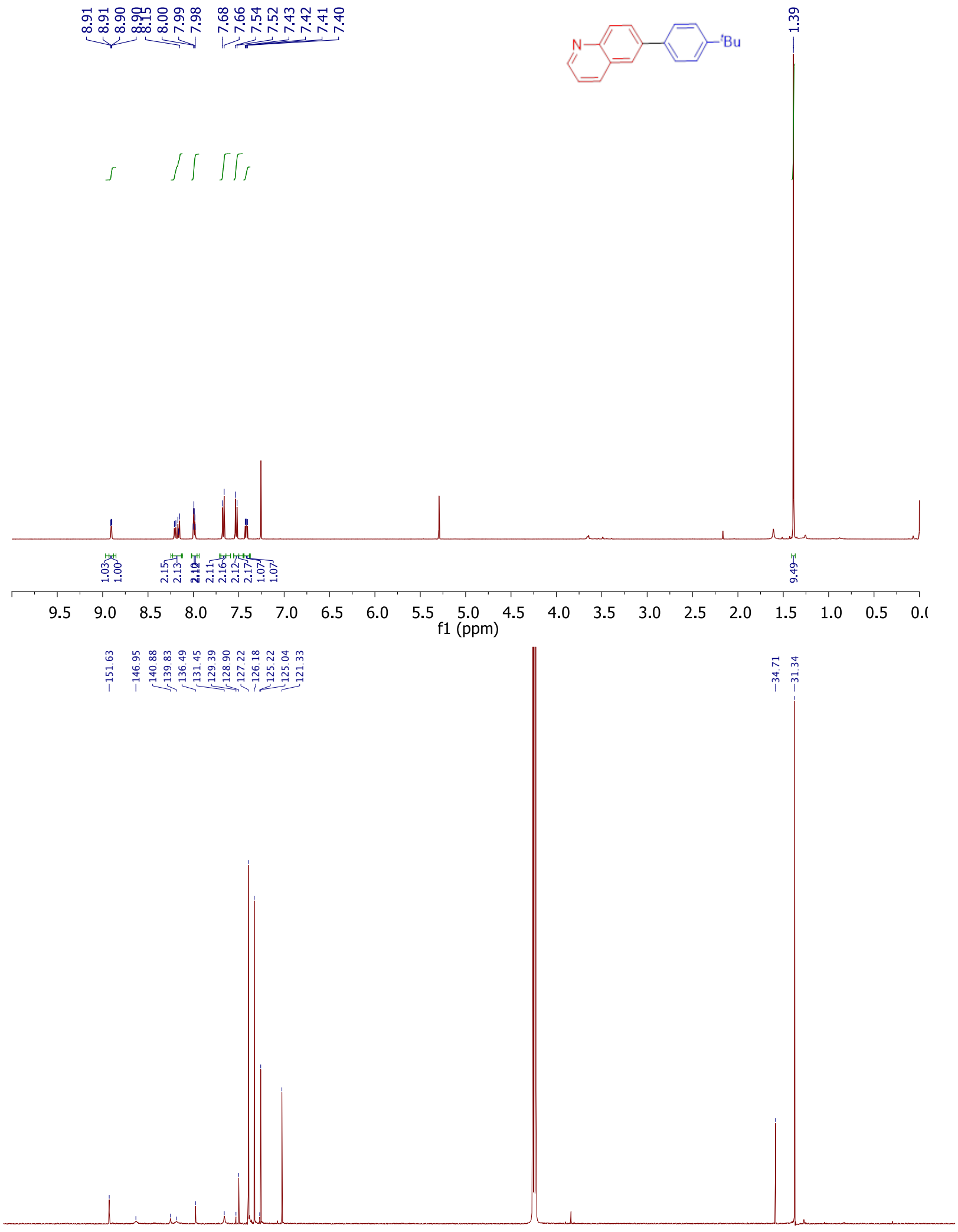




\section{$1 \mathrm{H}$ and ${ }^{13} \mathrm{C}\left\{{ }^{1} \mathrm{H}\right\}$ NMR Spectra of compound $3 \mathrm{j}$}
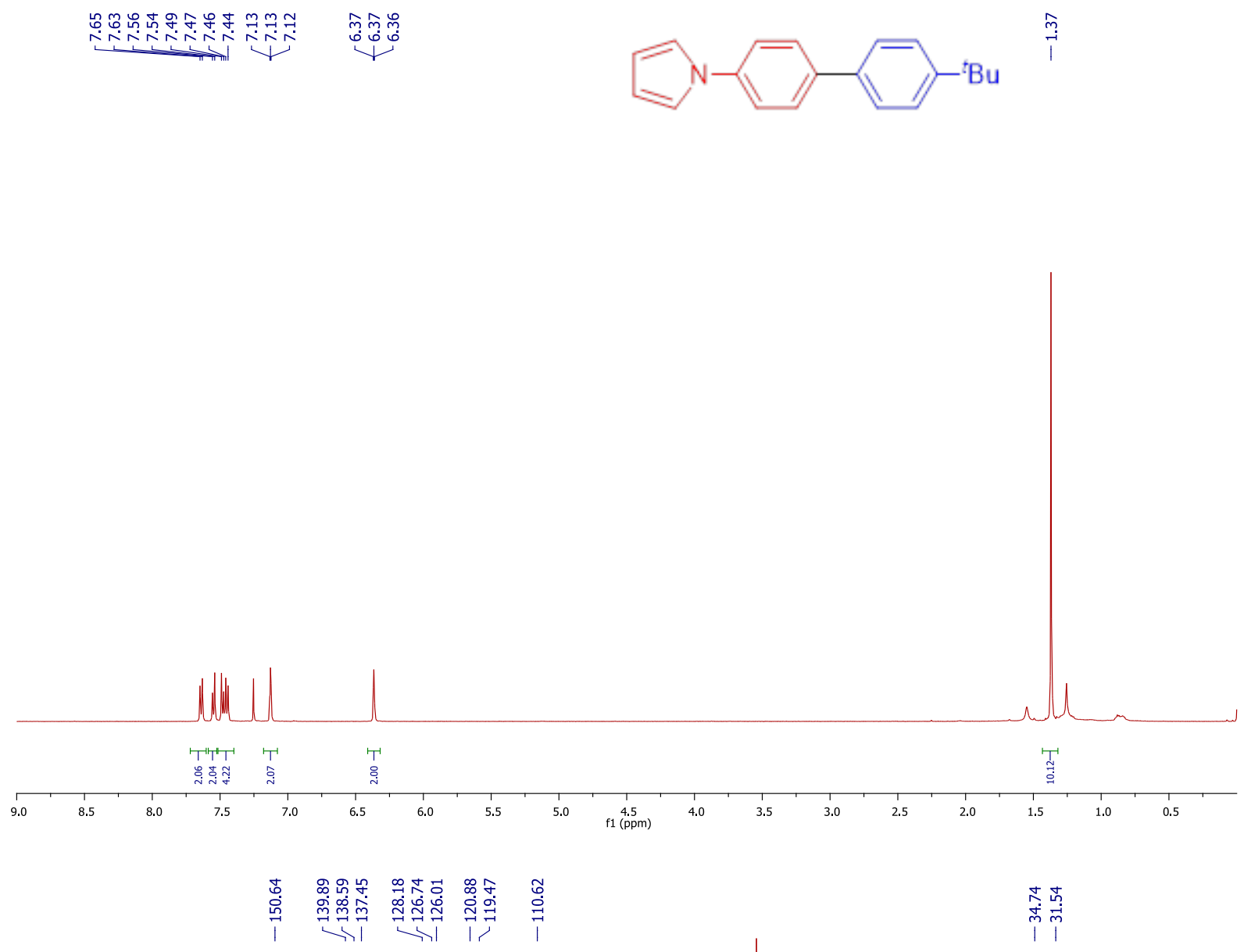

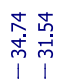
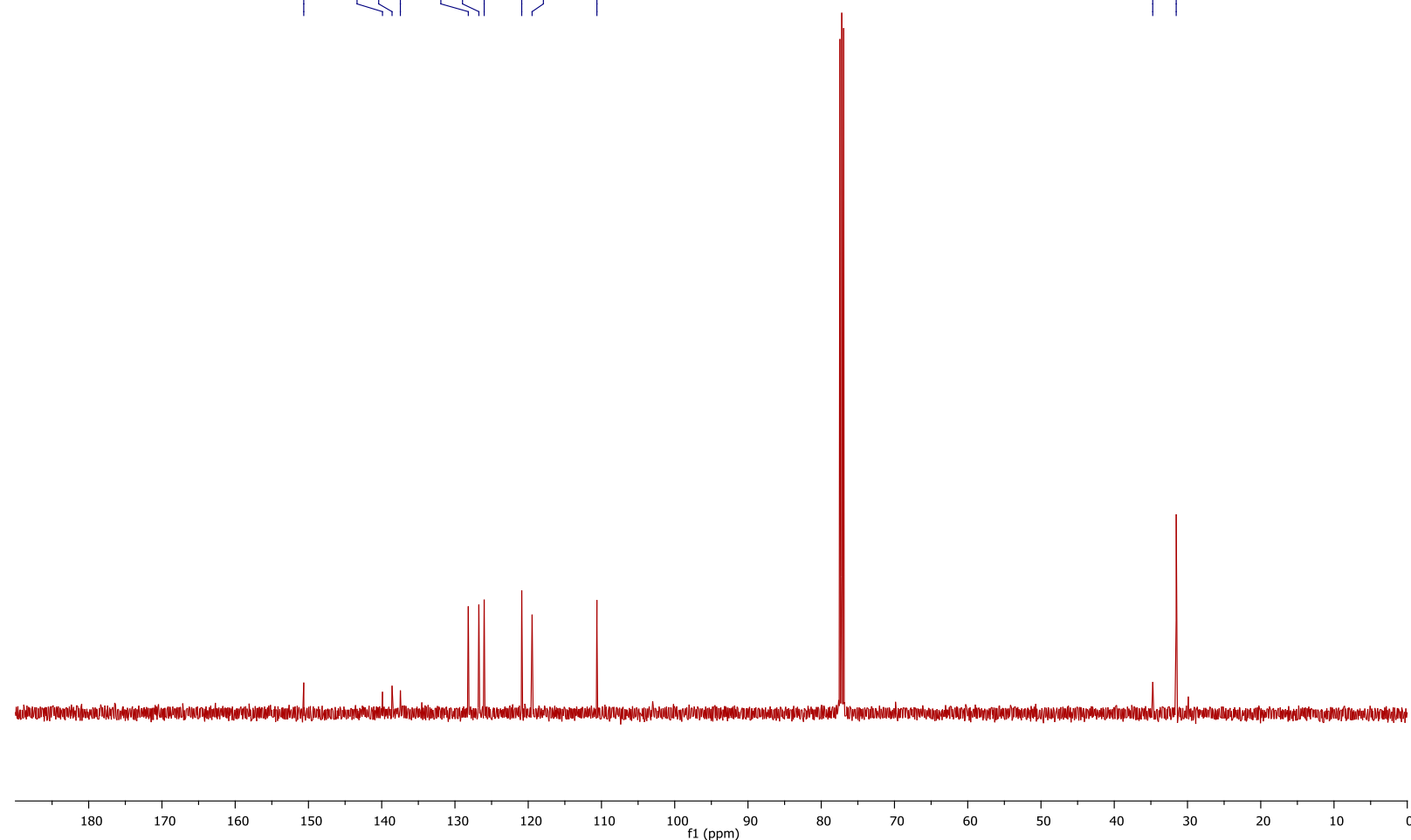

${ }_{\mathrm{f} 1(\mathrm{ppm})} 90$ 


\section{${ }^{1} \mathrm{H}$ and ${ }^{13} \mathrm{C}\left\{{ }^{1} \mathrm{H}\right\}$ NMR Spectra of compound $3 \mathrm{k}$}

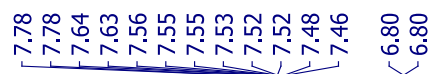

\section{ஸ}
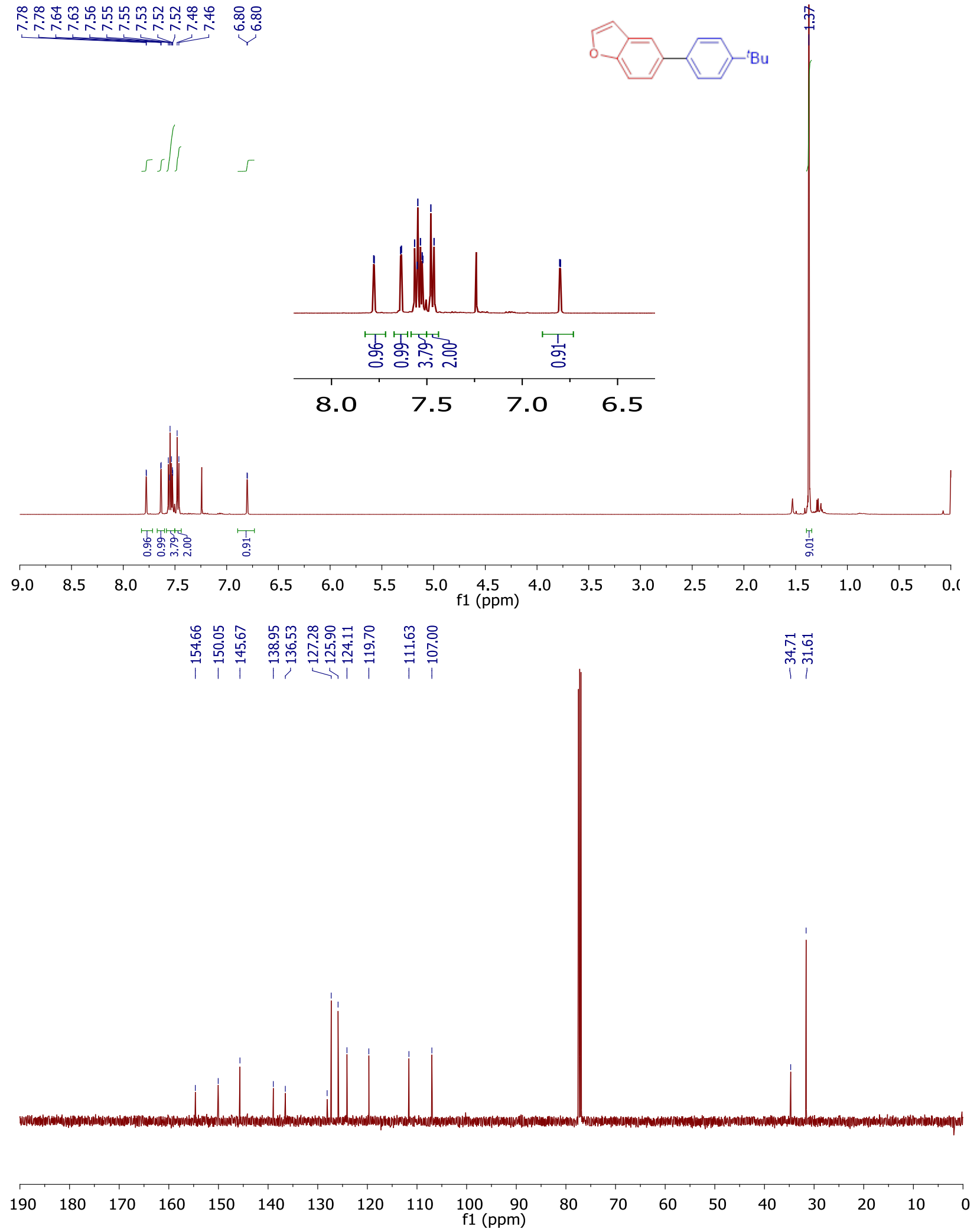


\section{${ }^{1} \mathrm{H}$ and ${ }^{13} \mathrm{C}\left\{{ }^{1} \mathrm{H}\right\}$ NMR Spectra of compound $3 \mathrm{I}$}

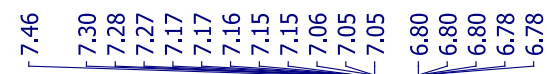

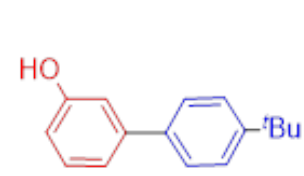

$\iint / 25 \tau$
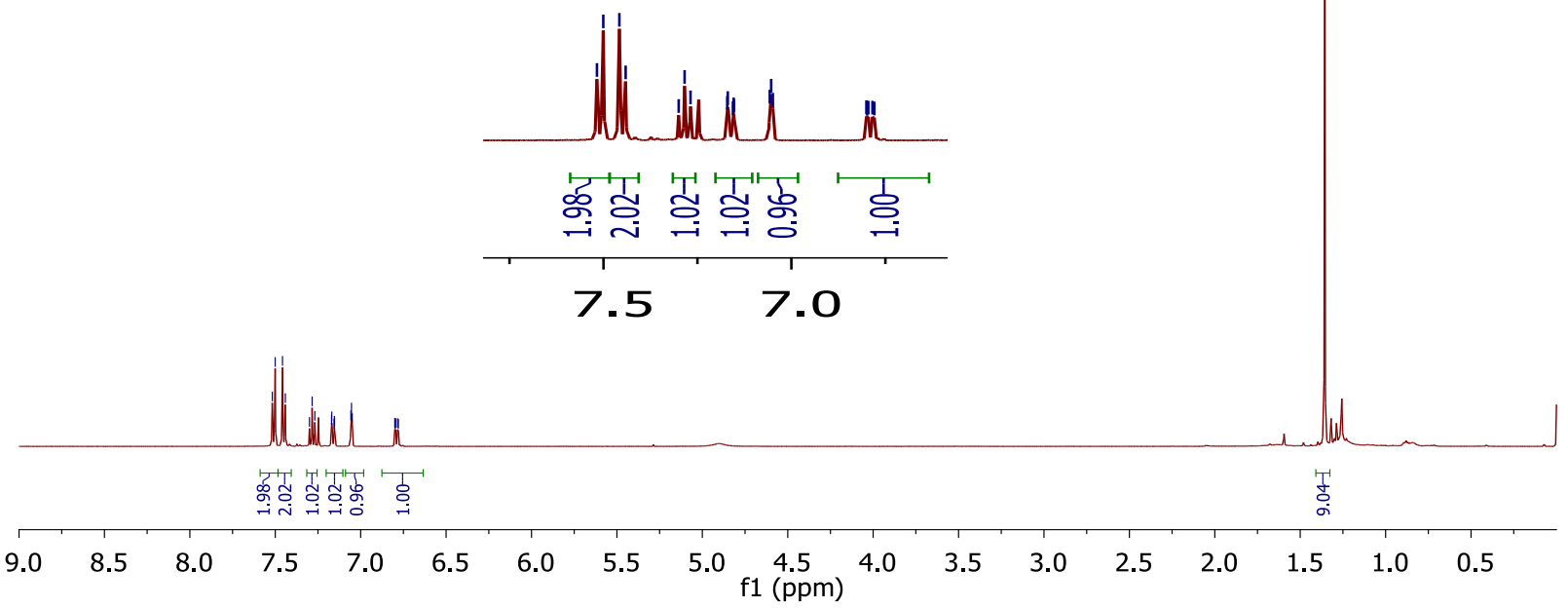

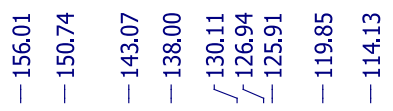

望

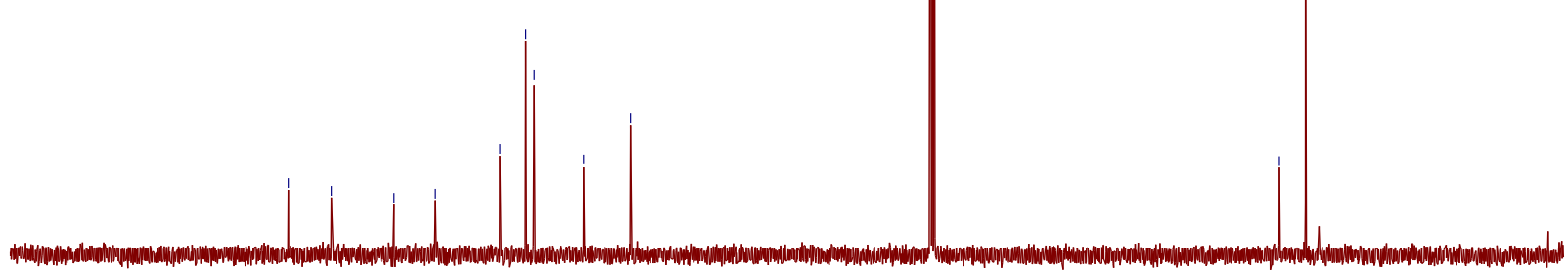

190

$150 \quad 140$

130

100
$\mathrm{f1}(\mathrm{ppm})$

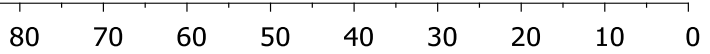




\section{${ }^{1} \mathrm{H}$ and ${ }^{13} \mathrm{C}\left\{{ }^{1} \mathrm{H}\right\}$ NMR Spectra of compound $3 \mathrm{~m}$}

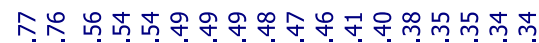

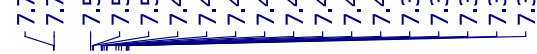
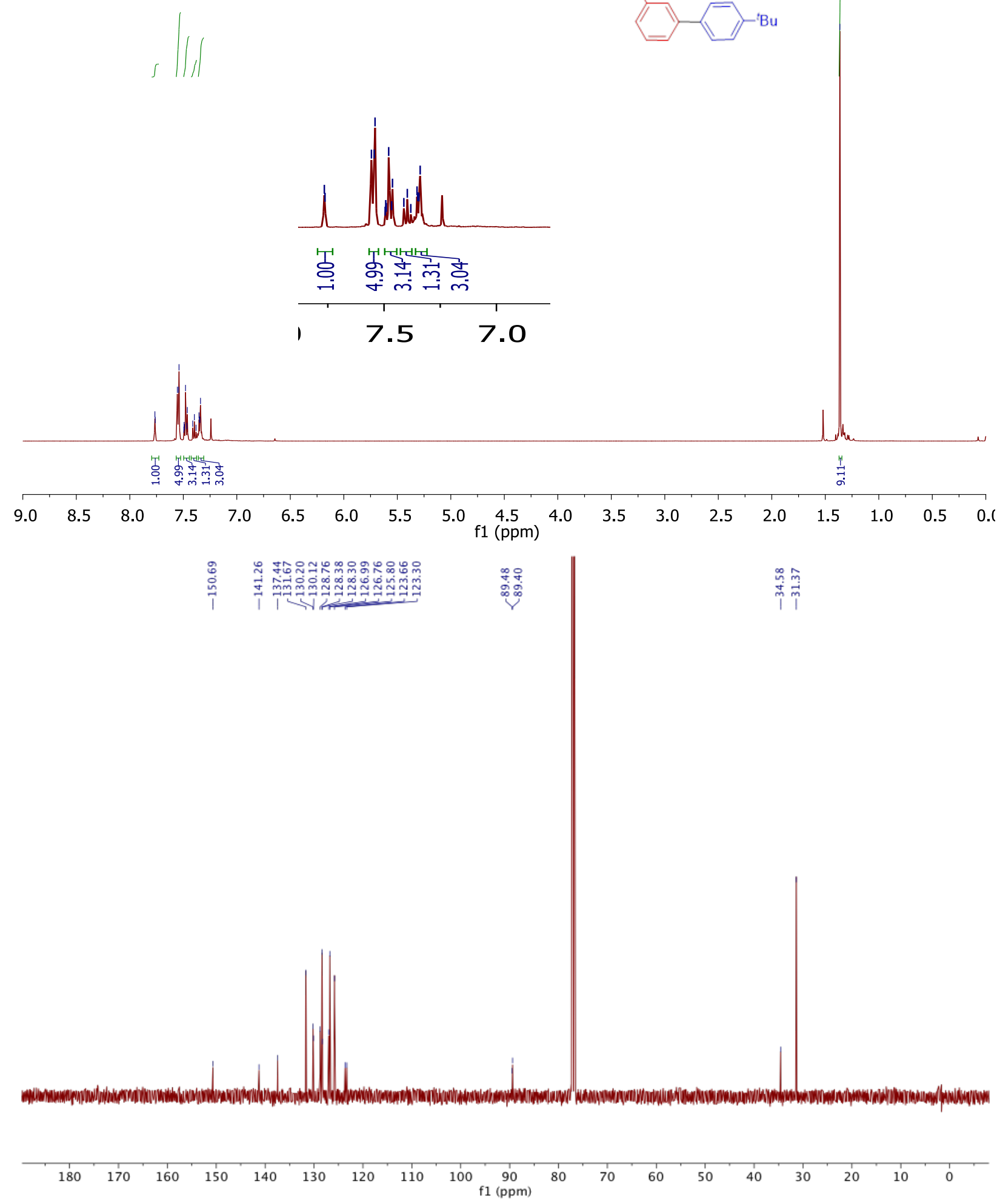
${ }^{1} \mathbf{H},{ }^{13} \mathrm{C}\left\{{ }^{1} \mathbf{H}\right\}$ and ${ }^{31} \mathbf{P}\left\{{ }^{1} \mathbf{H}\right\}$ NMR Spectra of (2-benzylphenyl)dicyclohexylphosphane
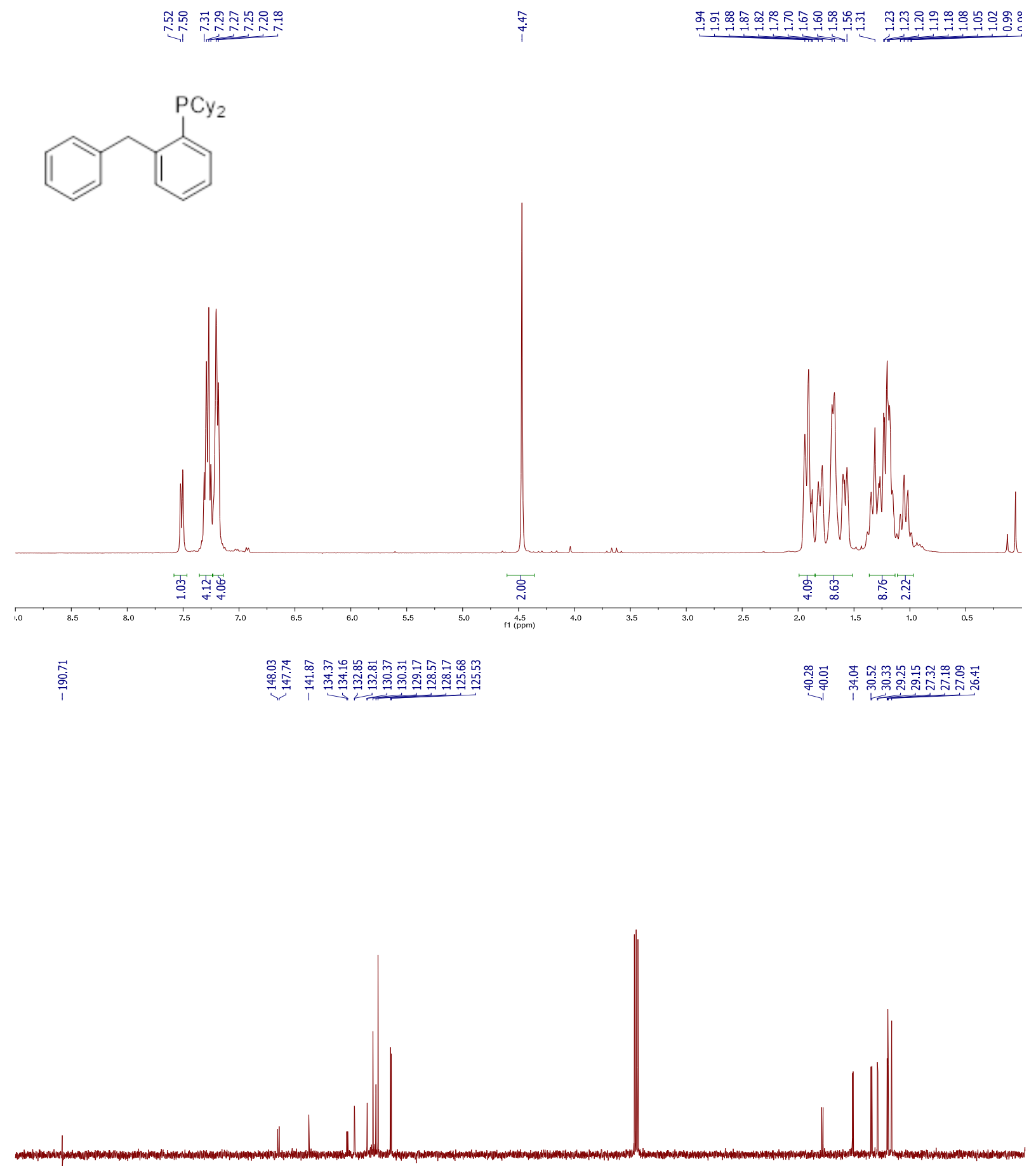


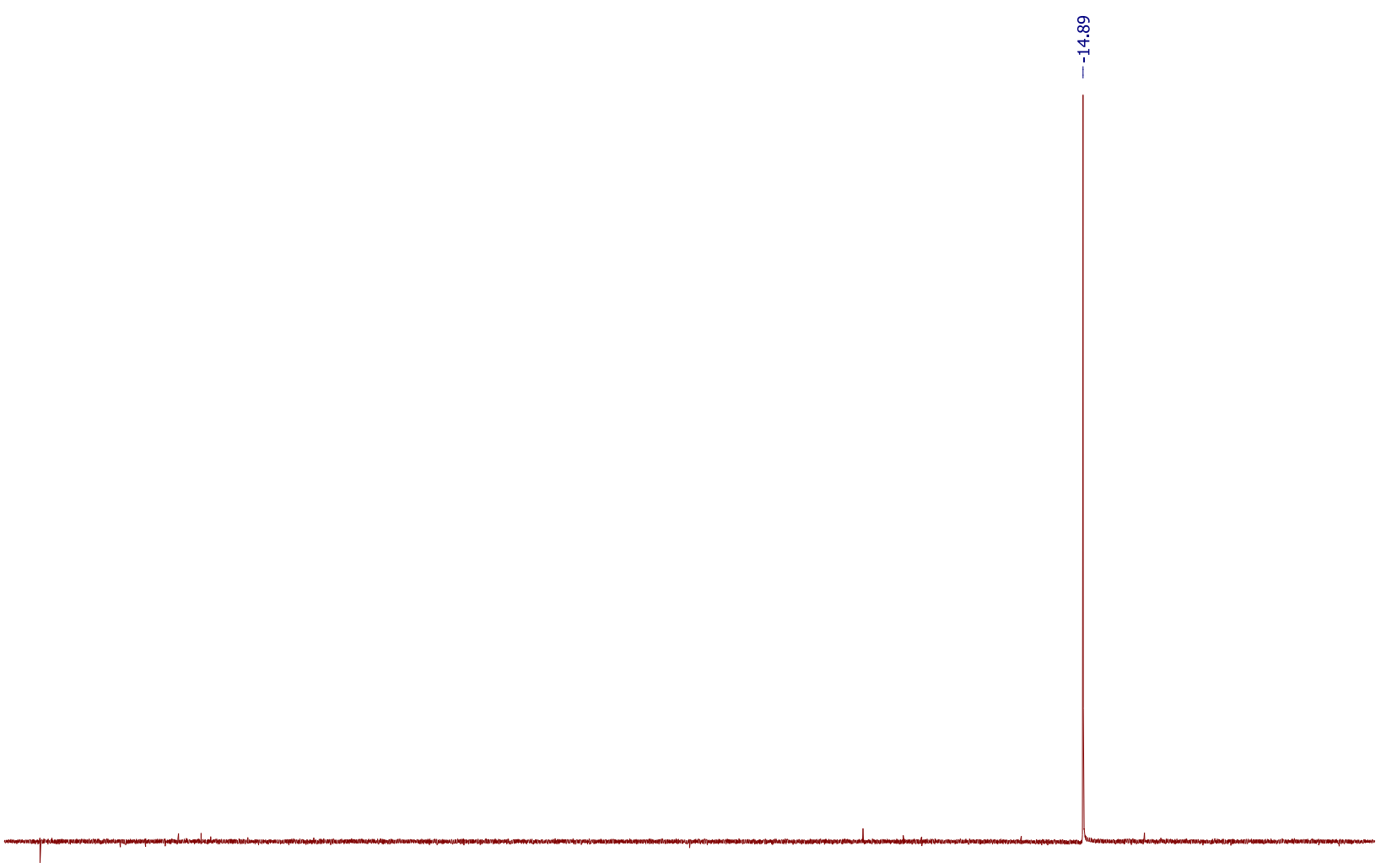

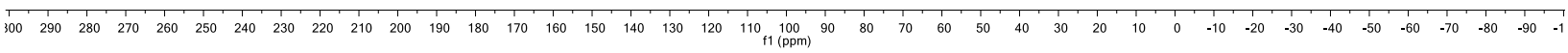




\section{${ }^{1} \mathrm{H},{ }^{13} \mathrm{C}\left\{{ }^{1} \mathrm{H}\right\}$ and ${ }^{31} \mathrm{P}\left\{{ }^{1} \mathrm{H}\right\}$ NMR Spectra of (2-benzylphenyl)diphenylphosphane}

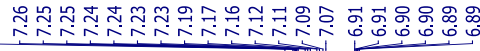

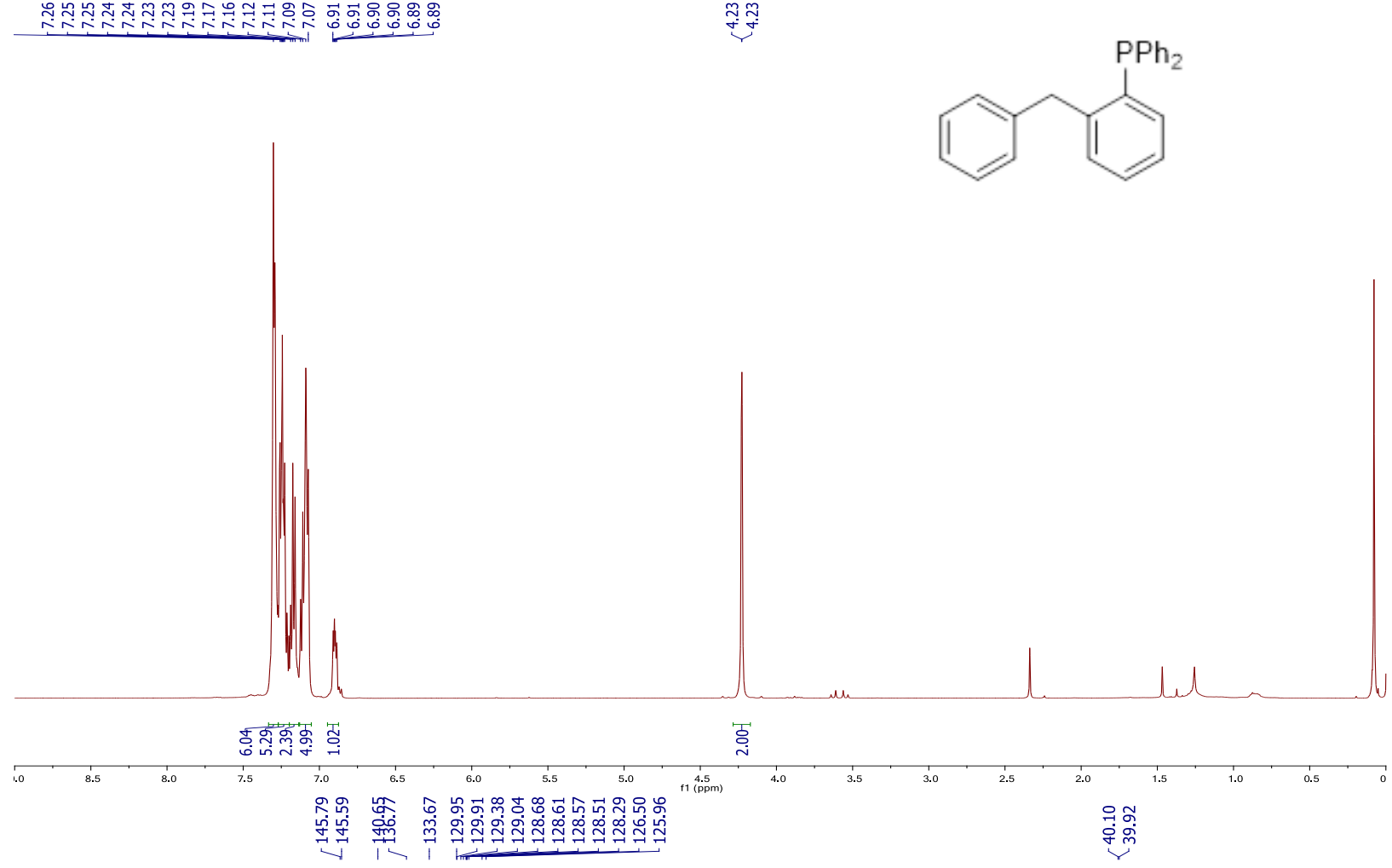

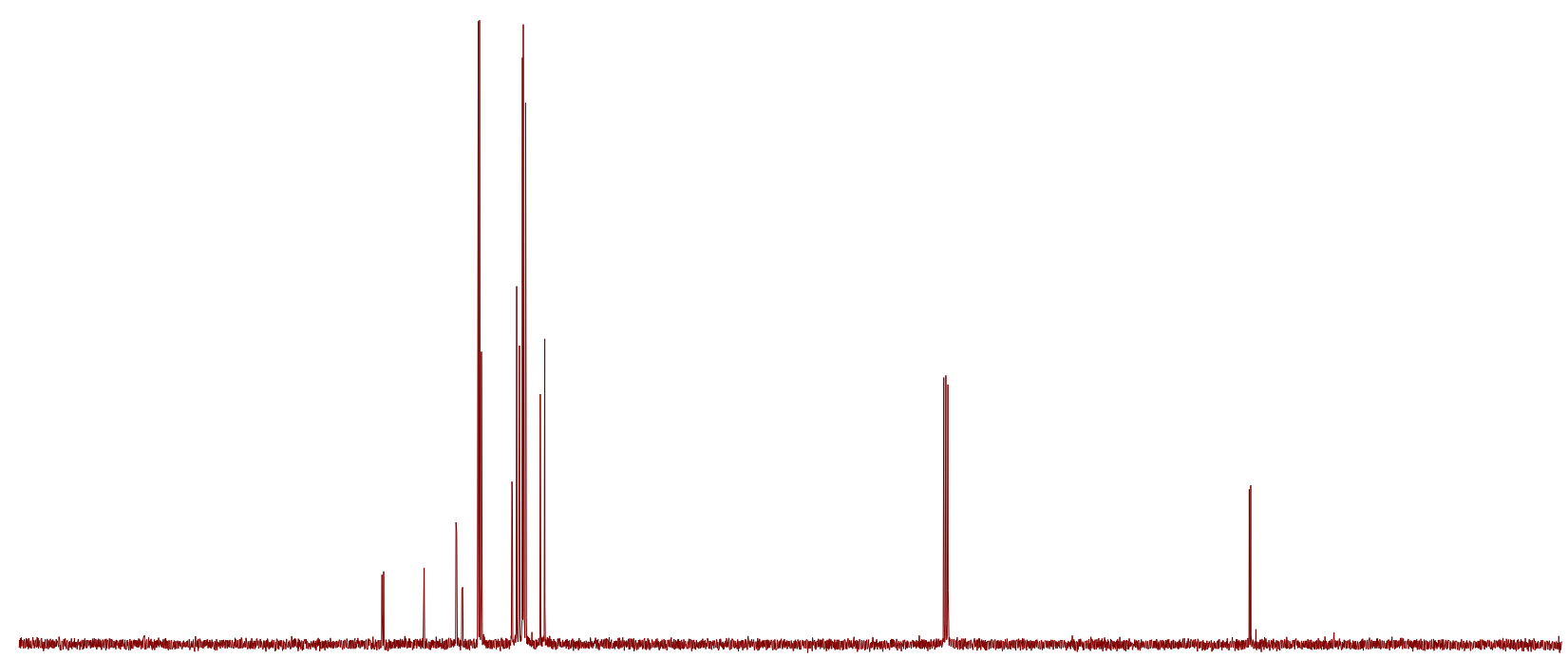




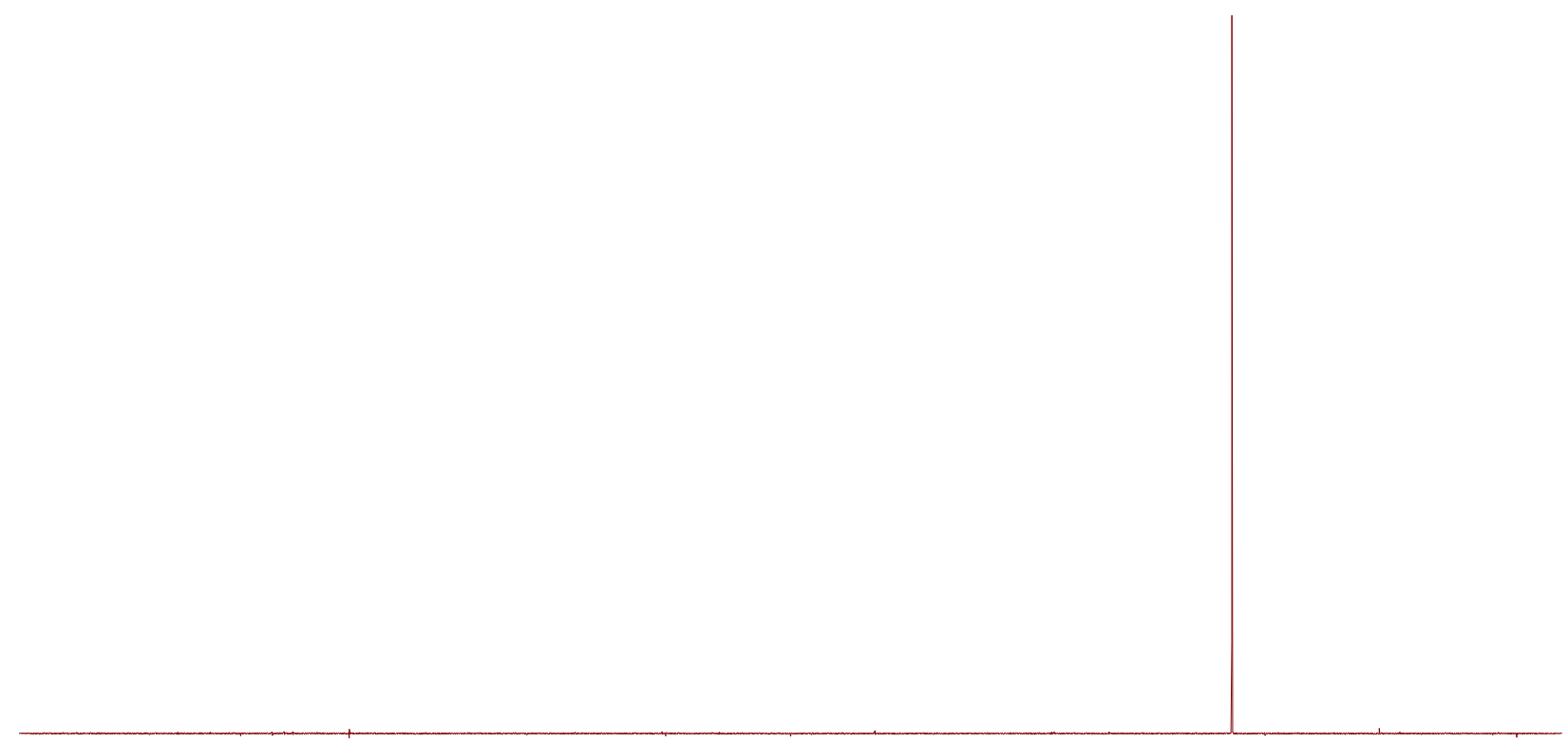

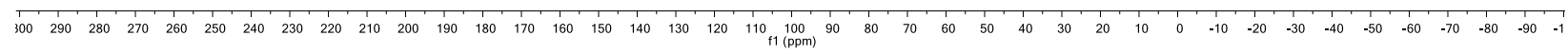




\section{${ }^{1} \mathrm{H}$ and ${ }^{13} \mathrm{C}\left\{{ }^{1} \mathrm{H}\right\}$ NMR Spectra of dicyclohexyl(o-tolyl)phosphane}
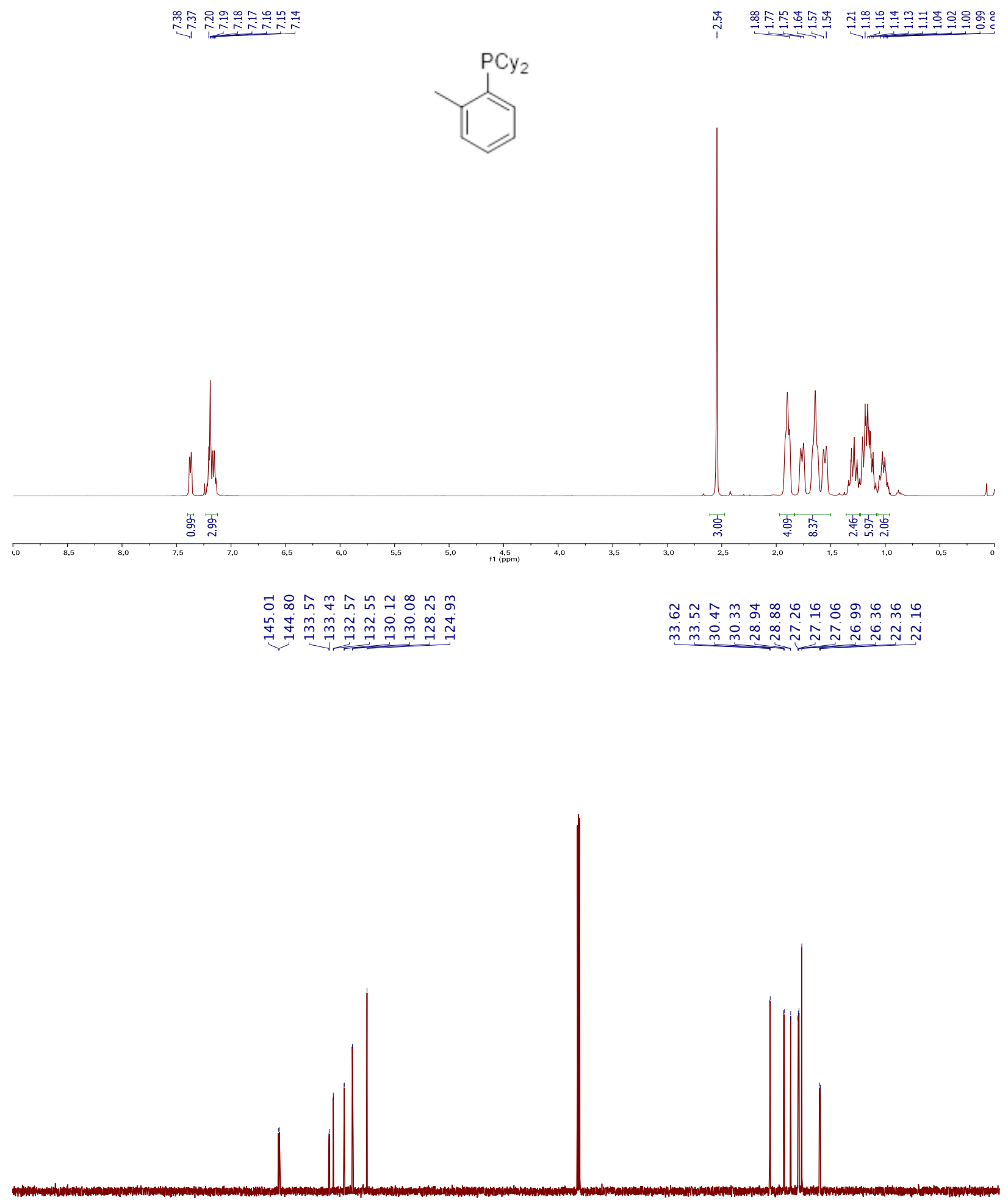


\section{${ }^{1} \mathrm{H},{ }^{13} \mathrm{C}\left\{{ }^{1} \mathrm{H}\right\}$ and ${ }^{31} \mathbf{P}\left\{{ }^{1} \mathrm{H}\right\}$ NMR Spectra of (3-benzylphenyl)dicyclohexylphosphane}

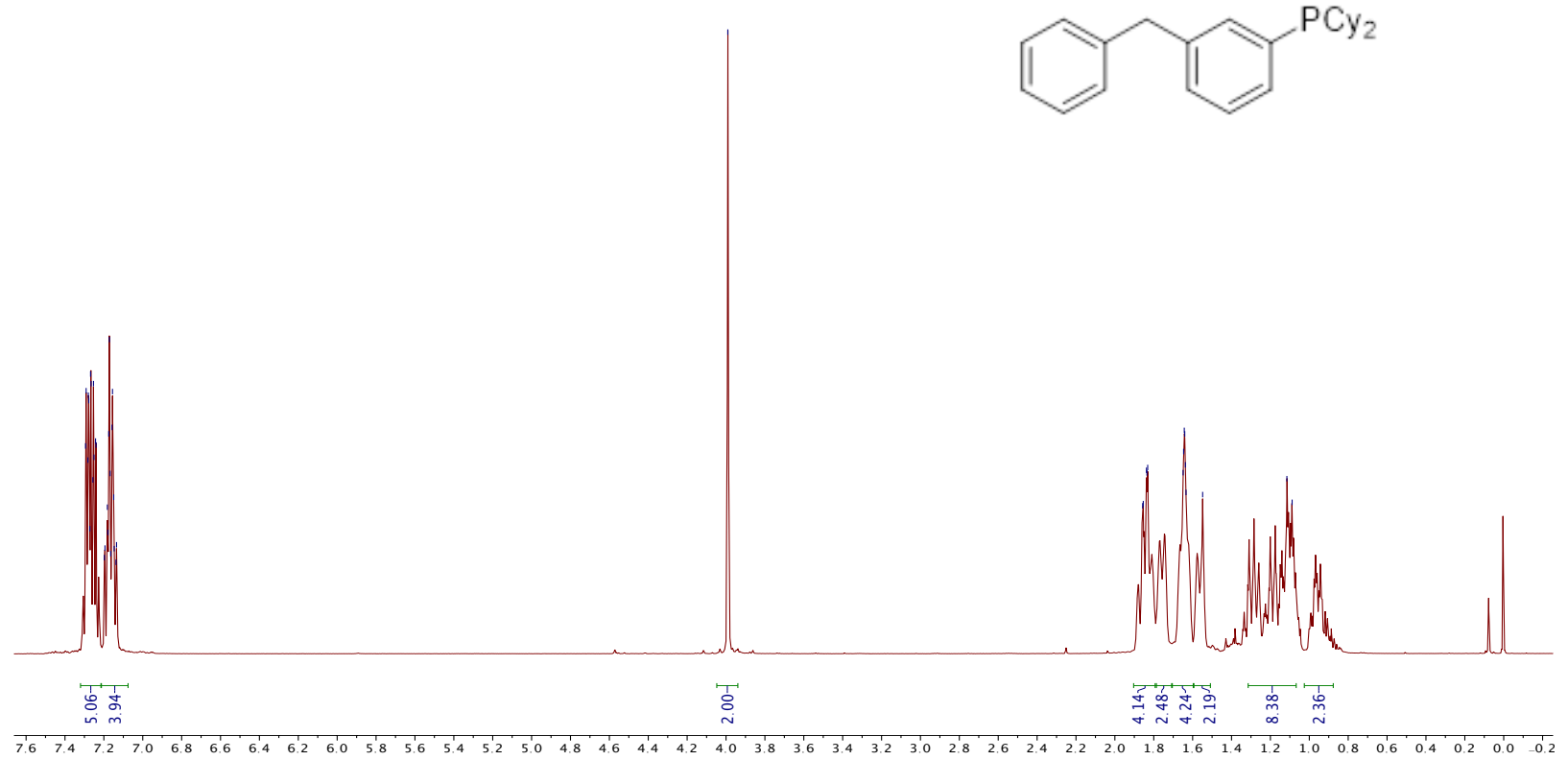

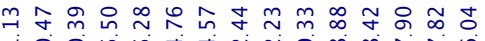

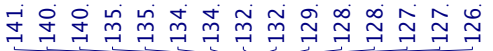

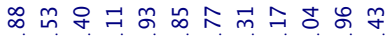

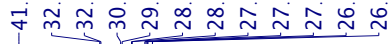

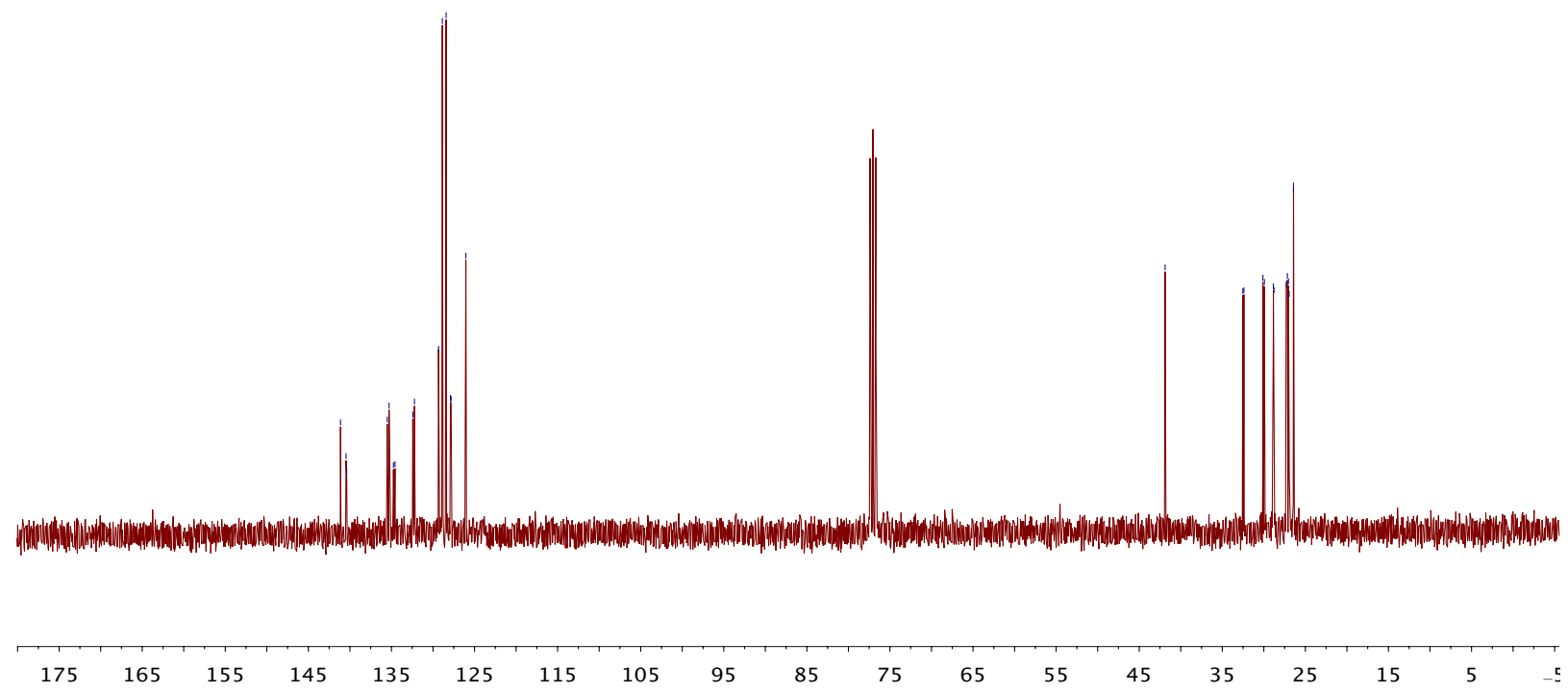




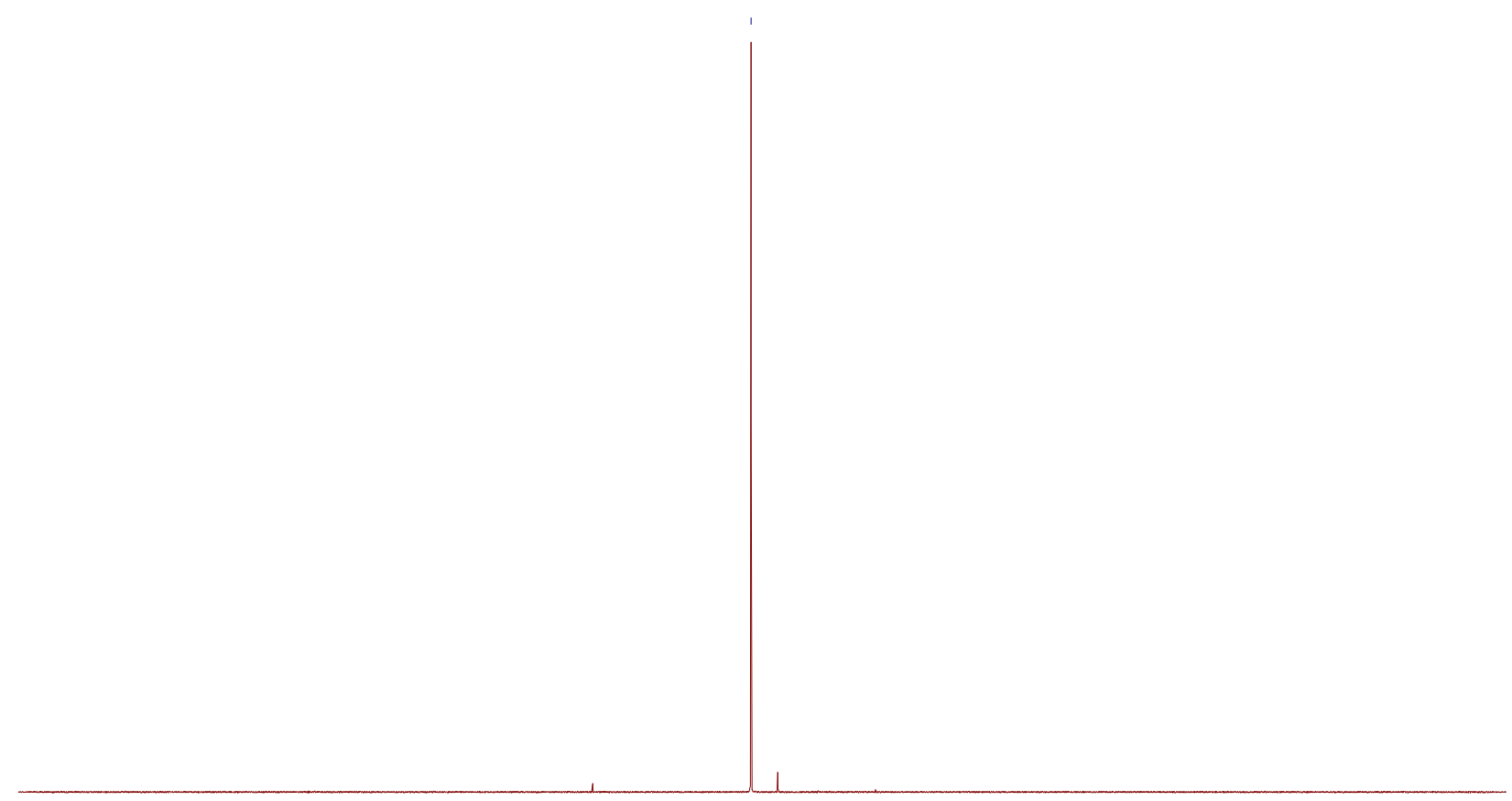

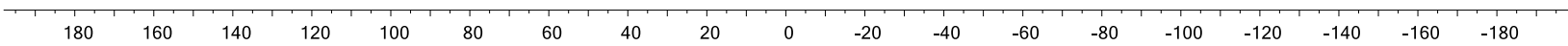




\section{Computational Methods}

\section{Experimental}

All density functional theory (DFT) calculations were performed with the ORCA program package, v3.0.3. ${ }^{12}$ Geometry optimizations were carried out at the unrestricted B97 level of DFT with dispersion correction (B97-D3). The def2-TZVP basis sets and the def2-TZVP/J auxiliary basis sets (used to expand the electron density in the resolution-of-identity (RI) approach) were used for all atoms except hydrogen. All hydrogens were described using the def2-SVP basis sets and def2-SV/J auxiliary basis sets. ${ }^{13}$ The def2-SD effective core potential and corresponding def2-TZVP/def2-TZVP/J basis sets (ibid.) were applied to $\mathrm{Pd}$, and the conductor-like screening model (COSMO; dipole moment corresponding to DME) was implemented as described in the results section. ${ }^{13}$

The SCF calculations were tightly converged $\left(1 \times 10^{-8} E_{\mathrm{h}}\right.$ in energy, $1 \times 10^{-7} E_{\mathrm{h}}$ in the density change, and $5 \times 10^{-7}$ in the maximum element of the DIIS error vector). In all cases the geometries were considered converged after the energy change was less than $1 \times 10^{-6} E_{\mathrm{h}}$, the gradient norm and maximum gradient element were smaller than $3 \times 10^{-4} E_{\mathrm{h}}-\mathrm{Bohr}^{-1}$ and $1 \times 10^{-4} E_{\mathrm{h}^{-}}$ $\mathrm{Bohr}^{-1}$, respectively, and the root-mean square and maximum displacements of all atoms were smaller than $6 \times 10^{-4}$ Bohr and $1 \times 10^{-3}$ Bohr, respectively. Numerical frequency calculations were used to verify that the calculated structures represented either local minima (ground states) or saddle points (transition states) on the potential energy surface. The reported energies are Gibbs free energies, calculated for $298.15 \mathrm{~K}$ and $1.00 \mathrm{~atm}$, as obtained from numerical frequency calculations on the optimized geometries.

Transition state searches were performed by educated and researched guesses of the transition state or by using relaxed coordinate/surface scans, involving successive geometry optimization calculations on species related by a regular change to fixed bond distances, bond angles, or torsion angles of interest, to provide suitable starting points for performing dedicated transition state optimization calculations. 


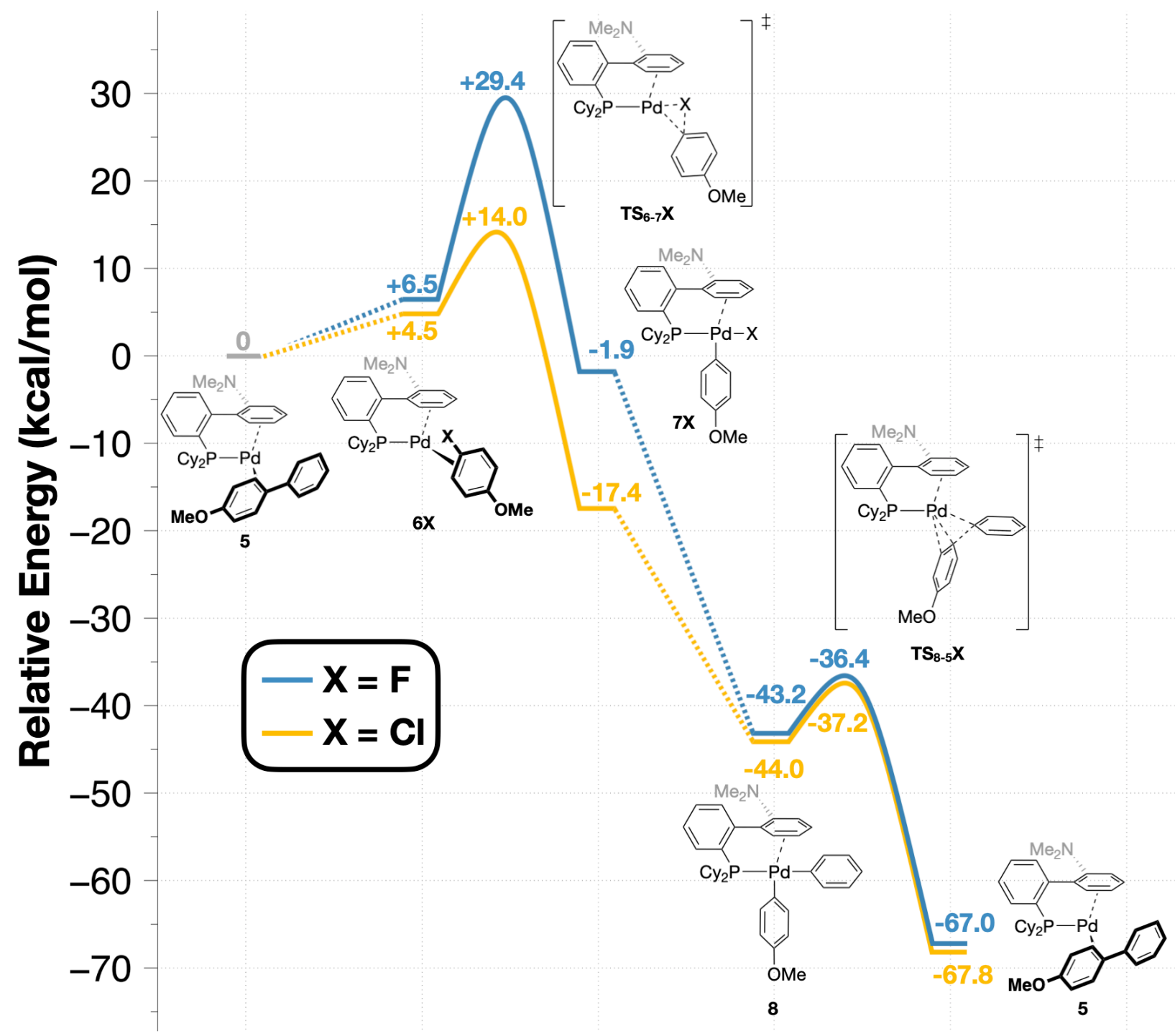

Figure S1. Potential energy diagram for the traditional KTC cross-coupling catalytic cycle. Dashed lines connect local minima; smooth lines connect local minima with transition states. 


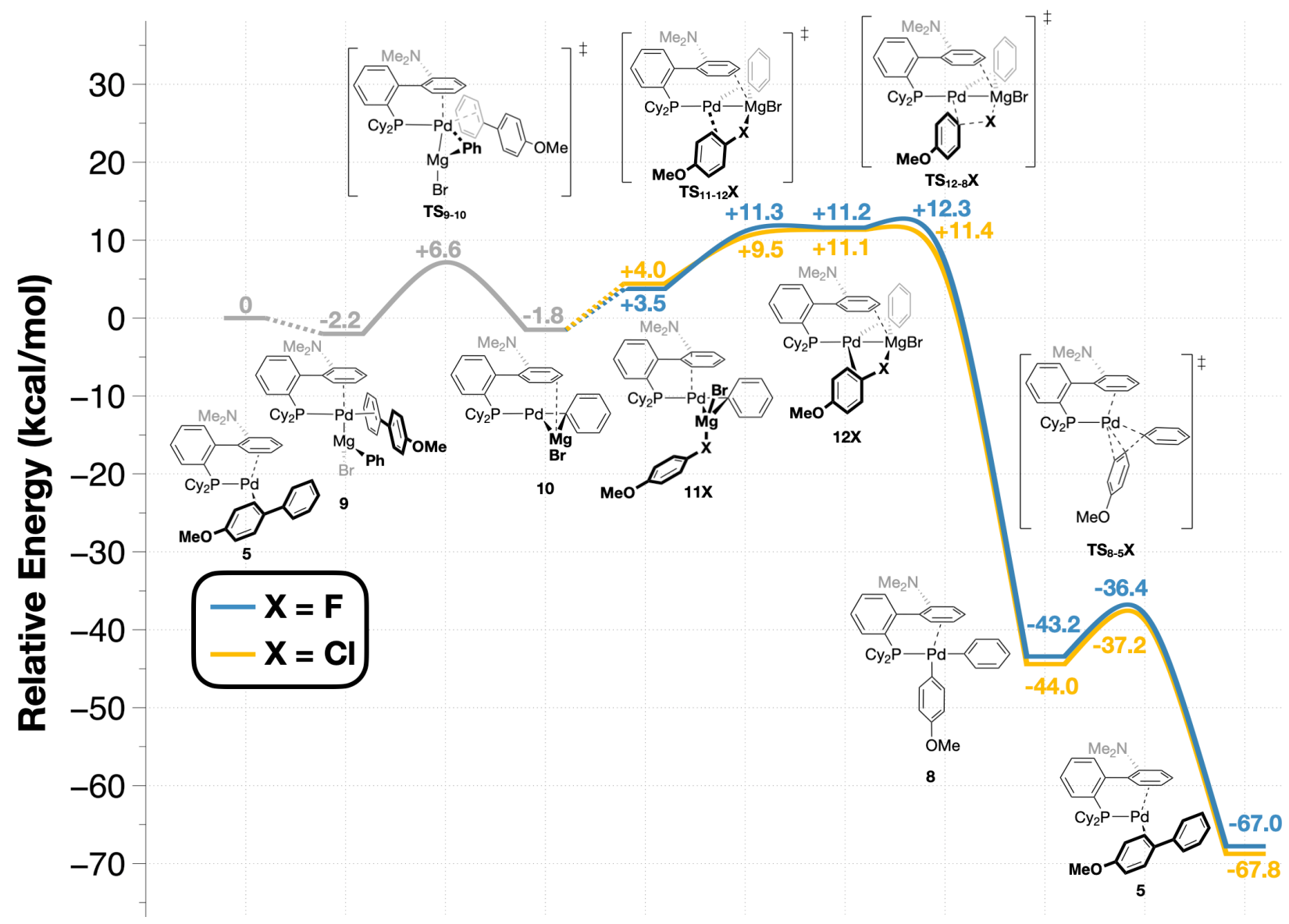

Figure S2. Potential energy diagram for the heterobimetallic KTC cross-coupling catalytic cycle. Dashed lines connect local minima; smooth lines connect local minima with transition states. 
Table S1. Relative energies for binding to Pd; all relative energies have included atoms in them and are similar comparisons, i.e. Pd-Davephos with aryl bound and DME coordinated will be compared to Pd-davephos with aryl bound and free DME

\begin{tabular}{|c|c|c|}
\hline Complex & Absolute Energy $\left(E_{\mathrm{h}}\right)$ & $\begin{array}{c}\text { Relative Energy } \\
\text { (kcal/mol) }\end{array}$ \\
\hline $\begin{array}{c}\text { Pd(0)-DavePhos complex } \\
\text { with Pd-aryl binding }\end{array}$ & -1534.49608039 & 0 \\
\hline $\begin{array}{c}\text { Pd(0)-DavePhos complex } \\
\text { without Pd-aryl binding }\end{array}$ & -1534.46861796 & -17.2 \\
\hline $\begin{array}{c}\text { Pd(0)-DavePhos complex } \\
\text { with Pd-aryl binding and } \\
\text { DME bound }\end{array}$ & -1843.18123072 & -19.6 \\
\hline $\begin{array}{c}\text { Pd(0)-bisDavePhos } \\
\text { complex without Pd-aryl } \\
\text { binding }\end{array}$ & -2942.27140296 & -40.1 \\
\hline $\begin{array}{c}\text { Pd(0)-bisDavePhos } \\
\text { complex with Pd-aryl } \\
\text { binding }\end{array}$ & -2942.30397556 & -10.0 \\
\hline $\begin{array}{c}\text { Pd(0)-DavePhos complex } \\
\text { with Pd-aryl binding with 4- } \\
\text { methoxybiphenyl bound }\end{array}$ & -2111.99777796 & -11.0 \\
\hline $\begin{array}{c}\text { Pd(0)-DavePhos complex } \\
\text { with Pd-aryl binding with 4- } \\
\text { methoxychlorobenzene } \\
\text { bound }\end{array}$ & -2340.74658017 & -9.0 \\
\hline $\begin{array}{c}\text { Pd(0)-DavePhos complex } \\
\text { with Pd-aryl binding with 4- } \\
\text { methoxyfluorobenzene } \\
\text { bound }\end{array}$ & -1980.33184320 & \\
\hline
\end{tabular}




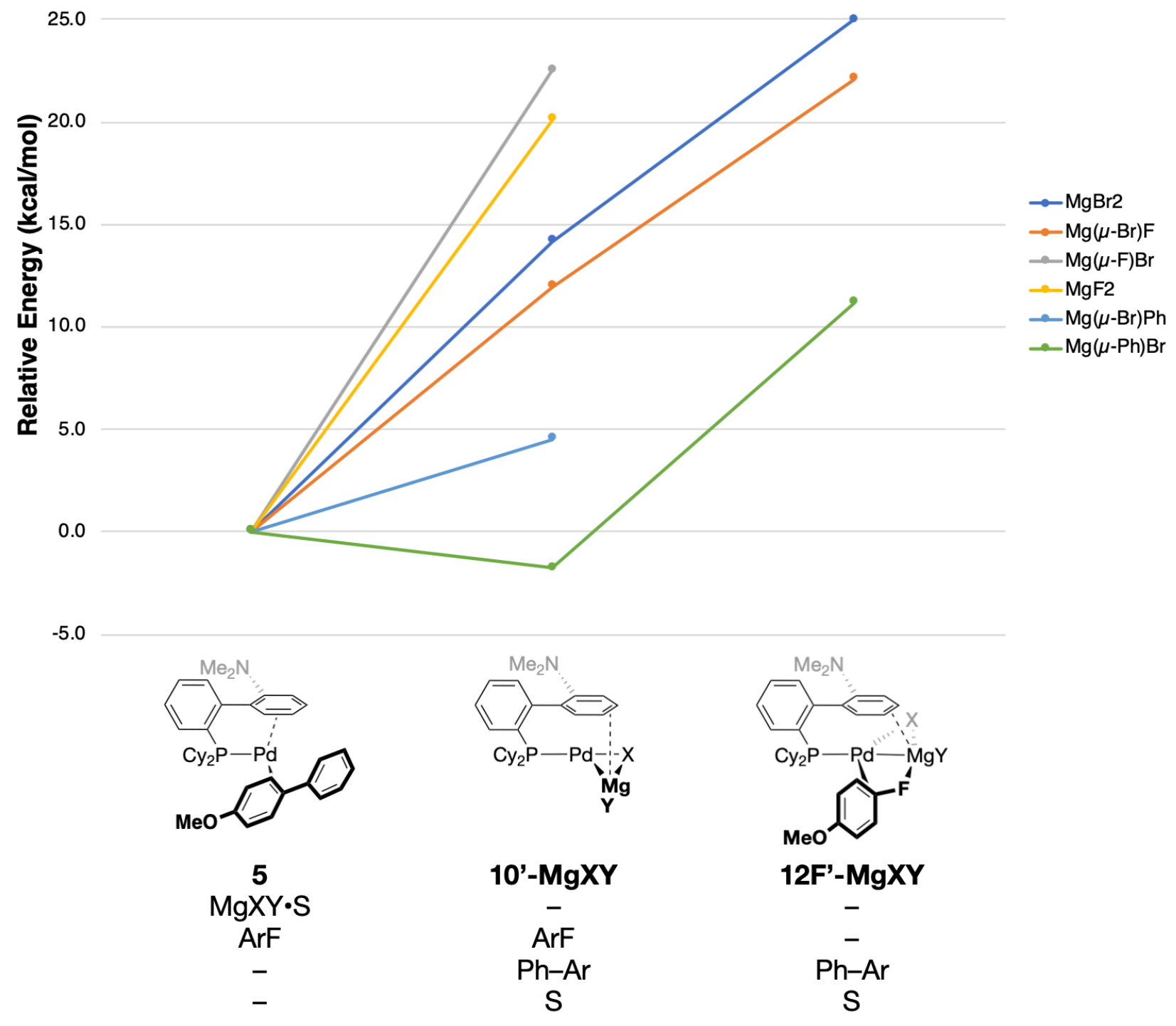

Figure S3. Comparison of the energy landscapes involving 5, 10'-MgXY, and 12F'-MgXY. S is the solvent (dme). All $\mathrm{Mg}$ salt were found to bind 2 equiv of dme, except $\mathrm{MgF}_{2}$, which bound 1 equiv. 


\section{Optimized Coordinates}

\section{DME}

Final Gibbs free enthalpy $\left(\Delta \mathrm{G}=\Delta \mathrm{H}-\mathrm{T}^{*} \Delta \mathrm{S}\right)=-308.68556105 E_{\mathrm{h}}$

$\begin{array}{llcc}\mathrm{O} & -0.61673357237257 & 0.97710425674099 & -0.00048536852404 \\ \mathrm{C} & 0.80519384896689 & 0.93433375549746 & 0.00013502537945 \\ \mathrm{H} & 1.09595712156975 & -0.12034753971188 & -0.00215347149471 \\ \mathrm{H} & 1.21922876646188 & 1.42876001949292 & -0.89375385966581 \\ \mathrm{H} & 1.21831290116084 & 1.42461014151089 & 0.89672666637206 \\ \mathrm{C} & -1.10945385600943 & 2.31144299042109 & 0.00232098988318 \\ \mathrm{H} & -0.75501521984746 & 2.85625886542313 & -0.88919132273899 \\ \mathrm{H} & -0.75523193945921 & 2.85239026947361 & 0.89625788253568 \\ \mathrm{C} & -2.62711507471204 & 2.24692776386809 & 0.00197262065552 \\ \mathrm{H} & -2.98153374857232 & 1.70268689974400 & 0.89384663181459 \\ \mathrm{H} & -2.98124089589178 & 1.70530118656862 & -0.89160713534424 \\ \mathrm{O} & -3.11998230236094 & 3.58117223750197 & 0.00376584082689 \\ \mathrm{C} & -4.54191863885106 & 3.62366346026798 & 0.00370453224692 \\ \mathrm{H} & -4.95548272615735 & 3.13027455339905 & 0.89838968149824 \\ \mathrm{H} & -4.83288799366927 & 4.67829211235218 & 0.00480931265579 \\ \mathrm{H} & -4.95535316025594 & 3.13219175744989 & -0.89209095610051\end{array}$

\section{DavePhos}

Final Gibbs free enthalpy $\left(\Delta \mathrm{G}=\Delta \mathrm{H}-\mathrm{T}^{*} \Delta \mathrm{S}\right)=-1407.74406041 E_{\mathrm{h}}$

$\begin{array}{cccc}\mathrm{P} & -0.96595110001088 & 6.58957357985308 & 13.55339244250487 \\ \mathrm{~N} & -3.87101536758996 & 9.50119248158413 & 14.95866224935145 \\ \mathrm{C} & 0.69484929263261 & 6.65898578958071 & 12.66828086935167 \\ \mathrm{H} & 0.52760658409337 & 5.99732078789541 & 11.80628908419680 \\ \mathrm{C} & 1.82362283710266 & 6.02696525057861 & 13.49325994537887 \\ \mathrm{H} & 2.01814061013153 & 6.64541841530276 & 14.37974382382806 \\ \mathrm{H} & 1.52088586055450 & 5.03635067111497 & 13.85369936744157 \\ \mathrm{C} & 3.11247328337922 & 5.92487825991522 & 12.66808307715855 \\ \mathrm{H} & 3.91358202664648 & 5.48634040936982 & 13.27779634879436 \\ \mathrm{H} & 2.94308583547084 & 5.24291836745782 & 11.82137542563583 \\ \mathrm{C} & 3.54100592885822 & 7.29413592329304 & 12.13115502603666 \\ \mathrm{H} & 4.44166431630005 & 7.19466822089832 & 11.51137010980451 \\ \mathrm{H} & 3.80826567518836 & 7.94381070994733 & 12.97853309755173 \\ \mathrm{C} & 2.41400550268301 & 7.95191746710210 & 11.33018423623709 \\ \mathrm{H} & 2.71345227709061 & 8.95610875954174 & 11.00346847652701 \\ \mathrm{H} & 2.22561821806579 & 7.36394059112826 & 10.41924783733910 \\ \mathrm{C} & 1.11851691864345 & 8.03369839869428 & 12.14642972218308 \\ \mathrm{H} & 0.32263294550791 & 8.47447467431257 & 11.53683855906960 \\ \mathrm{H} & 1.27170997343290 & 8.70030785111440 & 13.00554314126498 \\ \mathrm{C} & -2.15876238353201 & 7.21713324589681 & 12.25394302226260 \\ \mathrm{H} & -2.04623417477280 & 8.29682601551944 & 12.12914485923314\end{array}$




\begin{tabular}{|c|c|c|c|}
\hline & -1.91866373949694 & 6.55166215789429 & \\
\hline & -0.92660705371133 & 6.80287902490724 & \\
\hline & -1.95301007179483 & 5.45713416641894 & 11.00167957492207 \\
\hline & -2.97415059257651 & 6.99121920488600 & \\
\hline & -2.86225579497149 & 8.07093068311869 & \\
\hline & -2.79012376751297 & 6.48883219388462 & 8.90684423292312 \\
\hline & -4.39655712617762 & 6.71013834515017 & 10.35368019222097 \\
\hline & -4.53860332836672 & 5.62217458947355 & 10.4414492 \\
\hline & -5.13037493469309 & & \\
\hline & -4.63849835624316 & & 11.717 \\
\hline & -5.64 & & \\
\hline & -4.592227 & 8.4 & 11.60960 \\
\hline & -3.586550 & & \\
\hline & -3.67 & & 12.8 \\
\hline & -3.7 & & \\
\hline C & -0.681836 & 7.906 & 5336 \\
\hline & 0.02169741314907 & 7.3721 & 15.941 \\
\hline H & 0.13 & & \\
\hline & 0.60 & & 16.9 \\
\hline H & 1.14 & & \\
\hline & 0.49 & 9.55 & 16.8 \\
\hline $\mathrm{H}$ & 0.96 & & \\
\hline & -0.26 & & \\
\hline $\mathrm{H}$ & -0.40 & & \\
\hline & -0.88 & & \\
\hline $\mathrm{C}$ & -1.73 & 10.0 & \\
\hline C & -1.08 & 84 & \\
\hline & 0.00 & 10.6 & 12.728 \\
\hline $\mathrm{C}$ & -1.77 & 405 & \\
\hline $\mathrm{H}$ & -1.2352 & 9620 & \\
\hline $\mathrm{C}$ & -3.16 & 9971 & \\
\hline & -3.73 & & \\
\hline $\mathrm{C}$ & -3.83 & 10.8 & \\
\hline & -4.91 & 4932 & 21896 \\
\hline $\mathrm{C}$ & -3.15 & 5562 & 05635 \\
\hline $\mathrm{C}$ & -3.55 & & 3871 \\
\hline $\mathrm{H}$ & -2.48 & & \\
\hline $\mathrm{H}$ & -4.03994507596745 & 9.18375946679761 & 17.01771817618276 \\
\hline $\mathrm{H}$ & -3.914696 & 7089 & \\
\hline $\mathrm{C}$ & -5.31453412272702 & 9.38138125636893 & 14.79254737885676 \\
\hline & -5.55954783801441 & 8.96891577835017 & 13.81217555948833 \\
\hline $\mathrm{H}$ & -5.84593021557073 & 10.34337779841112 & 14.91159855399863 \\
\hline $\mathrm{H}$ & -5.68789570859976 & 8.69486117285284 & 15.55930722772066 \\
\hline
\end{tabular}

cis-PhMgBr(dme)2

Final Gibbs free enthalpy $\left(\Delta \mathrm{G}=\Delta \mathrm{H}-\mathrm{T}^{*} \Delta \mathrm{S}\right)=-3624.54077266 E_{\mathrm{h}}$ 
Mg -7.73232836150590

$\mathrm{Br}-8.24129101069317$

C -5.81590044089506

H -6.73786066626090

H $\quad-5.82143205559335$

H -4.94228140597880

C -4.65816793084664

H -4.29654084947068

H $\quad-3.86345404917682$

C -5.05563585578800

H -5.36411716583939

H $\quad-4.21397169822909$

C -6.54695169780341

H $\quad-7.40141173542796$

H $\quad-5.70906738184944$

H $\quad-6.83985969958205$

C -6.02187969338508

H $\quad-5.69956620150462$

H $\quad-5.22300521865745$

H $\quad-6.26859222727312$

C -7.75422728386717

H $\quad-7.79734361317419$

H $\quad-7.13554019797829$

C -9.15730057025656

H -9.59964172555045

H $\quad-9.78215948524997$

C -10.39833077538645

H -10.24324320278434

H -11.01442501889783

H -10.87488958886408

C -8.96124636774477

C -9.57631828316588

H $\quad-9.43133977486622$

C -10.37219654207027

H -10.81739610073192

C -10.59716171332039

C -10.01763782726259

H -10.18370488499851

C -9.22086404314323

H -8.78355300840782

O $\quad-5.80418481760161$

O -6.16677305655033

O $\quad-7.18724443968992$

O $\quad-9.09247429432298$

H $\quad-11.21100803835327$
$-3.15199294541910$ $-2.42604973260827$ $-5.05911144712044$ $-5.63538230031617$ $-4.52644552292423$ $-5.71874535739222$ $-3.24963085670916$ $-3.22596169103721$ $-3.64276966906455$ $-1.86385569770617$ $-1.85674131755031$ $-1.17117169766520$ $-0.09612671189833$ 0.12567902011643 0.57346818215075 0.01597488407897 $-3.64066534026523$ $-4.49202342429020$ $-2.88703536344029$ $-3.97674128377644$ $-1.98682939553659$ $-2.19038321548370$ $-1.09418658885174$ $-1.79843527166547$ $-0.89388743426340$ $-2.66714123618049$ $-1.50222093185435$ $-1.39747010796034$ $-2.38741955417491$ $-0.60432183714650$ $-4.93714172026312$ $-5.57222222425927$ $-5.15162892047218$

$-6.71897271668926$

$-7.17068639199223$

$-7.28901913462947$ $-6.69520350949950$ $-7.12841563020350$ $-5.55166812841267$

$-5.12568532136283$ $-4.12640880559640$ $-1.45618491675626$ $-3.10662601186397$ $-1.66343016401783$ $-8.18280255802663$
2.04681847004802 4.60003401031295 3.60702746816349 3.50506475258961 4.56532447334873 3.53539812278316 2.48572690686093 1.45295361973619 3.12868557998919 2.93350087640253 3.98780558481226 2.78991259978100 2.38181486296312 1.74140363652603 2.14510261854644 3.43320582403583 $-0.76322154049410$ $-0.16073827223396$ $-0.81158038325190$ $-1.77826995624361$ $-0.82085280300679$ $-1.89934124132172$ $-0.64848788615713$ $-0.30062907969512$ $-0.73979058422619$ $-0.55337273385199$ 1.71433276278991 2.78974116862119 1.50972634463907 1.30092167611759 1.83887043374577 2.94220913676360 3.93979950173313 2.82629160883384 3.71324547313227 1.57018575949639 0.44623533624198 $-0.54093744263738$ 0.59238179513639 $-0.31323096378130$ 2.50806027324134 2.11650958419047 $-0.13073684908413$ 1.12806276760098 1.46943670680197 


\section{4-Methoxychlorobenzene}

Final Gibbs free enthalpy $\left(\Delta \mathrm{G}=\Delta \mathrm{H}-\mathrm{T}^{*} \Delta \mathrm{S}\right)=-806.23300365 E_{\mathrm{h}}$

$\begin{array}{cccc}\text { C } & 0.89554158133468 & -1.13281561443441 & 0.27736788076000 \\ \mathrm{C} & 1.59316941495707 & 0.06134127394818 & 0.14381124994203 \\ \mathrm{C} & 0.90530088927997 & 1.27052741892752 & -0.04825903613975 \\ \mathrm{C} & -0.49458077606186 & 1.26893393122452 & -0.10539596581131 \\ \mathrm{C} & -1.19537146479293 & 0.06722374001979 & 0.02867307377614 \\ \mathrm{H} & 1.43152542177591 & -2.06515748906739 & 0.42731704675768 \\ \mathrm{H} & 2.67923435486305 & 0.07097827594745 & 0.18773884877768 \\ \mathrm{H} & -1.05421438249816 & 2.18529317092698 & -0.25404661273579 \\ \mathrm{H} & -2.28074364633589 & 0.06567122361160 & -0.01667459760293 \\ \mathrm{Cl} & -1.38394236195925 & -2.62830442036302 & 0.38775719382133 \\ \mathrm{O} & 1.68311088446507 & 2.38567539149480 & -0.16706862389450 \\ \mathrm{C} & 1.02690725321254 & 3.64460815425688 & -0.36128842661211 \\ \mathrm{H} & 0.44740442808213 & 3.64720165454580 & -1.29376714891237 \\ \mathrm{H} & 0.36764208114728 & 3.88058503535763 & 0.48502080459287 \\ \mathrm{H} & 1.82584676094119 & 4.38603815127997 & -0.42336044146606\end{array}$

\section{4-Methoxyfluorobenzene}

Final Gibbs free enthalpy $\left(\Delta \mathrm{G}=\Delta \mathrm{H}-\mathrm{T}^{*} \Delta \mathrm{S}\right)=-445.82137145 E_{\mathrm{h}}$
C $\quad-0.47302071633001 \quad-1.55561536304631$
0.07397776706690
C 0.90567276692614
$-1.62467118885734$
$-0.03992763016654$
C 1.64090389695471
$-0.43958111664580$
$-0.15402473135766$
C 0.98002849535923
0.79554908217757
$-0.15203964819901$
C -0.41865431314490
0.83626403127809
$-0.03567081056587$
C -1.15135092609964
$-0.34073588734521$
0.07791868161987
H 1.40807112013383
$-2.58803862256200$
$-0.04056738250301$
H 2.71938267288995
$-0.50173786193958$
$-0.24577839537794$
H -0.92202540133031
1.79938718144064
$-0.03536723417235$
H -2.23358550211433
$-0.31859795813635$
0.17121663554965
F -1.19140674596980
$-2.71317198131884$
0.18473217825176
O 1.60501738384340
2.00768479977192
$-0.25815641564966$
C 3.03158202168654
2.01532198249672
$-0.37367362052000$
H 3.31664531697992
3.06694738364587
$-0.44350887005335$
H 3.50250588449682
1.56299721994737
0.50978690683652
H 3.35791081571843
1.48428422909325
$-1.27853914075931$

\section{4-Methoxybiphenyl}

Final Gibbs free enthalpy $\left(\Delta \mathrm{G}=\Delta \mathrm{H}-\mathrm{T}^{*} \Delta \mathrm{S}\right)=-577.48571634 E_{\mathrm{h}}$
C -2.65646478279829
$-0.89397079605161$
$-0.30337204279326$
C -1.44382250717694
$-0.78077235586325$
0.38046612533960
C -0.85291428420286
0.46854886275871
0.56434798089617
C -1.45645725647981
1.63606260783954
0.06343705503068 


$\begin{array}{lllc}\mathrm{C} & -2.67780075636314 & 1.50533429914465 & -0.62232813881193 \\ \mathrm{C} & -3.27111620249610 & 0.25676085315203 & -0.80181780569493 \\ \mathrm{H} & -3.11786556235725 & -1.86825793104010 & -0.44549685575879 \\ \mathrm{H} & -0.96007377420061 & -1.66858092308346 & 0.78134267410334 \\ \mathrm{H} & 0.07941005087054 & 0.54247435894326 & 1.11835399110814 \\ \mathrm{H} & -3.15570972792644 & 2.38859416844106 & -1.03802638902988 \\ \mathrm{H} & -4.21296077189653 & 0.18188012009056 & -1.34055923290474 \\ \mathrm{C} & -0.82470534285628 & 2.96124683218919 & 0.24817501246043 \\ \mathrm{C} & -1.60019445040543 & 4.11911937071673 & 0.45290966898950 \\ \mathrm{C} & 0.56874166241851 & 3.11453585324191 & 0.22176861620531 \\ \mathrm{C} & -1.01246094866665 & 5.36471250827901 & 0.62319068248113 \\ \mathrm{H} & -2.68316693512977 & 4.03793746090456 & 0.50401114091752 \\ \mathrm{C} & 1.17724021341431 & 4.35937957106459 & 0.38900877811901 \\ \mathrm{H} & 1.20108161829654 & 2.24899647946333 & 0.04094991657386 \\ \mathrm{C} & 0.38477086195908 & 5.49675499459265 & 0.59219358687137 \\ \mathrm{H} & -1.62222635881208 & 6.24843954883812 & 0.79403374772483 \\ \mathrm{H} & 2.25872094231845 & 4.42932278170421 & 0.34696904413642 \\ \mathrm{O} & 0.87184719992330 & 6.76050257180535 & 0.76810110932505 \\ \mathrm{C} & 2.29284869342228 & 6.93895805571428 & 0.75758427325884 \\ \mathrm{H} & 2.72097538972580 & 6.64537502733245 & -0.21032944835610 \\ \mathrm{H} & 2.45804503400854 & 8.00526497064100 & 0.92505600531060 \\ \mathrm{H} & 2.76903864541084 & 6.36119453918120 & 1.56101170449780\end{array}$

\section{4 (Pd(0)-DavePhos complex with Pd-aryl binding)}

Final Gibbs free enthalpy $\left(\Delta \mathrm{G}=\Delta \mathrm{H}-\mathrm{T}^{*} \Delta \mathrm{S}\right)=-1534.49608039 E_{\mathrm{h}}$

$\begin{array}{cccc}\mathrm{Pd} & -0.65361359589378 & 4.71261018028524 & 15.02950468406577 \\ \mathrm{P} & -0.69550236867325 & 6.52367329105808 & 13.72437342412404 \\ \mathrm{~N} & -2.20476287263250 & 5.79764366149722 & 17.36255455576585 \\ \mathrm{C} & 0.85029232641385 & 6.74253297202420 & 12.70496023597532 \\ \mathrm{H} & 0.76331954320982 & 5.93848933465560 & 11.95825323446472 \\ \mathrm{C} & 2.07676206018471 & 6.42864013423251 & 13.57350061039281 \\ \mathrm{H} & 2.15326702298376 & 7.18619816189701 & 14.36551602815958 \\ \mathrm{H} & 1.93038796870301 & 5.46481507408842 & 14.07826468145416 \\ \mathrm{C} & 3.36251573465695 & 6.43016802785884 & 12.74467690478450 \\ \mathrm{H} & 4.22585223068001 & 6.24328686271574 & 13.39661336836651 \\ \mathrm{H} & 3.32444000504768 & 5.60237641941214 & 12.02004578485649 \\ \mathrm{C} & 3.54109455237761 & 7.75066988814625 & 11.98979797515590 \\ \mathrm{H} & 4.44404277389235 & 7.71519855427487 & 11.36658329226623 \\ \mathrm{H} & 3.68728054558271 & 8.56190042287873 & 12.71870684113838 \\ \mathrm{C} & 2.31449422885228 & 8.06797168993609 & 11.12969367616491 \\ \mathrm{H} & 2.43710713372302 & 9.03962458498479 & 10.63339793047442 \\ \mathrm{H} & 2.22308799531786 & 7.31241178021833 & 10.33475950067982 \\ \mathrm{C} & 1.02917574673136 & 8.07194094780533 & 11.96697559775998 \\ \mathrm{H} & 0.16665800943891 & 8.27947363226195 & 11.32257663154636 \\ \mathrm{H} & 1.08414543161420 & 8.88529644516093 & 12.70198444972861\end{array}$




\begin{tabular}{|c|c|c|c|}
\hline & -2.09736904418756 & & \\
\hline & -1.94355595312437 & 89535228810975 & \\
\hline & -2.14760555544268 & & \\
\hline & & & \\
\hline & -2.20247828995287 & & \\
\hline & -3.35748325799264 & 6.06701477360661 & \\
\hline & 3.24516744746627 & & \\
\hline & 3.3811362558 & & \\
\hline & -4.66 & & \\
\hline & -4.82 & & \\
\hline & -5.51 & & \\
\hline & -4.62 & & \\
\hline & -5.5 & & \\
\hline & -4.5 & & \\
\hline & -3.415670 & & \\
\hline & -3.5 & & \\
\hline & -3.38 & & \\
\hline y & -0.6 & & \\
\hline & -0.9 & & \\
\hline & -1.3 & & \\
\hline & -0.7 & & \\
\hline & -0.9 & & \\
\hline & -0.21 & & \\
\hline $\mathrm{H}$ & -0.0 & & \\
\hline & 0.05 & & \\
\hline & 0.43 & & \\
\hline & -0.1 & & \\
\hline & 0.13 & & \\
\hline & 1.47 & & \\
\hline & 2.20 & & \\
\hline C & & & \\
\hline & 2.98 & & \\
\hline & 1.01 & & \\
\hline & 1.33 & & \\
\hline & -0.36 & & \\
\hline $\mathrm{H}$ & -1.0 & & 491 \\
\hline & -0.86 & & \\
\hline $\mathrm{C}$ & -2.74 & & \\
\hline & -2.06 & & \\
\hline & -3.69 & & 7593999797 \\
\hline & -2.92986497255070 & 376050592 & 17627805452 \\
\hline $\mathrm{C}$ & -3.1711439245 & & \\
\hline & -2.99866917833634 & 3.85639502353230 & 17.68164761490504 \\
\hline & & & \\
\hline & -4.17497810807129 & 5.11511254406032 & 17.28771326565361 \\
\hline
\end{tabular}


(Pd(0)-DavePhos complex without Pd-aryl binding)

Final Gibbs free enthalpy $\left(\Delta \mathrm{G}=\Delta \mathrm{H}-\mathrm{T}^{*} \Delta \mathrm{S}\right)=-1534.46861796 E_{\mathrm{h}}$

\begin{tabular}{|c|c|c|c|}
\hline $\mathrm{Pd}$ & -1.40545533163390 & 4.67426420154200 & 14.25238831104184 \\
\hline $\mathrm{P}$ & -0.95900706241729 & 6.64938414993415 & 13.51527365050159 \\
\hline $\mathrm{N}$ & -3.86640175450471 & 9.50684077920226 & 14.92803284121242 \\
\hline $\mathrm{C}$ & 0.69256001059873 & 6.69311417700514 & 12.64694673680346 \\
\hline $\mathrm{H}$ & 0.51972840418795 & 6.04547494317504 & 11.77758064902225 \\
\hline $\mathrm{C}$ & 1.80083155268206 & 6.03546940663914 & 13.47598475391893 \\
\hline $\mathrm{H}$ & 1.97899741374564 & 6.62766898959287 & 14.38346725917512 \\
\hline $\mathrm{H}$ & 1.46600812056321 & 5.04226448585911 & 13.79894847127982 \\
\hline $\mathrm{C}$ & 3.10236995732395 & 5.94551488650213 & 12.67283779436467 \\
\hline $\mathrm{H}$ & 3.89099922026980 & 5.49407427696528 & 13.28914448916259 \\
\hline $\mathrm{H}$ & 2.94680912359223 & 5.27951210147821 & 11.81078365587935 \\
\hline $\mathrm{C}$ & 3.54446881126830 & 7.32278237415587 & 12.16839591083202 \\
\hline $\mathrm{H}$ & 4.45704350400404 & 7.23313049811948 & 11.56489223380803 \\
\hline $\mathrm{H}$ & 3.79449980372167 & 7.95831501723236 & 13.03154825448128 \\
\hline $\mathrm{C}$ & 2.43481458388327 & 7.99452548041025 & 11.35525367607609 \\
\hline $\mathrm{H}$ & 2.74220183626084 & 9.00196188695233 & 11.04706559291156 \\
\hline $\mathrm{H}$ & 2.26077851741617 & 7.41827120411145 & 10.43435053234857 \\
\hline $\mathrm{C}$ & 1.12543507701420 & 8.07344236318323 & 12.14936598051725 \\
\hline $\mathrm{H}$ & 0.34136633357225 & 8.52097068153042 & 11.52852832379797 \\
\hline $\mathrm{H}$ & 1.26685469420438 & 8.73160215397555 & 13.01662309092581 \\
\hline $\mathrm{C}$ & -2.17734828032824 & 7.23304238867029 & 12.24516743142294 \\
\hline $\mathrm{H}$ & -2.05771961957982 & 8.31103964014575 & 12.10544002136137 \\
\hline $\mathrm{C}$ & -1.94567210330997 & 6.52924802292755 & 10.89893406615332 \\
\hline $\mathrm{H}$ & -0.95793018251610 & 6.77295571365960 & 10.49656321334146 \\
\hline $\mathrm{H}$ & -1.97172074767513 & 5.44143348677777 & 11.06484195896127 \\
\hline $\mathrm{C}$ & -3.01334333065235 & 6.92858422041731 & 9.87405724776714 \\
\hline $\mathrm{H}$ & -2.90960031793977 & 8.00166687825122 & 9.65509940816219 \\
\hline $\mathrm{H}$ & -2.83682424885517 & 6.39287782054487 & 8.93241286923794 \\
\hline $\mathrm{C}$ & -4.42887157543692 & 6.65740272779979 & 10.38720038125901 \\
\hline $\mathrm{H}$ & -4.56448512870584 & 5.57228315898497 & 10.51203799517670 \\
\hline $\mathrm{H}$ & -5.17195325670334 & 6.99096912722174 & 9.65091993740765 \\
\hline $\mathrm{C}$ & -4.65875351769571 & 7.34867590589332 & 11.73231412882343 \\
\hline $\mathrm{H}$ & -5.65761817042304 & 7.10743294076382 & 12.11925935775186 \\
\hline $\mathrm{H}$ & -4.62071078656334 & 8.43788949685734 & 11.59074339367194 \\
\hline $\mathrm{C}$ & -3.59583166843600 & 6.93540193178864 & 12.75205508989562 \\
\hline $\mathrm{H}$ & -3.65783344703204 & 5.85201905650521 & 12.93628868478959 \\
\hline $\mathrm{H}$ & -3.75875521128873 & 7.42672637730299 & 13.71461125956560 \\
\hline $\mathrm{C}$ & -0.69820560031056 & 7.91302991848192 & 14.84778918850777 \\
\hline $\mathrm{C}$ & -0.01583245887517 & 7.36345417785725 & 15.95318937215797 \\
\hline $\mathrm{H}$ & 0.05402250767801 & 6.27769964861335 & 16.01392683006772 \\
\hline $\mathrm{C}$ & 0.58096591865722 & 8.15816866525114 & 16.92655248705582 \\
\hline $\mathrm{H}$ & 1.10688812800030 & 7.69455404356942 & 17.75830413948150 \\
\hline $\mathrm{C}$ & 0.50574595901648 & 9.54663563709705 & 16.81903942640452 \\
\hline $\mathrm{H}$ & 0.98673893979522 & 10.18856300836721 & 17.55354664351425 \\
\hline
\end{tabular}




$\begin{array}{cccc}\mathrm{C} & -0.22978336703528 & 10.10609507409644 & 15.77819446120007 \\ \mathrm{H} & -0.35105221308874 & 11.18606427619354 & 15.72161147203778 \\ \mathrm{C} & -0.87295199447089 & 9.31698316341959 & 14.80770916250366 \\ \mathrm{C} & -1.71273726750354 & 10.04559813767271 & 13.81069119123621 \\ \mathrm{C} & -1.05495579244179 & 10.70659861236970 & 12.76764239629058 \\ \mathrm{H} & 0.02981416163283 & 10.66142831101739 & 12.74221186374027 \\ \mathrm{C} & -1.74129139211805 & 11.36409367616312 & 11.74752471804375 \\ \mathrm{H} & -1.19670388287322 & 11.83924513543183 & 10.93547486118042 \\ \mathrm{C} & -3.13266180272910 & 11.39280229043614 & 11.79507691349177 \\ \mathrm{H} & -3.70179248079039 & 11.89542938590822 & 11.01564017615799 \\ \mathrm{C} & -3.80970033376910 & 10.80605179381826 & 12.86122492650944 \\ \mathrm{H} & -4.88932648925290 & 10.88991146092145 & 12.89216792484131 \\ \mathrm{C} & -3.13416478269627 & 10.12598202399801 & 13.89681361702276 \\ \mathrm{C} & -3.51581921223621 & 9.78943435172164 & 16.32000015297996 \\ \mathrm{H} & -2.44752004164669 & 9.68506309806059 & 16.48962125180910 \\ \mathrm{H} & -4.03543810942248 & 9.07240292125379 & 16.96242588409471 \\ \mathrm{H} & -3.82285275856521 & 10.80847802820524 & 16.61586287236721 \\ \mathrm{C} & -5.31246361763565 & 9.42445209405579 & 14.76860291213101 \\ \mathrm{H} & -5.57184086476222 & 9.03051201673125 & 13.78428103723431 \\ \mathrm{H} & -5.82025043232391 & 10.39696383649424 & 14.90304175682868 \\ \mathrm{H} & -5.69810394284399 & 8.73526126071524 & 15.52642770829956\end{array}$

(Pd(0)-DavePhos complex with Pd-aryl binding and DME bound)

Final Gibbs free enthalpy $\left(\Delta \mathrm{G}=\Delta \mathrm{H}-\mathrm{T}^{*} \Delta \mathrm{S}\right)=-1843.18123072 E_{\mathrm{h}}$

$\begin{array}{cccc}\text { Pd } & -0.49898057438553 & 4.84216373173623 & 15.24805383313983 \\ \mathrm{P} & -0.75695100575569 & 6.60348291126190 & 13.90715102064526 \\ \mathrm{~N} & -1.81225917632911 & 6.16944789276283 & 17.68052282322437 \\ \mathrm{C} & 0.67912579922467 & 6.80311381679696 & 12.72227831780308 \\ \mathrm{H} & 0.63694379893260 & 5.87213075788711 & 12.13750102453365 \\ \mathrm{C} & 1.98872842865114 & 6.78692601763896 & 13.51994957598154 \\ \mathrm{H} & 2.02903213973271 & 7.68810533852593 & 14.14702957141644 \\ \mathrm{H} & 1.98790433063319 & 5.93201635981447 & 14.20766816103804 \\ \mathrm{C} & 3.20939478427983 & 6.76481174188897 & 12.60038756086703 \\ \mathrm{H} & 4.12928801551915 & 6.79047612301871 & 13.19961495029086 \\ \mathrm{H} & 3.22462787912899 & 5.81957040101501 & 12.03603829558860 \\ \mathrm{C} & 3.18253643252699 & 7.93691678971640 & 11.61584690658463 \\ \mathrm{H} & 4.04053542615034 & 7.88598600069965 & 10.93308651262914 \\ \mathrm{H} & 3.27678582505517 & 8.87760509290722 & 12.17949470907541 \\ \mathrm{C} & 1.87312915107917 & 7.96359017443237 & 10.82280795716150 \\ \mathrm{H} & 1.84971099799483 & 8.83643842041242 & 10.15647590075237 \\ \mathrm{H} & 1.81975589556616 & 7.06996440580987 & 10.18180297414185 \\ \mathrm{C} & 0.65244142372155 & 7.98597950439205 & 11.75215656078312 \\ \mathrm{H} & -0.26673147974492 & 7.98229547518293 & 11.15503005063562 \\ \mathrm{H} & 0.65828665873489 & 8.92201983652608 & 12.32575807607282 \\ \mathrm{C} & -2.25733994316914 & 6.84689099647480 & 12.84069619419837\end{array}$




\begin{tabular}{|c|c|c|c|}
\hline & -2.19722461954670 & & \\
\hline & -2.32277264285822 & & \\
\hline & -1.45720890909548 & & 1.12368331280799 \\
\hline & -2.25755113450986 & & \\
\hline & -3.61363700899361 & & \\
\hline & -3.61236443969913 & & \\
\hline & -3.64415680678008 & & \\
\hline & -4.85412374 & 5.7774 & \\
\hline & -4.90830776 & & \\
\hline & -5.76422737 & & 30797 \\
\hline & -4.79 & & \\
\hline & $-5.6^{\circ}$ & & \\
\hline & -4.8 & & \\
\hline & -3.51 & & \\
\hline & -3.50621981 & & \\
\hline & -3.4 & & \\
\hline & -0.6 & & \\
\hline & -1.0 & & \\
\hline & -1.5 & & \\
\hline & -0.8 & & \\
\hline & -1.1 & & \\
\hline & -0.1 & & \\
\hline & 0.05 & & \\
\hline & 0.23 & & \\
\hline & 0.73 & & \\
\hline & -0.02 & & 025 \\
\hline & & & \\
\hline & & & \\
\hline & 2.45 & & \\
\hline & & & \\
\hline & & & \\
\hline & & & \\
\hline & & & \\
\hline & 0.08 & & \\
\hline & -0.5 & & \\
\hline & -0.4 & 5.903 & 48308 \\
\hline & -2.32 & & \\
\hline & $-1.5^{\circ}$ & 8.1798 & 34784 \\
\hline & -3.19 & & \\
\hline & & & \\
\hline & 63034 & & 80500 \\
\hline & -2.44 & & \\
\hline & -3.405674689 & 5.39378 & 16.52332311496608 \\
\hline & -3.56969366543341 & & \\
\hline $\mathrm{O}$ & -1.83427749197970 & 2.48466079097153 & 15.24207111128268 \\
\hline & -1.07469939118832 & 1.56039315570731 & 14.4703516633825 \\
\hline
\end{tabular}




$\begin{array}{llcc}\mathrm{H} & -1.47135829046750 & 0.53840821874301 & 14.60244069195282 \\ \mathrm{H} & -1.15219750202297 & 1.81402819765787 & 13.40139340311011 \\ \mathrm{C} & 0.36943458786782 & 1.59238782902906 & 14.92707922273687 \\ \mathrm{H} & 0.89971403726356 & 0.73279296075734 & 14.48604532108079 \\ \mathrm{H} & 0.40619931053366 & 1.50830047111896 & 16.01956631455293 \\ \mathrm{O} & 1.05226291989124 & 2.80376549235969 & 14.61460152944622 \\ \mathrm{C} & -3.13672265945931 & 2.67378132054060 & 14.70290543010946 \\ \mathrm{H} & -3.08350497909978 & 3.07694438646340 & 13.68071873450464 \\ \mathrm{H} & -3.69961199329904 & 1.72655557172115 & 14.68968084988436 \\ \mathrm{H} & -3.64924053430987 & 3.39573041699232 & 15.34093750835503 \\ \mathrm{C} & 1.39792466224908 & 2.93414101538081 & 13.23888700052645 \\ \mathrm{H} & 0.51443991780812 & 3.06378615149562 & 12.59949744015538 \\ \mathrm{H} & 2.01489662955806 & 3.83187010508185 & 13.16012707310753 \\ \mathrm{H} & 1.97142484154706 & 2.05741709916472 & 12.89596760791511\end{array}$

(Pd(0)-bisDavePhos complex without Pd-aryl binding)

Final Gibbs free enthalpy $\left(\Delta \mathrm{G}=\Delta \mathrm{H}-\mathrm{T}^{*} \Delta \mathrm{S}\right)=-2942.27140296 E_{\mathrm{h}}$

$\begin{array}{cccc}\text { Pd } & 0.53000214696895 & 4.79291317219996 & 13.90633042637720 \\ \mathrm{P} & -0.13275988471627 & 6.82875717594384 & 13.12437171264216 \\ \mathrm{~N} & -3.21368388234795 & 7.84548849962247 & 16.00646194352796 \\ \mathrm{C} & 0.84840725053803 & 7.67236209347814 & 11.78142193143441 \\ \mathrm{H} & 0.65244111312638 & 7.03506302431706 & 10.90787141851284 \\ \mathrm{C} & 2.35433021644444 & 7.58582691523049 & 12.05935040006736 \\ \mathrm{H} & 2.59484763734309 & 8.15911245235322 & 12.96415306957921 \\ \mathrm{H} & 2.62276243670349 & 6.54214653159937 & 12.26518422508454 \\ \mathrm{C} & 3.16256253806668 & 8.14459928444766 & 10.88414007161857 \\ \mathrm{H} & 4.23534693124603 & 8.10077345497258 & 11.11392150202035 \\ \mathrm{H} & 2.99563300473873 & 7.51173687047316 & 9.99948547443504 \\ \mathrm{C} & 2.74815475335143 & 9.58257768496579 & 10.55605154462123 \\ \mathrm{H} & 3.31348768910367 & 9.95478638908205 & 9.69161748909133 \\ \mathrm{H} & 3.00002983702828 & 10.23320052567849 & 11.40777570039931 \\ \mathrm{C} & 1.24372634866352 & 9.67720875630288 & 10.28712387115232 \\ \mathrm{H} & 0.95273793048821 & 10.71992614515196 & 10.10507306539264 \\ \mathrm{H} & 1.00261388010922 & 9.11608402823715 & 9.37183700173011 \\ \mathrm{C} & 0.42868607242949 & 9.10611696480828 & 11.45296119650660 \\ \mathrm{H} & -0.64164346296754 & 9.14846930639827 & 11.22087837280002 \\ \mathrm{H} & 0.58711073874256 & 9.72866288636608 & 12.34370835254222 \\ \mathrm{C} & -1.87920312187520 & 6.76998283909019 & 12.49130533841625 \\ \mathrm{H} & -2.33400833565644 & 7.76408129528815 & 12.52642395527209 \\ \mathrm{C} & -1.90874489273523 & 6.26460234359091 & 11.03936157098607 \\ \mathrm{H} & -1.39257909422664 & 6.96304565268683 & 10.37187933085640 \\ \mathrm{H} & -1.37107127890542 & 5.30518555599631 & 10.98856532461146 \\ \mathrm{C} & -3.34802301597061 & 6.07139315619130 & 10.54538722435859 \\ \mathrm{H} & -3.84505085824948 & 7.05223177062468 & 10.51240749569772 \\ \mathrm{H} & -3.33458484739981 & 5.68298490708559 & 9.51860072808843\end{array}$




\begin{tabular}{|c|c|c|c|}
\hline & -4.14826161138632 & & \\
\hline & -3.70949302578352 & & 11.42324677747900 \\
\hline & 5.18267020193568 & 05517053212799 & \\
\hline & & & \\
\hline & 4.65876983799515 & & \\
\hline & -4.62653172707690 & & \\
\hline & -2.67363654969669 & & \\
\hline & -2.15636892838125 & & \\
\hline & -2.6432 & & \\
\hline & 0.06217 & & \\
\hline & 1.27 & & \\
\hline & 597 & & \\
\hline & 1.76 & & \\
\hline & 2.70 & & \\
\hline & 1.04 & & \\
\hline & 1.41399 & & \\
\hline & -0.1905 & & \\
\hline & -0.7 & & \\
\hline & -0.71 & & \\
\hline & -2.10 & & \\
\hline & -2.2 & & \\
\hline & -1.3 & & \\
\hline & -3.48 & & \\
\hline & -3.5 & & \\
\hline & -4.6 & & \\
\hline & -5.5 & 038 & \\
\hline & -4.5 & & \\
\hline & -5.4 & & \\
\hline & -3.2 & & \\
\hline & -2.2 & & \\
\hline & -2.1 & & \\
\hline & $-1.2^{\prime}$ & & \\
\hline & -2.6 & & \\
\hline & -4.41 & & \\
\hline & -4.85 & & \\
\hline & -5.15 & & \\
\hline & -4.12 & & \\
\hline & 0.653 & 2.82 & 15.0 \\
\hline & $2.30^{\circ}$ & & \\
\hline $\mathrm{C}$ & 0.08 & & \\
\hline & 0381658368 & & \\
\hline & 2.879 & & \\
\hline & $3.022^{\prime}$ & 693491466 & \\
\hline & 2.28984343843640 & & \\
\hline & -0.24159452359125 & 2.08740620473672 & 17.20732727991465 \\
\hline & 1.22847400552317 & 3.59042024212513 & 17.67099186754299 \\
\hline
\end{tabular}




\begin{tabular}{|c|c|c|c|}
\hline & & & \\
\hline & -0.11086969175521 & & \\
\hline & & & \\
\hline & 45843575046380 & 1.27857048094109 & \\
\hline & & & \\
\hline & 44193702765556 & .35872215142875 & \\
\hline & & & \\
\hline & & & \\
\hline & & & \\
\hline & .05125245271442 & & 21 \\
\hline & & & \\
\hline & 0.73 & & \\
\hline & -1.58 & & 992 \\
\hline & & & \\
\hline & -1.8 & & 2001 \\
\hline $\mathrm{H}$ & 0.37 & & \\
\hline & 66 & & 2061 \\
\hline & & & 6678 \\
\hline & & & 8380 \\
\hline & & & \\
\hline $\mathrm{H}$ & 5.04 & & \\
\hline & 4.42 & -0.0 & \\
\hline $\mathrm{H}$ & & & \\
\hline & 4.28 & & \\
\hline & & & \\
\hline & 1.56 & & \\
\hline C & & & 4224 \\
\hline & -2.4 & & 0561 \\
\hline $\mathrm{H}$ & & & \\
\hline & -0.2 & & \\
\hline & -1.0 & & \\
\hline H & -1.9 & & 0042 \\
\hline$y$ & -0.9 & & \\
\hline $\mathrm{C}$ & -2.7 & & 7494 \\
\hline & 5.45 & -0.4 & \\
\hline $\mathrm{H}$ & 3.90214402 & -0.73 & 9184 \\
\hline $\mathrm{H}$ & -0.13338691 & & \\
\hline $\mathrm{H}$ & -0.8105733 & & \\
\hline H & -1.3625857 & & 37907 \\
\hline H & -0.0 & & 0238 \\
\hline$C$ & -1.30 & -0.6 & 3096 \\
\hline $\mathrm{N}$ & $-3.4515211<$ & & \\
\hline $\mathrm{C}$ & -3.126629274 & 0.8685125 & 17.82618925109939 \\
\hline & -0.74684482 & & \\
\hline- & -2.41221740730272 & & 18.69382149367746 \\
\hline & -3.83809577632805 & 1.65963868000419 & 14.29985649844505 \\
\hline
\end{tabular}




$\begin{array}{llrl}\mathrm{C} & -4.40523673115853 & 2.85179529913434 & 16.30484740026727 \\ \mathrm{H} & -4.00720848591487 & 1.37296101678826 & 18.20523124055128 \\ \mathrm{H} & -2.74294438402633 & -0.04750443867494 & 19.72589559431893 \\ \mathrm{H} & -3.19836402838713 & 0.90281410060645 & 13.85851581576687 \\ \mathrm{H} & -3.76052785846212 & 2.56929231222995 & 13.69425405570990 \\ \mathrm{H} & -4.88072421009220 & 1.29931842797433 & 14.25923717493591 \\ \mathrm{H} & -3.99158630289218 & 3.28555474191451 & 17.21658762860092 \\ \mathrm{H} & -5.36375171065085 & 2.36046264146924 & 16.54941736569685 \\ \mathrm{H} & -4.61510215413226 & 3.66650754974812 & 15.60427093267975\end{array}$

(Pd(0)-bisDavePhos complex with Pd-aryl binding)

Final Gibbs free enthalpy $\left(\Delta \mathrm{G}=\Delta \mathrm{H}-\mathrm{T}^{*} \Delta \mathrm{S}\right)=-2942.30397556 E_{\mathrm{h}}$

$\begin{array}{cccc}\text { Pd } & 0.04344939064793 & 4.75271409387444 & 13.90033075317371 \\ \text { P } & -0.11100470277587 & 6.99413839125832 & 13.38625727807970 \\ \text { N } & 2.87003942423988 & 5.66152416149965 & 11.99727867099999 \\ \text { C } & -1.07249356681532 & 7.18445846623750 & 11.78495226942506 \\ \text { H } & -1.68656918008667 & 6.27739935028525 & 11.82843152099651 \\ \text { C } & -0.11608235151652 & 7.02792792872194 & 10.59626207724242 \\ \text { H } & 0.52078379436552 & 7.92053416984999 & 10.52790592804564 \\ \text { H } & 0.54929636452986 & 6.17881036098025 & 10.77892750112886 \\ \mathrm{C} & -0.88188528628252 & 6.84432974129055 & 9.28384716488370 \\ \mathrm{H} & -0.17742179941905 & 6.78295005712601 & 8.44389949719172 \\ \mathrm{H} & -1.42076853158882 & 5.88601180741770 & 9.31572152942320 \\ \mathrm{C} & -1.88572925117386 & 7.97733402494770 & 9.05587593426779 \\ \mathrm{H} & -2.45997163402406 & 7.79968686062036 & 8.13731224536035 \\ \mathrm{H} & -1.33472676770079 & 8.91924943576689 & 8.91230960514438 \\ \mathrm{C} & -2.82987592053034 & 8.13281475593537 & 10.25139395241265 \\ \mathrm{H} & -3.51602153580201 & 8.97355036333195 & 10.08544356980034 \\ \mathrm{H} & -3.44776111228266 & 7.22869326533865 & 10.34508218722380 \\ \mathrm{C} & -2.04655612176210 & 8.34929354193573 & 11.55436277408294 \\ \mathrm{H} & -2.74120947026464 & 8.45538858948975 & 12.39444354530587 \\ \mathrm{H} & -1.50538888189431 & 9.29779205095763 & 11.46119126012346 \\ \mathrm{C} & -1.17678497317083 & 7.79562220205160 & 14.68855325729705 \\ \mathrm{H} & -1.44286865369751 & 8.80790700544667 & 14.35356495049719 \\ \mathrm{C} & -2.44774593331664 & 6.96470660299457 & 14.87356889129963 \\ \mathrm{H} & -3.01151240875626 & 6.92560807153194 & 13.93933485758044 \\ \mathrm{H} & -2.14431262615298 & 5.92781375732275 & 15.07594317679713 \\ \mathrm{C} & -3.32801022671234 & 7.49516857232940 & 16.00487715884363 \\ \mathrm{H} & -3.71089822432123 & 8.49166787138598 & 15.73620994061549 \\ \mathrm{H} & -4.20095547877481 & 6.84097654648122 & 16.12650694753778 \\ \mathrm{C} & -2.54522303788700 & 7.59145386897141 & 17.31579272946395 \\ \mathrm{H} & -2.25892275607841 & 6.57813635802603 & 17.63125433897020 \\ \mathrm{H} & -3.17494137552929 & 8.00820414922500 & 18.11278652996395 \\ \mathrm{C} & -1.27985667775104 & 8.43583174675484 & 17.14045433417926 \\ \mathrm{H} & -0.70617760230415 & 8.46108721167054 & 18.07640384968679\end{array}$




\begin{tabular}{|c|c|c|c|}
\hline & -1.56726897522474 & & \\
\hline & -0.39908327988478 & & \\
\hline & -0.02598283663284 & & \\
\hline & 0.48150669721868 & & \\
\hline & 1.21820052592579 & & \\
\hline & & & \\
\hline & -0.16854273229245 & & \\
\hline & 1.76887361921489 & 10.64 & \\
\hline & & & \\
\hline & 3.08550512594 & 10.4 & 13.3 \\
\hline & & & \\
\hline & $3.45^{\circ}$ & & \\
\hline & & & \\
\hline & 2.5496 & & \\
\hline & 3.08311124308277 & & \\
\hline & 3.51 & & \\
\hline & 3.34 & & \\
\hline & & & \\
\hline & 4.43 & & $17 .($ \\
\hline & 4.31 & & \\
\hline & 4.79 & & \\
\hline & 3.89 & & \\
\hline & 4.06 & & \\
\hline & 3.27 & & \\
\hline & & & \\
\hline & 3.71 & & \\
\hline & & & \\
\hline & 4.6 & & \\
\hline & 2.59 & & \\
\hline & & & \\
\hline & & & \\
\hline & 2.06 & & \\
\hline & 0.03 & & \\
\hline & & & \\
\hline & 0.16 & & \\
\hline & -1.28 & & 129 \\
\hline & $2.26^{\prime}$ & & \\
\hline 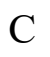 & 1.39 & & \\
\hline C & 2.34 & 0.92 & \\
\hline & 0.38 & & 17.329 \\
\hline$C$ & 1.305 & 4.04 & 17.058 \\
\hline $\mathrm{C}$ & -1.16 & & 17.3 \\
\hline $\mathrm{C}$ & -1.03057161699432 & 0.23315381 & 15.75994194720730 \\
\hline & -2.57807534249458 & & \\
\hline & 0.73378719079630 & 0.15465735567451 & 13.42966506901331 \\
\hline & 0.88857736481872 & 1.64301152332996 & 12.51165055248076 \\
\hline
\end{tabular}




\begin{tabular}{|c|c|c|c|}
\hline & 2.72767872516692 & & \\
\hline & & & \\
\hline & .50273767280822 & & \\
\hline & 76037411940696 & & \\
\hline & & & \\
\hline & & & \\
\hline & 39677728889068 & & \\
\hline & .06975343009598 & & 055 \\
\hline & 152 & & \\
\hline & -1.96537745 & & 17. \\
\hline & & & \\
\hline & 482 & & 7200 \\
\hline & & & \\
\hline & 38 & & 9512 \\
\hline & 2.542489250 & & \\
\hline $\mathrm{H}$ & & & \\
\hline & 3.508043 & & \\
\hline & & & \\
\hline & 65 & & \\
\hline $\mathrm{H}$ & & & \\
\hline & 00 & & \\
\hline $\mathrm{C}$ & 0.06389 & & \\
\hline $\mathrm{H}$ & -2.0268249 & & \\
\hline & -0.8901997 & & \\
\hline $\mathrm{H}$ & -1.752 & & \\
\hline & -3.271 & & 664 \\
\hline $\mathrm{H}$ & -4.542 & & \\
\hline & -4.055 & & 025 \\
\hline C & -2.56734 & & 7529 \\
\hline & 4.483194 & & \\
\hline $\mathrm{H}$ & & & \\
\hline $\mathrm{H}$ & -0.16 & & \\
\hline $\mathrm{H}$ & 0.136 & & \\
\hline $\mathrm{H}$ & -4.04397 & & 620 \\
\hline & -4.39728 & & \\
\hline $\mathrm{C}$ & -4.62263 & & 952 \\
\hline & -1.56023 & & \\
\hline $\mathrm{C}$ & -3.14591497 & & 9623 \\
\hline $\mathrm{H}$ & -5.39675228 & & 421509 \\
\hline & -4.15860048 & & 31033 \\
\hline & -1.94662856 & 1962 & 517149577 \\
\hline $\mathrm{C}$ & -0.74263548 & & 371367244 \\
\hline & -2.81285811130332 & & 6257607852 \\
\hline & & & \\
\hline & -2.48788640257944 & 0.52830277994562 & 12.23308668508372 \\
\hline & -1.04404245289211 & 0.46261316488781 & 11.20821351132664 \\
\hline
\end{tabular}



Н -2.58544555690636
1.06078696332919
10.53250738630693
H -0.39335029308367
4.00861527151445
11.34423025758519
H -1.26176690496085
3.29114097735089
9.93358048231532
H 0.13075898665587
2.45819471736179
10.65309347789173

\section{5 (Pd(0)-DavePhos complex with Pd-aryl binding with 4-methoxybiphenyl bound)}

Final Gibbs free enthalpy $\left(\Delta \mathrm{G}=\Delta \mathrm{H}-\mathrm{T}^{*} \Delta \mathrm{S}\right)=-2112.00651630 E_{\mathrm{h}}$

\begin{tabular}{|c|c|c|c|}
\hline & & & \\
\hline$P$ & -0.56164446819281 & & \\
\hline & & & \\
\hline & 0.13731764930396 & 1747 & \\
\hline & & & \\
\hline & -2.56024666816338 & 1.35588195592690 & \\
\hline$C^{\prime}$ & & & \\
\hline & 533 & -0.05 & \\
\hline 1 & & 0.62 & \\
\hline & 31 & 1.27 & 703 \\
\hline $\mathrm{C}$ & & 2.35 & \\
\hline $\mathrm{C}$ & 1.51 & 1.75 & \\
\hline & 1.21 & 4.14 & 17.0 \\
\hline $\mathrm{H}$ & & & \\
\hline & -2.7 & 3.0 & 17. \\
\hline $\mathrm{C}$ & -3.5 & & \\
\hline & -2.5 & -0.8 & 17. \\
\hline $\mathrm{H}$ & -0.9 & & \\
\hline $\mathrm{I}$ & -0.5 & -0.0 & 17. \\
\hline $\mathrm{H}$ & & & \\
\hline $\mathrm{H}$ & & & \\
\hline $\mathrm{C}$ & -0.4 & 0.5 & 11.8 \\
\hline $\mathrm{C}$ & & & \\
\hline & $0.8^{-}$ & & \\
\hline $\mathrm{H}$ & 2.08 & & \\
\hline & 1.27 & & \\
\hline $\mathrm{C}$ & 2.47 & & \\
\hline $\mathrm{C}$ & 2.18 & & \\
\hline $\mathrm{C}$ & 0.73 & & \\
\hline $\mathrm{H}$ & -4.0 & 26003 & 7696 \\
\hline $\mathrm{H}$ & -4.3 & & 17.3 \\
\hline $\mathrm{C}$ & -3.18 & 8333050 & 7167 \\
\hline $\mathrm{H}$ & -2.21 & -1.9 & \\
\hline $\mathrm{H}$ & $-3.28942620^{\prime}$ & 02510846 & 16.3316 \\
\hline & -0.08516797 & -0.407 & \\
\hline $\mathrm{H}$ & -1.28124045115573 & 0.45513589164763 & 11.14644552200510 \\
\hline & 0.677031997 & 1.39655766381874 & 11.21212042232422 \\
\hline & 2.59781673373723 & 2.25148457340189 & 11.76075838973790 \\
\hline
\end{tabular}




\begin{tabular}{|c|c|c|c|}
\hline & & & \\
\hline & 2.97647009752467 & & \\
\hline & 2.79917746321609 & 3.29660987550085 & 18.66711295236257 \\
\hline & & & \\
\hline & 35475322367360 & 02 & \\
\hline & & & \\
\hline & .05547931135706 & -0.83979704522423 & 58108 \\
\hline & .46204177454313 & -0.27121231294471 & 19.200 \\
\hline & 28507853818609 & & 309 \\
\hline & & & \\
\hline & 57567285738 & & 344 \\
\hline & & 46 & \\
\hline & & & \\
\hline & 10166 & 477 & 594 \\
\hline & & & \\
\hline & & & \\
\hline & & & \\
\hline & 293 & & 886 \\
\hline & & & \\
\hline $\mathrm{H}$ & 26 & & 7210 \\
\hline & 0.83 & & \\
\hline $\mathrm{H}$ & & & 001 \\
\hline & -1.3 & & 690 \\
\hline & -2.2 & & 462 \\
\hline & -1.5 & & 756 \\
\hline & 0.21 & & 660 \\
\hline & & & 1569 \\
\hline & & & \\
\hline & & & 124 \\
\hline & -3.5 & & \\
\hline & & & 583 \\
\hline & -4.6 & & 167 \\
\hline & -4.1 & & 300 \\
\hline & -3.0 & & 6227 \\
\hline & -4.87 & & \\
\hline & 639540 & & 3355 \\
\hline & $-3.9^{\prime}$ & & \\
\hline$H$ & -5.28 & & 9009 \\
\hline & -3.2 & & 2165 \\
\hline & -3.8 & & 5658 \\
\hline & -2.08 & & \\
\hline & -3.42293210667592 & 4.5073828447 & 71798 \\
\hline & & & \\
\hline $\mathrm{C}$ & -1.57148730355912 & & 12.57520720406256 \\
\hline & -1.67051589408896 & 7.19474374314146 & 14.37536219419816 \\
\hline & -2.22039975994808 & 5.10507939007690 & 11.74640912714692 \\
\hline
\end{tabular}




$\begin{array}{cccc}\mathrm{H} & -3.96990740364504 & 3.82937263441664 & 11.52229455980426 \\ \mathrm{H} & -0.68954175606716 & 6.53474022475337 & 12.20400616044842 \\ \mathrm{O} & -1.62438682185023 & 4.86301828071125 & 10.53426334045117 \\ \mathrm{C} & -2.21290909196344 & 3.87449058908079 & 9.68717082449149 \\ \mathrm{H} & -2.27175044388292 & 2.90173138502921 & 10.19499771225175 \\ \mathrm{H} & -3.21637237041082 & 4.17499473312277 & 9.35802629935844 \\ \mathrm{H} & -1.55312094358974 & 3.79467493063480 & 8.82054992262722\end{array}$

6Cl (Pd(0)-DavePhos complex with Pd-aryl binding with 4-methoxychlorobenzene bound)

Final Gibbs free enthalpy $\left(\Delta \mathrm{G}=\Delta \mathrm{H}-\mathrm{T}^{*} \Delta \mathrm{S}\right)=-2340.74658017 E_{\mathrm{h}}$

$\begin{array}{cccc}\mathrm{Pd} & -0.87692651781758 & 4.46828449792228 & 14.55297271829404 \\ \mathrm{P} & -0.77778322766487 & 6.61236160633729 & 13.74737709871685 \\ \mathrm{~N} & -2.41553956028225 & 6.15279700090495 & 16.79198427089016 \\ \mathrm{C} & 0.75839516830131 & 6.69667956665232 & 12.69599166249681 \\ \mathrm{H} & 0.58129399862370 & 5.90201886706855 & 11.95719076989690 \\ \mathrm{C} & 1.96688439120081 & 6.26667807897733 & 13.53993548297663 \\ \mathrm{H} & 2.11779249682393 & 7.00160370827355 & 14.34239187870125 \\ \mathrm{H} & 1.74146227865626 & 5.30775970714414 & 14.02321188054431 \\ \mathrm{C} & 3.23658292333665 & 6.17273814995635 & 12.69255038311863 \\ \mathrm{H} & 4.09023875933637 & 5.90754637984088 & 13.33051043724292 \\ \mathrm{H} & 3.11802577796039 & 5.36058142677074 & 11.96205169518899 \\ \mathrm{C} & 3.51303662797078 & 7.48186884782578 & 11.94749236995293 \\ \mathrm{H} & 4.40622657010488 & 7.38118164438393 & 11.31711209200402 \\ \mathrm{H} & 3.72404428688317 & 8.27549919336286 & 12.67941799624038 \\ \mathrm{C} & 2.30703809094369 & 7.89521856017343 & 11.09909527272153 \\ \mathrm{H} & 2.50148371646069 & 8.85227369968380 & 10.59681994217055 \\ \mathrm{H} & 2.14734649208870 & 7.14596071287945 & 10.30936448334751 \\ \mathrm{C} & 1.03735997483767 & 8.00464574802749 & 11.95240651451274 \\ \mathrm{H} & 0.18566184060891 & 8.27836921918438 & 11.31835812418923 \\ \mathrm{H} & 1.16932670058241 & 8.81333044749348 & 12.68295994437315 \\ \mathrm{C} & -2.16385081227273 & 7.13165999834900 & 12.63228801474524 \\ \mathrm{H} & -1.97333684811998 & 8.14536512722675 & 12.25658132967052 \\ \mathrm{C} & -2.27362616372148 & 6.18918566599400 & 11.42561947574231 \\ \mathrm{H} & -1.35773406406273 & 6.22714771695780 & 10.82640880015367 \\ \mathrm{H} & -2.37022603351102 & 5.15872959116504 & 11.79150723896302 \\ \mathrm{C} & -3.48200158179198 & 6.54171935731238 & 10.55080478226965 \\ \mathrm{H} & -3.32694296355694 & 7.53572792999290 & 10.10432203709320 \\ \mathrm{H} & -3.55654494182942 & 5.82922042409880 & 9.71923591495437 \\ \mathrm{C} & -4.78038154940026 & 6.55277129898807 & 11.36132913738581 \\ \mathrm{H} & -4.98467767931727 & 5.53480039942882 & 11.72591683730297 \\ \mathrm{H} & -5.62566036429093 & 6.83940483162696 & 10.72228554878719 \\ \mathrm{C} & -4.67347600859562 & 7.49690087249647 & 12.56172899403267 \\ \mathrm{H} & -5.59343894901302 & 7.45872671398518 & 13.16003823836975 \\ \mathrm{H} & -4.56858748902980 & 8.53212460749762 & 12.20352115286882 \\ \mathrm{C} & -3.47061459373209 & 7.13820626732989 & 13.43740677380994\end{array}$




\begin{tabular}{|c|c|c|c|}
\hline & -3.61132481264928 & & \\
\hline & & & \\
\hline & .57130935598215 & & .88278667814720 \\
\hline & & 9.36788469316632 & \\
\hline & -1.09224761428135 & & \\
\hline & .45354925955355 & & 15.22527702568036 \\
\hline & -0.59875162000595 & 11.47660978857862 & \\
\hline & 0.03558259498034 & & \\
\hline & & & 0866 \\
\hline & 480846 & & 274 \\
\hline & 0.53 & & \\
\hline & -0.13 & 120 & 0172 \\
\hline & & & \\
\hline & 1.287 & & \\
\hline & & & \\
\hline & 1.49 & & \\
\hline & 2.49 & & \\
\hline & 0.40 & & \\
\hline & 0.53 & & 18.1 \\
\hline & -0.8 & & 693 \\
\hline & -1.72 & & 7372 \\
\hline & & & \\
\hline & -2.86 & & \\
\hline & -2.08 & & \\
\hline $\mathrm{H}$ & -3.1 & & \\
\hline & -3.7 & & 17. \\
\hline & -3.4 & & 660 \\
\hline & -3.7 & & \\
\hline & -3.0 & 681 & \\
\hline & -4.3 & & \\
\hline & & & \\
\hline & -0.74 & & \\
\hline & -0.97 & & 770 \\
\hline & 0.57 & & \\
\hline & 0.13 & & \\
\hline & -1.97 & 8835 & 507 \\
\hline & 1.640 & & \\
\hline & 0.73 & & 15.8 \\
\hline $\mathrm{C}$ & 1.415 & & \\
\hline $\mathrm{H}$ & & & 11.1707083 \\
\hline H & 2.638 & 37208 & \\
\hline $\mathrm{O}$ & 2.41 & & \\
\hline $\mathrm{C}$ & 3.749 & 2.410 & 12.09349737 \\
\hline & & & \\
\hline & 4.37319040299523 & 2.60594267965979 & 11.21790316864612 \\
\hline & 4.08062701668357 & 3.04589924350188 & 12.92646990067286 \\
\hline
\end{tabular}


6F (Pd(0)-DavePhos complex with Pd-aryl binding with 4-methoxyfluorobenzene bound)

Final Gibbs free enthalpy $\left(\Delta \mathrm{G}=\Delta \mathrm{H}-\mathrm{T}^{*} \Delta \mathrm{S}\right)=-1980.33184320 E_{\mathrm{h}}$

\begin{tabular}{|c|c|c|c|}
\hline & -0.86621786366489 & 4.47634623054873 & 14.53470767175026 \\
\hline $\mathrm{P}$ & -0.79338255180341 & 6.62096841044020 & 13.72089031180617 \\
\hline $\mathrm{N}$ & -2.38888251941127 & 6.13646444555315 & 16.79011286743183 \\
\hline $\mathrm{C}$ & 0.73377090436598 & 6.71006187249252 & 12.65600266797876 \\
\hline $\mathrm{H}$ & 0.55103926449626 & 5.91665574183374 & 11.91734971815377 \\
\hline $\mathrm{C}$ & 1.94947342674013 & 6.27823972785232 & 13.48873722077447 \\
\hline $\mathrm{H}$ & 2.10982939155239 & 7.01406725427538 & 14.28854322058449 \\
\hline $\mathrm{H}$ & 1.72479855417073 & 5.32108294499954 & 13.97610199915672 \\
\hline $\mathrm{C}$ & 3.20983179801121 & 6.17982348473594 & 12.62848872409745 \\
\hline $\mathrm{H}$ & 4.06970434817313 & 5.91290250639727 & 13.25750192294608 \\
\hline $\mathrm{H}$ & 3.08166850092724 & 5.36686148572922 & 11.90028353152240 \\
\hline $\mathrm{C}$ & 3.48184792387416 & 7.48877741656384 & 11.88111093171584 \\
\hline $\mathrm{H}$ & 4.36795950053904 & 7.38648970655437 & 11.24107276029228 \\
\hline $\mathrm{H}$ & 3.70285141069686 & 8.28060999535771 & 12.61200138396954 \\
\hline $\mathrm{C}$ & 2.26833266705912 & 7.90756317124779 & 11.04597461454254 \\
\hline $\mathrm{H}$ & 2.46100340817808 & 8.86510654307279 & 10.54385420117626 \\
\hline $\mathrm{H}$ & 2.09850862735561 & 7.16053825455282 & 10.25628669171662 \\
\hline $\mathrm{C}$ & 1.00703331335588 & 8.01862211242731 & 11.91163818201298 \\
\hline $\mathrm{H}$ & 0.14959518141954 & 8.29388189396176 & 11.28577568694701 \\
\hline $\mathrm{H}$ & 1.14663528361148 & 8.82643261423623 & 12.64178657963946 \\
\hline $\mathrm{C}$ & -2.18929913536181 & 7.13639461239217 & 12.61584181703429 \\
\hline $\mathrm{H}$ & -2.00071415805558 & 8.14686544233435 & 12.23042565138398 \\
\hline $\mathrm{C}$ & -2.31231494217881 & 6.18460778182442 & 11.41796052050965 \\
\hline $\mathrm{H}$ & -1.40153029868131 & 6.21391868179632 & 10.81040209551238 \\
\hline $\mathrm{H}$ & -2.40875456158320 & 5.15789088241107 & 11.79441703687693 \\
\hline $\mathrm{C}$ & -3.52707192861717 & 6.53334668526554 & 10.55061193615490 \\
\hline $\mathrm{H}$ & -3.37288517498111 & 7.52297325332861 & 10.09407271093293 \\
\hline $\mathrm{H}$ & -3.61091887721875 & 5.81400408739740 & 9.72576766865931 \\
\hline $\mathrm{C}$ & -4.81854583316107 & 6.55477637960138 & 11.37187187170433 \\
\hline $\mathrm{H}$ & -5.02385208293559 & 5.53999149080683 & 11.74462971261822 \\
\hline $\mathrm{H}$ & -5.66801423010623 & 6.84047414125275 & 10.73791006524226 \\
\hline $\mathrm{C}$ & -4.69848987289551 & 7.50630064661513 & 12.56520972118180 \\
\hline $\mathrm{H}$ & -5.61324698086816 & 7.47445333552992 & 13.17182102055361 \\
\hline $\mathrm{H}$ & -4.59434865815248 & 8.53890725617899 & 12.19927542047951 \\
\hline $\mathrm{C}$ & -3.48864227242354 & 7.15091592727606 & 13.43263370778234 \\
\hline $\mathrm{H}$ & -3.62725986442604 & 6.15129154414614 & 13.86316992350973 \\
\hline $\mathrm{H}$ & -3.39672087577689 & 7.84471664006240 & 14.27660796909926 \\
\hline $\mathrm{C}$ & -0.58601873401283 & 8.06581361291875 & 14.85325394295044 \\
\hline $\mathrm{C}$ & -0.77070020070862 & 9.37795501008319 & 14.39315498052411 \\
\hline $\mathrm{H}$ & -1.12714082790008 & 9.54760245250321 & 13.38231460309430 \\
\hline $\mathrm{C}$ & -0.49440871455389 & 10.48433425214366 & 15.19609873197555 \\
\hline $\mathrm{H}$ & -0.65152349016445 & 11.48831717682197 & 14.80790725384687 \\
\hline
\end{tabular}




$\begin{array}{llcc}\mathrm{C} & -0.00123746429935 & 10.29300633252486 & 16.48599345889801 \\ \mathrm{H} & 0.23753174851986 & 11.14513999667419 & 17.11814014752931 \\ \mathrm{C} & 0.16853201456842 & 8.99673511491210 & 16.96842254667603 \\ \mathrm{H} & 0.52633027951614 & 8.83830989500864 & 17.98323898019171 \\ \mathrm{C} & -0.13874900640742 & 7.87560693105424 & 16.18270153097612 \\ \mathrm{C} & 0.03699327152098 & 6.52141279888669 & 16.77560726856249 \\ \mathrm{C} & 1.31888101513815 & 6.08597496686603 & 17.11682571412963 \\ \mathrm{H} & 2.15527599204183 & 6.76075795431592 & 16.95055688322642 \\ \mathrm{C} & 1.54994722791564 & 4.79576802647517 & 17.60217782991743 \\ \mathrm{H} & 2.55859712447525 & 4.48025890699080 & 17.85603339321919 \\ \mathrm{C} & 0.47584439578277 & 3.92423872193190 & 17.74558014098394 \\ \mathrm{H} & 0.63397421784214 & 2.91422673717649 & 18.11582721164089 \\ \mathrm{C} & -0.81913963477125 & 4.34148522819354 & 17.42987369363658 \\ \mathrm{H} & -1.64837291790606 & 3.66426119251509 & 17.60170864561496 \\ \mathrm{C} & -1.07491216481819 & 5.65512897350648 & 16.98463336933914 \\ \mathrm{C} & -2.84026861012090 & 7.19056741686573 & 17.69153441234514 \\ \mathrm{H} & -2.06901344403885 & 7.95342866945563 & 17.81005659718928 \\ \mathrm{H} & -3.10086387297409 & 6.79742182532211 & 18.69109646499335 \\ \mathrm{H} & -3.73135172957145 & 7.66568983234027 & 17.26507689507573 \\ \mathrm{C} & -3.41718558692881 & 5.14479466921701 & 16.52555630868420 \\ \mathrm{H} & -3.70858269726141 & 4.55542792969371 & 17.41477056119820 \\ \mathrm{H} & -3.04955952815886 & 4.47009419385929 & 15.74045452617317 \\ \mathrm{H} & -4.30986812148767 & 5.65981565354911 & 16.15607774056316 \\ \mathrm{~F} & -1.83839448708260 & 1.72243397624600 & 15.14306426056294 \\ \mathrm{C} & -0.78548545075580 & 2.26271757533666 & 14.41854804057608 \\ \mathrm{C} & -1.03903668325847 & 2.75383333790971 & 13.10504383116000 \\ \mathrm{C} & 0.52417662670631 & 1.94816899413074 & 14.82481267227138 \\ \mathrm{C} & 0.05480394784296 & 2.86202360539802 & 12.21414092969806 \\ \mathrm{H} & -2.05438358521573 & 2.77496094137138 & 12.72158313442206 \\ \mathrm{C} & 1.58501828106317 & 2.10863442146847 & 13.94318076070087 \\ \mathrm{H} & 0.68881905453628 & 1.55361372417522 & 15.82214271809289 \\ \mathrm{C} & 1.34378784772735 & 2.54300926339269 & 12.62096962749448 \\ \mathrm{H} & -0.11974849676707 & 3.15525239935226 & 11.18253810519718 \\ \mathrm{H} & 2.58679881531980 & 1.87350922176566 & 14.28362795623072 \\ \mathrm{O} & 2.33269497407994 & 2.69569858027577 & 11.67619023476570 \\ \mathrm{H} & 3.67279825974870 & 2.38742509098005 & 12.05890039523652 \\ \mathrm{H} & 3.77559356165133 & 1.33073678500300 & 12.34246723682898 \\ & 4.00626942274507 & 3.02086017217072 & 12.89253873611574\end{array}$

\section{TS $_{6-7} \mathrm{Cl}$ (Pd(0)-DavePhos complex with Pd-aryl binding with 4-methoxychlorobenzene oxidative addition TS)}

Final Gibbs free enthalpy $\left(\Delta \mathrm{G}=\Delta \mathrm{H}-\mathrm{T}^{*} \Delta \mathrm{S}\right)=-2340.73144864 E_{\mathrm{h}}$

$\begin{array}{cccc}\text { Pd }-1.08818065493466 & 4.94645809216243 & 14.52825260534282 \\ \text { P }-0.50929970215323 & 3.22007833794358 & 15.84073624536928\end{array}$




\begin{tabular}{|c|c|c|c|}
\hline & & & \\
\hline & -1.78178060951614 & & 16.24600353625794 \\
\hline & 0.78842601703315 & 2.26318125092311 & \\
\hline & & & 7373400603 \\
\hline & .35322542887172 & 5.02127160195184 & 42044 \\
\hline$C$ & & & \\
\hline & -1.28506223297787 & & 500898 \\
\hline C & -2.87075976219616 & & 414320 \\
\hline & -2.39993511279503 & & 870 \\
\hline & & & \\
\hline & & 2.78 & 13.76 \\
\hline $\mathrm{H}$ & -0.03 & & $17.205^{\prime}$ \\
\hline $\mathrm{H}$ & & & 191 \\
\hline & 0.14 & 5.42 & 19.3460 \\
\hline E & & & \\
\hline & 2.12 & & 17.3 \\
\hline $\mathrm{H}$ & & & \\
\hline 1 & -2.4 & & 404 \\
\hline $\mathrm{H}$ & & & \\
\hline $\mathrm{C}$ & & & 370 \\
\hline & -3.5 & & \\
\hline $\mathrm{H}$ & & & \\
\hline & -1.6 & & 932 \\
\hline $\mathrm{H}$ & 0.7 & & \\
\hline & & & \\
\hline $\mathrm{C}$ & & & \\
\hline & & & \\
\hline $\mathrm{H}$ & & & \\
\hline $\mathrm{H}$ & & & 315 \\
\hline & & & 19. \\
\hline $\mathrm{H}$ & & & \\
\hline $\mathrm{A}$ & & & \\
\hline $\mathrm{H}$ & & & 592 \\
\hline $\mathrm{H}$ & & & 809 \\
\hline $\mathrm{C}$ & & & \\
\hline $\mathrm{H}$ & -3.9 & & 7699 \\
\hline $\mathrm{H}$ & & & \\
\hline $\mathrm{H}$ & 2.27 & -0.82 & 14.9650 \\
\hline & & & 13.4330 \\
\hline H & 2.64 & & 12.09949 \\
\hline & & & \\
\hline $\mathrm{C}$ & $0.6717770^{\prime}$ & & 11.95462 \\
\hline & & & \\
\hline $\mathrm{H}$ & 2.07184295556430 & 6.18195224748097 & 18.74779075676312 \\
\hline & -5.09454773420679 & 1.74898981465979 & 15.62358350831675 \\
\hline & -5.34025330768255 & 0.17581254952009 & 16.38583922703581 \\
\hline
\end{tabular}




$\begin{array}{llcc}\mathrm{H} & 3.22309411909332 & 0.07240769750386 & 12.83495943229101 \\ \mathrm{H} & 2.15215106413500 & 5.02263892584927 & 14.97611429807446 \\ \mathrm{C} & 1.87789969396237 & 6.52429697804341 & 13.46541860512365 \\ \mathrm{~N} & 0.07690364515540 & 3.43553262353928 & 11.21070844037377 \\ \mathrm{C} & 0.82962880119786 & 5.75087471324751 & 11.42786423997128 \\ \mathrm{H} & 2.35090349706851 & 7.31058973358914 & 14.04798169378023 \\ \mathrm{C} & 1.42706711508434 & 6.76876272945899 & 12.16812344380395 \\ \mathrm{C} & -0.95605220861723 & 2.61834317581237 & 11.83826155979049 \\ \mathrm{C} & -0.09244073725399 & 3.60475177932731 & 9.78114232090773 \\ \mathrm{H} & 0.45722309299697 & 5.97301021200462 & 10.43372527564422 \\ \mathrm{H} & 1.52779170302326 & 7.75878980874016 & 11.72826655162270 \\ \mathrm{H} & -1.08556600972408 & 2.93174538145792 & 12.87709475539210 \\ \mathrm{H} & -0.71369838017610 & 1.54779771204943 & 11.80522732387928 \\ \mathrm{H} & -1.92012518073595 & 2.77563765721341 & 11.32798013133402 \\ \mathrm{H} & 0.82717639694742 & 3.98986024701501 & 9.33023278659436 \\ \mathrm{H} & -0.92551292433915 & 4.28412782863374 & 9.52108965912284 \\ \mathrm{H} & -0.30829012099450 & 2.62559860313302 & 9.33974308022855 \\ \mathrm{C} & -3.22350390597759 & 5.79623872962360 & 14.26956761371557 \\ \mathrm{C} & -3.92418386565081 & 4.86655438435377 & 13.48549416719024 \\ \mathrm{C} & -3.72688335260973 & 6.20807084233885 & 15.50688933668704 \\ \mathrm{C} & -5.06122585344693 & 4.26396401956681 & 14.00512254270682 \\ \mathrm{H} & -3.55974809693868 & 4.59744798149531 & 12.50002611235580 \\ \mathrm{C} & -4.87173119593073 & 5.59963781737942 & 16.02017395771724 \\ \mathrm{H} & -3.20892381418659 & 6.96951365388612 & 16.08052375147178 \\ \mathrm{C} & -5.54177472117550 & 4.61908123079165 & 15.27455981443581 \\ \mathrm{H} & -5.59523080507365 & 3.51239211201194 & 13.42946792488508 \\ \mathrm{H} & -5.22702308080207 & 5.90176335061412 & 16.99857693658170 \\ \mathrm{O} & -6.66915565507402 & 3.96058338775754 & 15.69406895307885 \\ \mathrm{C} & -7.13134535065962 & 4.21680311612881 & 17.02171228344892 \\ \mathrm{H} & -7.44692272140788 & 5.26236659452883 & 17.13935886569879 \\ \mathrm{H} & -7.98938321799939 & 3.55805431253427 & 17.17010585020622 \\ \mathrm{H} & -6.35386897838275 & 3.98299693848861 & 17.76161339515762 \\ \mathrm{Cl} & -2.02098217534765 & 6.92148672219820 & 13.38106785116090\end{array}$

TS $_{6-7}$ F (Pd(0)-DavePhos complex with Pd-aryl binding with 4-methoxyfluorobenzene oxidative addition TS)

Final Gibbs free enthalpy $\left(\Delta \mathrm{G}=\Delta \mathrm{H}-\mathrm{T}^{*} \Delta \mathrm{S}\right)=-1980.29533050 E_{\mathrm{h}}$

$\begin{array}{cccc}\text { Pd } & -1.04405012730384 & 4.91129723750632 & 14.41073111536204 \\ \text { P } & -0.50137678195115 & 3.20569607448854 & 15.76444143808182 \\ \text { C } & 0.13542328580958 & 3.71098315152492 & 17.45314679573585 \\ \text { C } & -1.76362279656008 & 1.90674197444061 & 16.16233750879333 \\ \text { C } & 0.85688692475292 & 2.27010485608949 & 14.95014773410502 \\ \text { H } & -0.23573127765404 & 2.94939300729967 & 18.15460056362197 \\ \text { C } & -0.46733152215248 & 5.07267505648910 & 17.83980766829185 \\ \text { C } & 1.66339667761831 & 3.74778430469416 & 17.56921012645603\end{array}$




\begin{tabular}{|c|c|c|c|}
\hline & -1.27660207677180 & & \\
\hline & & & \\
\hline & -2.29191600860319 & & \\
\hline & 1.23836622521841 & & \\
\hline & 1.46703184904435 & 2.77780809266795 & \\
\hline & -0.14475196072676 & 5.80908099212282 & 9013276805512 \\
\hline & -1.559210 & & \\
\hline & -0.026287 & & \\
\hline & 2.100075 & & \\
\hline & 2.090591 & 2.77 & \\
\hline & $2.06^{\prime}$ & & \\
\hline & -2.54 & 2.9322 & 707 \\
\hline & -3.33 & & \\
\hline & -4.01 & & 649 \\
\hline & -3.38 & & \\
\hline & -2.70 & & \\
\hline & -1.46 & & \\
\hline & 0.78 & & \\
\hline & 2.18 & & \\
\hline & 2.41 & & \\
\hline & 1.206 & & \\
\hline $\mathrm{H}$ & -0.44 & & \\
\hline & -0.44 & & \\
\hline & 1.498 & 5.52 & \\
\hline $\mathrm{H}$ & & & \\
\hline & 1.77 & 3.41 & \\
\hline & -3.61 & & \\
\hline & -4.8 & & \\
\hline$y$ & -4.5 & 38 & \\
\hline & -3.7 & & \\
\hline $\mathrm{H}$ & & & \\
\hline $\mathrm{I}$ & 2.46 & & \\
\hline & $2.76^{\circ}$ & & \\
\hline $\mathrm{H}$ & 2.867 & & \\
\hline & 1.627 & & \\
\hline & 0.71 & 4.35 & \\
\hline & 1.79 & & \\
\hline $\mathrm{H}$ & 1.903843 & 6.30669 & \\
\hline H & -5.02085 & 78608988 & \\
\hline $\mathrm{H}$ & -5.28936 & & 16 . \\
\hline H & 3.505318 & 578057553 & \\
\hline & 2.047312 & & \\
\hline $\mathrm{C}$ & 1.615853 & 6.55626721167449 & 96790 \\
\hline & 0.275928 & & \\
\hline & 0.71141772248799 & 5.66807306179567 & 11.39513948457508 \\
\hline & 1.98230599570565 & 7.38959729826237 & 14.04676563679464 \\
\hline
\end{tabular}




$\begin{array}{llcc}\text { C } & 1.16427404069317 & 6.74925032535516 & 12.15133487294580 \\ \text { C } & -0.77006651030248 & 2.42166968634337 & 11.68935677127295 \\ \text { C } & 0.08910191923254 & 3.49459079754988 & 9.70283053442421 \\ \text { H } & 0.33258476419719 & 5.85398960556643 & 10.39672369180962 \\ \text { H } & 1.14606107020456 & 7.74582819917778 & 11.71580840732492 \\ \text { H } & -0.56187416295118 & 2.18994199738839 & 12.72989313529108 \\ \text { H } & -0.81440724293289 & 1.48354633517489 & 11.12687747440443 \\ \text { H } & -1.75695364277635 & 2.91391616419965 & 11.64099481679456 \\ \text { H } & 0.96819787317500 & 3.98777568215260 & 9.27751499869025 \\ \text { H } & -0.80500566408732 & 4.10306449450558 & 9.47107515971257 \\ \text { H } & -0.03228611865900 & 2.52125309878797 & 9.21683133240687 \\ \text { C } & -3.09799489559340 & 5.47100823691023 & 14.48330493018831 \\ \text { C } & -3.94098413857910 & 4.62200551679186 & 13.74520347599868 \\ \text { C } & -3.55762339588409 & 6.05661612282355 & 15.66903475379120 \\ \text { C } & -5.15543658383168 & 4.22737612852663 & 14.28831029289783 \\ \text { H } & -3.62302356737909 & 4.24712719332707 & 12.77768181668324 \\ \text { C } & -4.78127672086690 & 5.65274660991867 & 16.20783437013944 \\ \text { H } & -2.94359709011144 & 6.78262693726927 & 16.19251381416397 \\ \text { C } & -5.57889601500685 & 4.71984095802996 & 15.53205735671480 \\ \text { H } & -5.79144605734295 & 3.52517623511111 & 13.75443808960162 \\ \text { H } & -5.09287078168175 & 6.07206887298830 & 17.15828952470768 \\ \text { O } & -6.78613962609746 & 4.23785648057169 & 15.99475282351583 \\ \text { C } & -7.18042308062886 & 4.61891128858159 & 17.31101776416853 \\ \text { H } & -7.34979299785900 & 5.70256231064189 & 17.38385777020957 \\ \text { H } & -8.11708267417935 & 4.09245169871083 & 17.50867570332645 \\ \text { H } & -6.42531063549698 & 4.31956526063195 & 18.05237812161309 \\ \text { F } & -2.30029573539389 & 6.51734568433763 & 13.50550896747437\end{array}$

7Cl (Pd(II)-DavePhos complex with Pd-aryl binding with 4-methoxybenzene and chlorine bound)

Final Gibbs free enthalpy $\left(\Delta \mathrm{G}=\Delta \mathrm{H}-\mathrm{T}^{*} \Delta \mathrm{S}\right)=-2340.78150791 E_{\mathrm{h}}$

$\begin{array}{rlcc}\text { Pd } & -1.26519475916393 & 4.83011778472216 & 14.28142792888804 \\ \text { P } & -0.58436760227257 & 3.18777390113288 & 15.66634075776671 \\ \text { C } & -0.18595693585332 & 3.86881103736843 & 17.33783833716616 \\ \text { C } & -1.63759948560984 & 1.69739029951418 & 16.01526721432964 \\ \text { C } & 0.90737285633283 & 2.45268762233782 & 14.89607803058781 \\ \text { H } & -1.19270741052005 & 4.06491547260079 & 17.73460430817726 \\ \text { C } & 0.53122170394232 & 5.22157033141488 & 17.26223100457179 \\ \text { C } & 0.53303131218312 & 2.92122281686689 & 18.30505894869449 \\ \text { H } & -0.96015170625308 & 0.97743289154238 & 16.49528563927263 \\ \text { C } & -2.79428220973179 & 1.96490468579966 & 16.98886542680213 \\ \text { C } & -2.12490503192371 & 1.07209517379566 & 14.70101763078692 \\ \text { C } & 1.49082784633852 & 1.28537921645794 & 15.41727745715579 \\ \text { C } & 1.38959876881561 & 2.98608919886275 & 13.68079925869479 \\ \text { H } & 1.54575316274383 & 5.07124198744852 & 16.87007064754419\end{array}$




\begin{tabular}{|c|c|c|c|}
\hline & 0.00769236543999 & & \\
\hline & & & \\
\hline & 63225643260738 & & \\
\hline & & & \\
\hline & .54333431390083 & & 17.92557098048863 \\
\hline & -2.41006403289446 & .33570987068301 & 57886238 \\
\hline & 3.44761281990595 & & \\
\hline & 61396897387941 & 0.69256 & 1277237 \\
\hline & & & \\
\hline & 261417 & 18535790 & 5013 \\
\hline & -1.26 & & 061 \\
\hline & 3526 & 47 & 188 \\
\hline & & & \\
\hline & 671 & & \\
\hline & & & \\
\hline $\mathrm{H}$ & 146 & & \\
\hline & 5277 & & 162 \\
\hline & & & \\
\hline & 1.17 & & 68 \\
\hline H & & & 965 \\
\hline & -2.98 & & 7497 \\
\hline $\mathrm{H}$ & -4.44 & & 620 \\
\hline & & & \\
\hline $\mathrm{H}$ & & & 897 \\
\hline $\mathrm{H}$ & & & 036 \\
\hline & 2.95 & -0.2 & 783 \\
\hline & & & \\
\hline & 2.78 & & \\
\hline & & & 63 \\
\hline & 0.56 & & \\
\hline $\mathrm{H}$ & & & \\
\hline $\mathrm{H}$ & 2.363 & & \\
\hline $\mathrm{H}$ & -4.81 & & \\
\hline $\mathrm{H}$ & & 116 & 532 \\
\hline & & & \\
\hline & 1.74 & & 136 \\
\hline & & & \\
\hline & 0.1201 & 6475 & 049 \\
\hline & & & \\
\hline & & & \\
\hline & 1.12459656289266 & 6.8379 & 3399 \\
\hline & & & 47168 \\
\hline & -0.100461 & 24144690 & 2596206 \\
\hline & & & \\
\hline & 1.19231105221026 & 7.80526919786952 & 11.40770631622447 \\
\hline & -0.59622745529219 & 2.26362034513420 & 12.64518302810403 \\
\hline
\end{tabular}




$\begin{array}{llcl}\mathrm{H} & -0.86474925126265 & 1.52478268367486 & 11.06303147491440 \\ \mathrm{H} & -1.86716125289704 & 2.90922490791226 & 11.59162396863223 \\ \mathrm{H} & 0.78044879026001 & 3.99914514118697 & 9.10950139855699 \\ \mathrm{H} & -0.97927105097900 & 4.18337497858624 & 9.35862611888319 \\ \mathrm{H} & -0.26773433424923 & 2.56754029060499 & 9.13286083419080 \\ \mathrm{C} & -3.05288659447991 & 4.80539827592281 & 15.16745438203652 \\ \mathrm{C} & -4.13165844685091 & 4.19738299815095 & 14.52002860647678 \\ \mathrm{C} & -3.26739620941867 & 5.43612148805174 & 16.38709113747016 \\ \mathrm{C} & -5.39195846304589 & 4.17592856975583 & 15.10946278300925 \\ \mathrm{H} & -3.99732220515829 & 3.72951596259570 & 13.54959023642645 \\ \mathrm{C} & -4.53008194275072 & 5.42135929548456 & 16.99567386848798 \\ \mathrm{H} & -2.45418427635459 & 5.94534767943958 & 16.89653820082556 \\ \mathrm{C} & -5.59598523521573 & 4.77727432104966 & 16.35991938203585 \\ \mathrm{H} & -6.22950952258840 & 3.69070624473922 & 14.61383262848451 \\ \mathrm{H} & -4.65688287050244 & 5.90895918854374 & 17.95601401524851 \\ \mathrm{O} & -6.86712039216488 & 4.68566432932353 & 16.87140732009551 \\ \mathrm{C} & -7.10751646675749 & 5.27130857063413 & 18.15272218471124 \\ \mathrm{H} & -6.92562173014133 & 6.35515077757203 & 18.13625007470299 \\ \mathrm{H} & -8.15939521353307 & 5.07814114226487 & 18.37565903468461 \\ \mathrm{H} & -6.47796522283793 & 4.80788704632622 & 18.92491366779540 \\ \mathrm{Cl} & -2.10357079020029 & 6.60205634464163 & 12.91297807654655\end{array}$

7F (Pd(II)-DavePhos complex with Pd-aryl binding with 4-methoxybenzene and fluorine bound)

Final Gibbs free enthalpy $\left(\Delta \mathrm{G}=\Delta \mathrm{H}-\mathrm{T}^{*} \Delta \mathrm{S}\right)=-1980.34513510 E_{\mathrm{h}}$

$\begin{array}{cccc}\mathrm{Pd} & -1.23010741294759 & 4.81579489558699 & 14.31765774971879 \\ \mathrm{P} & -0.56059412868651 & 3.18478508444439 & 15.67856468677235 \\ \mathrm{C} & -0.14648265833288 & 3.84849334726467 & 17.35419981512687 \\ \mathrm{C} & -1.62015262102046 & 1.69951908576577 & 16.02846085073100 \\ \mathrm{C} & 0.92586337001749 & 2.44746916139214 & 14.89503832399508 \\ \mathrm{H} & -1.14908549231803 & 4.05218840673917 & 17.75802235712490 \\ \mathrm{C} & 0.58261506428885 & 5.19535606057752 & 17.28355027970239 \\ \mathrm{C} & 0.56824798947477 & 2.88807557855249 & 18.31197512316776 \\ \mathrm{H} & -0.95256839811618 & 0.98160944072658 & 16.52520166602875 \\ \mathrm{C} & -2.78941712743471 & 1.98508718579020 & 16.98213307324899 \\ \mathrm{C} & -2.09718169644030 & 1.06197354771045 & 14.71649470941606 \\ \mathrm{C} & 1.51085153082921 & 1.27695671444160 & 15.40568661141208 \\ \mathrm{C} & 1.40441177294052 & 2.98846033934991 & 13.68071842437126 \\ \mathrm{H} & 1.59176470937473 & 5.03959682502180 & 16.87973073274581 \\ \mathrm{H} & 0.05638610274587 & 5.85964345603215 & 16.58919898769476 \\ \mathrm{C} & 0.69103057311621 & 5.82245177480490 & 18.67565445316055 \\ \mathrm{C} & 0.68399511275872 & 3.51274550949299 & 19.70902442689589 \\ \mathrm{H} & 0.04330327150082 & 1.92857775917089 & 18.37504792299970 \\ \mathrm{H} & 1.57403397867619 & 2.68016280825649 & 17.92499949202916 \\ \mathrm{H} & -2.41832858471605 & 2.37217235800426 & 17.93488738519543\end{array}$




\begin{tabular}{|c|c|c|c|}
\hline & -3.43365414962857 & & \\
\hline & & & \\
\hline & .95593047813044 & .18007034932731 & \\
\hline & -2.67722390203958 & & \\
\hline & -1.23199353688501 & 0.78903888707707 & \\
\hline & 1.14679504826129 & & \\
\hline & 2556936301 & & \\
\hline & 31 & & \\
\hline & & & \\
\hline & 1.233 & & \\
\hline & 56560 & & \\
\hline & 4715 & 4.87 & \\
\hline & 1.226 & & \\
\hline & -0.325484 & & 20. \\
\hline & -2.998189 & & \\
\hline I & -4.45 & & \\
\hline & -4.123232 & & \\
\hline & -3.32 & & \\
\hline & -2.32 & -0.9 & \\
\hline $\mathrm{H}$ & 2.972 & $-0.2^{-}$ & \\
\hline & 2.991 & & \\
\hline I & 2.79 & & \\
\hline & 1.34 & & \\
\hline & 0.49 & & \\
\hline $\mathrm{H}$ & 1.40 & & \\
\hline & 2.426 & 4.7 & \\
\hline I & -4.79 & & \\
\hline & -4.70 & & \\
\hline & 3.788 & & \\
\hline & 1.768 & & \\
\hline $\mathrm{C}$ & & & \\
\hline & 0.10 & & \\
\hline & 0.512 & & \\
\hline & 1.65 & & \\
\hline & 0.926 & & \\
\hline $\mathrm{C}$ & -0.83 & & 311 \\
\hline & -0.15 & & \\
\hline I & $0.16^{\circ}$ & 5.91 & 4341 \\
\hline $\mathrm{H}$ & 0.912 & & \\
\hline $\mathrm{H}$ & -0.54 & & 12. \\
\hline 1 & -0.82 & & 64911 \\
\hline H & -1.85 & & \\
\hline . & 0.6908546 & 4.0788 & 9.13106363461790 \\
\hline & -1.07380 & & \\
\hline & -0.26188333952429 & 2.58128024088896 & 9.13359104940748 \\
\hline & -3.02694265153710 & 4.81422274669853 & 15.16546980437006 \\
\hline
\end{tabular}




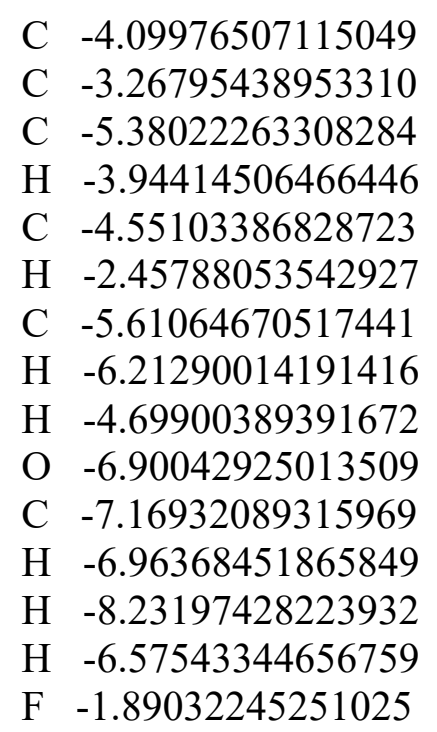

4.24119370699050

5.43685414871809

4.24771036549354

3.77323704710593

5.45244732391254

5.91276656803203

4.84183997670617

3.78758636310036

5.93451846852647

4.77645785922196

5.35115846349070

6.43088344949281

5.17842641314745

4.86438624149343

6.33691776331086
14.47653454158646

16.38509951755880

15.02178717481627

13.50854316590191

16.94934227592446

16.93096495382168

16.27111038131547

14.49478379611270

17.90963498028063

16.73908881107963

18.01955454276746

18.02361724892376

18.20593924448853

18.80530951014905

13.11207080512651

\section{8 (Pd(II)-DavePhos complex with Pd-aryl binding with 4-methoxybenzene and phenyl bound)}

Final Gibbs free enthalpy $\left(\Delta \mathrm{G}=\Delta \mathrm{H}-\mathrm{T}^{*} \Delta \mathrm{S}\right)=-2111.96852077 E_{\mathrm{h}}$

$\begin{array}{clcc}\text { Pd } & -1.32992962306451 & 4.92801467660349 & 15.02473281002907 \\ \text { P } & -0.69075382293573 & 2.92604993136538 & 16.05116680613953 \\ \text { C } & -1.74750177039092 & 2.45793267790962 & 17.49283637867311 \\ \text { C } & -0.68846864405492 & 1.46272997318314 & 14.90743912714243 \\ \text { C } & 1.06615670552095 & 2.97561263375861 & 16.56408224541058 \\ \text { H } & -2.72738513185285 & 2.34254735089288 & 17.00326689530048 \\ \text { C } & -1.87030140219512 & 3.64216510586268 & 18.45887963525859 \\ \text { C } & -1.43392005840849 & 1.16648097242334 & 18.25376380320460 \\ \text { H } & -0.07021717947963 & 0.67253919886304 & 15.35601613784297 \\ \text { C } & -2.09801347585113 & 0.90239114175220 & 14.66816497228405 \\ \text { C } & -0.02456210415084 & 1.91103214675850 & 13.59308310833850 \\ \text { C } & 1.71513447485385 & 1.85790469674548 & 17.11493951141322 \\ \text { C } & 1.82195595999834 & 4.11976591066151 & 16.24318962860198 \\ \text { H } & -0.90062652649161 & 3.80998992591386 & 18.94569188926816 \\ \text { H } & -2.09265985376696 & 4.55093581116179 & 17.89160945980847 \\ \text { C } & -2.94336448154059 & 3.37474345567205 & 19.51535515571742 \\ \text { C } & -2.51862303677718 & 0.88930690229386 & 19.30277130676303 \\ \text { H } & -1.34369044191283 & 0.31515380029000 & 17.56902547978119 \\ \text { H } & -0.47044509365699 & 1.27661812598782 & 18.76698694162077 \\ \text { H } & -2.51164607432970 & 0.50914838631617 & 15.60186711580456 \\ \text { H } & -2.75987865476635 & 1.70871860034795 & 14.33787570690946 \\ \text { C } & -2.08575348987352 & -0.20278156580792 & 13.60659520818824 \\ \text { C } & -0.03597172675769 & 0.80417618558920 & 12.53709276196505 \\ \text { H } & -0.56471716976156 & 2.78999444074747 & 13.21711412872300 \\ \text { H } & 1.00212199650460 & 2.24106893450105 & 13.79235855397625 \\ \text { H } & 1.14791566055345 & 0.95972609837316 & 17.33503009472106\end{array}$




\begin{tabular}{|c|c|c|c|}
\hline & 08649709961259 & & \\
\hline & & & \\
\hline & 26463500398101 & & \\
\hline & 2.99448171538914 & .21655517558979 & 2538532 \\
\hline & .92335336814149 & & 419922 \\
\hline & .67543335761649 & 2.06798751837075 & 04832 \\
\hline & 2.27403453675038 & & \\
\hline & 3.47492836399935 & 01 & 398 \\
\hline & & & \\
\hline & -3.11 & 69012 & 9740 \\
\hline & & & 1666 \\
\hline & .39 & 76972 & 11. \\
\hline & & & \\
\hline & .565 & & 17.78 \\
\hline & & & \\
\hline $\mathrm{I}$ & 5 & & \\
\hline & 1.63 & & 05 \\
\hline 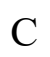 & 0.63 & & \\
\hline & -3.4 & & 587 \\
\hline $\mathrm{H}$ & -1.7 & & 7048 \\
\hline & $-2.0^{\prime}$ & & 7034 \\
\hline & & & \\
\hline & 4.91 & & \\
\hline & 2.12 & & \\
\hline & 1.44 & & \\
\hline & 0.24 & & 17. \\
\hline & & & \\
\hline & 1.73 & & \\
\hline & 0.86 & & 878 \\
\hline & 1.28 & & \\
\hline C & & & \\
\hline & 0.00 & & \\
\hline ה & 0.70 & & 669 \\
\hline 1 & & & 780 \\
\hline & & & \\
\hline $\mathrm{H}$ & 1.80 & & 19.0 \\
\hline & -1.61 & & 5870 \\
\hline . & -0.46 & & 3045 \\
\hline & -1.21 & & 4022 \\
\hline & -3.17 & & 14.5 \\
\hline & -3.48 & 893870 & 3233 \\
\hline$C$ & -4.16 & & 06927 \\
\hline $\mathrm{C}$ & -4.74408270924370 & 8142548 & 12.99712570352003 \\
\hline & & & \\
\hline & -5.42656462322912 & 3.73619305913312 & 15.26819757970523 \\
\hline & -3.96373733661669 & 4.65503073173935 & 16.54367365248772 \\
\hline
\end{tabular}




$\begin{array}{cccc}\mathrm{C} & -5.72102370996335 & 3.24893737995521 & 13.99311316218678 \\ \mathrm{H} & -4.95908325878747 & 2.94796128358112 & 11.99617202484900 \\ \mathrm{H} & -6.18030394973576 & 3.69734108731823 & 16.05336629626969 \\ \mathrm{H} & -6.69959485330234 & 2.82549495598036 & 13.77768113494413 \\ \mathrm{C} & -1.98397587093467 & 6.60081725114004 & 14.07017078221394 \\ \mathrm{C} & -1.63233632707476 & 6.82383273612417 & 12.73892996474345 \\ \mathrm{C} & -2.67759342245768 & 7.62402697819079 & 14.72775749751320 \\ \mathrm{C} & -1.90839485659300 & 8.03210567234151 & 12.08441038495290 \\ \mathrm{H} & -1.12185060158723 & 6.04460048671457 & 12.17516423465270 \\ \mathrm{C} & -2.95987745249897 & 8.83865649536152 & 14.10636996181188 \\ \mathrm{H} & -3.01460449710371 & 7.47944766070307 & 15.75252701955349 \\ \mathrm{C} & -2.55989081675802 & 9.05479982175204 & 12.78018510434211 \\ \mathrm{H} & -1.60377809543025 & 8.16209261972658 & 11.05065559028204 \\ \mathrm{H} & -3.48496476291797 & 9.63368933194540 & 14.63373881482874 \\ \mathrm{O} & -2.86652344505425 & 10.28937371260156 & 12.25007282494459 \\ \mathrm{C} & -2.49023952531502 & 10.53690611881614 & 10.89520645735781 \\ \mathrm{H} & -1.40158626107228 & 10.45773575504295 & 10.76196859069579 \\ \mathrm{H} & -2.98953517499825 & 9.84036338307897 & 10.20750493326084 \\ \mathrm{H} & -2.81216633713528 & 11.55786167852815 & 10.67714412998913\end{array}$

TS8-5 (Pd(II)-DavePhos complex with Pd-aryl binding with 4-methoxybenzene and phenyl reductive elimination $\mathrm{TS}$ )

Final Gibbs free enthalpy $\left(\Delta \mathrm{G}=\Delta \mathrm{H}-\mathrm{T}^{*} \Delta \mathrm{S}\right)=-2111.95766494 E_{\mathrm{h}}$

$\begin{array}{llcc}\text { Pd } & -1.44534510669325 & 4.69420764797788 & 14.93134582756315 \\ \text { P } & -0.73099451644833 & 2.70815570530285 & 15.85993050681006 \\ \text { C } & -1.87790263213636 & 2.17256654935676 & 17.22915967568811 \\ \text { C } & -0.55876108904874 & 1.18693064757669 & 14.81218366850529 \\ \text { C } & 0.92913184834166 & 2.99240215998157 & 16.59343588623620 \\ \text { H } & -2.83716706389695 & 2.15156086558624 & 16.68874466293359 \\ \text { C } & -2.00405006552997 & 3.26824971564631 & 18.29366994373173 \\ \text { C } & -1.67381571419555 & 0.80272353001072 & 17.88603510351953 \\ \text { H } & -0.17976264301211 & 0.36854758849480 & 15.44170461539422 \\ \text { C } & -1.93474428295808 & 0.79358527942564 & 14.24929817803443 \\ \text { C } & 0.43942537027781 & 1.41180550842878 & 13.67121518168557 \\ \text { C } & 1.56044411713370 & 2.03771446996486 & 17.40789014055289 \\ \text { C } & 1.55705690534439 & 4.24619150873228 & 16.38736656767944 \\ \text { H } & -1.05090704335658 & 3.36400382120453 & 18.83246165025589 \\ \text { H } & -2.19571871015540 & 4.22608969846491 & 17.80298449121851 \\ \text { C } & -3.13130844180306 & 2.96004063283723 & 19.28231579341926 \\ \text { C } & -2.82097758666909 & 0.49207840146450 & 18.85710376649124 \\ \text { H } & -1.60521287699921 & 0.00900208737704 & 17.13371590898560 \\ \text { H } & -0.73075837004403 & 0.80340210305855 & 18.44554774745139 \\ \text { H } & -2.64345582199131 & 0.59235613078292 & 15.05935053055864 \\ \text { H } & -2.33594598583649 & 1.64814261747665 & 13.68768233543632 \\ \text { C } & -1.83835491441609 & -0.43026675660035 & 13.33349867464241\end{array}$




\begin{tabular}{|c|c|c|c|}
\hline & 0.54491205172070 & & \\
\hline & & & \\
\hline & 42418027463245 & & \\
\hline & & & \\
\hline & & & \\
\hline & 4839 & & \\
\hline & & & \\
\hline & 3044883 & 10774 & 028 \\
\hline & & & \\
\hline & 0439 & 619 & 19669 \\
\hline & -2.65 & & \\
\hline & 4650 & 5249 & 116 \\
\hline & & & \\
\hline & 5138 & 427 & 4029 \\
\hline & & & \\
\hline $\mathrm{I}$ & 6611 & & \\
\hline & 6587 & & 46 \\
\hline & 37 & & \\
\hline & & & \\
\hline & & & \\
\hline & & & 536 \\
\hline & & & \\
\hline $\mathrm{H}$ & & & 43408 \\
\hline & -2.0 & & \\
\hline $\mathrm{H}$ & & & 6850 \\
\hline & -0.7 & 680 & 2986 \\
\hline & & & \\
\hline & & & 866 \\
\hline & & & 9012 \\
\hline & 29 & & \\
\hline & & & \\
\hline & & & \\
\hline & & & \\
\hline & -0.1 & & 96246 \\
\hline & 0.76 & & \\
\hline & 0.72 & & 5740 \\
\hline & & & \\
\hline $\mathrm{H}$ & 0.46 & & 21103 \\
\hline $\mathrm{I}$ & -0.74 & & 272500 \\
\hline $\mathrm{H}$ & & & 353675 \\
\hline $\mathrm{H}$ & 809031673 & 5800 & 8879604 \\
\hline & & & 16.9628247 \\
\hline H & 0995025350 & 3968 & 18.50084699986470 \\
\hline & & & \\
\hline & -3.94679414649777 & 4.07945357485005 & 13.49075268885063 \\
\hline & -4.31717550603949 & 4.70846846948714 & 15.78810443952606 \\
\hline
\end{tabular}




$\begin{array}{ll}C & -5.16933856884365 \\ H & -3.33898077430580 \\ C & -5.54031824649022 \\ H & -4.00980405460304 \\ C & -5.97413980612733 \\ H & -5.49083219574127 \\ H & -6.15279175523574 \\ H & -6.92847200411740 \\ C & -2.47676684045301 \\ C & -2.31270694901495 \\ C & -2.59184705863016 \\ C & -2.23959463514587 \\ H & -2.23937657805507 \\ C & -2.51728196958110 \\ H & -2.75417740124529 \\ C & -2.32623600612446 \\ H & -2.09305809771116 \\ H & -2.59103675486902 \\ O & -2.24229226130544 \\ C & -2.01400315848523 \\ H & -1.06655920326293 \\ H & -2.83645239371881 \\ H & -1.96289636589868\end{array}$

3.41077168039417 4.09340355622978 4.03967901395110 5.22616599543812 3.37467904588130 2.90087840483180 4.02563073059676 2.85352119721318 6.41468621951359 6.83453388140415 7.40162865727595 8.18861326122114 6.09652231865314 8.75212475978291 7.11319790757147 9.15441938378501 8.47057285983552 9.51365341775127 10.50829020137359 10.96009429180583 10.56793241117578 10.66529512842456 12.04991515511817
13.47702236604772

12.59017045324626

15.77301425428634

16.69197145390517

14.62125711732819

12.57014966746083

16.67349658336758

14.61004171448706

14.34521562462209

13.02158696543830

15.33871539954506

12.68433892285652

12.22702602027933

15.02532937053876

16.37387470339355

13.69400094758879

11.64716015031955

15.79830527258291

13.48401943333115

12.14740785387091

11.75316515055308

11.48022376538250

12.20674770813860

\section{9 (Pd(0)-DavePhos complex with Pd-aryl binding with PhMgBr and biphenyl bound)}

Final Gibbs free enthalpy $\left(\Delta \mathrm{G}=\Delta \mathrm{H}-\mathrm{T}^{*} \Delta \mathrm{S}\right)=-5119.17964829 E_{\mathrm{h}}$

$\begin{array}{llcc}\text { Pd } & -1.12607556460943 & 4.75978316965362 & 15.69586682698662 \\ \text { P } & -0.40307279493654 & 2.60128815432335 & 15.83604559885400 \\ \text { C } & -1.67557037768209 & 1.52290284626662 & 16.65955079637937 \\ \text { C } & 0.22629746436593 & 1.57996437050150 & 14.41561668565898 \\ \text { C } & 1.08033549967228 & 2.66070299065698 & 16.93342804859858 \\ \text { H } & -2.45866302212221 & 1.51268411852082 & 15.88699504841571 \\ \text { C } & -2.28572323224706 & 2.22782113883811 & 17.87617227786349 \\ \text { C } & -1.33011046825140 & 0.07019982317889 & 16.99508347712182 \\ \text { H } & 0.67304368099983 & 0.68284896536117 & 14.86499583945914 \\ \text { C } & -0.88564676544944 & 1.12576198106371 & 13.46057218529579 \\ \text { C } & 1.32387595861000 & 2.34509897225446 & 13.66470365915935 \\ \text { C } & 1.67561813817840 & 1.45652174959454 & 17.34871920044162 \\ \text { C } & 1.61273564339257 & 3.88274361371920 & 17.40565278753280 \\ \text { H } & -1.54212320283638 & 2.26307266464134 & 18.68431286987381 \\ \text { H } & -2.52471483708439 & 3.26048351094366 & 17.60189380657105 \\ \text { C } & -3.53563791164249 & 1.49082287259178 & 18.36197912897822 \\ \text { C } & -2.58900010082325 & -0.67558200259982 & 17.46291844378124 \\ \text { H } & -0.89110819595636 & -0.44411068175322 & 16.13310177444026 \\ \text { H } & -0.58868632516385 & 0.04934720876837 & 17.80314291593194 \\ \text { H } & -1.64622167377354 & 0.54216671726939 & 13.98901514676032\end{array}$




\begin{tabular}{|c|c|c|c|}
\hline & -1.38260109033849 & & \\
\hline & & & \\
\hline & & & \\
\hline & .89582917906814 & & \\
\hline & 2.13025147437784 & & \\
\hline & 1.30908242925483 & & \\
\hline & 2.72961234909128 & & \\
\hline & 2.671615 & & \\
\hline & 1.2260748 & & \\
\hline & -3.9387493 & 1.990091 & 47596 \\
\hline & -4.30425 & & 40346 \\
\hline & -3.23389 & 4723 & 35314 \\
\hline & -2.3 & & 5064 \\
\hline & -3.31098 & -0.7155 & 062285 \\
\hline & 0.103604 & -0.62823249 & 12.67 \\
\hline $\mathrm{H}$ & -1.13458 & & \\
\hline & 0.767206 & 1.090 & 11.5 \\
\hline & 2.63 & & \\
\hline & 2.39 & & \\
\hline & 3.16 & & \\
\hline & 3.21 & & \\
\hline & 3.07 & & \\
\hline & 1.52 & & \\
\hline & 0.85 & 6.29 & \\
\hline $\mathrm{H}$ & & & 191 \\
\hline & -2.54 & 2261 & 378 \\
\hline I & & & \\
\hline & 1.17 & & \\
\hline $\mathrm{H}$ & 4.04 & & \\
\hline & & & \\
\hline $\mathrm{C}$ & & & \\
\hline & & & \\
\hline & 0.90 & & \\
\hline & & & \\
\hline & & & \\
\hline & -0.3 & 800 & 852 \\
\hline & 0.40 & & \\
\hline . & 0.64 & 8.4367 & 64 \\
\hline $\mathrm{H}$ & 1.2904 & & \\
\hline $\mathrm{H}$ & 0.231 & 4.100 & 20.0 \\
\hline $\mathrm{H}$ & -0.6185( & 0463 & 0513 \\
\hline & -1.2296 & & 5543779 \\
\hline H & -0.51308 & 7.6717291 & 0075032657 \\
\hline & & & \\
\hline H & 0.42690139743623 & 6.61012860724054 & 21.10334500696750 \\
\hline & -2.94510492361326 & 6.88743190254448 & 14.55251970881972 \\
\hline
\end{tabular}




$\begin{array}{llcc}\mathrm{C} & -4.25155321794640 & 6.36664107145596 & 14.67284325403072 \\ \mathrm{C} & -2.11848874161305 & 6.86386162498400 & 15.71756842871461 \\ \mathrm{C} & -4.72164618685425 & 5.83148111202703 & 15.87790892030105 \\ \mathrm{H} & -4.92888497747421 & 6.40990541290310 & 13.82500802442366 \\ \mathrm{C} & -2.60679749441095 & 6.32302362712964 & 16.92572608969715 \\ \mathrm{H} & -1.18012296812255 & 7.40556118123911 & 15.72886890104457 \\ \mathrm{C} & -3.90494687483513 & 5.79620568032126 & 17.00375349716547 \\ \mathrm{H} & -5.73211463244617 & 5.43722075796056 & 15.92387176830524 \\ \mathrm{H} & -2.00879885212901 & 6.41539301787897 & 17.82656826630168 \\ \mathrm{H} & -4.27366977164859 & 5.39023714729420 & 17.94106857829718 \\ \mathrm{C} & -2.46181880648794 & 7.47731611088523 & 13.28930034804197 \\ \mathrm{C} & -3.08516771237635 & 7.18890559102018 & 12.06485856770485 \\ \mathrm{C} & -1.31061495721143 & 8.28704144474242 & 13.24490662629500 \\ \mathrm{C} & -2.58780008267913 & 7.65227880757224 & 10.85307913619439 \\ \mathrm{H} & -3.95549179983400 & 6.54175482150667 & 12.03477470404392 \\ \mathrm{C} & -0.79832681913295 & 8.75744000708088 & 12.04728130233675 \\ \mathrm{H} & -0.79342295545178 & 8.54991318840663 & 14.16043011807366 \\ \mathrm{C} & -1.42084775699638 & 8.42375066217828 & 10.83467876865593 \\ \mathrm{H} & -3.08413128789341 & 7.35973162353768 & 9.93612493790364 \\ \mathrm{H} & 0.10147901191938 & 9.36711726384575 & 12.02904635569051 \\ \mathrm{O} & -0.81502373584067 & 8.88184892654150 & 9.70258281563313 \\ \mathrm{C} & -1.37273177932377 & 8.49000970462393 & 8.44143717516489 \\ \mathrm{H} & -1.39522883245409 & 7.39647477347746 & 8.35012078164455 \\ \mathrm{H} & -2.38553546236683 & 8.89412032675865 & 8.31479022291873 \\ \mathrm{H} & -0.71166069610118 & 8.91294221058996 & 7.68240014364260 \\ \mathrm{C} & -1.83938246320145 & 4.28655085261744 & 12.02237209285711 \\ \mathrm{C} & -2.29553098329871 & 3.83675809455313 & 10.76914391292850 \\ \mathrm{C} & -0.63260944013074 & 5.01621685631024 & 12.01123642543620 \\ \mathrm{C} & -1.60414319606626 & 4.10043877706710 & 9.58041010076262 \\ \mathrm{H} & -3.22317644791279 & 3.26327760416550 & 10.70578037810600 \\ \mathrm{C} & 0.07427423640969 & 5.28693491709980 & 10.83963285096350 \\ \mathrm{H} & -0.24008422301850 & 5.40912022009130 & 12.94970487612657 \\ \mathrm{C} & -0.41207981621906 & 4.82750379330955 & 9.61304609667166 \\ \mathrm{H} & -1.99072984635670 & 3.73702365325014 & 8.62835112904573 \\ \mathrm{H} & 0.99552378870738 & 5.86752867929547 & 10.87628679541672 \\ \mathrm{H} & 0.12953878772781 & 5.03755772116172 & 8.69260829134365 \\ \mathrm{Br} \mathrm{-}-4.69988817983550 & 2.42322751339500 & 14.33802845812474\end{array}$

TS9-10 (Pd(0)-DavePhos complex with Pd-aryl binding with PhMgBr and biphenyl bound, ligand exchange TS)

Final Gibbs free enthalpy $\left(\Delta \mathrm{G}=\Delta \mathrm{H}-\mathrm{T}^{*} \Delta \mathrm{S}\right)=-5119.16571509 E_{\mathrm{h}}$

$\begin{array}{llll}\mathrm{Pd} & -0.92248658174215 & 4.36669465190315 & 14.87793099155859 \\ \mathrm{P} & -0.41527830221468 & 2.33373505084554 & 15.75336302043517 \\ \mathrm{C} & -1.74561236449287 & 1.45189365816724 & 16.71859184565396\end{array}$




\begin{tabular}{|c|c|c|c|}
\hline $\mathrm{C}$ & & & \\
\hline $\mathrm{C}$ & & & \\
\hline 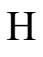 & -2.57862538741643 & 1.49658005960050 & 16.00028315999836 \\
\hline & -2.15852627721939 & & 17.94539503484396 \\
\hline & 1.55969793163497 & -0.01685759390954 & 942531 \\
\hline & & & \\
\hline & & 88877 & 0662 \\
\hline & & & \\
\hline & & 1.3000 & \\
\hline 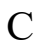 & & & \\
\hline I & & & 5263 \\
\hline & -2.2645 & 3.322 & 3985 \\
\hline & & & \\
\hline & $-2.8^{\prime}$ & -0.5 & 6310 \\
\hline & -1.2 & & \\
\hline & -0.7 & & 541 \\
\hline & -1.7 & & \\
\hline H & $-1.3 \xi$ & & \\
\hline & & & \\
\hline & & & \\
\hline & 0.92 & & \\
\hline 1 & & & \\
\hline & 1.16 & & \\
\hline & & & \\
\hline & & & \\
\hline $\mathrm{C}$ & & & \\
\hline $\mathrm{H}$ & & & 203 \\
\hline $\mathrm{H}$ & $-4.2^{\prime}$ & & \\
\hline C & -3.3 & & 510 \\
\hline I & -2.7 & & 214 \\
\hline $\mathrm{H}$ & -3.6 & & 376 \\
\hline & -0.12 & & 0616 \\
\hline $\mathrm{H}$ & -1.27 & 3321 & 499 \\
\hline & 0.70 & & \\
\hline $\mathrm{H}$ & & & \\
\hline $\mathrm{H}$ & 2.29 & -0.236 & 988 \\
\hline $\mathrm{H}$ & 2.99 & & \\
\hline & $3.17^{\prime}$ & & 230 \\
\hline & & & \\
\hline $\mathrm{C}$ & & & 9871 \\
\hline & & & \\
\hline $\mathrm{H}$ & -4.311410516 & -0.128 & 13170 \\
\hline & -2.627155891 & & 63659 \\
\hline $\mathrm{H}$ & 0.33467386246264 & & \\
\hline & 1.09208243995704 & -0.76234080147784 & 11.15738336714309 \\
\hline & 4.00085618330675 & 2.63224115396347 & 19.43029815981649 \\
\hline
\end{tabular}




\begin{tabular}{|c|c|c|c|}
\hline & & & \\
\hline & & & \\
\hline & & & \\
\hline & & & \\
\hline & 82060170005916 & & \\
\hline & & & \\
\hline & -0.13955678507483 & & 702 \\
\hline & .74376897245289 & 7.277 & 19.5 \\
\hline & & & \\
\hline & 1.28638916905629 & & 14.89 \\
\hline & & & \\
\hline & 12711 & 683 & 475 \\
\hline & & & \\
\hline & 71078 & 184 & 19.404840767 \\
\hline & & & \\
\hline & 0.812 & & \\
\hline & -3.131 & & 763 \\
\hline & -4.3 & & \\
\hline & -2.15 & & 164 \\
\hline & -4.7 & & 595 \\
\hline & 08 & & 253 \\
\hline & & & \\
\hline & -1.22 & & \\
\hline & -3.80 & & \\
\hline & & & \\
\hline & -1.8 & & 854 \\
\hline & -4.0 & & 078 \\
\hline & -2.7 & & 26 \\
\hline & -3.3 & & 938 \\
\hline & -1.8 & & \\
\hline & & & \\
\hline & & & \\
\hline & -1.48 & & 12. \\
\hline & & & 885 \\
\hline & -1.9 & & \\
\hline & -3.3 & & \\
\hline & -0.77 & & \\
\hline & -1.54 & 005 & 26139837 \\
\hline & -2.04 & & 2773 \\
\hline & & & \\
\hline & -3.13 & & 1054 \\
\hline & & & \\
\hline & -1.45187 & 4.54470807366897 & 12.29916592176078 \\
\hline & & & 11.37875208764909 \\
\hline & -0.63976740408842 & 5.69243067357626 & 12.16938517814021 \\
\hline & -0.19070144161226 & 3.60299621912900 & 10.39078694889312 \\
\hline
\end{tabular}




$\begin{array}{llcc}\mathrm{H} & -1.75251950768788 & 2.57815629775955 & 11.40968631885232 \\ \mathrm{C} & 0.36498351746051 & 5.79968179770705 & 11.20199956124367 \\ \mathrm{H} & -0.80631078568960 & 6.54999396883031 & 12.80973370902455 \\ \mathrm{C} & 0.59759698747340 & 4.75447632130628 & 10.30827964327355 \\ \mathrm{H} & -0.03336516543112 & 2.78391435055227 & 9.69047662300982 \\ \mathrm{H} & 0.96600507389515 & 6.70655434814768 & 11.14544677089655 \\ \mathrm{H} & 1.37017007110763 & 4.83987794024383 & 9.54625680334804 \\ \mathrm{Mg} & -3.05262850563251 & 3.96301334408279 & 13.62716081347126 \\ \mathrm{Br} & -4.67312486722061 & 2.12123156525264 & 13.69734608304708\end{array}$

10 (Pd(0)-DavePhos complex with Pd-aryl binding with PhMgBr bound)

Final Gibbs free enthalpy $\left(\Delta \mathrm{G}=\Delta \mathrm{H}-\mathrm{T}^{*} \Delta \mathrm{S}\right)=-4541.69324108 E_{\mathrm{h}}$

$\begin{array}{lcccc}\text { Pd } & 1.91194705793065 & 14.88665734428283 & 29.07089664363240 \\ \mathrm{P} & 3.38293300823859 & 16.31580576591105 & 28.07637130971200 \\ \mathrm{~N} & 0.06189001508676 & 17.84304225758749 & 28.28947670899172 \\ \mathrm{C} & 4.06259246628706 & 17.43705759527225 & 29.40293422620016 \\ \mathrm{H} & 4.50104570675907 & 16.69454916911494 & 30.08840244176909 \\ \mathrm{C} & 2.90700130477781 & 18.10897850887145 & 30.15633674019084 \\ \mathrm{H} & 2.41306056800491 & 18.81970265067588 & 29.48213112422230 \\ \mathrm{H} & 2.16155046164504 & 17.34828002385709 & 30.41718736844781 \\ \mathrm{C} & 3.39774204591979 & 18.84156122619229 & 31.40679375792153 \\ \mathrm{H} & 2.55530011941023 & 19.35056975768359 & 31.89317019304305 \\ \mathrm{H} & 3.78131227885842 & 18.10014060474459 & 32.12361909487328 \\ \mathrm{C} & 4.50761328913108 & 19.84304411062788 & 31.07308834277063 \\ \mathrm{H} & 4.87994401621864 & 20.31967904073140 & 31.98853430809565 \\ \mathrm{H} & 4.09205823432545 & 20.64308109417325 & 30.44126972165503 \\ \mathrm{C} & 5.65669388311048 & 19.16298023583473 & 30.32213865561750 \\ \mathrm{H} & 6.42453027264151 & 19.89969255341003 & 30.05307544814965 \\ \mathrm{H} & 6.13572937478452 & 18.42521231984978 & 30.98301664761639 \\ \mathrm{C} & 5.15378453748718 & 18.45357914194167 & 29.05764137106461 \\ \mathrm{H} & 5.99201128513366 & 17.96693935458827 & 28.54506199115665 \\ \mathrm{H} & 4.74270557746446 & 19.20830931221436 & 28.37518986393841 \\ \mathrm{C} & 4.87247229609898 & 15.37686502748469 & 27.46460646103064 \\ \mathrm{H} & 5.66106860532264 & 16.08843165515076 & 27.18614038615250 \\ \mathrm{C} & 5.39354917718999 & 14.46629312186623 & 28.58646743322044 \\ \mathrm{H} & 5.73810530953556 & 15.06799895335000 & 29.43553728235998 \\ \mathrm{H} & 4.54661633715576 & 13.86241877608738 & 28.94522634689565 \\ \mathrm{C} & 6.52202049211005 & 13.54904357057145 & 28.10709262334333 \\ \mathrm{H} & 7.39875112627507 & 14.15892010281330 & 27.84052727546378 \\ \mathrm{H} & 6.83001403332129 & 12.88964750363419 & 28.92867601196546 \\ \mathrm{C} & 6.09257436295812 & 12.72446605131356 & 26.89203326684568 \\ \mathrm{H} & 5.26758886034755 & 12.05675041832898 & 27.18086186077277 \\ \mathrm{H} & 6.91999023398504 & 12.08881497194533 & 26.55072276091789 \\ \mathrm{C} & 5.61503985385896 & 13.63626864632170 & 25.76048434302492 \\ \mathrm{H} & 5.26844698844905 & 13.03756237325000 & 24.90833896981040\end{array}$




\begin{tabular}{|c|c|c|c|}
\hline & 6.45920910756898 & & \\
\hline $\mathrm{C}$ & & & \\
\hline & & & \\
\hline & 17780215238849 & & \\
\hline & 98047672785500 & & \\
\hline & & & 013 \\
\hline & & & \\
\hline & 837237 & & \\
\hline & & & \\
\hline & 2369 & & 7002 \\
\hline & & & \\
\hline & & & \\
\hline & & & \\
\hline & 93 & & \\
\hline & & & \\
\hline & & & \\
\hline & 77 & & 967 \\
\hline & & & \\
\hline & 23 & & \\
\hline & & & \\
\hline & -2.3 & & \\
\hline & & & \\
\hline & -1.8 & & \\
\hline & -0.1 & & \\
\hline & & & \\
\hline & 0.5 & & \\
\hline & & & \\
\hline & -0.9 & & \\
\hline & 537 & & \\
\hline & -0.3 & & \\
\hline H & & & \\
\hline & -0.0 & & \\
\hline & 0.5 & & \\
\hline & & & \\
\hline & 0.83 & & \\
\hline & -0.4 & & \\
\hline & 1.54 & & \\
\hline $\mathrm{C}$ & -0.96 & & 31. \\
\hline $\mathrm{H}$ & -1.04 & & \\
\hline $\mathrm{C}$ & 1.037 & & \\
\hline $\mathrm{H}$ & 2.5305744 & 12.0084 & 30.206200082 \\
\hline & -0.218 & & 32.1881 \\
\hline & -1.94021730852258 & 13.08553757260098 & 32.06039102559500 \\
\hline & & & 32.06154640702211 \\
\hline & -0.61918790631247 & 11.20176188014644 & \\
\hline
\end{tabular}


11Cl (Pd(0)-DavePhos complex with Pd-aryl binding with PhMgBr bound and ArCl Clbound to Mg)

Final Gibbs free enthalpy $\left(\Delta \mathrm{G}=\Delta \mathrm{H}-\mathrm{T}^{*} \Delta \mathrm{S}\right)=-5347.91701427 E_{\mathrm{h}}$

$\begin{array}{cccc}\mathrm{Pd} & 1.807724 & 14.945362 & 29.345845 \\ \mathrm{P} & 3.458129 & 15.734527 & 27.964026 \\ \mathrm{~N} & 0.855368 & 17.999588 & 28.782936 \\ \mathrm{C} & 4.831181 & 16.376683 & 29.028131 \\ \mathrm{H} & 5.657223 & 16.729574 & 28.398157 \\ \mathrm{C} & 5.368058 & 15.268851 & 29.942098 \\ \mathrm{H} & 4.531498 & 14.868129 & 30.529022 \\ \mathrm{H} & 5.760907 & 14.432155 & 29.356746 \\ \mathrm{C} & 6.451088 & 15.810839 & 30.877139 \\ \mathrm{H} & 6.811348 & 15.009346 & 31.530442 \\ \mathrm{H} & 7.314130 & 16.139836 & 30.278879 \\ \mathrm{C} & 5.935331 & 16.987329 & 31.708795 \\ \mathrm{H} & 6.734914 & 17.380779 & 32.349899 \\ \mathrm{H} & 5.134785 & 16.632478 & 32.376766 \\ \mathrm{C} & 5.377406 & 18.093753 & 30.809968 \\ \mathrm{H} & 4.961915 & 18.908426 & 31.417611 \\ \mathrm{H} & 6.196836 & 18.523529 & 30.215031 \\ \mathrm{C} & 4.304919 & 17.552296 & 29.863297 \\ \mathrm{H} & 3.931385 & 18.346558 & 29.207466 \\ \mathrm{H} & 3.443820 & 17.193723 & 30.444972 \\ \mathrm{C} & 4.229792 & 14.376262 & 26.943871 \\ \mathrm{H} & 4.358983 & 13.579630 & 27.689148 \\ \mathrm{C} & 3.228106 & 13.882244 & 25.891952 \\ \mathrm{H} & 2.261015 & 13.662460 & 26.362995 \\ \mathrm{H} & 3.035575 & 14.690458 & 25.173920 \\ \mathrm{C} & 3.750420 & 12.651404 & 25.147607 \\ \mathrm{H} & 3.821593 & 11.811032 & 25.851591 \\ \mathrm{H} & 3.032380 & 12.354341 & 24.372980 \\ \mathrm{C} & 5.127706 & 12.908935 & 24.530679 \\ \mathrm{H} & 5.032267 & 13.667193 & 23.739024 \\ \mathrm{H} & 5.506941 & 11.996003 & 24.054190 \\ \mathrm{C} & 6.121229 & 13.409495 & 25.582404 \\ \mathrm{H} & 7.089473 & 13.634327 & 25.116898 \\ \mathrm{H} & 6.300702 & 12.616970 & 26.323093 \\ \mathrm{C} & 5.591501 & 14.655578 & 26.303023 \\ \mathrm{H} & 5.491053 & 15.468785 & 25.573547 \\ \mathrm{C} & 6.315202 & 14.984155 & 27.057428 \\ \mathrm{C} & 3.217339 & 17.069271 & 26.712356 \\ \mathrm{H} & 5.323257 & 17.758656 & 26.190779 \\ \mathrm{H} & 5.075204 & 17.595783 & 26.613774 \\ \mathrm{H} & 19.159620 & 24.739186 \\ \mathrm{H} & & & \\ \mathrm{H} & & \end{array}$




$\begin{array}{cccc}\mathrm{H} & 2.835420 & 19.513191 & 23.686634 \\ \mathrm{C} & 1.833788 & 18.193567 & 25.063271 \\ \mathrm{H} & 0.849312 & 18.371992 & 24.637159 \\ \mathrm{C} & 1.942025 & 17.325620 & 26.160925 \\ \mathrm{C} & 0.698925 & 16.708928 & 26.696951 \\ \mathrm{C} & 0.025388 & 15.764001 & 25.918456 \\ \mathrm{H} & 0.435047 & 15.510630 & 24.943724 \\ \mathrm{C} & -1.117752 & 15.112508 & 26.381525 \\ \mathrm{H} & -1.601875 & 14.354006 & 25.773867 \\ \mathrm{C} & -1.604005 & 15.425106 & 27.648578 \\ \mathrm{H} & -2.495254 & 14.931165 & 28.027376 \\ \mathrm{C} & -0.961348 & 16.380432 & 28.433201 \\ \mathrm{H} & -1.358356 & 16.612228 & 29.414487 \\ \mathrm{C} & 0.194026 & 17.042573 & 27.981893 \\ \mathrm{C} & 0.908353 & 19.369221 & 28.284539 \\ \mathrm{H} & 1.171371 & 19.385105 & 27.226814 \\ \mathrm{H} & 1.676788 & 19.920652 & 28.837942 \\ \mathrm{H} & -0.057657 & 19.892347 & 28.414251 \\ \mathrm{C} & 0.610416 & 17.944985 & 30.214564 \\ \mathrm{H} & 0.704932 & 16.906659 & 30.555424 \\ \mathrm{H} & -0.381841 & 18.333022 & 30.509499 \\ \mathrm{H} & 1.372878 & 18.546524 & 30.719569 \\ \mathrm{Mg} & 0.999195 & 12.752708 & 28.771752 \\ \mathrm{Br} & -0.486219 & 11.417323 & 27.341483 \\ \mathrm{C} & 0.588349 & 13.958723 & 30.780384 \\ \mathrm{C} & -0.818252 & 13.816196 & 30.814265 \\ \mathrm{C} & 1.243767 & 13.843024 & 32.030880 \\ \mathrm{C} & -1.521549 & 13.572570 & 31.998994 \\ \mathrm{H} & -1.391081 & 13.901657 & 29.891828 \\ \mathrm{C} & 0.551339 & 13.634978 & 33.224370 \\ \mathrm{H} & 2.327240 & 13.939966 & 32.072620 \\ \mathrm{C} & -0.839161 & 13.489110 & 33.212856 \\ \mathrm{H} & -2.603770 & 13.453065 & 31.973107 \\ \mathrm{H} & 1.094368 & 13.576145 & 34.166990 \\ \mathrm{H} & -1.380588 & 13.304738 & 34.138599 \\ \mathrm{H} & 7.580617 & 11.632609 & 29.692014 \\ \mathrm{H} & 5.739179 & 11.000080 & 28.142225 \\ \mathrm{C} & 6.545489 & 11.686584 & 30.017189 \\ \mathrm{O} & 5.518805 & 11.335133 & 29.150186 \\ \mathrm{C} & 6.257658 & 12.111989 & 31.324662 \\ \mathrm{C} & 4.204196 & 11.412771 & 29.603132 \\ \mathrm{H} & 3.894399 & 11.845025 & 30.882709 \\ \mathrm{H} & 12610 & 12.538534 & 32.749404 \\ \mathrm{H} & 11.908422 & 31.212462\end{array}$




$\begin{array}{llll}\mathrm{Cl} & 2.893291 & 10.927950 & 28.523963 \\ \mathrm{C} & 7.094792 & 12.816780 & 33.456449 \\ \mathrm{H} & 8.080936 & 13.000171 & 33.886208 \\ \mathrm{H} & 6.586436 & 12.019456 & 34.013449 \\ \mathrm{H} & 6.498271 & 13.737755 & 33.500030\end{array}$

\section{F (Pd(0)-DavePhos complex with Pd-aryl binding with PhMgBr bound and ArF F-bound to $\mathrm{Mg}$ )}

Final Gibbs free enthalpy $\left(\Delta \mathrm{G}=\Delta \mathrm{H}-\mathrm{T}^{*} \Delta \mathrm{S}\right)=-4987.50632340 E_{\mathrm{h}}$

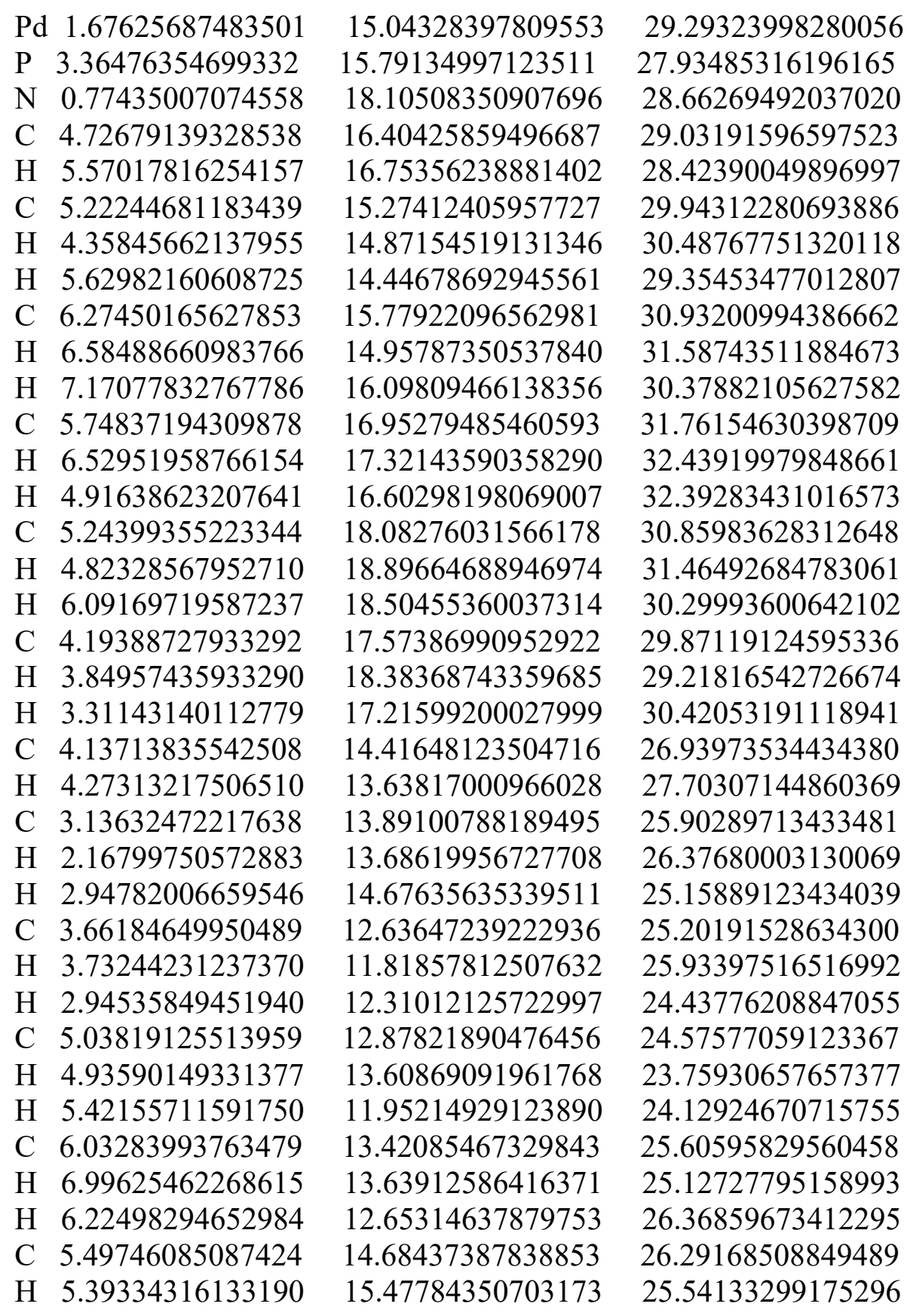




\begin{tabular}{|c|c|c|c|}
\hline & 6.21915246255217 & & \\
\hline $\mathrm{C}$ & & & \\
\hline & & & \\
\hline & & & \\
\hline & 21227200392316 & & \\
\hline & 10391180163083 & & 293 \\
\hline & 98046798584440 & & \\
\hline & 89762572697145 & & 541 \\
\hline & & & \\
\hline & 17 & & \\
\hline & & & \\
\hline & 0.65 & & \\
\hline & & & \\
\hline & 14 & & 24.8 \\
\hline & & & \\
\hline & 777 & & \\
\hline & -1.65 & & \\
\hline $\mathrm{I}$ & -2.5 & & \\
\hline & -1.0 & & \\
\hline$\Pi$ & -1.4 & & \\
\hline & 0.12 & & \\
\hline & & & \\
\hline & & & \\
\hline $\mathrm{H}$ & & & \\
\hline & -0.1 & 2069 & \\
\hline & 0.51 & & \\
\hline $\mathrm{H}$ & & & \\
\hline & -0.4 & 3119 & \\
\hline H & 1.2 & & \\
\hline & & 7873 & \\
\hline & -0.3 & 200 & \\
\hline & 0.44 & & \\
\hline i & & & \\
\hline & 1.04 & & \\
\hline & -1.7 & & \\
\hline & -1.4 & 175 & \\
\hline & 0.30 & & \\
\hline $\mathrm{H}$ & 2.12 & 544 & 32.1 \\
\hline & $-1.0^{\prime}$ & & \\
\hline $\mathrm{H}$ & -2.77 & 4414 & 31.8002 \\
\hline & 0.80 & 3319 & 34.15670 \\
\hline $\mathrm{H}$ & & & \\
\hline $\mathrm{H}$ & 7.21070882448128 & 11.53114734728335 & 29.42957557189877 \\
\hline & 5.15315397143616 & & 28.05943548645782 \\
\hline & 6.22362229216671 & 11.64801083378801 & 29.86694620624588 \\
\hline & 5.08468689254025 & 11.42886114969263 & 29.0998315317183 \\
\hline
\end{tabular}




$\begin{array}{llll}\mathrm{O} & 7.28607403212090 & 12.22017051280342 & 31.87636527455453 \\ \mathrm{C} & 6.10965792884044 & 12.02388974022432 & 31.21595800561075 \\ \mathrm{C} & 3.85406320847473 & 11.59500204564184 & 29.70998431296920 \\ \mathrm{C} & 4.84326436394613 & 12.17661818729515 & 31.79666281262962 \\ \mathrm{C} & 3.69643309240195 & 11.95580060602063 & 31.02985574014900 \\ \mathrm{H} & 4.72919727867144 & 12.47085587353793 & 32.83291652223378 \\ \mathrm{H} & 2.70789210560995 & 12.07315193181295 & 31.46095920338029 \\ \mathrm{~F} & 2.70352515625157 & 11.38682815686299 & 28.93501430781504 \\ \mathrm{C} & 7.23007275060612 & 12.53257236055924 & 33.27388668343610 \\ \mathrm{H} & 8.26901147470200 & 12.60506108277604 & 33.60020897605986 \\ \mathrm{H} & 6.71864420266889 & 11.73845156092246 & 33.83323633552254 \\ \mathrm{H} & 6.72453573815937 & 13.49223619294260 & 33.44684169021494\end{array}$

TS $_{11-12} \mathrm{Cl}$ (Pd(0)-DavePhos complex with Pd-aryl binding with PhMgBr bound and ArCl Cl-bound to $\mathrm{Mg}$, $\mathrm{ArCl} \pi$-bond to Pd TS)

Final Gibbs free enthalpy $\left(\Delta \mathrm{G}=\Delta \mathrm{H}-\mathrm{T}^{*} \Delta \mathrm{S}\right)=-5347.90834634 E_{\mathrm{h}}$

$\begin{array}{llcc}\mathrm{C} & -0.29039369761400 & -0.30748160903922 & -1.45898005829803 \\ \mathrm{C} & 0.28414651103762 & 3.19274161045222 & -0.89395970927255 \\ \mathrm{Pd} & -0.45066278947045 & 1.15825560702791 & 0.19400594313641 \\ \mathrm{Mg} & -1.86873936782522 & 1.18908417414735 & -1.97687886391509 \\ \mathrm{Cl} & -1.02497908996389 & 3.66278212823549 & -2.04959223098259 \\ \mathrm{P} & -0.39146495573551 & 0.33010057955571 & 2.40763865062252 \\ \mathrm{C} & 0.97890908042929 & -0.92856419902327 & 2.47086769244917 \\ \mathrm{H} & 1.19251369303834 & -1.19353104675956 & 3.51410043946361 \\ \mathrm{C} & 2.23617130090353 & -0.28695713820629 & 1.85858176061904 \\ \mathrm{H} & 1.97325052728970 & 0.08294993725982 & 0.85690451259035 \\ \mathrm{H} & 2.54374880753956 & 0.58618590584299 & 2.44534079558528 \\ \mathrm{C} & 3.39584524084620 & -1.28152459080730 & 1.75993988653982 \\ \mathrm{H} & 4.25081355510306 & -0.79810666406126 & 1.26800326610054 \\ \mathrm{H} & 3.72541965937628 & -1.55631360908724 & 2.77360424834923 \\ \mathrm{C} & 2.98788229575110 & -2.54561616628787 & 0.99985237917956 \\ \mathrm{H} & 3.82240525572814 & -3.25830383544092 & 0.97132622487627 \\ \mathrm{H} & 2.74934640555340 & -2.27948638049243 & -0.03870698520699 \\ \mathrm{C} & 1.75487868358826 & -3.19273455965581 & 1.63392019683960 \\ \mathrm{H} & 1.44756675806545 & -4.07308842242923 & 1.05456256516058 \\ \mathrm{H} & 2.00749307684274 & -3.54586017188702 & 2.64570040207857 \\ \mathrm{C} & 0.58812262085421 & -2.20427318049710 & 1.71534769959938 \\ \mathrm{H} & -0.26823580776854 & -2.67999619938257 & 2.20658368146444 \\ \mathrm{H} & 0.27236371554841 & -1.93574008504809 & 0.70148440223479 \\ \mathrm{C} & 0.12540820187065 & 1.55406871191976 & 3.73588608803031 \\ \mathrm{H} & 1.00714281980192 & 2.01774085394338 & 3.27378057245103 \\ \mathrm{C} & -0.93746914589737 & 2.64608083535631 & 3.91034518724043 \\ \mathrm{H} & -1.19594427254837 & 3.07043938815050 & 2.93577643759134 \\ \mathrm{H} & -1.85150875242086 & 2.18950405014239 & 4.31607285664813 \\ \mathrm{C} & -0.47215686025175 & 3.76138812568116 & 4.85293595433143\end{array}$




\begin{tabular}{|c|c|c|c|}
\hline & & & \\
\hline & -1.28010657963551 & & \\
\hline & .01830917148034 & 3.20720076895446 & 6.20435974110805 \\
\hline & .88374551901896 & & 6.72454269699706 \\
\hline & & 4.01739186314279 & \\
\hline & & & 50677 \\
\hline & & & \\
\hline & & & 3014 \\
\hline & & & \\
\hline & 3554 & 53776 & 62514 \\
\hline & 1.3325 & & \\
\hline & -1.83796 & -0.5 & 6746 \\
\hline & & & 722 \\
\hline & -0.667 & & 7110 \\
\hline & -2.735719936 & & \\
\hline & -2.56 & & \\
\hline & -4.024 & -1.7 & 1123 \\
\hline & -4.8 & & \\
\hline & -4.21 & -1.0 & 015 \\
\hline & -5.20 & & \\
\hline & -3.135 & -0.4 & 5448 \\
\hline & & & \\
\hline & -3.320 & & \\
\hline & -2.93 & & \\
\hline & -3.74 & & \\
\hline & -3.65 & 3.5 & 703 \\
\hline & -4.3 & & 331 \\
\hline & -4.40 & 0.4 & 124 \\
\hline $\mathrm{I}$ & -4.83 & -0.0 & 9783 \\
\hline & -3.98 & & 8118 \\
\hline 4 & & & \\
\hline & 1.033 & -0.34 & 7088 \\
\hline C & -0.43 & -2.7 & 6535 \\
\hline 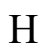 & -2.03 & & 1518 \\
\hline & $1.61^{\prime}$ & -1.51 & -2.4 \\
\hline $\mathrm{H}$ & 1.63 & 0.56 & -1.9 \\
\hline & 0.88 & -2.70 & -2.4 \\
\hline 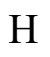 & -1.022 & 89977 & 88538 \\
\hline & 2.650 & -1.49 & 1262 \\
\hline $\mathrm{H}$ & & & \\
\hline & 0.120 & 3.52 & 479891 \\
\hline $\mathrm{H}$ & 1.683 & 2.82 & 20104 \\
\hline & 3.64988167314932 & 2.80008204342422 & -1.02577161442170 \\
\hline & -0.83421902969351 & & \\
\hline $\mathrm{C}$ & 2.67582060787412 & 3.01157195776814 & -0.59998749351978 \\
\hline & 1.26656005730111 & 3.63858934867501 & 1.27357903550497 \\
\hline
\end{tabular}




$\begin{array}{llll}\mathrm{H} & 1.17592342586965 & 3.95431364584661 & 2.30663756520750 \\ \mathrm{C} & 1.56499230941999 & 2.99126351948473 & -1.43378441396149 \\ \mathrm{C} & 2.52633753155973 & 3.33031094073432 & 0.76501566275752 \\ \mathrm{Br} & -2.79124481182906 & 1.03684337997101 & -4.27093856284678 \\ \mathrm{O} & 3.56248528464141 & 3.38050104830434 & 1.65835391856949 \\ \mathrm{C} & 4.85428630183440 & 2.96214386915286 & 1.20692685768713 \\ \mathrm{H} & 5.50408543448794 & 3.01292546215650 & 2.08325615981422 \\ \mathrm{H} & 4.82164691254164 & 1.93102209915592 & 0.82969808662701 \\ \mathrm{H} & 5.23979771071064 & 3.62872440809333 & 0.42395090140388 \\ \mathrm{H} & -4.68799359889837 & 2.36945051311765 & -1.72435902717612 \\ \mathrm{~N} & -4.13214139850918 & -1.75180262692398 & 0.18165881800595 \\ \mathrm{C} & -3.18832477853199 & -2.68057346010809 & 0.79844549654890 \\ \mathrm{H} & -2.88864705606998 & -3.42875076720941 & 0.05464133150256 \\ \mathrm{H} & -2.29065850971879 & -2.15913958021946 & 1.12172369276393 \\ \mathrm{H} & -3.62722235426639 & -3.19898896718729 & 1.66056951747024 \\ \mathrm{C} & -4.99814753294325 & -2.35936029722831 & -0.81582312629973 \\ \mathrm{H} & -5.26316620984105 & -3.36478874104340 & -0.47484246529857 \\ \mathrm{H} & -5.92084035029659 & -1.78213665577699 & -0.92738668628375 \\ \mathrm{H} & -4.51351473483164 & -2.44816247924690 & -1.80305229097915\end{array}$

TS $_{11-12} \mathrm{~F}$ (Pd(0)-DavePhos complex with Pd-aryl binding with PhMgBr bound and ArF Fbound to Mg, ArF $\pi$-bond to Pd TS)

Final Gibbs free enthalpy $\left(\Delta \mathrm{G}=\Delta \mathrm{H}-\mathrm{T}^{*} \Delta \mathrm{S}\right)=-4987.49378802 E_{\mathrm{h}}$

$\begin{array}{llcc}\mathrm{C} & -0.30523269360957 & -0.33174625234631 & -1.48553663504097 \\ \mathrm{C} & 0.22495664026660 & 3.08135379065043 & -0.85836046578907 \\ \mathrm{Pd} & -0.43711720570303 & 1.06164434219831 & 0.19524373109697 \\ \mathrm{Mg} & -1.77421606525569 & 1.31935786035992 & -1.97929739975359 \\ \mathrm{~F} & -0.83299201665370 & 3.28506577748300 & -1.81439132131313 \\ \mathrm{P} & -0.35156384399266 & 0.29987139447588 & 2.42662184565947 \\ \mathrm{C} & 0.98961779650202 & -0.98731619649610 & 2.50474585581239 \\ \mathrm{H} & 1.18776778504412 & -1.26199230724150 & 3.54845381586132 \\ \mathrm{C} & 2.26524652996308 & -0.36805112587971 & 1.90661167183752 \\ \mathrm{H} & 2.01659833863759 & 0.02297235087563 & 0.90915728399611 \\ \mathrm{H} & 2.59053343654516 & 0.48877835262128 & 2.50834447815235 \\ \mathrm{C} & 3.40140315348145 & -1.38828726539908 & 1.79584362702800 \\ \mathrm{H} & 4.26904524712990 & -0.91731665951741 & 1.31435789169431 \\ \mathrm{H} & 3.72053491182353 & -1.68796309556592 & 2.80581077341214 \\ \mathrm{C} & 2.96472442794399 & -2.62974962561260 & 1.01459893825482 \\ \mathrm{H} & 3.78366278898220 & -3.35945369232118 & 0.97102801935039 \\ \mathrm{H} & 2.72807208384917 & -2.34091098323392 & -0.01869512979097 \\ \mathrm{C} & 1.72065315202487 & -3.26020366391066 & 1.64359748619448 \\ \mathrm{H} & 1.39408022790732 & -4.12747112992198 & 1.05516594272333 \\ \mathrm{H} & 1.96899360075510 & -3.62972844336958 & 2.65048381684448 \\ \mathrm{C} & 0.57402550098093 & -2.24976676251025 & 1.73893153164786 \\ \mathrm{H} & -0.28874365278937 & -2.71244073633317 & 2.23133250328741\end{array}$




\begin{tabular}{|c|c|c|c|}
\hline & 0.25829623940908 & -1.96660721894231 & \\
\hline & & & \\
\hline & .02734777531222 & & \\
\hline & .91016875765538 & 2.60621113154609 & 3.92787221777352 \\
\hline & 1.20583976908527 & & \\
\hline & .79843458868207 & 2.11989998758757 & 4.35543369474083 \\
\hline & .45836022219413 & 3.737132551 & 725304 \\
\hline & 0.34539004241774 & 4.308482773 & 5087 \\
\hline & -1.28980158972464 & & 8529 \\
\hline & 0.05831245165987 & $3.19921 \mathrm{C}$ & 657 \\
\hline & & & 5637 \\
\hline & 06 & 4.023124 & 316 \\
\hline & & & \\
\hline & 1.5091574 & $1.7604 \mathrm{C}$ & 58 \\
\hline & & 2.654924 & \\
\hline & 7 & & \\
\hline & 38 & 0.49 & 7772 \\
\hline & 1.49 & 0.29 & \\
\hline & -1.82 & -0.5 & 402 \\
\hline & -1.68 & & 127 \\
\hline & 12 & -1.4 & 592 \\
\hline & & -1.9( & 798 \\
\hline & -2.62 & -2.50 & 3781 \\
\hline & -4.0 & -1.73 & 353 \\
\hline & -4.9 & & 871 \\
\hline & -4.20 & -0.99 & 708 \\
\hline & & & 487 \\
\hline & -3.1 & -0.38 & 756 \\
\hline & -3.35 & & 460 \\
\hline & $-3.2^{\prime}$ & & 007 \\
\hline & & & \\
\hline & -3.68 & 2.467 & \\
\hline I & -3.6 & 3.550 & \\
\hline & -4.24 & 1.7997 & 011 \\
\hline C & -4.34 & & \\
\hline $\mathrm{H}$ & -4.76 & -0.08869 & 8530 \\
\hline & -3.93 & -0.37230 & \\
\hline $\mathrm{C}$ & -1.086810 & -1.50334384 & 2263 \\
\hline & 1.025122 & -0.429288175 & -1.95 \\
\hline $\mathrm{C}$ & & & -2.162 \\
\hline & -2.12516113990253 & -1.49022 & 57802 \\
\hline $\mathrm{C}$ & 1.548403 & -1.612061462 & -2.4772 \\
\hline & 1.67940589064092 & 0.43760843727301 & -1.90017671250399 \\
\hline & 0.75009492812861 & -2.75563271015476 & -2.5743 \\
\hline & -1.22289639414413 & -3.56478907875113 & -2.24460769012828 \\
\hline & 2.58795646456227 & -1.64820848980854 & -2.80051821340483 \\
\hline
\end{tabular}




$\begin{array}{llcc}\mathrm{H} & 1.15765768733112 & -3.67945061829563 & -2.97971028172642 \\ \mathrm{C} & 0.01944187823972 & 3.56888904146401 & 0.44697465656899 \\ \mathrm{H} & 1.63090088080843 & 2.63922125326797 & -2.42977078818794 \\ \mathrm{H} & 3.59169434576354 & 2.81645747693405 & -0.93262033276194 \\ \mathrm{H} & -0.96373957169778 & 3.90026204217262 & 0.75993481235298 \\ \mathrm{C} & 2.60384970005114 & 3.02347648400900 & -0.53789088666827 \\ \mathrm{C} & 1.14297822703499 & 3.76025996878228 & 1.25764115883171 \\ \mathrm{H} & 1.02700124251677 & 4.15310121021968 & 2.26152844599636 \\ \mathrm{C} & 1.50614393560618 & 2.90320403298667 & -1.38463210519978 \\ \mathrm{C} & 2.42208570514676 & 3.44548845269251 & 0.79272810801931 \\ \mathrm{Br} & -2.59326951065501 & 1.47264313310241 & -4.30216232368122 \\ \mathrm{O} & 3.43857254939563 & 3.58569736074316 & 1.69976896204567 \\ \mathrm{C} & 4.74692647713675 & 3.16587286371105 & 1.30284179359483 \\ \mathrm{H} & 5.37984962383961 & 3.29720620521075 & 2.18317711505322 \\ \mathrm{H} & 4.74424280192111 & 2.10896877451985 & 1.00261857965902 \\ \mathrm{H} & 5.13196945223423 & 3.78108023988324 & 0.47839289868376 \\ \mathrm{H} & -4.62209435632319 & 2.35452345672284 & -1.77905171325270 \\ \mathrm{~N} & -4.09162590247279 & -1.74583612669500 & 0.17918930921216 \\ \mathrm{C} & -3.13149926859448 & -2.66357342641179 & 0.78630349056862 \\ \mathrm{H} & -2.83243155771144 & -3.41095729628453 & 0.04139727941314 \\ \mathrm{H} & -2.23549811854765 & -2.13000539479438 & 1.09317029530882 \\ \mathrm{H} & -3.55371319329081 & -3.18241373052782 & 1.65649019103087 \\ \mathrm{C} & -4.96923933889083 & -2.36389288809830 & -0.80056938615242 \\ \mathrm{H} & -5.24166659629951 & -3.36002612399514 & -0.43816384036531 \\ \mathrm{H} & -5.88652773872464 & -1.77925481916569 & -0.91762734402717 \\ \mathrm{H} & -4.49269718724730 & -2.47829658673391 & -1.78935029664360\end{array}$

12Cl (Pd(0)-DavePhos complex with Pd-aryl binding with PhMgBr bound and 4methoxychlorobenzene bound)

Final Gibbs free enthalpy $\left(\Delta \mathrm{G}=\Delta \mathrm{H}-\mathrm{T}^{*} \Delta \mathrm{S}\right)=-5347.90569828 E_{\mathrm{h}}$

$\begin{array}{cccc}\mathrm{C} & -0.31990131797248 & -0.34352673220413 & -1.37555121906192 \\ \mathrm{C} & 0.27358479258611 & 3.06986788817845 & -0.85105550063051 \\ \mathrm{Pd} & -0.41384302136134 & 1.25993406352202 & 0.18493474026683 \\ \mathrm{Mg} & -1.79074484064888 & 1.23093328821693 & -2.01657877557484 \\ \mathrm{Cl} & -0.96467346084978 & 3.66564020011305 & -2.10445795674786 \\ \mathrm{P} & -0.35686686486123 & 0.30045595448164 & 2.36277425220276 \\ \mathrm{C} & 1.01760396038751 & -0.94750257875411 & 2.43278331630431 \\ \mathrm{H} & 1.20351502016218 & -1.23656502201755 & 3.47516068507837 \\ \mathrm{C} & 2.28937604479727 & -0.28645434108518 & 1.87422629514758 \\ \mathrm{H} & 2.05911432320972 & 0.10067246046480 & 0.87154399395652 \\ \mathrm{H} & 2.57543731518024 & 0.57793737771382 & 2.48428775179281 \\ \mathrm{C} & 3.45581897372654 & -1.27528218775085 & 1.80125298336380 \\ \mathrm{H} & 4.32524133089235 & -0.78224192103500 & 1.34642841766886 \\ \mathrm{H} & 3.75029881467484 & -1.56122880255437 & 2.82203562607031 \\ \mathrm{C} & 3.08453325359536 & -2.53236179586927 & 1.01135260573298\end{array}$




\begin{tabular}{|c|c|c|c|}
\hline & 92252209786301 & -3.24166851878357 & \\
\hline & & & \\
\hline & & -3.19157145900807 & \\
\hline & & & 97961873349058 \\
\hline & 04000479491606 & & 2.59930642639601 \\
\hline & 65894749512280 & & 1.63346812607072 \\
\hline & .22335124171001 & -2.68599561039772 & 2.07024339298709 \\
\hline & 39646004840294 & -1.91078826098030 & \\
\hline & & & \\
\hline & 152695 & 2.03 & 602 \\
\hline & & 071 & 894 \\
\hline & 923442 & 909 & 2657 \\
\hline & & 898 & \\
\hline & -0.49444 & 3.6977 & 3217 \\
\hline & 0.31619336158707 & & \\
\hline & -1.3231 & & 78 \\
\hline & 0.00763 & 3.135 & \\
\hline & 00 & & 594 \\
\hline & 2628 & 3.946 & \\
\hline & & & \\
\hline & 40 & & \\
\hline & & & \\
\hline & 0.62424 & & \\
\hline & -0.20389 & 0.45 & 66 \\
\hline & & & \\
\hline & 278389 & -0.545 & 790 \\
\hline & & & \\
\hline & -0.6 & -1.5 & \\
\hline & 93719 & 4424 & \\
\hline & -2.5 & & \\
\hline C & & & \\
\hline & 405362 & -2.200 & \\
\hline & $-4.1937012^{\prime}$ & -1.004 & \\
\hline & -5.15 & -0.88 & \\
\hline & -3.11 & -0.412 & \\
\hline & 193992 & 0.3018 & \\
\hline & -3.2501 & 1.6868 & \\
\hline & -2.854608 & 2.1874 & 357 \\
\hline & -3.64651974013995 & 2.4515 & \\
\hline $\mathrm{H}$ & & & \\
\hline & -4.23324432998239 & 1.79744121292895 & 65948 \\
\hline $\mathrm{C}$ & -4.37932163594733 & 0.4045 & -0.9 \\
\hline & -4.80872088638622 & -0.07300282784689 & -1.79970908810989 \\
\hline & -3.99581699868490 & -0.380540 & \\
\hline & -1.08477473898068 & -1.53074095692071 & -1.43194641305617 \\
\hline & 0.99203935205382 & -0.43857402740878 & -1.88986062590234 \\
\hline
\end{tabular}




$\begin{array}{llcc}\mathrm{C} & -0.58183287426880 & -2.72939298280022 & -1.94339951810646 \\ \mathrm{H} & -2.10889289020574 & -1.52096579542222 & -1.07324500316108 \\ \mathrm{C} & 1.51455104292174 & -1.63234504284331 & -2.38869058915661 \\ \mathrm{H} & 1.63085516866722 & 0.44192397110950 & -1.89286389893235 \\ \mathrm{C} & 0.73119236732927 & -2.78943128122833 & -2.41133014237406 \\ \mathrm{H} & -1.21090974374654 & -3.61828849584294 & -1.96801019522732 \\ \mathrm{H} & 2.53883487048598 & -1.66486949881782 & -2.75637339639964 \\ \mathrm{H} & 1.13657494009867 & -3.72111484152069 & -2.79989539047576 \\ \mathrm{C} & 0.08668481436306 & 3.50650462913714 & 0.49956650048086 \\ \mathrm{H} & 1.73121680597772 & 2.66862411511410 & -2.40691843273957 \\ \mathrm{H} & 3.66494228862096 & 2.83069591901686 & -0.91331274601012 \\ \mathrm{H} & -0.86769949220051 & 3.91828595845754 & 0.80617392080435 \\ \mathrm{C} & 2.67663591440283 & 3.03024595557420 & -0.51441957427438 \\ \mathrm{C} & 1.22575635826117 & 3.72446871161602 & 1.30110363825480 \\ \mathrm{H} & 1.10985762710844 & 4.12217363636309 & 2.30360199889525 \\ \mathrm{C} & 1.58696594996125 & 2.90051007257694 & -1.35608631108372 \\ \mathrm{C} & 2.49743024607929 & 3.43642298283437 & 0.82942397573532 \\ \mathrm{Br} & -2.67262569249496 & 0.97535335425638 & -4.31328078267914 \\ \mathrm{O} & 3.52819494935610 & 3.59877329515982 & 1.72139133322787 \\ \mathrm{C} & 4.84410157976710 & 3.23989844204997 & 1.29429499640339 \\ \mathrm{H} & 5.49123547056894 & 3.40510991696865 & 2.15900015182846 \\ \mathrm{H} & 4.88761521455539 & 2.18305284112576 & 0.99753730335196 \\ \mathrm{H} & 5.18126964933282 & 3.86878490102230 & 0.45922542355482 \\ \mathrm{H} & -4.58549036895921 & 2.35808605995484 & -1.80135927992043 \\ \mathrm{~N} & -4.23178283764482 & -1.74435673603723 & 0.17931640255987 \\ \mathrm{C} & -3.35821678551926 & -2.73140678572361 & 0.80804074802062 \\ \mathrm{H} & -3.07059531982392 & -3.48119275127369 & 0.05997017235763 \\ \mathrm{H} & -2.44801228209411 & -2.26784054143986 & 1.17958899874029 \\ \mathrm{H} & -3.85744280000687 & -3.24116977799377 & 1.64215787271873 \\ \mathrm{C} & -5.13228025553020 & -2.30965124137066 & -0.81266925552177 \\ \mathrm{H} & -5.45814806148287 & -3.29224791661570 & -0.45985142376898 \\ \mathrm{H} & -6.01707429114629 & -1.67836129789937 & -0.93367379038628 \\ \mathrm{H} & -4.65244492006976 & -2.44043013706233 & -1.79738722360279\end{array}$

\section{$12 F$ (Pd(0)-DavePhos complex with Pd-aryl binding with $\mathrm{PhMgBr}$ bound and 4- methoxyfluorobenzene bound)}

Final Gibbs free enthalpy $\left(\Delta \mathrm{G}=\Delta \mathrm{H}-\mathrm{T}^{*} \Delta \mathrm{S}\right)=-4987.49401488 E_{\mathrm{h}}$

$\begin{array}{cccc}\text { C } & -0.27650111618963 & -0.34083100561946 & -1.39168582437802 \\ \text { C } & 0.18283181522440 & 3.01271834078649 & -0.86135613016646 \\ \text { Pd } & -0.40415920713834 & 1.18563277591669 & 0.21324183668319 \\ \mathrm{Mg} & -1.70194686668592 & 1.30929365497760 & -2.00902652682802 \\ \text { F } & -0.85328641092963 & 3.27087672835343 & -1.87581267702508 \\ \text { P } & -0.32632541449892 & 0.25792981160432 & 2.39341215151873 \\ \text { C } & 0.97904294201725 & -1.06005411421386 & 2.47773433984076 \\ \text { H } & 1.16383670603209 & -1.33818248531711 & 3.52327689356137\end{array}$




\begin{tabular}{|c|c|c|c|}
\hline & 2.27565958678852 & -0.47886895335482 & \\
\hline & .05090964555064 & & \\
\hline & 61558367123903 & & 2.47612040423938 \\
\hline & 38545122500137 & -1.53099798624621 & 1.81123114480982 \\
\hline & 27383563036294 & -1.09213389882683 & 1.33864911309746 \\
\hline & 67790838357935 & -1.82345775749723 & 2.83078044004922 \\
\hline & & -2.77246961430690 & 1.03950138 \\
\hline & 72814496505773 & -3.52657582 & 996140 \\
\hline & 26685732 & -2.49393 & 089400 \\
\hline & 65342992289970 & -3.35768 & 56959892 \\
\hline & & -4.2 & 1673 \\
\hline & 09536 & -3.7 & 54380 \\
\hline & 468 & -2.3 & 6871 \\
\hline & 8740 & -2.7434875 & 491422 \\
\hline & 0.25224048667874 & -2.02617512568440 & 0.69328922559867 \\
\hline & 0.23078 & 1.4 & 0668 \\
\hline & 1.04665 & 2.0 & 0641 \\
\hline C & $-0.8^{\prime}$ & & 45694 \\
\hline & -1.20 & 506 & 06840 \\
\hline H & -1.7 & 492 & 60370 \\
\hline & -0.418 & 0606 & 67028 \\
\hline $\mathrm{H}$ & 0.3580 & & \\
\hline $\mathrm{H}$ & -1.25855 & 7117 & 03563 \\
\hline & 0.150412 & 3.0 & 4857 \\
\hline $\mathrm{H}$ & -0.6516 & & 2029 \\
\hline & 0.517568 & 3.90 & 4003 \\
\hline $\mathrm{C}$ & & & 7264 \\
\hline & 1.6601 & & 2000 \\
\hline H & 2.10723 & & 7985 \\
\hline & 0.78534 & & \\
\hline $\mathrm{H}$ & & & \\
\hline $\mathrm{H}$ & 1.607689 & & 0958 \\
\hline $\mathrm{C}$ & -1.82103020378669 & -0.5 & 36912 \\
\hline $\mathrm{C}$ & -1.7005 & -1.2 & 57390 \\
\hline $\mathrm{H}$ & -0.7187 & & \\
\hline $\mathrm{C}$ & -2.8083481713254 & -1.83 & 315029 \\
\hline $\mathrm{H}$ & -2.67968461993610 & & \\
\hline $\mathrm{C}$ & -4.07974787587069 & -1.632776 & 15543 \\
\hline $\mathrm{H}$ & -4.95866155062188 & -2.045653542076 & 68896634 \\
\hline $\mathrm{C}$ & & & \\
\hline $\mathrm{H}$ & -5.19984337341854 & 712981 & 4030546 \\
\hline $\mathrm{C}$ & -3.10468541304524 & -0.35895 & 333953 \\
\hline $\mathrm{C}$ & -3.37430673207962 & 0.33483058035455 & 1.28198055732794 \\
\hline $\mathrm{C}$ & -3.21550998746355 & & \\
\hline $\mathrm{H}$ & -2.83624461907770 & 2.23423501421372 & 2.08260665842695 \\
\hline & -3.60972050133278 & 2.46822251948589 & 0.09257769111974 \\
\hline
\end{tabular}




\begin{tabular}{|c|c|c|c|}
\hline & -3.50959237034004 & & \\
\hline & -4.17853555806942 & & -0.99301381755735 \\
\hline & -4.30716028804187 & 0.40175771550446 & -0.96959937043034 \\
\hline & -4.72411886360961 & & \\
\hline & -3.92825252008906 & & \\
\hline & -1.05422183741550 & & 4899 \\
\hline & 1.04974394433886 & -0.43612731122685 & -1.8687708 \\
\hline & -0.55031128927007 & 9253 & -2.044161 \\
\hline & -2.088 & & -1. \\
\hline & 1.5718 & -1.61 & -2.3 \\
\hline & 1.69 & & \\
\hline & 0.77 & -2.7 & -2.4 \\
\hline & -1.1 & & 7767 \\
\hline & 2.606 & -1.6 & -2.7 \\
\hline & & & \\
\hline & -0.06 & & 767 \\
\hline & 1.65 & 2.6 & -2.4 \\
\hline & 3.56 & & -0.9 \\
\hline & -1.0 & & 821 \\
\hline & 2.56 & & -0.5 \\
\hline & 1.04 & & \\
\hline & 0.89 & & \\
\hline & 1.49 & & \\
\hline C & & & \\
\hline & -2.4 & & 681 \\
\hline ) & 3.35 & & \\
\hline & 4.69 & & \\
\hline & 5.31 & & \\
\hline & 4.80 & 2.53 & 1.0 \\
\hline & 5.00 & & \\
\hline & -4.5 & & 932 \\
\hline & $-4.1<$ & 5693 & 545 \\
\hline & -3.2 & -2.68 & \\
\hline & -2.95 & 83501 & 9008 \\
\hline & -2.3 & & \\
\hline 11 & -3.72421104466689 & -3.17997120734632 & 2083356 \\
\hline & -5.03 & -2.32636876 & \\
\hline & -5.34713266147543 & -3.30595259705848 & -0.44499312345724 \\
\hline & & -1.70841640511019 & -0.95339431113435 \\
\hline & -4.54617496411153 & -2.47128830393561 & -1.79563226998923 \\
\hline
\end{tabular}

\section{TS ${ }_{12-8} \mathrm{Cl}$ (Pd(0)-DavePhos complex with Pd-aryl binding with PhMgBr bound and 4- methoxychlorobenzene TS)}

Final Gibbs free enthalpy $\left(\Delta \mathrm{G}=\Delta \mathrm{H}-\mathrm{T}^{*} \Delta \mathrm{S}\right)=-5347.90536196 E_{\mathrm{h}}$

$\begin{array}{llll}\text { C } & -0.14275983695478 & -0.36849801752285 & -1.20193816834248\end{array}$ 


\begin{tabular}{|c|c|c|c|}
\hline & 0.46970800263629 & & \\
\hline & -0.30996419908810 & 1.25583276860394 & 0.26772114604104 \\
\hline & -1.40384729767923 & 1.28868648040523 & \\
\hline & -0.30392655492851 & 0.22283005902063 & 2.41385678966424 \\
\hline & 0.97223426666266 & & \\
\hline & 1.11559426394436 & & 1916304148 \\
\hline & 2.3001139 & -0.5656 & \\
\hline & 2.1293731 & & \\
\hline & 2.6243 & & \\
\hline & 7396729411 & -1.635 & 1.99 \\
\hline & & & 944 \\
\hline & 3.629 & -1.90 & 3271 \\
\hline & 493 & -2.89 & 666 \\
\hline & 3.742 & -3.659 & 957 \\
\hline & 2.82398792537729 & & \\
\hline & 1.63 & -3.4 & \\
\hline & 1.313 & -4.30 & 1.20 \\
\hline & & $-3.7 \varepsilon$ & \\
\hline & 0.54 & -2.37 & 1.77( \\
\hline I & -0.3 & -2.77 & 2.20 \\
\hline & $0.31^{\circ}$ & -2.1005 & 0.73 \\
\hline & 0.245 & & \\
\hline & $1.06 ?$ & & \\
\hline & -0.86 & 2.48 & 116 \\
\hline I & -1.17 & & 09 \\
\hline & -1.73 & 1.962 & 2211 \\
\hline C & -0.4 & & 226 \\
\hline & 0.36 & 4.18 & 4.49 \\
\hline $\mathrm{H}$ & -1.26 & 81 & 986 \\
\hline & 0.14 & 2.9940 & \\
\hline $\mathrm{H}$ & & & \\
\hline & 0.49 & & \\
\hline & 1.26 & 2.0058 & \\
\hline & 1.65 & & \\
\hline & 2.10 & & \\
\hline & 0.790 & 0.886 & 5.03 \\
\hline & 0.003 & & \\
\hline $\mathrm{H}$ & 5724311678 & 0.19467 & 4.8447 \\
\hline & 0323792 & -0.4997 & \\
\hline $\mathrm{C}$ & -1.75 & -1.2686 & 4.31 \\
\hline $\mathrm{H}$ & 112795 & -1.4779 & 889934 \\
\hline $\mathrm{C}$ & -2.88 & -1.7858 & 4.9264 \\
\hline 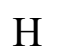 & -2.79289972115885 & -2.37354342630007 & 5.83621279811902 \\
\hline & -4.14605378048138 & -1.53809933 & \\
\hline $\mathrm{H}$ & -5.04489612033928 & -1.91715734658073 & 4.85198636274792 \\
\hline & -4.24267702510430 & -0.81557803721199 & 3.18795933488017 \\
\hline
\end{tabular}




\begin{tabular}{|c|c|c|c|}
\hline & -5.21572721959588 & -0.65261593090030 & \\
\hline & -3.10421621285317 & -0.30516761018174 & \\
\hline & -3.32215069469769 & & .24365771072644 \\
\hline & -3.08493393778536 & & \\
\hline & -2.71587700830239 & 2.27116334430578 & \\
\hline & -3.38713450832306 & 2.51983427072893 & 977037227 \\
\hline & -3.21053921814944 & & \\
\hline & -3.95199587222688 & 1.86649732274513 & \\
\hline & -4.1774 & & \\
\hline & 5770486941 & 0.00802 & 6900 \\
\hline & -3.88 & & \\
\hline & -0.98 & -1.49 & 7924 \\
\hline & 1.174 & 05131 & \\
\hline & -0.540 & -2.68804 & 4181 \\
\hline & -2.01265665269997 & & \\
\hline & 1.63 & -1.70 & \\
\hline & 1.86 & 0.32 & -1.6 \\
\hline & 0.77 & -2.80 & \\
\hline & -1.22 & -3.5 & 7123 \\
\hline & 2.66 & -1.78 & \\
\hline & 1.13 & -3.73 & -2.7 \\
\hline & 0.13 & & \\
\hline & 2.08 & & \\
\hline & 3.85 & 2.97 & \\
\hline & -0.8 & & 093 \\
\hline & 2.82 & 3.148 & -0.1 \\
\hline & & & \\
\hline & 0.93 & 4.40 & \\
\hline & 1.82 & 2.84 & -1.0 \\
\hline & 2.50 & 3.67 & \\
\hline & & & \\
\hline $\mathrm{O}$ & 3.44 & 4.00 & \\
\hline & 4.81 & 3.742 & \\
\hline & 5.37 & & \\
\hline & 4.98 & & \\
\hline & 5.15 & 4.31768 & 587 \\
\hline & -4.22 & & \\
\hline & -4.18 & -1.6377 & 4917 \\
\hline & -3.3 & -2.6472 & \\
\hline $\mathrm{H}$ & & & \\
\hline & -2.45 & -2.21525 & 06832211 \\
\hline & -3.91861866 & -3.11710 & 52447062 \\
\hline 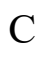 & -5.08051256716333 & -2.18390312423500 & 5546571512 \\
\hline & -5.47522356897173 & -3.13490 & \\
\hline & -5.92195331350114 & -1.50780674197592 & -1.05475197032453 \\
\hline & -4.57388175774761 & -2.37566961705941 & -1.84096024152627 \\
\hline
\end{tabular}


Cl $-0.68037468610916 \quad 3.64560635133895 \quad-2.22814777529338$

\section{TS $_{12-8}$ F (Pd(0)-DavePhos complex with Pd-aryl binding with PhMgBr bound and 4- methoxyfluorobenzene TS)}

Final Gibbs free enthalpy $\left(\Delta \mathrm{G}=\Delta \mathrm{H}-\mathrm{T}^{*} \Delta \mathrm{S}\right)=-4987.49221887 E_{\mathrm{h}}$

\begin{tabular}{|c|c|c|c|}
\hline & -0.21530042885425 & -0.39914805571264 & -1.25757715407221 \\
\hline & 0.26078399681850 & 2.86121702397044 & -0.73101678696266 \\
\hline & -0.37614391565902 & 1.14627182241862 & 0.25117431078458 \\
\hline & -1.55966980161108 & 1.30813142459271 & -2.06942964760458 \\
\hline $\mathrm{F}$ & -0.74471976747981 & 3.11997856784966 & -2.05536172908125 \\
\hline $\mathrm{P}$ & -0.30622646679012 & 0.10068169833759 & 2.38958506862511 \\
\hline $\mathrm{C}$ & 0.94890955880224 & -1.26235560095681 & 2.47617468847839 \\
\hline $\mathrm{H}$ & 1.10812627267919 & -1.55047124018253 & 3.52333114002567 \\
\hline $\mathrm{C}$ & 2.27687183845921 & -0.73016475269126 & 1.90917575992843 \\
\hline $\mathrm{H}$ & 2.08829226566767 & -0.33731190519379 & 0.90167789744821 \\
\hline $\mathrm{H}$ & 2.64194565771700 & 0.10948429315503 & 2.51225923040625 \\
\hline $\mathrm{C}$ & 3.34395689453073 & -1.82666492350206 & 1.84477165046002 \\
\hline $\mathrm{H}$ & 4.25729251219553 & -1.42174834804638 & 1.39100308915910 \\
\hline $\mathrm{H}$ & 3.60607734479476 & -2.13720146930958 & 2.86712396844372 \\
\hline $\mathrm{C}$ & 2.85082063049791 & -3.04373184558309 & 1.05756080280257 \\
\hline $\mathrm{H}$ & 3.61804082007958 & -3.82885032246968 & 1.05078254459767 \\
\hline $\mathrm{H}$ & 2.67578845191502 & -2.75038676960271 & 0.01332277724930 \\
\hline $\mathrm{C}$ & 1.54434887422518 & -3.58162777126108 & 1.64557782360528 \\
\hline $\mathrm{H}$ & 1.17718042727012 & -4.42829014626517 & 1.05159239438188 \\
\hline $\mathrm{H}$ & 1.73253777696414 & -3.95982123996416 & 2.66162728389780 \\
\hline $\mathrm{C}$ & 0.46755362757294 & -2.49450905107558 & 1.70021644913040 \\
\hline $\mathrm{H}$ & -0.43956875921696 & -2.89306929018247 & 2.16705674764376 \\
\hline $\mathrm{H}$ & 0.20490943707082 & -2.19626065628238 & 0.68056233200483 \\
\hline $\mathrm{C}$ & 0.30500722775047 & 1.34431300227011 & 3.65901409301333 \\
\hline $\mathrm{H}$ & 1.14797768051350 & 1.81828465032213 & 3.13610754856738 \\
\hline $\mathrm{C}$ & -0.74798018791826 & 2.43331353548841 & 3.90650771361150 \\
\hline $\mathrm{H}$ & -1.05775524373821 & 2.86623698514025 & 2.95218161515068 \\
\hline $\mathrm{H}$ & -1.63546803250655 & 1.97535811295051 & 4.36325483679025 \\
\hline $\mathrm{C}$ & -0.21851817615051 & 3.53996642598952 & 4.82398821121266 \\
\hline $\mathrm{H}$ & 0.59946476249218 & 4.06721057868392 & 4.31212877217110 \\
\hline $\mathrm{H}$ & -1.01059147252536 & 4.27726289266861 & 5.00619293339262 \\
\hline $\mathrm{C}$ & 0.30582511503339 & 2.97685115423460 & 6.14582448653041 \\
\hline $\mathrm{H}$ & -0.52975535826929 & 2.53363696910380 & 6.70802268828430 \\
\hline $\mathrm{H}$ & 0.71949381491960 & 3.78286370352553 & 6.76552988075610 \\
\hline $\mathrm{C}$ & 1.36431508859816 & 1.90224472081503 & 5.89025502767221 \\
\hline $\mathrm{H}$ & 1.71573623200561 & 1.47429971336772 & 6.83758123950554 \\
\hline $\mathrm{H}$ & 2.23647441101126 & 2.36252808816333 & 5.40289206921390 \\
\hline $\mathrm{C}$ & 0.81807241247201 & 0.78470766205646 & 4.99239669722377 \\
\hline $\mathrm{H}$ & -0.00574923749746 & 0.28619170778804 & 5.51785999713168 \\
\hline $\mathrm{H}$ & 1.59757530986363 & 0.03519781675764 & 4.82547951263766 \\
\hline
\end{tabular}




\begin{tabular}{|c|c|c|c|}
\hline & -1.82678426043422 & & \\
\hline & -1.72956459714155 & -1.37550662813960 & \\
\hline & -0.75344359272362 & -1.59043588675044 & \\
\hline & & & \\
\hline & -2.74545592435690 & & \\
\hline & -4.12025182889318 & & \\
\hline & -5.01177773941348 & -2.02972527002956 & \\
\hline & -4.2347211254 & & 3.2395 \\
\hline & -5.2 & -0.7 & \\
\hline & -3.10 & -0.3 & \\
\hline & -3.3 & & \\
\hline & -3.16 & & 894 \\
\hline & -2.79 & & 867 \\
\hline & -3.53 & 2.4 & 276 \\
\hline & -3.4 & & \\
\hline$y$ & -4.08 & 1.8 & 100 \\
\hline & -4.2545 & & \\
\hline I & -4.6 & & \\
\hline & -3.91 & -0.3 & 457 \\
\hline & -1.06 & & \\
\hline & 1.09 & -0.5 & -1.7 \\
\hline & -0.6 & & \\
\hline & -2.0894 & & 8769 \\
\hline C & 1.52589 & & \\
\hline I & 1.7943 & & \\
\hline & 0.6580 & $-2.7 \xi$ & -2.5 \\
\hline $\mathrm{H}$ & -1.3 & & 590 \\
\hline & 2.54 & $-1.7^{\prime}$ & -2.7 \\
\hline H & 0.9949 & -3.70 & -3.0 \\
\hline & -0.1207 & & 444 \\
\hline & 1.87 & & 992 \\
\hline & 3.61 & & -0.6 \\
\hline $\mathrm{H}$ & & & \\
\hline & 2.58 & & -0.3 \\
\hline & 0.88 & & \\
\hline & 0.609 & & 844 \\
\hline & 1.6066 & & -1.1 \\
\hline $\mathrm{C}$ & 2.22458 & 4.05 & 0.86 \\
\hline & -2.4842 & & \\
\hline $\mathrm{O}$ & 3.1287989 & & \\
\hline & 4.51831881722268 & 4.49 & 1.42623580411752 \\
\hline & 5.04960 & & 2.24923 \\
\hline & 4.81593729512458 & 3.43 & 1.36670546077175 \\
\hline & 4.76810883337557 & 4.996 & \\
\hline & -4.40456119579325 & 2.36896567000348 & -1.85933032464694 \\
\hline & -4.19225049155037 & -1.71981255310340 & 0.16442593997818 \\
\hline
\end{tabular}




$\begin{array}{lllr}\mathrm{C} & -3.34557406188423 & -2.71875288234067 & 0.80932364938674 \\ \mathrm{H} & -3.06610576820087 & -3.48159294170082 & 0.07129245022603 \\ \mathrm{H} & -2.42944276816140 & -2.26997473741829 & 1.18277399035003 \\ \mathrm{H} & -3.86078247265826 & -3.21007606906338 & 1.64501422380652 \\ \mathrm{C} & -5.09232977820359 & -2.27219308113250 & -0.83439859209447 \\ \mathrm{H} & -5.45932812139213 & -3.23621467011531 & -0.46990633144809 \\ \mathrm{H} & -5.95117544643171 & -1.61230441073374 & -0.98493211311623 \\ \mathrm{H} & -4.59947386927901 & -2.43974984747723 & -1.80721806054437\end{array}$

12F-oBn (Pd(0)-Cy ${ }_{2} \mathrm{P}\left(o-B n-\mathrm{C}_{6} \mathrm{H}_{4}\right)$ complex with $\mathrm{Pd}-\mathrm{aryl}$ binding with $\mathrm{PhMgBr}$ bound and 4-methoxyfluorobenzene)

Final Gibbs free enthalpy $\left(\Delta \mathrm{G}=\Delta \mathrm{H}-\mathrm{T}^{*} \Delta \mathrm{S}\right)=-4892.90352205 E_{\mathrm{h}}$

$\begin{array}{cccc}\mathrm{C} & -0.53640785717976 & -0.97444688042871 & -1.24653565832509 \\ \mathrm{C} & -0.18232618408591 & 2.52030932725253 & -1.14783308424867 \\ \mathrm{Pd} & -0.68796122326599 & 0.79632254808177 & 0.07956619942699 \\ \mathrm{Mg} & -1.79558367148831 & 0.57394956654133 & -2.26996385555685 \\ \mathrm{~F} & -1.20613593054066 & 2.66568514998842 & -2.19418003593954 \\ \mathrm{P} & -0.42058046876582 & 0.08175393704626 & 2.34114291864922 \\ \mathrm{C} & 1.12254588905746 & -0.96002762291328 & 2.42964316416849 \\ \mathrm{H} & 1.32406039977430 & -1.22269496058666 & 3.47575070021300 \\ \mathrm{C} & 2.30187542933413 & -0.11598809886304 & 1.90982757724628 \\ \mathrm{H} & 2.06167658837767 & 0.22478194912392 & 0.89380678055359 \\ \mathrm{H} & 2.43108720867662 & 0.78380380023646 & 2.51937252984175 \\ \mathrm{C} & 3.60714713516324 & -0.91737583723286 & 1.90037807247933 \\ \mathrm{H} & 4.41021480834155 & -0.30243327781444 & 1.47268410995421 \\ \mathrm{H} & 3.89760466113164 & -1.14254109186174 & 2.93746224814444 \\ \mathrm{C} & 3.46624542963295 & -2.22626026178088 & 1.11947652959503 \\ \mathrm{H} & 4.40338008244933 & -2.79636020703431 & 1.16509470576036 \\ \mathrm{H} & 3.27403236158273 & -1.99525724658951 & 0.06230059143395 \\ \mathrm{C} & 2.29990133517264 & -3.05955019225341 & 1.65544585436709 \\ \mathrm{H} & 2.18004587567114 & -3.97288038363886 & 1.05981988375098 \\ \mathrm{H} & 2.51630780377076 & -3.37275943797237 & 2.68822140068691 \\ \mathrm{C} & 1.00080822148165 & -2.25292995122229 & 1.61635125641301 \\ \mathrm{H} & 0.15869070187286 & -2.85868481463024 & 1.96600124073603 \\ \mathrm{H} & 0.78774328748615 & -1.98768087562813 & 0.58158090086007 \\ \mathrm{C} & -0.03486499399425 & 1.40823040704515 & 3.61310861116121 \\ \mathrm{H} & 0.80263385597930 & 1.94689114918682 & 3.14852418720942 \\ \mathrm{C} & -1.17449767037135 & 2.41768439722539 & 3.78548120424852 \\ \mathrm{H} & -1.47774939615996 & 2.80984306460502 & 2.80958797863149 \\ \mathrm{H} & -2.04517494948963 & 1.91030583132896 & 4.22148464458202 \\ \mathrm{C} & -0.76297955486266 & 3.57410122118488 & 4.70192032418018 \\ \mathrm{H} & 0.05649177666787 & 4.13323425505637 & 4.22596896964706 \\ \mathrm{H} & -1.60237701497290 & 4.27163992314495 & 4.81747909514412 \\ \mathrm{C} & -0.29729578769574 & 3.06471584869799 & 6.06715130636440 \\ \mathrm{H} & -1.14723611714236 & 2.59697464041480 & 6.58632887632102\end{array}$




\begin{tabular}{|c|c|c|c|}
\hline & & & \\
\hline & & & \\
\hline & 1.11490166510275 & & \\
\hline & 1.71269108377047 & & \\
\hline & 0.41520003111049 & 0.88519509194874 & 4.98229482102602 \\
\hline & & 0.32067349082427 & 019062 \\
\hline & 1.25589736129417 & 0.19337377390523 & 36498 \\
\hline & -1.74527494587302 & -0.90320106882472 & 36800 \\
\hline & -1.43038449671731 & -2.00090103055200 & 74128 \\
\hline & -0.39185690888227 & 7305537611 & \\
\hline & -2.42145472844 & 407021565 & 3073 \\
\hline & -2.14386679199729 & 6644 & 08732 \\
\hline & -3.76 & 4225 & \\
\hline & -4.5 & 0109 & 36682 \\
\hline & -4.05 & & \\
\hline & -5.1 & 9837 & 91692 \\
\hline & -3.10 & & \\
\hline & -3.56 & & 6360 \\
\hline & & & \\
\hline & 0.7225 & -1.08 & \\
\hline & -0.6610 & -3.4 & 40316 \\
\hline & -2.17 & -2.1 & 9027 \\
\hline & 1.286 & -2.31 & -2.2 \\
\hline & 1.29 & -0.1 & -2.1 \\
\hline & 0.59 & -3.4 & \\
\hline & & & \\
\hline & & & \\
\hline & & -4.4 & -2.1 \\
\hline & -0.46 & & 5589 \\
\hline & 1.330 & & 3454 \\
\hline & 3.203 & 2.68 & 6948 \\
\hline & -1.470 & 405633 & 17193 \\
\hline & 2.189 & 2.80 & 8205 \\
\hline & 0.6212 & & \\
\hline & 0.43 & 203 & \\
\hline & 1.14 & 2.46 & 4861 \\
\hline & 1.9299 & 3.34 & \\
\hline & -2.015 & 3539 & 03521 \\
\hline O & 2.912 & & \\
\hline & 4.269151 & 3.43 & 1.02067094233364 \\
\hline & & & \\
\hline$H$ & 4.41840977120035 & 2.36558408317364 & 0.84011530658634 \\
\hline & 4.57694322810054 & 3439797022 & \\
\hline & -3.87879170535573 & & 0.67570631683953 \\
\hline & -3.90499198946873 & 1.48664840980962 & -0.18896394216431 \\
\hline & -4.23102146834000 & -0.87272562478556 & 0.16462234474782 \\
\hline
\end{tabular}




$\begin{array}{lrrr}\mathrm{C} & -4.29336326191266 & 1.34848451433089 & -1.52547468899700 \\ \mathrm{H} & -3.64935576760142 & 2.46965076018919 & 0.19675227073817 \\ \mathrm{C} & -4.58932853986382 & -1.02662776828196 & -1.17696187109165 \\ \mathrm{H} & -4.21478894441722 & -1.74045223246248 & 0.81794035890700 \\ \mathrm{C} & -4.62847992240816 & 0.07909460845358 & -2.02993505545717 \\ \mathrm{H} & -4.35264295493819 & 2.22398716207404 & -2.16877709769402 \\ \mathrm{H} & -4.84470975572174 & -2.01171964739068 & -1.55812432685979 \\ \mathrm{H} & -4.92797843083935 & -0.03457426076541 & -3.06732328764554 \\ \mathrm{H} & -4.46921648578069 & 1.01124423994559 & 2.60993818331093 \\ \mathrm{H} & -2.82592561152743 & 1.41291800903852 & 2.18335599467370\end{array}$

$\mathrm{TS}_{12-8} \mathrm{~F}-\mathrm{oBn}\left(\mathrm{Pd}(0)-\mathrm{Cy} 2 \mathrm{P}\left(o-B n-\mathrm{C}_{6} \mathrm{H}_{4}\right)\right.$ complex with $\mathrm{Pd}$-aryl binding with $\mathrm{PhMgBr}$ bound and 4-methoxyfluorobenzene TS)

$\begin{array}{cccc}\text { Final Gibbs free enthalpy }\left(\Delta \mathrm{G}=\Delta \mathrm{H}-\mathrm{T}^{*} \Delta \mathrm{S}\right)=-4892.901294 E_{\mathrm{h}} \\ \mathrm{C} & -0.41221128323314 & -0.94277197646782 & -1.15120976386935 \\ \mathrm{C} & -0.07627521277882 & 2.43414752177620 & -1.02335514880654 \\ \mathrm{Pd} & -0.60332631982768 & 0.79647515224467 & 0.11113649335840 \\ \mathrm{Mg} & -1.61898520025608 & 0.61056678206949 & -2.33147413564263 \\ \mathrm{~F} & -1.06936160607131 & 2.52533751497532 & -2.38806172536753 \\ \mathrm{P} & -0.38138182535400 & -0.04506213330308 & 2.33277282041897 \\ \mathrm{C} & 1.09259846539008 & -1.17657372776801 & 2.41931938148073 \\ \mathrm{H} & 1.29695607452542 & -1.41309781355673 & 3.47123009583409 \\ \mathrm{C} & 2.31339508885047 & -0.42996577922432 & 1.85092892565681 \\ \mathrm{H} & 2.08766979448091 & -0.14074727059502 & 0.81624263148917 \\ \mathrm{H} & 2.49778951414572 & 0.49837143827632 & 2.40241296007808 \\ \mathrm{C} & 3.56901538887434 & -1.30644513905569 & 1.88870437452723 \\ \mathrm{H} & 4.40745756153610 & -0.76467852562002 & 1.43188437477377 \\ \mathrm{H} & 3.84422096899965 & -1.49202421501154 & 2.93772148722998 \\ \mathrm{C} & 3.35173616938873 & -2.64510400702169 & 1.17848375303761 \\ \mathrm{H} & 4.25053413010443 & -3.26898023573993 & 1.26747871325875 \\ \mathrm{H} & 3.18647088945766 & -2.46172593313754 & 0.10739546041619 \\ \mathrm{C} & 2.13164867542416 & -3.37597421152218 & 1.74413849372910 \\ \mathrm{H} & 1.95713276214996 & -4.30899730913402 & 1.19357803716129 \\ \mathrm{H} & 2.32245254658030 & -3.65120332553761 & 2.79253475291590 \\ \mathrm{C} & 0.88450089740242 & -2.49302863064602 & 1.66113508013524 \\ \mathrm{H} & 0.00874978949837 & -3.02823732089550 & 2.04159926401318 \\ \mathrm{H} & 0.68562681987933 & -2.26529769928334 & 0.61437449555226 \\ \mathrm{C} & 0.08424455242717 & 1.29329576994611 & 3.56448339483276 \\ \mathrm{H} & 0.92079827421661 & 1.80157186447348 & 3.06515697073189 \\ \mathrm{C} & -1.02508846453450 & 2.33517433835944 & 3.74898085153446 \\ \mathrm{H} & -1.33397311959198 & 2.72505864537525 & 2.77500197522958 \\ \mathrm{H} & -1.89970433874152 & 1.85645373324226 & 4.20826965260645 \\ \mathrm{C} & -0.56327561759725 & 3.49024426764169 & 4.64235254804142 \\ \mathrm{H} & 0.26193789756153 & 4.01933374261315 & 4.14345857366002 \\ \mathrm{H} & -1.38094257247587 & 4.21195868610930 & 4.76416749972772\end{array}$




\begin{tabular}{|c|c|c|c|}
\hline & -0.08656645883792 & & \\
\hline & -0.93744030978530 & 2.54075820118481 & \\
\hline & .28079220614407 & 3.81849357207312 & 6.61527178373026 \\
\hline & 1.00686642053380 & & \\
\hline & & & .81387773053042 \\
\hline & & & 5.39688907349326 \\
\hline & 0.55474474292845 & & \\
\hline & .27286773076956 & 0.23531431068182 & 00475 \\
\hline & 1.3788129603 & 0.066 & \\
\hline & -1.7566306053 & -0.93 & 00144 \\
\hline & -1.4891 & & \\
\hline & -0.4631 & -2.31 & 40731 \\
\hline & -2.51 & -2.72 & \\
\hline & -2.27291153 & -3.56 & 8749 \\
\hline & $-3.838902815^{\prime}$ & & \\
\hline & -4.6503 & -2.89 & \\
\hline & -4.11755027 & -1.26 & 880 \\
\hline H & -5.15 & -0.9 & \\
\hline & -3.10 & -0.54 & 772 \\
\hline & -3.50 & & \\
\hline & -1.1868 & -2.11 & 364 \\
\hline & 0.82945 & -1.07 & \\
\hline C & -0.74839402 & & 570 \\
\hline H & -2.13839008 & -2.07 & 789 \\
\hline$C$ & 1.2831 & -2.29 & \\
\hline & 1.4690 & -0.19 & -1.9 \\
\hline $\mathrm{C}$ & 0.49 & -3.44 & \\
\hline & $-1.3^{\prime}$ & -4.22 & 547 \\
\hline H & 2.25 & -2.363 & 315 \\
\hline & 0.84 & -4.397 & \\
\hline $\mathrm{C}$ & & & \\
\hline & 1.61334 & 2.013 & \\
\hline$H$ & 3.22996 & 3.169 & \\
\hline H & -1.60 & & 866 \\
\hline C & 2.18 & & -0.6 \\
\hline $\mathrm{C}$ & 0.38634 & 3.918 & \\
\hline & 0.04658 & & \\
\hline $\mathrm{C}$ & 1.277032050 & 2.508 & -1.41 \\
\hline & 1.7408956100 & 3.880633657 & \\
\hline $\mathrm{Br}$ & & & \\
\hline $\mathrm{O}$ & 2.57677685600240 & 4.5705588654 & 663891 \\
\hline $\mathrm{C}$ & 3.97934028 & 4.53396 & \\
\hline & 4.45036443501150 & 5.11024552701837 & 1.913941 \\
\hline & 4.36109643004918 & & \\
\hline $\mathrm{H}$ & 4.21306052566828 & 4.99312540397872 & 0.14369413155044 \\
\hline & -3.84249385278636 & 0.34618486972339 & 0.66369963150544 \\
\hline
\end{tabular}




$\begin{array}{lrrr}\mathrm{C} & -3.84120898282066 & 1.41271686819362 & -0.25017455508757 \\ \mathrm{C} & -4.22858232891610 & -0.91665614490623 & 0.20942715548516 \\ \mathrm{C} & -4.22139016722189 & 1.22233642994537 & -1.58181198156798 \\ \mathrm{H} & -3.57008841715199 & 2.40718310541299 & 0.09213351614117 \\ \mathrm{C} & -4.59387649083781 & -1.12011087869011 & -1.12368688740902 \\ \mathrm{H} & -4.23681055974870 & -1.75499222779433 & 0.90073636974899 \\ \mathrm{C} & -4.59154572173629 & -0.05702526450997 & -2.02665821704124 \\ \mathrm{H} & -4.25416947282460 & 2.06937596353261 & -2.26489922799866 \\ \mathrm{H} & -4.87901615814182 & -2.11370660271540 & -1.45905872238391 \\ \mathrm{H} & -4.88093109192633 & -0.21188442909381 & -3.06117199066584 \\ \mathrm{H} & -4.38746855661533 & 1.09817504026950 & 2.57257597155570 \\ \mathrm{H} & -2.72951575071388 & 1.39872018418123 & 2.12080916527001\end{array}$

12F-xyl (Pd(0)-DavePhos complex with Pd-aryl binding with PhMgBr bound and 2,6-dimethylfluorobenzene bound)

Final Gibbs free enthalpy $\left(\Delta \mathrm{G}=\Delta \mathrm{H}-\mathrm{T}^{*} \Delta \mathrm{S}\right)=-4951.57618265 E_{\mathrm{h}}$
C -0.32401045645814
$-0.28706358054777$
$-1.35823992535136$
C 0.32632624411973
3.05145629159053
$-0.87406897366727$
Pd -0.32003885374908
1.27991087667334
0.24534429626649
$\mathrm{Mg}-1.70231304954871$
1.45944397570773
$-1.89468766189931$
F -0.82573268801434
3.45846878527913
$-1.68701976789581$
P $\quad-0.39576820506407$
0.29310944799182
2.40696882332036
C 0.95000428878478
$-0.98319858693352$
2.49472970669854
H 1.09616845939960
$-1.28829406032475$
3.53895718191267
C 2.25105930442244
$-0.34202211195884$
1.98638964470500
H 2.06796216272330
0.05140381050089
0.97721902940143
H 2.52452151717908
0.52007882936802
2.60574460803507
C 3.40098665216355
$-1.35148881507473$
1.96026335279563
H 4.29974606937067
$-0.87458694995066$
1.54811028277272
H 3.64229095516071
$-1.64895825983339$
2.99193836293097
C 3.03958364583347
$-2.59615009635932$
1.14658226174801
H 3.86132756221224
$-3.32353360312744$
1.18178627132514
H 2.90331727891113
$-2.31203717142042$
0.09475157713959
C 1.74433190341321
$-3.23422677407310$
1.65525575998457
H 1.46984362492475
$-4.09137745444871$
1.02723376697920
H 1.90602473212118
$-3.62134771915469$
2.67288664838353
C 0.59139841800048
$-2.22618106582650$
1.67357318046144
H -0.30640091546823
$-2.69688916464879$
2.08895514409050
H 0.35787989818661
$-1.92403964093092$
0.64835149318462
C 0.07884787730891
1.50455085381625
3.76117793299508
H 0.84626385334580
2.10844805878066
3.25983778527648
C -1.10123501607889
2.42893895311515
4.08512926589242
H -1.52285633122555
2.82908437425662
3.15974134601402
H -1.89173513381204
1.84266604166344
4.57167447945282
C -0.67951597272225
3.58109499505776
5.00125759084427
H 0.03971008483110
4.21945926420363
4.46617114999708 


\begin{tabular}{|c|c|c|c|}
\hline & -1.54899703969571 & & \\
\hline & -0.02804342240386 & & \\
\hline & 0.78033093718476 & 2.51829394939287 & \\
\hline & .31817160838205 & 3.90154087799522 & \\
\hline & 1.13510016369953 & & \\
\hline & & & 7370 \\
\hline & & & \\
\hline & 0.68968248347352 & 0.964 & \\
\hline & -0.05491525836207 & & 5763 \\
\hline & 1.54343436192885 & 0.311 & \\
\hline & & & 039 \\
\hline & -1.7260 & 3996 & 182 \\
\hline & -0.7404 & & \\
\hline & -2.8135 & -1.93 & 7280 \\
\hline & -2.66524194704916 & 9503 & \\
\hline & -4.09 & -1.77 & \\
\hline & -4.95 & -2.22 & 345 \\
\hline & -4.24 & -1.04 & \\
\hline & -5.23 & -0.9 & 298 \\
\hline & -3.15 & -0.4 & \\
\hline & -3.45 & 0.25 & 077 \\
\hline & -3.33 & & \\
\hline & -2.96 & & \\
\hline & -3.76 & 2.37 & \\
\hline 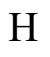 & -3.71 & & \\
\hline & -4.31 & 1.69 & -1.00 \\
\hline 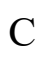 & -4.35 & & \\
\hline & -4.79 & -0.20 & 829 \\
\hline & -3.99 & 9363 & 200 \\
\hline & -1.16 & 8243 & 321 \\
\hline $\mathrm{C}$ & & & \\
\hline & -0.69 & -2.63 & -2.03 \\
\hline $\mathrm{H}$ & -2.21 & -1.34 & \\
\hline & 1.51 & -1.68 & -2.216 \\
\hline & 1.72 & & -1.626 \\
\hline & 0.653 & -2.7776 & -2.366 \\
\hline & -1.379 & -3.466 & \\
\hline & 2.563 & -1.781 & -2.4777 \\
\hline & 1.029 & -3.725 & -2.7448 \\
\hline $\mathrm{C}$ & & & \\
\hline & 1.4367 & 01956946 & -3.115076593 \\
\hline $\mathrm{H}$ & 3.62857571906667 & 2.63870 & -1.47148222355521 \\
\hline $\mathrm{C}$ & -0.77752848842761 & 4.40231092431443 & 1.00401740356649 \\
\hline & 2.7085 & & -0.93599393759587 \\
\hline & 1.60250879561478 & 3.54162891916126 & 1.10908956509035 \\
\hline & 1.65645056718841 & 3.91600923050195 & 2.12888962668841 \\
\hline
\end{tabular}




$\begin{array}{llcc}\mathrm{C} & 1.51246384608608 & 2.84593709117902 & -1.63358240305668 \\ \mathrm{C} & 2.75710621555957 & 3.19589825399325 & 0.43499814648132 \\ \mathrm{Br} & -2.46434839136547 & 1.56848237039340 & -4.23941887957407 \\ \mathrm{H} & -4.67672405953444 & 2.23296219418188 & -1.87895598220086 \\ \mathrm{~N} & -4.16405919508702 & -1.83147827258288 & 0.14303322756391 \\ \mathrm{C} & -3.21116467515274 & -2.75374319134059 & 0.75493104913699 \\ \mathrm{H} & -2.90781156860036 & -3.49921309090955 & 0.01013839265362 \\ \mathrm{H} & -2.31583096657103 & -2.22667577746709 & 1.07419084651941 \\ \mathrm{H} & -3.64354650398566 & -3.27285282860988 & 1.61990646362756 \\ \mathrm{C} & -5.03918062812821 & -2.45266647885453 & -0.83720229186897 \\ \mathrm{H} & -5.31844138672393 & -3.44386023007775 & -0.46822218472117 \\ \mathrm{H} & -5.95225365025820 & -1.86450348169933 & -0.96533692555695 \\ \mathrm{H} & -4.55658374623049 & -2.57740900517535 & -1.82152299062130 \\ \mathrm{H} & 3.71415654771006 & 3.20990371302102 & 0.95024798216983 \\ \mathrm{H} & -1.76874657043788 & 4.01713288019157 & 0.77148402625757 \\ \mathrm{H} & -0.70041920122964 & 5.40724621234662 & 0.56640274927667 \\ \mathrm{H} & -0.68559942290721 & 4.50686086274288 & 2.08771579767839 \\ \mathrm{H} & 0.76047207680093 & 1.80501767423748 & -3.37839245896136 \\ \mathrm{H} & 2.42571828488403 & 2.39555071383460 & -3.51663581902377 \\ \mathrm{H} & 1.05500884321922 & 3.52395472648869 & -3.62406319446266\end{array}$

TS $_{12-8}$ F-xyl (Pd(0)-DavePhos complex with Pd-aryl binding with PhMgBr bound and 2,6-dimethylfluorobenzene bound TS)

Final Gibbs free enthalpy $\left(\Delta \mathrm{G}=\Delta \mathrm{H}-\mathrm{T}^{*} \Delta \mathrm{S}\right)=-4951.57210085 E_{\mathrm{h}}$

$\begin{array}{llcc}\mathrm{C} & -0.27426337488452 & -0.20414909426749 & -1.19199535252042 \\ \mathrm{C} & 0.37784627983883 & 3.01710769548971 & -0.70883654519189 \\ \mathrm{Pd} & -0.36060412264283 & 1.37525555088762 & 0.27464947296386 \\ \mathrm{Mg} & -1.59374413440293 & 1.69364655109279 & -1.97811197983581 \\ \mathrm{~F} & -0.93341803169786 & 3.53366701247303 & -1.88999540291044 \\ \mathrm{P} & -0.35787245790518 & 0.22969294322491 & 2.36382399934395 \\ \mathrm{C} & 0.94281857954986 & -1.08652393482521 & 2.45231493208182 \\ \mathrm{H} & 1.07477550874828 & -1.37106735486708 & 3.50402886598257 \\ \mathrm{C} & 2.27340369090423 & -0.51277800780542 & 1.93966041107146 \\ \mathrm{H} & 2.13057646545946 & -0.16256893910072 & 0.91112040179762 \\ \mathrm{H} & 2.57040830794442 & 0.36175285907778 & 2.52939822092321 \\ \mathrm{C} & 3.38018562865295 & -1.57091981646381 & 1.97452806347931 \\ \mathrm{H} & 4.30721350935414 & -1.14585333135427 & 1.56846827974293 \\ \mathrm{H} & 3.58524281174584 & -1.84452121177929 & 3.02051923042049 \\ \mathrm{C} & 2.98064153870931 & -2.82445263247173 & 1.19132809720464 \\ \mathrm{H} & 3.76923589711850 & -3.58435816328987 & 1.26475694930709 \\ \mathrm{H} & 2.87906701945103 & -2.56581631661965 & 0.12867017161229 \\ \mathrm{C} & 1.65056820917212 & -3.39199869976891 & 1.69339358515704 \\ \mathrm{H} & 1.35181683624830 & -4.25625246333921 & 1.08635315434543 \\ \mathrm{H} & 1.77797751338059 & -3.75308584178282 & 2.72529494222527 \\ \mathrm{C} & 0.53852908975912 & -2.33856980247469 & 1.66457263819943\end{array}$




\begin{tabular}{|c|c|c|c|}
\hline & -0.37919606050269 & -2.76084761803622 & \\
\hline & 0.32188869556941 & -2.06235784308170 & \\
\hline & 16881397846136 & & \\
\hline & 91028800403769 & & \\
\hline & & & 4.02462197263605 \\
\hline & -1.46931510948313 & & 92013 \\
\hline & -1.77693435738444 & & \\
\hline & .567819594531 & & 4.9 \\
\hline & & & \\
\hline & -1.43581781085504 & 135682 & 6095 \\
\hline & & & \\
\hline & -0.59 & 168 & 5601 \\
\hline & & & \\
\hline & 1.293 & 2.12013 & 5.83 \\
\hline & & & 9257 \\
\hline & 2.06 & 2.68 & \\
\hline & 0.83 & 0.949 & 4.9 \\
\hline & & & \\
\hline & 1.68 & 0.30 & 4.72 \\
\hline & & -0.5 & \\
\hline & -1.71 & $-1.2 \varepsilon$ & 2694 \\
\hline & -0.73 & & \\
\hline & -2.80 & & \\
\hline & -2.66 & -2.42 & \\
\hline & -4.08 & -1.72 & \\
\hline & -4.94 & -2.15 & 199 \\
\hline 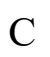 & -4.23 & & \\
\hline & -5.2 & -0.9 & 3957 \\
\hline & -3.14 & -0.45 & 9611 \\
\hline & -3.44 & & \\
\hline$y$ & & & \\
\hline & -2.92 & & 8339 \\
\hline & -3.76 & 2.296 & 9023 \\
\hline & -3.71 & & 6789 \\
\hline & -4.36 & & \\
\hline & -4.48 & 0.193 & 7329 \\
\hline & -4.90 & -0.340 & \\
\hline $\mathrm{C}$ & -4.04 & -0.525 & 47342 \\
\hline & -1.19 & 4931 & 72578 \\
\hline $\mathrm{C}$ & & -0.4399671 & -1.565 \\
\hline & $-0.80^{\top}$ & -2.412 & 91726 \\
\hline $\mathrm{H}$ & -2.2401239218 & -1.0994685 & -1.24266760922102 \\
\hline & 1.46453303798832 & -1.62719880120335 & -2.17683947639429 \\
\hline & & & -1.35740703493995 \\
\hline & 0.52753036550907 & -2.62608905048838 & -2.45629797303032 \\
\hline & -1.55466834589474 & -3.17274633840513 & -2.34279517547662 \\
\hline
\end{tabular}




$\begin{array}{lllc}\text { H } & 2.51171157307824 & -1.77684166399023 & -2.43392910142029 \\ \mathrm{H} & 0.83587460243430 & -3.55293339347747 & -2.93520558755152 \\ \mathrm{C} & 0.24582702633850 & 3.71186278259129 & 0.52977637309999 \\ \mathrm{C} & 1.63170963942664 & 2.53681463452875 & -2.87303258661157 \\ \mathrm{H} & 3.71177094086408 & 3.04762296633846 & -1.17799979159816 \\ \mathrm{C} & -0.99902566704780 & 4.46210637458250 & 0.93117402334019 \\ \mathrm{C} & 2.74688501545246 & 3.21371013988808 & -0.70176275234686 \\ \mathrm{C} & 1.46750403888798 & 3.98984070795502 & 1.19864339492063 \\ \mathrm{H} & 1.42273479144409 & 4.50951120967338 & 2.15421616032329 \\ \mathrm{C} & 1.59571473373130 & 2.92742923245789 & -1.42502363586380 \\ \mathrm{C} & 2.69032649075208 & 3.71117119568003 & 0.61593853296281 \\ \mathrm{Br} & -2.40017225190195 & 1.24855644002250 & -4.26693752100556 \\ \mathrm{H} & -4.73500652482485 & 2.09496477031624 & -1.96467945607726 \\ \mathrm{~N} & -4.20990599854458 & -1.89934169813340 & 0.18743800561374 \\ \mathrm{C} & -3.19809165548930 & -2.79256037193223 & 0.74400924396733 \\ \mathrm{H} & -2.90486765428503 & -3.52587101973578 & -0.01801630464834 \\ \mathrm{H} & -2.30698477061394 & -2.23605549661083 & 1.02131206546278 \\ \mathrm{H} & -3.57005102138291 & -3.32972870341497 & 1.62603290787404 \\ \mathrm{C} & -5.12391973403099 & -2.55033467835426 & -0.73559874289399 \\ \mathrm{H} & -5.38235835603926 & -3.53252768889981 & -0.32706182213722 \\ \mathrm{H} & -6.04433420423587 & -1.96810873541400 & -0.83806321422999 \\ \mathrm{H} & -4.68507462577124 & -2.70148222580284 & -1.73688756247320 \\ \mathrm{H} & 3.61274148255414 & 3.92254960760278 & 1.15073221499022 \\ \mathrm{H} & -1.90812538371135 & 3.97904920649999 & 0.58481539927154 \\ \mathrm{H} & -0.97362219372793 & 5.46805124460774 & 0.49062191322740 \\ \mathrm{H} & -1.04583100476441 & 4.57849358251213 & 2.01698475203425 \\ \mathrm{H} & 0.96068539361303 & 1.69869851318434 & -3.09489285582955 \\ \mathrm{H} & 2.64363172432995 & 2.24654197844683 & -3.17033395056505 \\ \mathrm{H} & 1.31109358649390 & 3.37744862086929 & -3.50002497492013\end{array}$

\section{2,6-dimethylfluorobenzene}

Final Gibbs free enthalpy $\left(\Delta \mathrm{G}=\Delta \mathrm{H}-\mathrm{T}^{*} \Delta \mathrm{S}\right)=-409.90716197 E_{\mathrm{h}}$
C 0.36518545940576
3.19337831361556
$-0.85639760182020$
F -0.84504343309098
3.20126449041638
$-1.49892667386203$
C 0.37496204228844
3.47708149457445
0.51001815613162
C 1.39554604612958
2.61406646171760
$-3.08873207932311$
H 3.63274296751686
2.68120005810947
$-1.49308780524863$
C -0.89773828499644
3.78254524484994
1.25067874986538
C 2.72444286857504
2.90309543231034
$-0.93678524576433$
C 1.62514182179786
3.46418602841047
1.14215624817780
H 1.67687539435481
3.68506517815921
2.20640722193831
C 1.49937532978647
2.90451510652141
$-1.61658257528081$
C 2.78975048339094
3.17947060835123
0.42883343003124
H 3.75033931470340
3.17509012478039
0.93854261846789
H -1.60154307821897
2.94270200556147
1.19240585396294 


$\begin{array}{llll}\mathrm{H} & -1.40399150918522 & 4.65633336091177 & 0.82251410329100 \\ \mathrm{H} & -0.68637952931865 & 3.98656929425913 & 2.30400479035405 \\ \mathrm{H} & 0.76317384023226 & 1.73711695835632 & -3.27519068494084 \\ \mathrm{H} & 2.38595443320180 & 2.42355721905610 & -3.51045659915210 \\ \mathrm{H} & 0.94354583342704 & 3.45587262003877 & -3.62769190682818\end{array}$

cis-MgBrCl(dme $)_{2}$

Final Gibbs free enthalpy $\left(\Delta \mathrm{G}=\Delta \mathrm{H}-\mathrm{T}^{*} \Delta \mathrm{S}\right)=-3853.39615296 E_{\mathrm{h}}$

$\begin{array}{llll}\mathrm{Mg} & -7.69942519448120 & -3.08337528367735 & 2.05390771652087 \\ \mathrm{Br} & -8.26625304888842 & -2.45157755321688 & 4.54962144020290 \\ \mathrm{C} & -5.81501646304328 & -5.08647872261675 & 3.55122865298760 \\ \mathrm{H} & -6.75202245130744 & -5.63862592032581 & 3.45536000420660 \\ \mathrm{H} & -5.76115441169097 & -4.61365817082036 & 4.53858204598501 \\ \mathrm{H} & -4.95916374314133 & -5.75343825923944 & 3.39304252638952 \\ \mathrm{C} & -4.67300339513602 & -3.22329878212701 & 2.49323970665962 \\ \mathrm{H} & -4.30223503684847 & -3.20763392971223 & 1.46340776652283 \\ \mathrm{H} & -3.89064178170755 & -3.62685610101726 & 3.14369513143162 \\ \mathrm{C} & -5.05523627432231 & -1.83240018776138 & 2.93782883907199 \\ \mathrm{H} & -5.36132808005570 & -1.81933372917188 & 3.99252689235276 \\ \mathrm{H} & -4.20837511489871 & -1.14798779447763 & 2.78890382344792 \\ \mathrm{C} & -6.55300212731414 & -0.05998611278651 & 2.39699405742663 \\ \mathrm{H} & -7.40122835821089 & 0.16561718974605 & 1.74950598540934 \\ \mathrm{H} & -5.71473294153969 & 0.61299926353918 & 2.17438784232620 \\ \mathrm{H} & -6.85586551708845 & 0.03935196685691 & 3.44655092665460 \\ \mathrm{C} & -6.02776737849125 & -3.68090317812580 & -0.72926601094930 \\ \mathrm{H} & -5.70463426399836 & -4.51758550303321 & -0.10754110857889 \\ \mathrm{H} & -5.22231927682719 & -2.93863555315074 & -0.81820529461384 \\ \mathrm{H} & -6.30268148764577 & -4.04386272022421 & -1.72704659942367 \\ \mathrm{C} & -7.74613632854400 & -2.00697142615212 & -0.80403924301678 \\ \mathrm{H} & -7.77949974535002 & -2.22976131520655 & -1.87833294371319 \\ \mathrm{H} & -7.13633580974495 & -1.10678460965389 & -0.64128225335326 \\ \mathrm{C} & -9.15317954340018 & -1.82726786068908 & -0.29319188186722 \\ \mathrm{H} & -9.59963817120390 & -0.92467542117622 & -0.73037882094502 \\ \mathrm{H} & -9.76961562478306 & -2.70172349622304 & -0.54325596151363 \\ \mathrm{C} & -10.39916210257915 & -1.48837039791467 & 1.71506775715110 \\ \mathrm{H} & -10.24987280984043 & -1.38677216237184 & 2.79118268429330 \\ \mathrm{H} & -11.03915901186421 & -2.35358465588139 & 1.50236002542623 \\ \mathrm{H} & -10.83971909983477 & -0.57573806776303 & 1.29582880890413 \\ \mathrm{Cl} & -9.02102864210510 & -5.05490514042296 & 1.77755934614704 \\ \mathrm{O} & -5.83410034263298 & -4.08349740770850 & 2.51348883167075 \\ \mathrm{O} & -6.16543188675277 & -1.41735712680224 & 2.12089720688606 \\ \mathrm{O} & -7.17431853465291 & -3.11096762708198 & -0.08959754724522 \\ \mathrm{O} & -9.09260600007440 & -1.69182420361018 & 1.13841964714542\end{array}$


cis-MgBrF(dme)

Final Gibbs free enthalpy $\left(\Delta \mathrm{G}=\Delta \mathrm{H}-\mathrm{T}^{*} \Delta \mathrm{S}\right)=-3492.98327806 E_{\mathrm{h}}$

$\begin{array}{llll}\mathrm{Mg} & -7.70482980592342 & -3.09356785605386 & 2.06708331593789 \\ \mathrm{Br}-8.22530550302697 & -2.36013661169159 & 4.59097358372279 \\ \mathrm{C} & -5.88290862172274 & -5.18640625701761 & 3.47944752278108 \\ \mathrm{H} & -6.83293534239101 & -5.69753801248978 & 3.31134958067614 \\ \mathrm{H} & -5.83741919753105 & -4.81371604763146 & 4.51024034106390 \\ \mathrm{H} & -5.04268806813274 & -5.86140720338262 & 3.27581971741016 \\ \mathrm{C} & -4.66634725572042 & -3.26963490943904 & 2.60501333933910 \\ \mathrm{H} & -4.18019486317378 & -3.32507226858731 & 1.62464189399590 \\ \mathrm{H} & -3.97536304479078 & -3.65128450877755 & 3.36381773134579 \\ \mathrm{C} & -5.05053624876630 & -1.84732980080439 & 2.94103523213071 \\ \mathrm{H} & -5.39575937646423 & -1.76704258680344 & 3.98023073657066 \\ \mathrm{H} & -4.19084962449292 & -1.18154836999237 & 2.78233255391160 \\ \mathrm{C} & -6.49527383067675 & -0.08892163975947 & 2.24673336381060 \\ \mathrm{H} & -7.32959172089205 & 0.10917254098385 & 1.57300190624936 \\ \mathrm{H} & -5.64209082090903 & 0.55535367426316 & 1.99732118612923 \\ \mathrm{H} & -6.81226196180185 & 0.08200215118890 & 3.28301898476543 \\ \mathrm{C} & -5.93669112357679 & -3.49420140484627 & -0.68227139008149 \\ \mathrm{H} & -5.56476758202527 & -4.34233225116535 & -0.10502421239181 \\ \mathrm{H} & -5.23790578138264 & -2.64895636934959 & -0.60798546565601 \\ \mathrm{H} & -6.05361762030772 & -3.78404295407280 & -1.73439887353267 \\ \mathrm{C} & -7.84376331269494 & -2.07177490104726 & -0.83652476987096 \\ \mathrm{H} & -7.90961362212164 & -2.31268224709831 & -1.90633877530622 \\ \mathrm{H} & -7.25669960605196 & -1.14942247317726 & -0.71401042985514 \\ \mathrm{C} & -9.23250729320692 & -1.91324712992842 & -0.27447607652221 \\ \mathrm{H} & -9.72494478328386 & -1.05059936495573 & -0.74270146532876 \\ \mathrm{H} & -9.83198529664896 & -2.81667745498434 & -0.45399327324833 \\ \mathrm{C} & -10.40037916043853 & -1.46828645943034 & 1.75903164906310 \\ \mathrm{H} & -10.21064733772745 & -1.32034932047887 & 2.82345641602377 \\ \mathrm{H} & -11.04905061116797 & -2.34070508963771 & 1.60930049691158 \\ \mathrm{H} & -10.86093566777648 & -0.57513999824066 & 1.31872405707271 \\ \mathrm{~F} & -8.73501443271674 & -4.63814128113378 & 1.92634465650772 \\ \mathrm{O} & -5.85421237695104 & -4.08545043919602 & 2.55043952908755 \\ \mathrm{O} & -6.12760397418216 & -1.46728262053573 & 2.06393345468787 \\ \mathrm{O} & -7.20973214431474 & -3.14500631837462 & -0.12924822213795 \\ \mathrm{O} & -9.11790298700819 & -1.69605421635233 & 1.14265170473692\end{array}$

cis- $\operatorname{MgBr}_{2}(\mathrm{dme})_{2}$

Final Gibbs free enthalpy $\left(\Delta \mathrm{G}=\Delta \mathrm{H}-\mathrm{T}^{*} \Delta \mathrm{S}\right)=-5968.63442540 E_{\mathrm{h}}$

$\begin{array}{lll}\mathrm{Mg}-7.69187021163456 & -3.07216132245395 & 2.05586393844440 \\ \mathrm{Br}-8.29461141736292 & -2.50635003879573 & 4.53011851158405 \\ \mathrm{C}-5.79013915707066 & -5.07963808284003 & 3.54756668707183\end{array}$




$\begin{array}{llll}\mathrm{H} & -6.72297034433731 & -5.64074417934034 & 3.46363721631176 \\ \mathrm{H} & -5.72589341623303 & -4.61004347804791 & 4.53573895237281 \\ \mathrm{H} & -4.93100308694294 & -5.73817131289203 & 3.37394408235235 \\ \mathrm{C} & -4.67643111291335 & -3.20560475773526 & 2.47879054978924 \\ \mathrm{H} & -4.32376542951977 & -3.18059183305490 & 1.44285690107659 \\ \mathrm{H} & -3.88094066280761 & -3.60968013863103 & 3.11257740273616 \\ \mathrm{C} & -5.05859103497525 & -1.82056523493750 & 2.94119589792536 \\ \mathrm{H} & -5.35332148061255 & -1.81976458332396 & 3.99930735339473 \\ \mathrm{H} & -4.21633291384622 & -1.13115936895545 & 2.79028784325309 \\ \mathrm{C} & -6.57336484302219 & -0.05039901349424 & 2.43698774943161 \\ \mathrm{H} & -7.43042472743402 & 0.17688993001877 & 1.80180568214276 \\ \mathrm{H} & -5.74243538807581 & 0.63096476948378 & 2.21283104188162 \\ \mathrm{H} & -6.86469124538267 & 0.03504277825060 & 3.49104431774143 \\ \mathrm{C} & -6.05763075345126 & -3.73587110531368 & -0.72721305727883 \\ \mathrm{H} & -5.74532891672410 & -4.56591064857323 & -0.09136487196804 \\ \mathrm{H} & -5.23169759343748 & -3.02353250927031 & -0.85808120093031 \\ \mathrm{H} & -6.37303701436711 & -4.11619903741468 & -1.70610626797005 \\ \mathrm{C} & -7.72859717151115 & -2.00782977641418 & -0.79936289694149 \\ \mathrm{H} & -7.75640986381022 & -2.23885451067721 & -1.87173155003867 \\ \mathrm{H} & -7.11524150070070 & -1.10996736184205 & -0.63899379691358 \\ \mathrm{C} & -9.13873857800143 & -1.81916558396148 & -0.29962755419493 \\ \mathrm{H} & -9.57284716733160 & -0.90930772974808 & -0.73386512019728 \\ \mathrm{H} & -9.76090873579515 & -2.68614623965343 & -0.56044475160347 \\ \mathrm{C} & -10.39711481294529 & -1.47541307180602 & 1.70160412460800 \\ \mathrm{H} & -10.25514239486416 & -1.38074410075617 & 2.77925345077895 \\ \mathrm{H} & -11.04738099595241 & -2.33096799572730 & 1.48057136064332 \\ \mathrm{H} & -10.82158799411283 & -0.55512406637967 & 1.28305569658061 \\ \mathrm{Br} & -9.11502982465615 & -5.21332017909090 & 1.70575348842156 \\ \mathrm{O} & -5.83259952126385 & -4.07252347096742 & 2.51409664085875 \\ \mathrm{O} & -6.17930864684389 & -1.40168691712496 & 2.14031925342068 \\ \mathrm{O} & -7.16588752576222 & -3.10925995948061 & -0.07284101198362 \\ \mathrm{O} & -9.08886451629809 & -1.69432986904944 & 1.13380393719859\end{array}$

\section{cis-MgClF(dme $)_{2}$}

Final Gibbs free enthalpy $\left(\Delta \mathrm{G}=\Delta \mathrm{H}-\mathrm{T}^{*} \Delta \mathrm{S}\right)=-1377.74314766 E_{\mathrm{h}}$

$\begin{array}{llll}\mathrm{Mg} & -7.68742713956356 & -3.10052551810833 & 2.02193630484133 \\ \mathrm{~F} & -8.04077932773767 & -2.57648109808547 & 3.78940062145136 \\ \mathrm{C} & -5.88412861284077 & -4.98551460301524 & 3.63694909143097 \\ \mathrm{H} & -6.79394427130490 & -5.57734800349464 & 3.51613475299205 \\ \mathrm{H} & -5.95902429200472 & -4.37672157814699 & 4.54547320667135 \\ \mathrm{H} & -5.00473031855583 & -5.63958216190873 & 3.68484890633452 \\ \mathrm{C} & -4.64748090664967 & -3.26714049939646 & 2.47491512929640 \\ \mathrm{H} & -4.27026117872150 & -3.23315468612293 & 1.44824232295761 \\ \mathrm{H} & -3.86444733978310 & -3.67317720331960 & 3.12467960313822 \\ \mathrm{C} & -5.04230719570001 & -1.88299014948954 & 2.93032037227798\end{array}$




$\begin{array}{ll}\mathrm{H} & -5.43579144328892 \\ \mathrm{H} & -4.17122929181160 \\ \mathrm{C} & -6.53675881938919 \\ \mathrm{H} & -7.28947278240100 \\ \mathrm{H} & -5.70243545086495 \\ \mathrm{H} & -6.99211701140009 \\ \mathrm{C} & -6.00764204081459 \\ \mathrm{H} & -5.66084114146309 \\ \mathrm{H} & -5.23697000958523 \\ \mathrm{H} & -6.23442221264456 \\ \mathrm{C} & -7.80585129225917 \\ \mathrm{H} & -7.87113943639257 \\ \mathrm{H} & -7.20129353225909 \\ \mathrm{C} & -9.19585247059357 \\ \mathrm{H} & -9.66453154798848 \\ \mathrm{H} & -9.81605792513441 \\ \mathrm{C} & -10.33195189323870 \\ \mathrm{H} & -10.11042895092704 \\ \mathrm{H} & -10.98950294214047 \\ \mathrm{H} & -10.79786740795149 \\ \mathrm{Cl} & -9.01799662293162 \\ \mathrm{O} & -5.79250001535135 \\ \mathrm{O} & -6.06709848881331 \\ \mathrm{O} & -7.19734759993594 \\ \mathrm{O} & -9.06797908755784\end{array}$

$-1.89850885376699$

$-1.21274400083649$

$-0.11326407902516$

0.17152097122255

0.60102102093202

$-0.14210828931338$

$-3.60690555611199$

$-4.44949144717365$

$-2.82375312543228$

$-3.94638151182840$

$-2.02851895648417$

$-2.26440910109389$

$-1.11917536552269$

$-1.85132542032772$

$-0.95841540753795$

$-2.73338861417376$

$-1.50057491611260$

$-1.42588176550842$

$-2.35853996637299$

$-0.57738914901430$

$-5.09038239342977$

$-4.14645328915654$

$-1.42008796557890$

$-3.11712150094033$

$-1.69490581632425$
3.95573599683014

2.88270893022366

2.40134043725828

1.66425253377449

2.38328887520906

3.39889514842288

$-0.77886491283291$

$-0.17814831967483$

$-0.81641639874250$

$-1.79722759956652$

$-0.86103314359080$

$-1.93150635695216$

$-0.72860052098689$

$-0.30175508061405$

$-0.73678337849883$

$-0.51397291339509$

1.77997031866623

2.84581545806134

1.59120123829725

1.41234367678545

1.70940106373851

2.46597090178445

2.03593631139980

$-0.15386062672429$

1.11970804973555

\section{$\mathrm{MgF}_{2}(\mathrm{dme})$}

Final Gibbs free enthalpy $\left(\Delta \mathrm{G}=\Delta \mathrm{H}-\mathrm{T}^{*} \Delta \mathrm{S}\right)=-708.64671578 E_{\mathrm{h}}$

$\begin{array}{llcc}\mathrm{Mg} & -0.47513538023189 & -0.37304263387557 & 0.40524802078846 \\ \mathrm{~F} & 1.29629713264769 & -0.47625691698439 & 0.86982282923384 \\ \mathrm{~F} & -1.98773884349662 & -1.40774701347971 & 0.45868909453292 \\ \mathrm{O} & -0.56330147344710 & 0.70034269776108 & -1.43606885909272 \\ \mathrm{C} & -1.43784578215477 & 1.83204036883270 & -1.21327030648611 \\ \mathrm{H} & -1.39307374863703 & 2.51790068375814 & -2.06624155199028 \\ \mathrm{H} & -2.44625658569074 & 1.41338455202156 & -1.13747593697075 \\ \mathrm{C} & -1.06446663200000 & 2.54657835450726 & 0.06824200570840 \\ \mathrm{H} & -0.06615411379771 & 3.00194634758532 & 0.00671874418462 \\ \mathrm{H} & -1.80053333131659 & 3.32983207147527 & 0.29151133930635 \\ \mathrm{O} & -1.07128067410921 & 1.55617218948405 & 1.11441819019271 \\ \mathrm{C} & -0.62837257614132 & 2.06796533173067 & 2.38511636497213 \\ \mathrm{H} & -1.28624146613836 & 2.88510730954241 & 2.70426177518851 \\ \mathrm{H} & -0.68946224260772 & 1.24070185825901 & 3.09538454740577 \\ \mathrm{H} & 0.40709598183311 & 2.42315837538404 & 2.31218981915910 \\ \mathrm{C} & 0.67156682975451 & 1.03026887479868 & -2.10992143220791 \\ \mathrm{H} & 1.26505269940116 & 1.74253214269003 & -1.52520120479725\end{array}$



H 1.22503475971838
0.09527552024401
$-2.21442828485788$
H $\quad 0.44315544641421$
1.44786988626544
$-3.09697515426991$

10'-MgBr 2 (Pd(0)-DavePhos complex with Pd-aryl binding with $\mathrm{MgBr}_{2}$ bound)

Final Gibbs free enthalpy $\left(\Delta \mathrm{G}=\Delta \mathrm{H}-\mathrm{T}^{*} \Delta \mathrm{S}\right)=-6885.76154996 E_{\mathrm{h}}$

\begin{tabular}{|c|c|c|c|}
\hline & 1.88689750213907 & & \\
\hline & & & \\
\hline & 0.01831806048979 & 17.83251986571682 & \\
\hline & & & \\
\hline & .50451033828358 & 515397 & \\
\hline & & & \\
\hline & 2.42562035430879 & 830 & \\
\hline & 2.16802445769482 & 1601603 & \\
\hline & 3.416675 & & \\
\hline & 2.577 & & \\
\hline & & & \\
\hline & 4.53 & & \\
\hline$\pi$ & & & \\
\hline & 4.12 & & \\
\hline & & & \\
\hline & 6.450 & & \\
\hline & & & \\
\hline C & & & \\
\hline & 6.001 & & \\
\hline $\mathrm{H}$ & & & \\
\hline & 4.85 & & \\
\hline $\mathrm{H}$ & & & \\
\hline & 5.3 & & \\
\hline $\mathrm{H}$ & 5.6 & & \\
\hline & & & \\
\hline $\mathrm{C}$ & & & \\
\hline $\mathrm{H}$ & & & \\
\hline $\mathrm{H}$ & 6.80 & & \\
\hline $\mathrm{C}$ & & & \\
\hline $\mathrm{H}$ & 5.29 & & \\
\hline $\mathrm{H}$ & 6.96 & 204 & \\
\hline 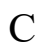 & 5.64 & & \\
\hline $\mathrm{H}$ & 5.31 & & \\
\hline $\mathrm{H}$ & 30268665 & 14.268212 & \\
\hline $\mathrm{C}$ & 4.49 & & \\
\hline $\mathrm{H}$ & 3.62 & & \\
\hline $\mathrm{H}$ & 4.20276931081796 & & \\
\hline $\mathrm{C}$ & 2.97986918898296 & 17.31851890215057 & 26.5831734575295 \\
\hline & & & \\
\hline & 4.98190488396358 & 18.11409334510942 & 26.4387929094711 \\
\hline
\end{tabular}




$\begin{array}{llll}\mathrm{C} & 3.80662257427450 & 18.75384852573126 & 24.78072000809264 \\ \mathrm{H} & 4.62078417248859 & 19.33007889503331 & 24.34786659772740 \\ \mathrm{C} & 2.57758643593926 & 18.66052034174420 & 24.12866828361962 \\ \mathrm{H} & 2.41496264537461 & 19.16484867485395 & 23.17959818849933 \\ \mathrm{C} & 1.56125586792264 & 17.89900106424928 & 24.69609116747899 \\ \mathrm{H} & 0.60257489666761 & 17.81132774010754 & 24.19014601732101 \\ \mathrm{C} & 1.73421384839605 & 17.23238228149217 & 25.92123397276725 \\ \mathrm{C} & 0.59921164427378 & 16.38595573239732 & 26.39109539239296 \\ \mathrm{C} & 0.34058666502428 & 15.22079704406988 & 25.66492055470556 \\ \mathrm{H} & 0.96569054749715 & 14.99848629242042 & 24.80348498786827 \\ \mathrm{C} & -0.65974428127126 & 14.30828998528115 & 26.04643417254108 \\ \mathrm{H} & -0.85945394836496 & 13.42774731081843 & 25.44111508336395 \\ \mathrm{C} & -1.44628247520203 & 14.61039079846720 & 27.16909011434855 \\ \mathrm{H} & -2.24414071351175 & 13.93760014834224 & 27.47435787883346 \\ \mathrm{C} & -1.21780871039445 & 15.77717552500363 & 27.89053554396380 \\ \mathrm{H} & -1.84554539822082 & 15.98486030699367 & 28.74764873874690 \\ \mathrm{C} & -0.20501037438173 & 16.69402386908281 & 27.52988918544248 \\ \mathrm{C} & 0.05366603428193 & 19.14827599933342 & 27.66423215479132 \\ \mathrm{H} & 0.48450466648056 & 19.09487532239045 & 26.66671765582544 \\ \mathrm{H} & 0.66561408898259 & 19.82638947287218 & 28.26905419033690 \\ \mathrm{H} & -0.96117371230201 & 19.57232577760940 & 27.58456299614944 \\ \mathrm{C} & -0.54604461659149 & 17.87246581722913 & 29.63711861325917 \\ \mathrm{H} & -0.33491565378256 & 16.93147076568764 & 30.15315055855312 \\ \mathrm{H} & -1.63410393234093 & 18.05633815436537 & 29.64330549281629 \\ \mathrm{H} & -0.06363592893088 & 18.68604537467857 & 30.18642302635735 \\ \mathrm{Mg} & 0.99864598854018 & 13.19846807840143 & 27.69160691635686 \\ \mathrm{Br} & 2.00566572581513 & 11.42732596570942 & 26.34056767020364 \\ \mathrm{Br} & 0.41766599269480 & 13.27998751058625 & 30.23640713772938\end{array}$

\section{0 '-Mg( $\mu$-Br)F (Pd(0)-DavePhos complex with Pd-aryl binding with MgBrF bound and a bridging bromide)}

Final Gibbs free enthalpy $\left(\Delta \mathrm{G}=\Delta \mathrm{H}-\mathrm{T}^{*} \Delta \mathrm{S}\right)=-4410.11388623 E_{\mathrm{h}}$

$\begin{array}{lccc}\text { Pd } & 1.85476010721725 & 15.08177994808988 & 29.14021564728906 \\ \text { P } & 3.37956389549469 & 16.30732705816081 & 28.07765606349441 \\ \text { N } & 0.02720002523997 & 17.82954595627857 & 28.30185674624573 \\ \text { C } & 4.07568183364933 & 17.43009436768073 & 29.39650379163524 \\ \text { H } & 4.50651871723810 & 16.69162955288359 & 30.09010610227464 \\ \text { C } & 2.93105086558224 & 18.13232322703134 & 30.14055794801163 \\ \text { H } & 2.43608173944082 & 18.82937053376595 & 29.45242427742350 \\ \text { H } & 2.17960275932500 & 17.38618724579901 & 30.42865270745633 \\ \text { C } & 3.43567126586596 & 18.88485571060333 & 31.37319850886698 \\ \text { H } & 2.60087982008653 & 19.41524002099456 & 31.84922920992527 \\ \text { H } & 3.80880018313884 & 18.15282281981931 & 32.10489191434007 \\ \text { C } & 4.55915491044967 & 19.86247604170848 & 31.01780037172456\end{array}$




\begin{tabular}{|c|c|c|c|}
\hline & & & \\
\hline & & & \\
\hline & 69546006778176 & & \\
\hline & & & \\
\hline & & & \\
\hline & & & \\
\hline & 01053621739037 & & \\
\hline & 77788553556075 & & \\
\hline & & & \\
\hline & 642524091 & & 27.220 \\
\hline & & & \\
\hline & 71 & & \\
\hline & & & \\
\hline & 28 & & \\
\hline & & & \\
\hline & 93 & & \\
\hline & 6.10 & & 26. \\
\hline & & & \\
\hline & 79 & & 55 \\
\hline & & & \\
\hline & & & 394 \\
\hline & & & \\
\hline & & & 784 \\
\hline & 3.608 & & \\
\hline & & & \\
\hline & 2.98 & & 57 \\
\hline & & & 37 \\
\hline & 4.98 & & 18 \\
\hline & & & \\
\hline & & & 397 \\
\hline & & & \\
\hline & & & \\
\hline & & & \\
\hline & & & 56 \\
\hline & & & \\
\hline & 0.59 & & \\
\hline & & & \\
\hline & 0.95 & & 24.7 \\
\hline & -0.66 & & 0970 \\
\hline & -0.853070908 & & 25.42051 \\
\hline & -1.43242953037749 & 32928 & 27.16034497703447 \\
\hline & -2.21997001663 & & 27.47041 \\
\hline & -1.20524059203942 & 15.76214556532471 & 27.8923501750129 \\
\hline & & & \\
\hline & -0.20001466497834 & & 27.53328860099423 \\
\hline & -0.01848217894508 & 19.13757659051188 & 27.6532607952716 \\
\hline
\end{tabular}




$\begin{array}{llcc}\mathrm{H} & 0.43952529225872 & 19.10257615906254 & 26.66640130964098 \\ \mathrm{H} & 0.53291718152145 & 19.86179448599515 & 28.26246131538597 \\ \mathrm{H} & -1.05746914125999 & 19.49317954707084 & 27.54506559580188 \\ \mathrm{C} & -0.53030691579669 & 17.85921732463634 & 29.64479668922175 \\ \mathrm{H} & -0.29501839239289 & 16.92380129345515 & 30.16090093978469 \\ \mathrm{H} & -1.62310649139405 & 18.01668243254499 & 29.65900136541697 \\ \mathrm{H} & -0.06423226257943 & 18.68463120421223 & 30.19080763346851 \\ \mathrm{Mg} & 1.16751324248349 & 13.14845582027127 & 27.70155010769815 \\ \mathrm{~F} & 1.79951319241493 & 11.96258540305124 & 26.47767985427409 \\ \mathrm{Br} & 0.37629809856952 & 13.29466813369797 & 30.20500348661269\end{array}$

10 '-MgBr( $\mu$-F) (Pd(0)-DavePhos complex with Pd-aryl binding with MgBrF bound and a bridging fluoride)

Final Gibbs free enthalpy $\left(\Delta \mathrm{G}=\Delta \mathrm{H}-\mathrm{T}^{*} \Delta \mathrm{S}\right)=-4410.09706832 E_{\mathrm{h}}$

$\begin{array}{lcccc}\text { Pd } & 1.84682384938812 & 15.08683324709847 & 29.04015349481429 \\ \text { P } & 3.38983524655574 & 16.31258254070991 & 28.09068240060263 \\ \text { N } & 0.03363674401586 & 17.84122024302360 & 28.30496716951926 \\ \text { C } & 4.07747314803517 & 17.43679668721202 & 29.41026133861902 \\ \text { H } & 4.50823441296499 & 16.69884967033780 & 30.10480486572412 \\ \text { C } & 2.93212689835179 & 18.13885259030299 & 30.15255526027506 \\ \text { H } & 2.43889700594600 & 18.83605490559117 & 29.46314170991542 \\ \text { H } & 2.18193968342447 & 17.39147021017222 & 30.43910753247445 \\ \text { C } & 3.43501361059663 & 18.89328432638823 & 31.38503770973083 \\ \text { H } & 2.59931549931442 & 19.42351772607620 & 31.85981114426611 \\ \text { H } & 3.80803620374116 & 18.16244860160949 & 32.11809464650160 \\ \text { C } & 4.55814648575012 & 19.87154780278066 & 31.03037863657535 \\ \text { H } & 4.93840634500434 & 20.35989175455699 & 31.93628619546731 \\ \text { H } & 4.15360495530738 & 20.66552609045660 & 30.38392487181467 \\ \text { C } & 5.69564083517870 & 19.15895468543711 & 30.29315578377265 \\ \text { H } & 6.47420446890827 & 19.87804610270619 & 30.00813105878652 \\ \text { H } & 6.16429392561925 & 18.42737267424738 & 30.96813829511252 \\ \text { C } & 5.18139085789811 & 18.43304402060954 & 29.04296993652501 \\ \text { H } & 6.01317433331785 & 17.92642571944167 & 28.53978904761012 \\ \text { H } & 4.77961910128933 & 19.18042130549886 & 28.34747242538143 \\ \text { C } & 4.85853638331799 & 15.34607879851124 & 27.48943564084681 \\ \text { H } & 5.65512545761514 & 16.05625842742367 & 27.23020448082240 \\ \text { C } & 5.36589417398306 & 14.42236735546014 & 28.60568493519148 \\ \text { H } & 5.69202029747524 & 15.01336591695383 & 29.46932977174720 \\ \text { H } & 4.52389538271666 & 13.80258584447442 & 28.94526891417573 \\ \text { C } & 6.50964826742007 & 13.52474248492105 & 28.12447208431713 \\ \text { H } & 7.38301974305763 & 14.14771053842582 & 27.87868661582024 \\ \text { H } & 6.81460014326602 & 12.85455708139640 & 28.93816592197946 \\ \text { C } & 6.10041692528995 & 12.71550678558396 & 26.89187741635393 \\ \text { H } & 5.28166373494552 & 12.03389042310624 & 27.16374353292652 \\ \text { H } & 6.93823610526791 & 12.09497513481901 & 26.54822937457710\end{array}$




$\begin{array}{llll}\mathrm{C} & 5.62330367165569 & 13.63739726157308 & 25.76827237750638 \\ \mathrm{H} & 5.28547435112585 & 13.04618375813558 & 24.90757692862626 \\ \mathrm{H} & 6.46549758488744 & 14.25592934238691 & 25.42373107253387 \\ \mathrm{C} & 4.48358226461184 & 14.54546068994447 & 26.23562080110937 \\ \mathrm{H} & 3.60661000425964 & 13.92505586694544 & 26.46269857275419 \\ \mathrm{H} & 4.19126531269140 & 15.22716209639368 & 25.42916026437955 \\ \mathrm{C} & 2.98007844681792 & 17.32204738889951 & 26.60128751786036 \\ \mathrm{C} & 4.00066422826579 & 18.07902978067695 & 25.99792488613863 \\ \mathrm{H} & 4.98039209922853 & 18.12114986822622 & 26.45826700246437 \\ \mathrm{C} & 3.81086709888297 & 18.74724446769345 & 24.79153046457379 \\ \mathrm{H} & 4.62572118751477 & 19.32217088072625 & 24.35806379124734 \\ \mathrm{C} & 2.58469730797605 & 18.64682970669566 & 24.13561644640163 \\ \mathrm{H} & 2.42422296781492 & 19.14445759476061 & 23.18258212444485 \\ \mathrm{C} & 1.56798158080305 & 17.88737412408311 & 24.70518590328944 \\ \mathrm{H} & 0.61116716479392 & 17.79490596714870 & 24.19653340799624 \\ \mathrm{C} & 1.73606684083506 & 17.22796916551938 & 25.93517205246261 \\ \mathrm{C} & 0.59967797856139 & 16.38349675773794 & 26.40385586916707 \\ \mathrm{C} & 0.32388039265229 & 15.22869582753952 & 25.66855412230960 \\ \mathrm{H} & 0.93748387843063 & 15.01122984377738 & 24.79788116372015 \\ \mathrm{C} & -0.67636413128896 & 14.32024141277669 & 26.05202605569014 \\ \mathrm{H} & -0.88270259172266 & 13.44211409277092 & 25.44541885309000 \\ \mathrm{C} & -1.42998385020826 & 14.60227301387682 & 27.19900055216804 \\ \mathrm{H} & -2.22179182602090 & 13.92619304262130 & 27.51368224415170 \\ \mathrm{C} & -1.19091249302879 & 15.76451786522986 & 27.92883580244661 \\ \mathrm{H} & -1.80349212718818 & 15.96304779579644 & 28.79935632819855 \\ \mathrm{C} & -0.19028022511980 & 16.68621082486552 & 27.55287645068351 \\ \mathrm{C} & -0.00429884368106 & 19.14255690006886 & 27.64275636196492 \\ \mathrm{H} & 0.44055345673529 & 19.09235922325983 & 26.65073841363760 \\ \mathrm{H} & 0.56241176038326 & 19.86726624306598 & 28.23729164544707 \\ \mathrm{H} & -1.04060872746129 & 19.50828646411469 & 27.54374564811985 \\ \mathrm{C} & -0.52051799097947 & 17.88941471854390 & 29.64800239250638 \\ \mathrm{H} & -0.28577724041886 & 16.96121411861615 & 30.17733989442528 \\ \mathrm{H} & -1.61321020566040 & 18.04772477499699 & 29.66210339867390 \\ \mathrm{H} & -0.05280291407968 & 18.72146862388439 & 30.18232069293916 \\ \mathrm{Mg} & 0.99604676101012 & 13.05181759536201 & 27.79236770959382 \\ \mathrm{Br} & 1.94176106189565 & 11.43742400994580 & 26.20456187805573 \\ \mathrm{~F} & 0.45983554006192 & 13.36218919600716 & 29.58007869697573\end{array}$

\section{0 '-MgF $2\left(\mathrm{Pd}(0)-D a v e P h o s\right.$ complex with Pd-aryl binding with $\mathrm{MgF}_{2}$ bound)}

Final Gibbs free enthalpy $\left(\Delta \mathrm{G}=\Delta \mathrm{H}-\mathrm{T}^{*} \Delta \mathrm{S}\right)=-1934.44992028 E_{\mathrm{h}}$

$\begin{array}{lccc}\text { Pd } & 2.43131636373846 & 15.04166082433687 & 28.14143734383566 \\ \mathrm{P} & 3.29191210104266 & 16.17168969945435 & 26.47944269744353 \\ \mathrm{~N} & 0.60007891712053 & 17.82326729743128 & 28.44097557196540 \\ \mathrm{C} & 4.64804483007572 & 17.23824417758735 & 27.18710413010735 \\ \mathrm{H} & 5.31707465168896 & 16.46720876931130 & 27.59847020492210\end{array}$




\begin{tabular}{|c|c|c|c|}
\hline & & & \\
\hline & & & \\
\hline & & & \\
\hline & & & \\
\hline & & & \\
\hline & 37 & & 596 \\
\hline & & & \\
\hline & & 33371 & 8509 \\
\hline & & & 104 \\
\hline & & & 005 \\
\hline & & & 923 \\
\hline & 015 & & 300 \\
\hline & & & 765 \\
\hline & & & 668 \\
\hline & & & 564 \\
\hline & & & 604 \\
\hline & 76 & & 543 \\
\hline & & & 81 \\
\hline & & & 115 \\
\hline & & & 012 \\
\hline & & & 290 \\
\hline & & & \\
\hline & & & 002 \\
\hline & 4.72 & & 310 \\
\hline & & & 220 \\
\hline & 5.20 & & 88 \\
\hline & & & 91 \\
\hline & & & 22.8 \\
\hline & & & 22. \\
\hline & & & 801 \\
\hline $\mathrm{H}$ & & & \\
\hline & & & 006 \\
\hline & 2.21 & & 400 \\
\hline & & & 101 \\
\hline & & & 881 \\
\hline & 2.03 & 18. & 462 \\
\hline & & & 6676 \\
\hline C & 0.64 & & 9383 \\
\hline & & & 5153 \\
\hline C & 0.04 & 17.9498 & 24.5 \\
\hline $\mathrm{I}$ & -1.03 & 1779111 & 52257 \\
\hline & & 17.2272 & 25.5294402 \\
\hline & 0.05045400806163 & 201274159 & 26.51446679248161 \\
\hline & & & \\
\hline & -0.52027850815053 & & 24.95247272962886 \\
\hline & -1.26297665805029 & 14.35845828915370 & 26.85642237515500 \\
\hline
\end{tabular}




$\begin{array}{llcc}\text { H } & -1.76090351659319 & 13.48662692299719 & 26.44009500018074 \\ \mathrm{C} & -1.30581799801757 & 14.62835931400662 & 28.22927720699155 \\ \mathrm{H} & -1.83161121398602 & 13.95628578370128 & 28.90395755241631 \\ \mathrm{C} & -0.70064984296685 & 15.77576347169659 & 28.74026994917605 \\ \mathrm{H} & -0.76970521563661 & 15.96811396995318 & 29.80416064044080 \\ \mathrm{C} & -0.01779996483684 & 16.68677625175162 & 27.90840959358760 \\ \mathrm{C} & 0.23220407309154 & 19.13790406322024 & 27.92616797310893 \\ \mathrm{H} & 0.05939522862888 & 19.10150707320282 & 26.85178179181051 \\ \mathrm{H} & 1.04625690475126 & 19.84524477836051 & 28.12132181351550 \\ \mathrm{H} & -0.68292745894035 & 19.51669783966149 & 28.41381010467259 \\ \mathrm{C} & 0.86523174993443 & 17.83979266451028 & 29.86957460143047 \\ \mathrm{H} & 1.32294554469964 & 16.89131891653363 & 30.16641238825502 \\ \mathrm{H} & -0.03829481447737 & 18.01292479960917 & 30.48117807910320 \\ \mathrm{H} & 1.57271277849167 & 18.64856825987100 & 30.07770695439484 \\ \mathrm{Mg} & 1.07915193434854 & 12.97724884644633 & 27.54692851300265 \\ \mathrm{~F} & 0.92253200542254 & 11.79977726017025 & 26.16492828846961 \\ \mathrm{~F} & 1.51500632662660 & 13.35470977955960 & 29.35949799050645\end{array}$

10'-PhMg( $\mu$-Br) (Pd(0)-DavePhos complex with Pd-aryl binding with PhMgBr bound and a bridging bromide)

Final Gibbs free enthalpy $\left(\Delta \mathrm{G}=\Delta \mathrm{H}-\mathrm{T}^{*} \Delta \mathrm{S}\right)=-4541.68323683 E_{\mathrm{h}}$

$\begin{array}{llcc}\text { Pd } & 1.66306542906084 & 15.18988931514617 & 29.25359490530501 \\ \text { P } & 3.23527799857074 & 16.26658627779586 & 28.09538214153582 \\ \text { N } & -0.02580303542706 & 17.94066028666647 & 28.27806446572658 \\ \text { C } & 4.04321756881458 & 17.37311519257749 & 29.36386048842585 \\ \text { H } & 4.45404541912002 & 16.61956889938046 & 30.05323472473182 \\ \text { C } & 2.96826774970636 & 18.15202708935708 & 30.13596834633285 \\ \text { H } & 2.49208576889938 & 18.87072943246300 & 29.45609260592992 \\ \text { H } & 2.18479400965125 & 17.45330306337603 & 30.45794550150804 \\ \text { C } & 3.56058503049745 & 18.88506494084761 & 31.34079099313392 \\ \text { H } & 2.77798776445506 & 19.47229521517972 & 31.83856596882372 \\ \text { H } & 3.91167592551474 & 18.13882004614789 & 32.06919717767391 \\ \text { C } & 4.72959180672014 & 19.78673211373949 & 30.93651683742458 \\ \text { H } & 5.17066099371280 & 20.25825100023851 & 31.82355834665173 \\ \text { H } & 4.35260668799123 & 20.59863558673419 & 30.29580535581432 \\ \text { C } & 5.79425974180729 & 18.99847256444416 & 30.16911405028357 \\ \text { H } & 6.60578192870326 & 19.66497560427260 & 29.84974938561479 \\ \text { H } & 6.24002144844142 & 18.24471199872617 & 30.83527489717355 \\ \text { C } & 5.19229178575049 & 18.29414012537710 & 28.94637403548396 \\ \text { H } & 5.97247440473483 & 17.72940983195732 & 28.42328705351175 \\ \text { H } & 4.81565804413890 & 19.05752188491637 & 28.25431075483413 \\ \text { C } & 4.61403128940177 & 15.17757598299678 & 27.48578336345833 \\ \text { H } & 5.46482053341051 & 15.82099462847356 & 27.22063907205435 \\ \text { C } & 5.05277384235506 & 14.20743355844977 & 28.59138709016645 \\ \text { H } & 5.41084528234958 & 14.76035528267802 & 29.46727331560553\end{array}$




\begin{tabular}{|c|c|c|c|}
\hline $\mathrm{H}$ & 4.17007807217526 & & \\
\hline $\mathrm{C}$ & & & \\
\hline & 7.05159882375170 & .81543959112096 & .86902135788496 \\
\hline & & & \\
\hline & 68381211028701 & 12.48233230172954 & \\
\hline & & & \\
\hline & & & 1873 \\
\hline & & & 785 \\
\hline & .87358634969229 & & 7024 \\
\hline & & & 683 \\
\hline & & & 361 \\
\hline & 11 & 13.8 & 552 \\
\hline & & & \\
\hline & & 17.2 & 20 \\
\hline & & & \\
\hline & & & \\
\hline & & & \\
\hline & 4.62 & & \\
\hline & 2.54 & & \\
\hline & & & \\
\hline & $1.4 \mathrm{C}$ & & \\
\hline & & & \\
\hline & & & \\
\hline & & & \\
\hline & & & \\
\hline $\mathrm{H}$ & & & \\
\hline & & & \\
\hline $\mathrm{H}$ & -1.1 & & \\
\hline & & & 27. \\
\hline & -2.4 & & \\
\hline & & & 27. \\
\hline & -1.96 & & \\
\hline & -0.3 & 577 & 036 \\
\hline & 0.00 & & \\
\hline & & & \\
\hline $\mathrm{H}$ & 0.62 & 19.9 & 28.1 \\
\hline $\mathrm{H}$ & -1.0 & & \\
\hline & -0.5 & & 29. \\
\hline & -0.3 & & \\
\hline & -1.64 & & 29.6 \\
\hline & -0.0 & & \\
\hline & & 1642885 & 78139662004 \\
\hline & & 13.57664961533856 & \\
\hline $\mathrm{C}$ & & & \\
\hline & 2.28604204221245 & 10.57545918082162 & 27.63147235162968 \\
\hline & 1.88806015061470 & 11.61215656717134 & 25.51504407922433 \\
\hline
\end{tabular}



C 3.03515684022547
9.57011977034828
27.00967644982553
H 2.17908201243367
10.53180465583566
28.71800836649541
C 2.64043130187068
10.62114730618458
24.87584100032652
H 1.46258072855078
12.40384479444371
24.89485341456244
C 3.21859579157201
9.59385771523893
25.62551112822609
H 3.48594231705830
8.77520219378679
27.60297615863565
H 2.78245829288580
10.65093442969121
23.79570249726006
H 3.81002811929558
8.82211000241266
25.13586210326915

\section{$12 F^{\prime}-M_{2 B r}\left(P d(0)-D a v e P h o s\right.$ complex with Pd-aryl binding with $\mathrm{MgBr}_{2}$ bound and 4- methoxyfluorobenzene bound)}

Final Gibbs free enthalpy $\left(\Delta \mathrm{G}=\Delta \mathrm{H}-\mathrm{T}^{*} \Delta \mathrm{S}\right)=-7331.56566323 E_{\mathrm{h}}$

$\begin{array}{llcc}\mathrm{Br} & -0.56304175295749 & -0.86131666800065 & -1.64690787964234 \\ \mathrm{C} & 0.06618080749598 & 2.69499822623168 & -1.00892757125937 \\ \mathrm{Pd} & -0.48288524915883 & 1.11013313911644 & 0.33517118979292 \\ \mathrm{Mg} & -2.08789688177515 & 1.07092842494135 & -2.03227434450812 \\ \mathrm{~F} & -0.97990756641874 & 2.86028945827330 & -2.01545087923359 \\ \mathrm{P} & -0.36349430881823 & 0.23521898544176 & 2.50669082059707 \\ \mathrm{C} & 1.00608948025174 & -1.01962207928964 & 2.58821776953621 \\ \mathrm{H} & 1.22845926792835 & -1.27048582666962 & 3.63329252971027 \\ \mathrm{C} & 2.25637297489347 & -0.38571351064671 & 1.95170339763407 \\ \mathrm{H} & 1.97895972900644 & -0.02760435387586 & 0.94889630353730 \\ \mathrm{H} & 2.57308335008306 & 0.49418955093866 & 2.52344156637331 \\ \mathrm{C} & 3.41413409075347 & -1.38062280129117 & 1.84338160181781 \\ \mathrm{H} & 4.26268982031447 & -0.90041687297957 & 1.33862427220701 \\ \mathrm{H} & 3.75637094266456 & -1.65308057201203 & 2.85264198353208 \\ \mathrm{C} & 2.99375843439333 & -2.64715036001584 & 1.09435386083255 \\ \mathrm{H} & 3.82765129794536 & -3.35951259234472 & 1.05080575356260 \\ \mathrm{H} & 2.73765412013542 & -2.38483747146276 & 0.05722394832416 \\ \mathrm{C} & 1.77454900475521 & -3.29068843644794 & 1.75919406723772 \\ \mathrm{H} & 1.45780880715119 & -4.18085754726904 & 1.20077947471787 \\ \mathrm{H} & 2.04875270751599 & -3.62917354224439 & 2.76952842338575 \\ \mathrm{C} & 0.60736153805634 & -2.30347860341250 & 1.84993401341240 \\ \mathrm{H} & -0.24262052156683 & -2.77620532581864 & 2.35348581294896 \\ \mathrm{H} & 0.28090873587569 & -2.04002352207083 & 0.83728733118545 \\ \mathrm{C} & 0.16557739341468 & 1.53105643137178 & 3.74561805553810 \\ \mathrm{H} & 1.03473003423074 & 1.97857031847420 & 3.24226102850313 \\ \mathrm{C} & -0.89092883392330 & 2.63375251009796 & 3.88401020896558 \\ \mathrm{H} & -1.17158664329333 & 3.00462139482785 & 2.89463344407501 \\ \mathrm{H} & -1.79416072440264 & 2.20512638003543 & 4.33987102476262 \\ \mathrm{C} & -0.39123326208591 & 3.78997963640503 & 4.75556426762274 \\ \mathrm{H} & 0.44947136633701 & 4.28487645990610 & 4.24682695655867 \\ \mathrm{H} & -1.18423842114933 & 4.54100106174821 & 4.86372049776606 \\ \mathrm{C} & 0.07390004862415 & 3.30048245587305 & 6.12851989072093 \\ \mathrm{H} & -0.79006220061145 & 2.90187026125458 & 6.68066866834816\end{array}$




\begin{tabular}{|c|c|c|c|}
\hline & & & \\
\hline & 1.12636697385873 & & \\
\hline & 1.42309730357061 & 1.82240199572905 & 97361122006320 \\
\hline & & & \\
\hline & 60992398791491 & 1.03815490237705 & 833311 \\
\hline & & 0.57371046938574 & 5.63763542398241 \\
\hline & 1.39008837028706 & 0.27534688329411 & 5.03501559231330 \\
\hline & -1.82305699869685 & & 2074 \\
\hline & -1.68443131525497 & 496 & 5704 \\
\hline & -0.70941216160030 & & 606 \\
\hline & -2.76608023743770 & -2.0979974 & 3850 \\
\hline & 13299089 & -2.7 & 7664 \\
\hline & -4.021 & & 827 \\
\hline & 54303 & -2.5 & 9577 \\
\hline & -4.173 & & \\
\hline & 1211 & -1 & 810 \\
\hline & -3.09 & & 800 \\
\hline & -3.372 & & 493 \\
\hline & & & \\
\hline & -3.072 & & 642 \\
\hline & -3.819 & & \\
\hline & -3.83 & & \\
\hline & $-4.2 \varepsilon$ & & 717 \\
\hline & -4.2 & & 396 \\
\hline & $-4.6^{\prime}$ & & 397 \\
\hline & -3.81 & & 057 \\
\hline & -0.12 & & \\
\hline & & & \\
\hline & 3.43 & & \\
\hline & -1.08 & & 226 \\
\hline & 2.45 & & -0.7 \\
\hline & 1.027 & & \\
\hline & 0.92 & & 36 \\
\hline & 1.35 & & -1.5 \\
\hline & 2.293 & & 097 \\
\hline & 5950703886 & 9528068 & 3314 \\
\hline & & & \\
\hline J & 7105276 & & 52632 \\
\hline & -2.927 & & \\
\hline & -2.44174270060547 & 2548242 & 3013782257 \\
\hline & -2.15127176780124 & & \\
\hline 1 & -3.43282637132667 & -3.26561071424429 & 1.20761936253610 \\
\hline & -4.57845425546079 & & \\
\hline & -4.76838650030485 & & -1.04890987700090 \\
\hline & -5.54147900848868 & -1.62994574272819 & -1.34079901308849 \\
\hline & -3.98967614046318 & -2.02485812904790 & -2.13791566049843 \\
\hline
\end{tabular}




$\begin{array}{llll}\mathrm{O} & 3.33796287225031 & 3.64620701282574 & 1.36482393259340 \\ \mathrm{C} & 4.65012148508129 & 3.22182495483188 & 0.98565315582995 \\ \mathrm{H} & 5.30625649391315 & 3.52744044665282 & 1.80413169531936 \\ \mathrm{H} & 4.97552913681683 & 3.70556437887541 & 0.05520833167497 \\ \mathrm{H} & 4.69238426814925 & 2.13031805328503 & 0.86663264473112\end{array}$

12F'-Mg( $\mu$-Br)F (Pd(0)-DavePhos complex with Pd-aryl binding with MgBrF bound with a bridging bromide and 4-methoxyfluorobenzene bound)

Final Gibbs free enthalpy $\left(\Delta \mathrm{G}=\Delta \mathrm{H}-\mathrm{T}^{*} \Delta \mathrm{S}\right)=-4855.91912428 E_{\mathrm{h}}$

$\begin{array}{llcc}\mathrm{Br} & -0.56811108809223 & -0.85674493529394 & -1.65739813631796 \\ \mathrm{C} & 0.07641868721730 & 2.69332652860068 & -1.01368031214848 \\ \mathrm{Pd} & -0.48212801219835 & 1.10705240793379 & 0.33173373347618 \\ \mathrm{Mg} & -2.09541571635327 & 1.07868790315334 & -2.06304978863623 \\ \mathrm{~F} & -0.95735457543425 & 2.85810795703276 & -2.02584891982321 \\ \mathrm{P} & -0.36584971420707 & 0.23523461169555 & 2.50178723251125 \\ \mathrm{C} & 1.00333896551974 & -1.02041790322092 & 2.58576760497486 \\ \mathrm{H} & 1.22033784232320 & -1.27609442503098 & 3.63079412998716 \\ \mathrm{C} & 2.25727998665930 & -0.38439951556074 & 1.95873247483165 \\ \mathrm{H} & 1.98477516789853 & -0.02008473204590 & 0.95681275901573 \\ \mathrm{H} & 2.57267356846427 & 0.49197701758566 & 2.53684451750714 \\ \mathrm{C} & 3.41474960863163 & -1.37969600699669 & 1.84986337515452 \\ \mathrm{H} & 4.26586199205735 & -0.89725190868904 & 1.35165653382970 \\ \mathrm{H} & 3.75227194195830 & -1.65834219035441 & 2.85906876990215 \\ \mathrm{C} & 2.99684855667995 & -2.64165328520693 & 1.09187695700912 \\ \mathrm{H} & 3.83043374036779 & -3.35441831787116 & 1.04792301451412 \\ \mathrm{H} & 2.74536831131590 & -2.37343083438098 & 0.05512699767706 \\ \mathrm{C} & 1.77436302563473 & -3.28785883916917 & 1.74795564568982 \\ \mathrm{H} & 1.45924577418413 & -4.17473428732057 & 1.18332353877751 \\ \mathrm{H} & 2.04417369875927 & -3.63219001826877 & 2.75754282768215 \\ \mathrm{C} & 0.60752861129569 & -2.30021395377005 & 1.83905779739991 \\ \mathrm{H} & -0.24474997125061 & -2.77506208668158 & 2.33670894986220 \\ \mathrm{H} & 0.28541743001743 & -2.03112675273683 & 0.82652491008454 \\ \mathrm{C} & 0.16473549754567 & 1.53164936014638 & 3.74006995560893 \\ \mathrm{H} & 1.03255998060690 & 1.97967771599288 & 3.23509567392265 \\ \mathrm{C} & -0.89219276800717 & 2.63377937669404 & 3.87956907168228 \\ \mathrm{H} & -1.17381081565480 & 3.00396331801226 & 2.89020119002304 \\ \mathrm{H} & -1.79485459849044 & 2.20478972220690 & 4.33617802152026 \\ \mathrm{C} & -0.39253469242948 & 3.79046710531051 & 4.75056234845078 \\ \mathrm{H} & 0.44727138318985 & 4.28603698676316 & 4.24101380398714 \\ \mathrm{H} & -1.18607054185341 & 4.54082519845300 & 4.85969822076410 \\ \mathrm{C} & 0.07480291579895 & 3.30149907178451 & 6.12300700602695 \\ \mathrm{H} & -0.78798811400360 & 2.90179160089912 & 6.67624700728492 \\ \mathrm{H} & 0.47380699608574 & 4.13864618286010 & 6.71003846063683 \\ \mathrm{C} & 1.12833377817986 & 2.20107246161837 & 5.97960534544721 \\ \mathrm{H} & 1.42726103903241 & 1.82516111894389 & 6.96615132776660\end{array}$




\begin{tabular}{|c|c|c|c|}
\hline & 2.03071031656847 & & \\
\hline & & & \\
\hline & -0.23985554564291 & 0.57432882111569 & 5.63311858774806 \\
\hline & 1.39278670648585 & & 5.02752736759016 \\
\hline & -1.82446569878920 & -0.59575811883923 & 3.25010188846383 \\
\hline & -1.68313121919586 & -1.40944885 & 3282 \\
\hline & -0.70713090908840 & & \\
\hline & -2.76318073034268 & -2.09 & \\
\hline & -2.62377315628569 & -2.71 & \\
\hline & -4.01978521241945 & 908996 & 0027 \\
\hline & -4.87 & -2.51 & \\
\hline & 020466436 & -1.21 & 0914 \\
\hline & -5.1 & & \\
\hline & -3.094 & -0.51 & 4551 \\
\hline & -3.37774153917589 & 0.3118173 & \\
\hline & -3.38 & & \\
\hline & -3.07 & 2.10 & \\
\hline & -3.82 & $2.5^{\prime}$ & \\
\hline & -3.8 & 3.64 & 366 \\
\hline & -4.28 & & \\
\hline & -4.2 & 0.6 & 1651 \\
\hline & -4.6 & & \\
\hline & -3.819 & -0.26 & \\
\hline & -0.120 & 3.34 & 07 \\
\hline & 1.48 & & \\
\hline & 3.44 & 2.409 & -1.0 \\
\hline I & $-1.0^{\prime}$ & 57 & 530 \\
\hline & 2.46 & 2.67 & 427 \\
\hline & 1.02 & 3.67 & 780 \\
\hline & 0.92 & 4.22 & 2901 \\
\hline C & & & \\
\hline C & 2.294 & 3.323 & \\
\hline F & -2.866 & 1.31 & \\
\hline $\mathrm{H}$ & $-4.6^{\prime}$ & & 5375 \\
\hline & $-3.8^{\prime}$ & -1.61 & \\
\hline & -2.921 & -2.55 & 7597 \\
\hline $\mathrm{H}$ & -2.432 & -3.10 & \\
\hline 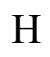 & -2.147 & -2.0297002 & 36057 \\
\hline $\mathrm{H}$ & -3.41 & -3.269177 & \\
\hline $\mathrm{C}$ & & & \\
\hline $\mathrm{H}$ & -4.778 & +2963189034 & 826143 \\
\hline $\mathrm{H}$ & -5.552 & -1.63 & -1.32506984560081 \\
\hline I & -4.00451717959599 & -2.03051662721357 & -2.12864382823883 \\
\hline $\mathrm{O}$ & 3.3339 & & \\
\hline & 4.64709218774017 & 3.20416110643392 & 1.01913491328984 \\
\hline & 5.29858791766540 & 3.51021922242851 & 1.84114593917382 \\
\hline
\end{tabular}



H 4.98083444438207
3.68319612039798
0.08936118221566
H $4.68710895889488 \quad 2.11230452712038 \quad 0.90407441695333$ 


\section{References}

(1) Noël-Duchesneau, L.; Lugan, N.; Lavigne, G.; Labande, A.; César, V. Customized Buchwald-Type Phosphines Bearing an "Inverted" Pyrimidinium Betaine as an Aryl Group Surrogate - Synthesis and Coordination Chemistry with Gold(I). Eur. J. Inorg. Chem. 2015, 1752-1758.

(2) Cho, C.-H.; Park, H.; Park, M.-A.; Ryoo, T.-Y.; Lee, Y.-S.; Park, K. Solid-Phase Synthesis of Biphenyls and Terphenyls by the Traceless Multifunctional Cleavage of Polymer-Bound Arenesulfonates. Eur. J. Org. Chem. 2005, 3177.

(3) Nguyen, M. H.; Smith, A. B. Recyclable Polystyrene-Supported Siloxane-Transfer Agent for Palladium-Catalyzed Cross-Coupling Reactions. Org. Lett. 2014, 16, 2070.

(4) Yuen, O. Y.; So, C. M.; Man, H. W.; Kwong, F. Y. A General Palladium-Catalyzed Hiyama Cross-Coupling Reaction of Aryl and Heteroaryl Chlorides. Chem. Eur. J. 2016, 22, 6471.

(5) Iglesias, M. J.; Prieto, A.; Nicasio, M. C. Kumada-Tamao-Corriu Coupling of Heteroaromatic Chlorides and Aryl Ethers Catalyzed by (IPr)Ni(allyl)Cl. Org. Lett. 2012, 14, 4318 .

(6) Shi, S.; Meng, G.; Szostak, M. Synthesis of Biaryls through Nickel-Catalyzed SuzukiMiyaura Coupling of Amides by Carbon-Nitrogen Bond Cleavage. Angew. Chem. Int. Ed. 2016, 55, 6959.

(7) Denmark, S. E.; Smith, R. C.; Chang, W.-T. T.; Muhuhi, J. M. Cross-Coupling Reactions of Aromatic and Heteroaromatic Silanolates with Aromatic and Heteroaromatic Halides. J. Am. Chem. Soc. 2009, 131, 3104.

(8) Shen, A.; Ni, C.; Cao, Y.-C.; Zhou, H.; Song, G.-H.; Ye, X.-F. Novel monoligated imine-Pd-NHC complexes: extremely active pre-catalysts for Suzuki-Miyaura coupling of aryl chlorides. Tetrahedron Lett. 2014, 55, 3278.

(9) Li, X.-J.; Zhang, J.-L.; Geng, Y.; Jin, Z. Nickel-Catalyzed Suzuki-Miyaura Coupling of Heteroaryl Ethers with Arylboronic Acids. J. Org. Chem. 2013, 78, 5078.

(10) Izawa, Y.; Zheng, C.; Stahl, S. S. Aerobic Oxidative Heck/Dehydrogenation Reactions of Cyclohexenones: Efficient Access to meta-Substituted Phenols. Angew. Chem. Int. Ed. 2013, 52, 3672.

(11) Hirai, Y.; Uozumi, Y. Preparation of Aryl(dicyclohexyl)phosphines by C-P BondForming Cross-Coupling in Water Catalyzed by an Amphiphilic-Resin-Supported Palladium Complex. Synlett 2017, 28, 2966.

(12) Neese, F. The ORCA program system. WIREs Comput. Mol. Sci. 2012, 2, 73.

(13) (a) Schaefer, A.; Horn, H.; Ahlrichs, R. Fully optimized contracted Gaussian basis sets for atoms Li to Kr. J. Chem. Phys. 1992, 97, 2571; (b) Schaefer, A.; Huber, C.; Ahlrichs, R. Fully optimized contracted Gaussian basis sets of triple zeta valence quality for atoms Li to Kr. J. Chem. Phys. 1994, 100, 5829; (c) Weigend, F.; Ahlrichs, $\mathrm{R}$. Balanced basis sets of split valence, triple zeta valence and quadruple zeta valence quality for H to Rn: Design and assessment of accuracy. Phys. Chem. Chem. Phys. 2005, 7, 3297; (d) Weigend, F. Accurate Coulomb-fitting basis sets for H to Rn. Phys. Chem. Chem. Phys. 2006, 8, 1057. 